\title{
Ultraschallspektrometrie zum dynamischen Verhalten von Domänen in peptidhaltigen Lipidmembranen
}

\author{
Dissertation \\ zur Erlangung des Doktorgrades \\ der Mathematisch-Naturwissenschaftlichen Fakultäten \\ der Georg-August-Universität Göttingen
}

vorgelegt von

Markus Jäger

aus

Holzminden

Göttingen 2005 
Referent:

Korreferent:

Tag der mündlichen Prüfung:
Prof. Dr. D. Ronneberger Prof. Dr. K. Bärner

4. Juli 2005 


\section{Inhaltsverzeichnis}

$\begin{array}{ll}\text { 1. Einleitung } & 1\end{array}$

2. Biophysikalische Grundlagen 3

2.1. Biologische Membranen . . . . . . . . . . . . . . . . . . . . 3

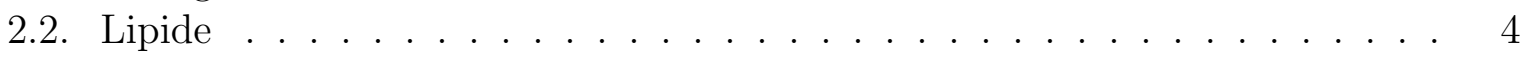

2.2.1. Membranlipide ................... . . 4

2.2.2. DMPC ............................ 4

2.2.3. Der Phasenübergang . . . . . . . . . . . . . . . . . . 6

2.3. Das Peptid Alamethicin . . . . . . . . . . . . . . . . 8

3. Grundlagen der Ultraschallabsorptionsspektrometrie 13

3.1. Die klassische Dämpfung . . . . . . . . . . . . . . . . . . . . . . . . . . . . 13

3.2. Einzelzeitrelaxation . . . . . . . . . . . . . . . . . . . . 14

3.3. Die Theorie von Bhattacharjee und Ferrell . . . . . . . . . . . . . . . 16

4. Messverfahren der Ultraschallabsorptionsspektrometrie $\quad 21$

4.1. Der piezoelektrische Wandler . . . . . . . . . . . . . . . . . . . . . 21

4.2. Das Resonatorverfahren . . . . . . . . . . . . . . . . . . . . . . . . . . . . . . . . . . 23

4.2.1. Der ideale Resonator . . . . . . . . . . . . . . . . . . 23

4.2.2. Der reale Resonator . . . . . . . . . . . . . . . . . . . . . 25

4.2.3. Einfluss der Wandlereigenschaften . . . . . . . . . . . . . . . . . 27

4.2.4. Aufbau des Messplatzes . . . . . . . . . . . . . . . . . . . 29

4.2.5. Die verwendeten Resonatoren . . . . . . . . . . . . . . . . . 30

4.2.6. Ablauf der Messung . . . . . . . . . . . . . . . . . . 33

4.2.7. Der Messplatz zur Messung der erweiterten

Spektralfunktion $\mathrm{S}^{\#} \ldots \ldots \ldots \ldots \ldots$. . . . . . . . . . 34

4.2.8. Das Programm NWAControl . . . . . . . . . . . . . . . . 36

4.2.9. Fehlerabschätzung . . . . . . . . . . . . . . . . . . . . 40

4.3. Das Schwingungspulstransmissionsverfahren . . . . . . . . . . . . . . 42

4.3.1. Das Messprinzip . . . . . . . . . . . . . . . . . . . 42

4.3.2. Der Messplatz für das Schwingungspulstransmissionsverfahren . . . 43

4.3.3. Ablauf der Messung . . . . . . . . . . . . . . . . . . . 44

4.3.4. Fehlerabschätzung für das Schwingungspulstransmissionsverfahren . 50

4.4. Die Schallgeschwindigkeitsmessung . . . . . . . . . . . . . . 51

4.4.1. Grundlagen der Schallgeschwindigkeitsmessung . . . . . . . . . 51

4.4.2. Das kommerzielle Spektrometer HR-US 102 . . . . . . . . . . . . 54

4.4.3. Aufbau des Velocimetrie-Messplatzes . . . . . . . . . . . . . 55 
4.4.4. Auswertung der Schallgeschwindigkeitsdaten . . . . . . . . . . . 56

4.4.5. Fehlerabschätzung . . . . . . . . . . . . 57

4.5. Densitometrie . . . . . . . . . . . . . . . . . 58

4.5.1. Geräteeigenschaften und Messprinzip . . . . . . . . . . . . 58

4.5.2. Ablauf der Messung . . . . . . . . . . . . . . . . 59

4.5.3. Messfehler . . . . . . . . . . . . . . . . . . 59

5. Probenpräparation $\quad \mathbf{6 1}$

5.1. Das Ansetzen der Lipidsuspension . . . . . . . . . . . . . . . . . . . 61

5.2. Präparation der Vesikel . . . . . . . . . . . . . . . . . . . . . . 64

6. Messergebnisse der Ultraschallspektrometrie $\quad 65$

6.1. Die Spektren . . . . . . . . . . . . . . . . . . . . 65

6.1.1. Reines DMPC . . . . . . . . . . . . . . . 65

6.1.2. Messergebnisse der DMPC/Alamethicin-Suspensionen . . . . . . . . 70

6.2. Interpretation der Messergebnisse . . . . . . . . . . . . . . 76

6.2.1. Die empirische Skalierungsfunktion . . . . . . . . . . . . . . 76

6.2.2. Die axiale Diffusion . . . . . . . . . . . . . . . . . . . . . . . . . . . . . . . 84

6.2.3. Die Vesikeldeformation . . . . . . . . . . . . . . . . . . 87

7. Messergebnisse aus Densitometrie und Velocimetrie $\quad 91$

7.1. Temperaturabhängige Untersuchungen von Dichte und Schallgeschwindigkeit 91

7.2. Untersuchung der Schallgeschwindigkeit in Abhängigkeit von der Alamethicinkonzentration . . . . . . . . . . . . . . . . 95

$\begin{array}{ll}\text { 8. Zusammenfassung und Ausblick } & 99\end{array}$

A. Darstellung der Ultraschallspektren I

A.1. DMPC:Alamethicin, 99,7:0,3 mol\% . . . . . . . . . . . . . I I

A.2. DMPC:Alamethicin, 99,5:0,5 mol\% . . . . . . . . . . . . IV

A.3. DMPC:Alamethicin, 99,3:0,7 mol\% . . . . . . . . . . . . VI

A.4. DMPC:Alamethicin, $99: 1 \mathrm{~mol} \% \ldots \ldots \ldots$. . . . . . . . . . .

B. Bildschirmfotos von NWAControl XIII

$\begin{array}{ll}\text { C. Velocimetrie-Skript } & \text { XVII }\end{array}$

Literaturverzeichnis $\quad$ XIX

$\begin{array}{ll}\text { Danksagung } & \text { XXV }\end{array}$

$\begin{array}{lll}\text { Lebenslauf } & \text { XXVII }\end{array}$ 


\section{Einleitung}

Die Entstehung der biologischen Membran war grundlegend für die Evolution des Lebens auf der Erde. Sie führte zu der Bildung erster geschlossener Bioreaktoren [77], in denen auf engstem Raum chemische Reaktionen ablaufen konnten. Im Laufe des vergangenen Jahrhunderts wurde mit Hilfe der Mikroskopie und kontinuierlich verbesserten biophysikalischen Verfahren die Struktur der biologischen Membran immer besser bekannt.

Das Fluid-Mosaic-Modell von Singer und Nicolson [102], das im Jahr 1972 veröffentlicht wurde, ist als Meilenstein in der Membranforschung anzusehen. Es war bereits bekannt, dass die Membran aus einer Doppelschicht aus Phospholipiden geformt wird. Phospholipide besitzen eine hydrophile Kopfgruppe und hydrophobe Alkylketen. Singer und Nicolson postulieren in ihrem Modell die freie Beweglichkeit der Moleküle in der Membranebene. Wie in einem Mosaik sind funktionelle Gruppen aus Transmembranproteinen, peripheren Proteinen und Glycoproteinen in der Membran verteilt, die sich ihrerseits auch frei in der Membran bewegen können. Sie dienen zum Beispiel dem gerichteten Transport von Ionen oder der interzellulären Erkennung.

In den vergangenen Jahrzehnten wurden große Fortschritte in der Strukturbestimmung und der Analyse der Stoffwechselvorgänge in Membranen erzielt. Bei diesen Untersuchungen wurde das Augenmerk insbesondere auf die Proteine gelegt. Seit einigen Jahren rückt nun auch das Wechselspiel von Proteinen und Lipiden in das Zentrum des Interesses.

Biologische Membranen bestehen aus bis zu einhundert verschiedenen Lipidtypen. Die Zusammensetzung der Membran entscheidet über die makroskopischen Eigenschaften der Fluidität und der „Schmelztemperatur“. Lipide mit gleichen physikalischen Eigenschaften organisieren sich in Domänen, die unter anderem speziellen Proteinen die Einlagerung in die Membran ermöglichen.

Eine effektive Methode zur Untersuchung von Membranvorgängen ist die Breitbandultraschallabsorptionsspektrometrie, oder kürzer: Ultraschallspektrometrie. Mit ihr lassen sich unter anderem chemische Prozesse detektieren, die mit einer Volumenänderung einhergehen, wie z. B. Konformationsänderungen oder die Komplexierung von Molekülen. Dabei wird die Kopplung periodischer Druckschwankungen an die Volumenänderung des chemischen Prozesses ausgenutzt. Nach dem Prinzip von LeChatelier folgt das System der Druckänderung durch Verschiebung des Reaktionsgleichgewichts [86]. Aus der charakteristischen Zeitspanne dieser Antwort, der so genannten Relaxationszeit, kann auf molekulare Wechselwirkungen im Zusammenhang mit diesem Prozess geschlossen werden.

Das Verfahren ist allerdings sensitiv gegenüber allen Prozessen, die mit einer Volumenänderung einhergehen. Das Gesamtspektrum wird daher durch eine Summe unterschiedlicher Prozesse beschrieben. Wie im Laufe dieser Arbeit deutlich wird, ist die Interpretation eines solchen Spektrums bereits für einfache Systeme sehr aufwändig. Die Analyse einer natürlichen biologischen Membran mit all ihren unterschiedlichen Komponenten mit Hilfe der Ultraschallspektrometrie ist zur Zeit nicht eindeutig durchführbar. 
Als Alternative zur Untersuchung der Membraneigenschaften bieten sich hier einfache Modellmembranen an. Hierbei werden Vesikel - oder Liposome - einer einzigen Lipidsorte betrachtet. Eines der meist untersuchten Lipide ist 1,2-Dimyristoyl-sn-Glycerin-3-Phosphatidylcholin (DMPC). In zahlreichen Arbeiten wurde das Verhalten von DMPC-Membranen [40], cholesterolhaltiger DMPC-Membranen [98] und binärer Lipidmembranen [20, 43, 98] untersucht.

In dieser Arbeit wird ein erster Schritt zur Klärung der Kinetik in peptidhaltigen Lipidmembranen gemacht. Dabei liegt der Schwerpunkt auf der Untersuchung des dynamischen Verhaltens der Domänen. In DMPC-Membranen bilden sich Domänen aus Lipiden in unterschiedlichen Phasen: den gelförmigen Lipiden mit geordneten Alkylketten und den fluiden Lipiden, deren Alkylketten ungeordnet vorliegen. Die Domänen sind ständigen Fluktuationen unterworfen: Lipide an den Domänengrenzen wechseln kontinuierlich zwischen dem gelförmigen und fluiden Zustand und führen somit zu makroskopischen Fluktuationen der Membrandichte.

Der Übergang von der gelförmigen Phase in die fluide Phase wird auch als „Schmelzen“ der Membran bezeichnet. Das Schmelzen ist ein kooperativer Prozess, der in einem sehr engen Temperaturbereich stattfindet. In diesem Bereich sind auch die Dichtefluktuationen besonders stark, so dass der Einfluss von Peptiden hier besonders deutlich zu sehen ist.

Der Einfluss der Dichtefluktuationen in binären Flüssigkeiten in der Nähe des kritischen Entmischungspunktes auf das Ultraschallabsorptionsspektrum wird durch die dynamische Skalierungstheorie von Bhattacharjee und Ferrell [15, 17] beschrieben, die eine Relaxationszeitverteilung für die Fluktuationen herleiten. Halstenberg et al. [42] zeigen, dass diese Theorie auch auf Membransysteme angewandt werden kann. Aufgrund einer Verlangsamung aller Prozesse in der Nähe der Umwandlungstemperatur, dem Critical Slowing Down, korrespondieren die Relaxationszeiten von DMPC- und DMPC/Cholesterolmembranen mit Frequenzen im niederfrequenten Bereich von $100 \mathrm{kHz}$ bis $10 \mathrm{MHz}$.

Dieser Messbereich wird in der Ultraschallspektrometrie durch das so genannte Resonatorverfahren abgedeckt. Nahe der Umwandlungstemperatur ändert sich die Ultraschallabsorption stark bei Variation der Temperatur, daher ist in besonderem Maße auf die Temperaturstabilität während der Messung zu achten. Ein weiterer Aspekt ist der Anstieg der Absorption zu der Umwandlungstemperatur hin. Der Messaufbau muss in der Lage sein, über einen großen Absorptionsbereich gut zu funktionieren. Um diesen Anforderungen gerecht $\mathrm{zu}$ werden, wurde ein neuer Messplatz erstellt, der durch den Einsatz zweier kleinvolumiger Resonatoren den gewünschten Frequenzbereich abdeckt und eine hohe Temperaturstabilität aufweist. Gesteuert wird der Messplatz durch das Programm NWAControl, das durch einen adaptiven Scan-Algorithmus in der Lage ist, sowohl geringe Dämpfungen fern der Umwandlungstemperatur als auch hohe Dämpfungen in der Nähe der Umwandlung zu registrieren.

Auf der Basis von NWAControl wird auch eine weitere Anwendung des Resonatorverfahrens ermöglicht: die temperaturabhängige Velocimetrie [75]. Diese erlaubt zusammen mit der Densitometrie Aussagen über die Kompressibilität der Membranen zu machen.

Am Ende dieser Arbeit werden einige neue Erkenntnisse über die Wechselwirkungen von Peptiden und Lipiden in biologischen Membranen stehen, aber es werden auch Probleme diskutiert, die in kommenden Untersuchungen zu lösen sind. 


\section{Biophysikalische Grundlagen}

\subsection{Biologische Membranen}

Biologische Membranen spielen in der gesamten Biosphäre eine entscheidende Rolle. Sie legen eine Außengrenze von Zellen fest und regeln den Austausch von Molekülen über diese Grenze. Sie unterteilen den Innenraum in diskrete Kompartimente, wodurch Prozesse und Zellbestandteile voneinander getrennt werden.

Membranen sind widerstandsfähig, aber flexibel, gestatten Formveränderungen und sind selektiv permeabel für gelöste Stoffe. Durch die Fähigkeit, Brüche in der Oberfläche selbst wieder verschließen zu können, ermöglichen sie auch die Verschmelzung zweier Membranen oder die Spaltung eines abgeschlossenen Kompartiments.

Membranen enthalten zudem eine Vielzahl von Proteinen, die auf die Katalyse zahlreicher molekularer Prozesse spezialisiert sind. Pumpen transportieren z. B. gelöste spezifische Stoffe gegen einen Konzentrationensgradienten durch die Membran; Rezeptoren auf der Membran nehmen extrazelluläre Signale wahr und setzen sie in molekulare Veränderungen in der Zelle um.

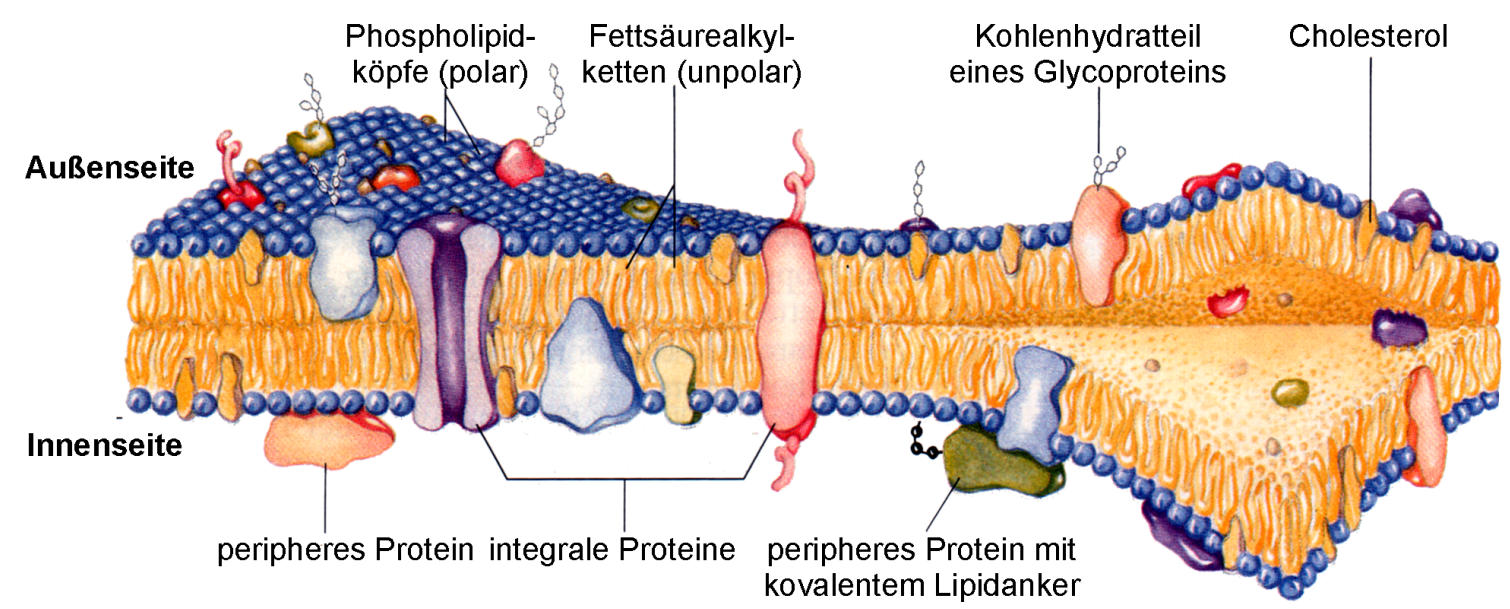

Abbildung 2.1: Das Fluid-Mosaic-Modell nach Singer und Nicolson [102]. Die Abbildung [77] zeigt die wesentlichen Bestandteile einer biologischen Membran. Zu erkennen ist die Doppelschicht bestehend aus Phospolipiden, in der Lipide und Proteine in der lateralen Ebene frei diffundieren können. Cholesterol ist allerdings nach aktuellem Wissensstand nicht in den Kopfgruppen der Lipide eingebettet, wie hier dargestellt, sondern in der Region der hydrophoben Ketten.

Membranen bestehen nur aus zwei Molekülschichten (Bilayer) und sind damit sehr dünn. Man kann sie daher praktisch als zweidimensional annehmen. Intermolekulare Prozesse 
sind an einer zweidimensionalen Oberfläche sehr viel wahrscheinlicher als im dreidimensionalen Raum, deshalb ist die Effizienz bestimmter Stoffwechselaktivitäten innerhalb einer zweidimensionalen Membran stark erhöht [77].

Die Struktur der Membran wird durch das Fluid-Mosaic-Modell von Singer und Nicolson [102] beschrieben. Wie in Abb. 2.1 dargestellt, wird die Membran durch eine Doppelschicht aus Phospholipiden (z. B. DMPC) und Steroiden (z. B. Cholesterol) gebildet.

In diese Doppelschicht sind in unregelmäßigen Abständen Proteine eingebettet, die durch hydrophobe Wechselwirkungen zwischen Membranlipiden und hydrophoben Domänen in den Proteinen festgehalten werden. Die Proteine sind asymmetrisch in der Membran angeordnet, wodurch die Membran „verschiedenseitig“ wird. Die Transmembranproteine gehen durch die gesamte Membran und können durch Komplexierung Kanäle oder Ionenpumpen bilden.

Die einzelnen Lipide und Proteinuntereinheiten bilden ein so genanntes „flüssiges Mosa$\mathrm{ik}^{*}$, das seine Gestalt ständig verändert. Das Membranmosaik ist flüssig, weil die LipidLipid-Bindungen und die Lipid-Peptid-Bindungen nicht kovalent sind. Einzelne Lipid- und Proteinmoleküle können sich daher frei in der Membranebene bewegen.

\subsection{Lipide}

Lipide umfassen eine Vielzahl unterschiedlicher Moleküle, deren Gemeinsamkeit die Unlösbarkeit in Wasser ist. Gerade dies führt bei zahlreichen Glycerophospholipiden zur Ausbildung von Membranen. Andere Lipide, wie z.B. die Triacylglyceride dienen der Energiespeicherung [77].

\subsubsection{Membranlipide}

Membranlipide sind amphipatische Moleküle mit einer polaren hydrophilen Kopfgruppe und einer unpolaren hydrophoben Kettenregion. Man kann zwischen drei allgemeinen Typen von Membranlipiden unterscheiden: den Glycerophospholipiden, wie DMPC, das als Ausgangslipid für die verwendeten Modellmembranen dient, ferner den Steroiden, wie Cholesterin, die stabilisierend in der Membran wirken, und den Sphingolipiden, die an unterschiedlichen interzellulären Erkennungsprozessen beteiligt sind [77].

In natürlichen biologischen Membranen kommen bis zu einhundert verschiedene Lipidtypen vor. Um Aussagen über die physikalischen Eigenschaften treffen zu können, ist eine Reduktion der Lipidanzahl erforderlich. In dieser Arbeit wird eine Modellmembran mit einem einzigen Lipidtyp betrachtet.

\subsubsection{DMPC}

Für die Untersuchungen wird ein Modellsystem basierend auf dem Glycerophospholipid 1,2-Dimyristoyl-sn-Glycerin-3-Phosphatidylcholin, kurz DMPC, verwendet. 

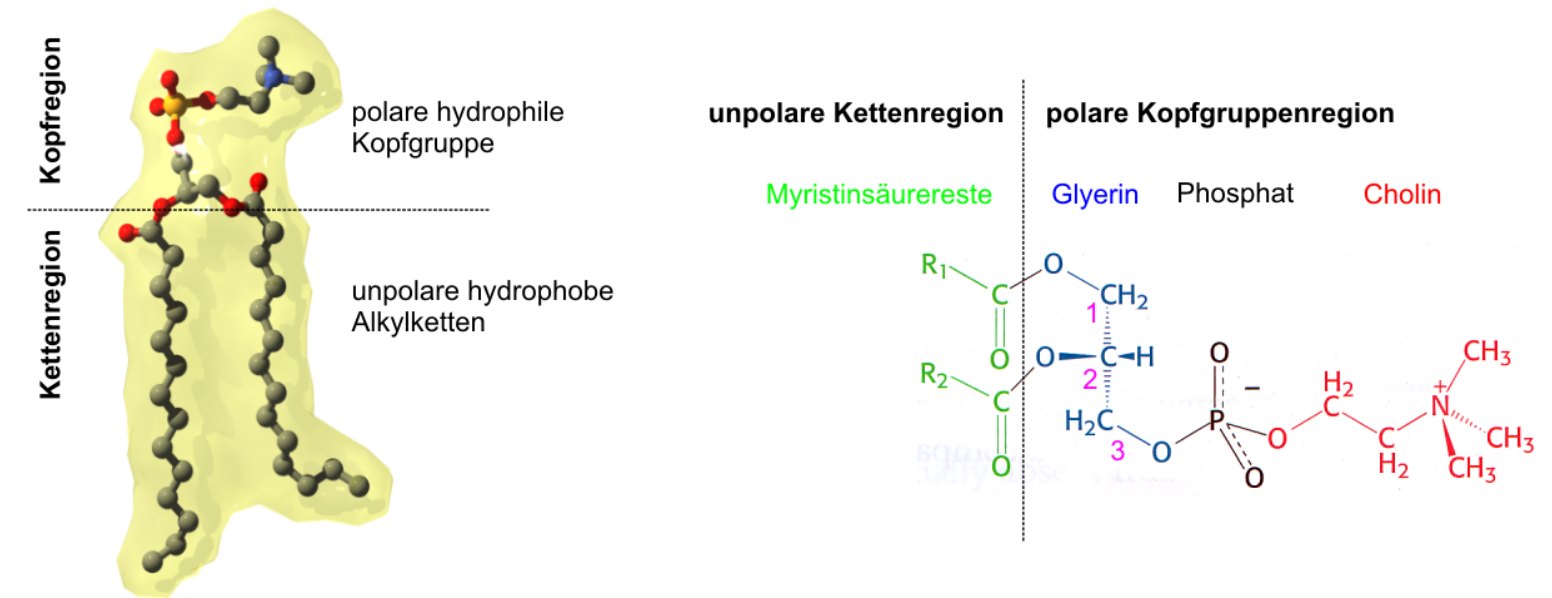

\begin{abstract}
Abbildung 2.2: Schematische Darstellung von 1,2-Dimyristoyl-sn-Glycerin-3-Phosphatidylcholin. Links ist die molekulare Struktur von DMPC als Ball-and-Stick-Modell dargestellt. Die errechnete Moleküloberfläche ist als gelbliche Tönung hinzugefügt worden [11, 38]. Es kann eine Teilung in eine polare hydrophile Kopfgruppenregion und eine unpolare hydrophobe Kettenregion angenommen werden. Die Kettenregion besteht bei DMPC aus zwei Myristinsäureresten, die an den C1- und C2-Atomen des Glycerins gebunden sind. In der rechten Hälfte der Grafik ist die Strukturformel der Kopfgruppenregion dargestellt, die aus einem Glycerin, der Phosphatgruppe und einem Alkoholrest, dem Cholin besteht [13].
\end{abstract}

DMPC ist ein typisches Membranlipid, das in zahlreichen biologischen Membranen zu finden ist. In Glycerophospholipiden sind zwei Fettsäuren über eine Esterbrücke mit dem C1- und dem C2-Atom des Glycerins verknüpft, wie in Abb. 2.2 dargestellt. Am C3-Atom ist ein polarer Alkohol über eine Phosphodiesterverbindung gebunden. Alle Glycerophospholipide tragen bei pH 7 eine negative Ladung an der Phosphatgruppe. Der Kopfgruppenalkohol kann bei einem pH-Wert um 7 ebenfalls eine oder mehrere Ladungen tragen [77].

DMPC trägt als Fettsäurereste am C1- und C2-Atom des Glycerins jeweils eine Myristinsäure, bestehend aus einer gesättigten Kohlenwasserstoffkette mit 14 C-Atomen. Als Alkoholrest ist eine Cholingruppe mit dem Phosphat verestert.

Für Untersuchungen mit Hilfe der Ultraschallspektrometrie im Bereich des Phasenübergangs von Membranen, wie im nächsten Abschnitt beschrieben, sind aus technischen Gründen Übergangstemperaturen von $T=5-50^{\circ} \mathrm{C}$ wünschenswert. Die Übergangstemperatur von DMPC beträgt $T_{\mathrm{m}}=24,0^{\circ} \mathrm{C}$ [37].

Löst man Lipide in Wasser, aggregieren sie spontan in verschiedenen Konfigurationen, wie Mizellen, Vesikeln oder in der hexagonal kubischen Konfiguration. Die Aggregation hängt insbesondere von den geometrischen Eigenschaften des Lipids, d. h. dem Verhältnis der Größe von Kopf- und Kettenregion ab.

DMPC bildet in wässriger Umgebung multilamellare Vesikel aus, die aus einer Vielzahl von Lipiddoppelschichten bestehen. Die laterale Ordnung der Lipide in den Lipidschichten hängt entscheidend von der Temperatur ab. Für DMPC kann man im Temperaturbereich zwischen $5{ }^{\circ} \mathrm{C}$ und $50^{\circ} \mathrm{C}$ drei verschiedene Phasen unterscheiden, deren geometrische Anordnung in Abb. 2.3 dargestellt ist. 

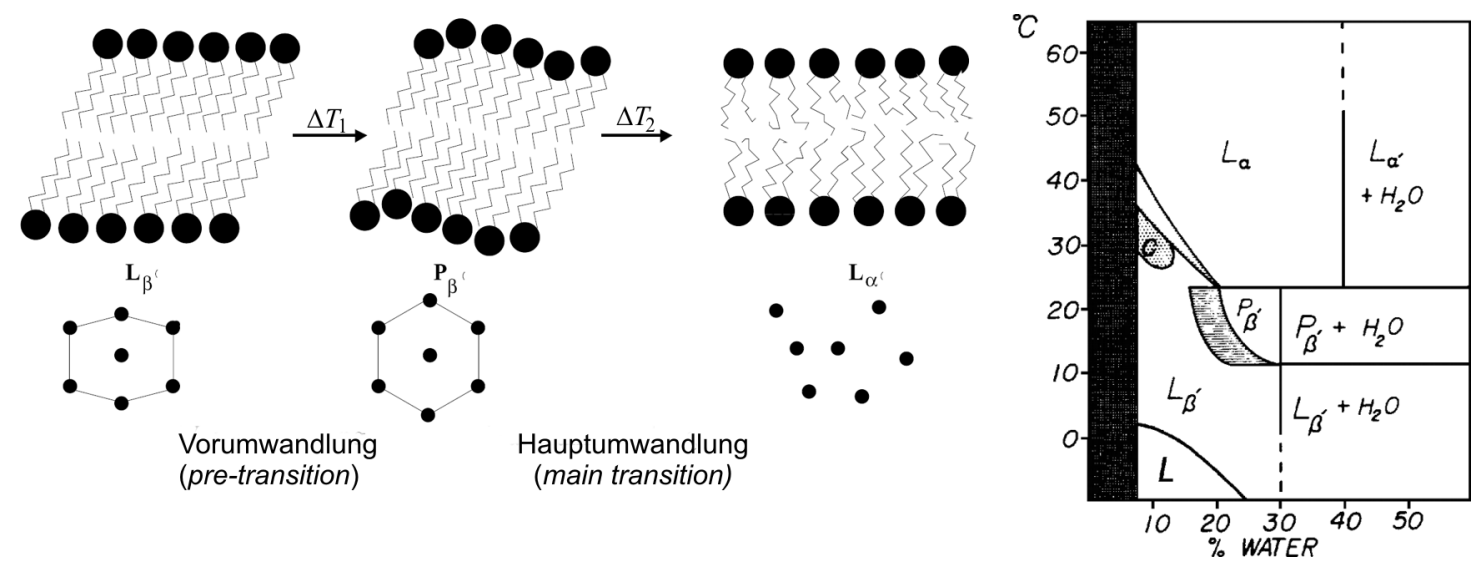

Abbildung 2.3: Phasendiagramm von DMPC. Auf der linken Seite dieser Abbildung ist die geometrische Anordnung der DMPC-Phasen dargestellt. In der $P_{\beta^{\prime}}$-Phase nimmt die Membranoberfläche eine wellenförmige Gestalt an, die man als Ripple-Phase bezeichnet. Die hexagonale Anordnung der $L_{\beta^{\prime}}$-Gelphase bleibt hierbei erhalten. In der fluiden $L_{\alpha^{\prime}}$ Phase geht die laterale Ordnung verloren. Die rechte Grafik zeigt ein Phasendiagramm von DMPC/Wasser-Suspensionen. Die untersuchten Proben weisen einen Wassergehalt $>99 \%$ auf. Die entsprechenden Phasen sind am rechten Rand des Diagramms zu finden [60].

Der Übergang von der gelförmigen Phase $\left(L_{\beta^{\prime}}\right)$ in die Ripple-Phase $\left(P_{\beta^{\prime}}\right)$ wird als Vorumwandlung (pre-transition) bezeichnet und ist bei $T_{\mathrm{pre}} \approx 14{ }^{\circ} \mathrm{C} \mathrm{zu}$ finden. Die spezifische Dichte- und Volumenänderung ist bei diesem Übergang mit den zur Verfügung stehenden spektroskopischen Methoden nicht nachweisbar. Der Vorgang kann jedoch mit anderen Methoden wie der Kalorimetrie untersucht werden [50].

Sowohl Gelphase als auch Ripple-Phase zeigen eine hohe laterale Ordnung. Aus diesem Grunde werden diese Phasen häufig auch als Gelphase zusammengefasst. Mit zunehmender Temperatur verschwindet die laterale Ordnung und die Lipide gehen in die flüssigkristalline oder fluide Phase $\left(L_{\alpha^{\prime}}\right)$ über. Die Hauptumwandlung (main transition) liegt für DMPC bei $T_{\mathrm{m}}=24,0^{\circ} \mathrm{C}$ [37]. Bei dieser Temperatur liegen Moleküle in gelförmigem und fluidem Zustand in gleichen Teilen in der Membran vor. Aufgrund der Volumenänderung während dieses Übergangs ist es möglich, den Phasenübergang mit Hilfe der Ultraschallspektrometrie zu untersuchen, wie in Abschnitt 3 beschrieben wird.

\subsubsection{Der Phasenübergang}

Phasenübergänge lassen sich durch die Einführung eines Ordnungsparameters $\phi$ charakterisieren. Aufgrund der Dichteänderung beim Übergang von der gelförmigen in die fluide Phase in DMPC-Membranen ist es sinnvoll, die Dichte als Ordnungsparameter anzunehmen. Anhand der Stetigkeit des Ordnungsparameters können Phasenübergänge erster und zweiter Ordnung unterschieden werden. Bei einem Phasenübergang erster Ordnung ändert sich der Ordnungsparameter sprunghaft am Phasenübergang. Phasenübergänge zweiter Ordnung sind durch einen stetigen Verlauf des Ordnungsparameters gekennzeichnet.

Verschiedene kalorimetrische Messungen und Monte-Carlo-Simulationen zeigen, dass es sich bei der Hauptumwandlung in Lipiden um einen Phasenübergang zweiter Ordnung 
handelt $[47,48,62]$.

Die charakteristischen Eigenschaften des Phasenübergangs 2. Ordnung können zusammengefasst werden [88, 104]:

- Es wird keine latente Umwandlungswärme für den Phasenübergang benötigt.

- Es tritt keine Koexistenz beider Phasen während des Phasenübergangs auf.

- Die Entropie $S(T, p)$ und das Volumen $V(T, p)$ sind am Umwandlungspunkt stetig.

- Die isobare Wärmekapazität $C_{\mathrm{p}}$, sowie die isothermen und isobaren Kompressibilitäten $\kappa_{\mathrm{T}}$ und $\kappa_{\mathrm{p}}$ zeigen am Phasenübergang ein unstetiges Verhalten.

Während eines Phasenübergangs zweiter Ordnung treten starke Fluktuationen der Ordnungsparameter in der Nähe der Umwandlungstemperatur auf. Dieses Verhalten unterscheidet sich grundlegend von dem Verhalten weit entfernt vom Phasenübergang und wird als kritisches Verhalten bezeichnet.

Die Ginzburg-Landau-Theorie [88] gibt eine phänomenologische Erklärung für dieses Verhalten. Das Verschwinden des Ordnungsparameters $\phi$ am Phasenübergangsgangs führt zu der Annahme, dass die freie Energie $\mathcal{F}$ in der Nähe des Phasenübergangs um $\phi=0$ wie folgt entwickelt werden kann:

$$
\mathcal{F}=\mathcal{F}_{0}+\int d^{n} r\left\{a_{2} \phi^{2}+a_{4} \phi^{4}+g(\nabla \phi)^{2}\right\}
$$

mit

$$
\begin{gathered}
\mathcal{F}_{0}: \text { freie Energie für } \phi=0 \\
a_{0}, a_{2}, a_{4}, g: \text { Entwicklungskoeffizienten. }
\end{gathered}
$$

Die Definitionen eines Ordnungsparameter $\phi=\rho_{\text {gel }}-\rho_{\mathrm{fl}}$ und $\phi=\rho_{\mathrm{fl}}-\rho_{\text {gel }}$ sind äquivalent, so dass in Gl. (2.1) aufgrund der Symmetrie der Hauptumwandlung nur gerade Potenzen von $\phi$ auftreten. Betrachtet man den Phasenübergang in Abhängigkeit von der Temperatur, so erhält man

$$
a_{2} \sim\left|\frac{T-T_{\mathrm{c}}}{T_{\mathrm{c}}}\right|,
$$

wobei $T_{\mathrm{c}}$ die kritische Temperatur bzw. Übergangstemperatur ist.

Für einen Phasenübergang zweiter Ordnung lassen sich mit Hilfe dieser Proportionalität weiterhin die folgenden Eigenschaften angeben:

- Die Korrelationslänge $\xi$ divergiert nach einem Potenzgesetz der Form $\xi \sim\left|\left(T-T_{\mathrm{c}}\right) / T_{\mathrm{c}}\right|^{-\tilde{\nu}}$ mit dem kritischen Exponent der Korrelationslänge $\tilde{\nu}$. In der Nähe der kritischen Temperatur wird das System daher mangels einer ausgezeichneten Längenskala skaleninvariant. 
- Alle thermodynamischen Ordnungsparameter folgen der Skaleninvarianz ebenfalls einem Potenzgesetz in der Form $\phi \sim\left|\left(T-T_{\mathrm{c}}\right) / T_{\mathrm{c}}\right|^{\gamma}$ nahe der kritischen Temperatur.

- Die Dynamik des Systems wird für $T \rightarrow T_{\mathrm{c}}$ sehr langsam. Dieser Effekt wird als Critical Slowing Down bezeichnet. Die dynamische Erweiterung der Landau-Theorie liefert unter Vernachlässigung der nichtlinearen Anteile [55]

$$
\partial_{t} \phi(x, t)=\Gamma \nabla^{2} \frac{\delta \mathcal{F}}{\delta \phi} \approx 2 \Gamma a_{2} \nabla^{2} \phi(x, t) .
$$

Durch eine Fourier-Transformation ergibt sich die Lösung dieser Gleichung

$$
\phi(x, t) \sim e^{-t / \tau} \quad \text { mit } \quad \tau=\frac{1}{2 \Gamma a_{2} \beta^{2}},
$$

wobei

$$
\begin{aligned}
& a_{2}: \text { Entwicklungskoeffizient } \\
& \Gamma: \text { kinetischer Exponent } \\
& \beta \text { : Wellenzahl. }
\end{aligned}
$$

Die Lösung dieser Gleichung zeigt, dass die charakteristische Zeitskala $\tau$ für $a_{2} \rightarrow 0$, d. h. für Temperaturen in der Nähe des kritischen Punktes divergiert. Die Dynamik des Systems wird dadurch dramatisch verlangsamt.

- Die isobare Wärmekapazität $C_{\mathrm{p}}$ und die isotherme Kompressibilität $\kappa_{\mathrm{T}}$ divergieren an der kritischen Temperatur ebenfalls nach einem Potenzgesetz. Das Verhalten lässt sich anhand des Dissipations-Fluktuationsgesetzes bestimmen [49]:

$$
\begin{aligned}
& C_{\mathrm{p}}=\left(\frac{d\langle H\rangle}{d T}\right)_{\mathrm{p}}=\frac{\left\langle H^{2}\right\rangle-\langle H\rangle^{2}}{R T^{2}} \\
& \kappa_{\mathrm{T}}=-\frac{1}{\langle V\rangle}\left(\frac{d\langle V\rangle}{d p}\right)_{\mathrm{T}}=\frac{\left\langle V^{2}\right\rangle-\langle V\rangle^{2}}{\langle V\rangle \cdot R T^{2}}
\end{aligned}
$$

mit

$C_{\mathrm{p}}, \kappa_{\mathrm{T}}$ : isobare Wärmekapazität und isotherme Kompressibilität

$H, V$ : Exzessenthalpie und Volumen

$p, T$ : Druck und absolute Temperatur

$R$ : universelle Gaskonstante.

\subsection{Das Peptid Alamethicin}

Proteine sind die häufigsten und vielfältigsten Makromoleküle in lebenden Zellen. Sie bestehen aus Ketten, die aus bis zu zwanzig verschiedenen Aminosäuren gebildet werden und über kovalente Bindungen verknüpft sind. Ihre Diversität ist durch die Reihenfolge 
der Aminosäuren (Primärstruktur) gewährleistet. Kleine Proteine, deren Molekulargewicht unter $10^{4}$ Dalton liegt, werden im Allgemeinen als Peptide bezeichnet. Proteine und Peptide können über Wasserstoffbrückenbindungen Sekundärstrukturen wie die $\alpha$-Helix oder die $\beta$-Faltblattstruktur ausbilden. Durch weitere Molekülfaltung und Komplexierung können größere Struktureinheiten, die Tertiär- und Quartärstrukturen, entstehen.

Die Aminosäuren werden anhand ihrer Seitenketten in fünf Hauptklassen eingeteilt: (a) unpolar und aliphatisch, (b) aromatisch (gewöhnlich unpolar), (c) polar, aber ungeladen, (d) negativ geladen und (e) positiv geladen. Innerhalb jeder Klasse gibt es Abstufungen in der Polarität, Größe und Form der Seitenketten [77].

Diese Eigenschaften der Aminosäuren haben einen großen Einfluss auf die Faltung und die Funktion der Proteine. In wässriger Umgebung kann z. B. der hydrophobe Effekt zu einer Faltung der Peptidkette führen, wenn es für das Protein energetisch günstigere Konformationen gibt, in der unpolare Aminosäuren vom Wasser abgeschirmt sind. Ein ähnliches Verhalten kann man bei dem Peptid Alamethicin finden. Allerdings ist das Molekül zu klein, um eine effektive Abschirmung durch Faltung zu erreichen. Es bettet sich stattdessen in die hydrophoben Kettenregionen einer Membrandoppelschicht ein.

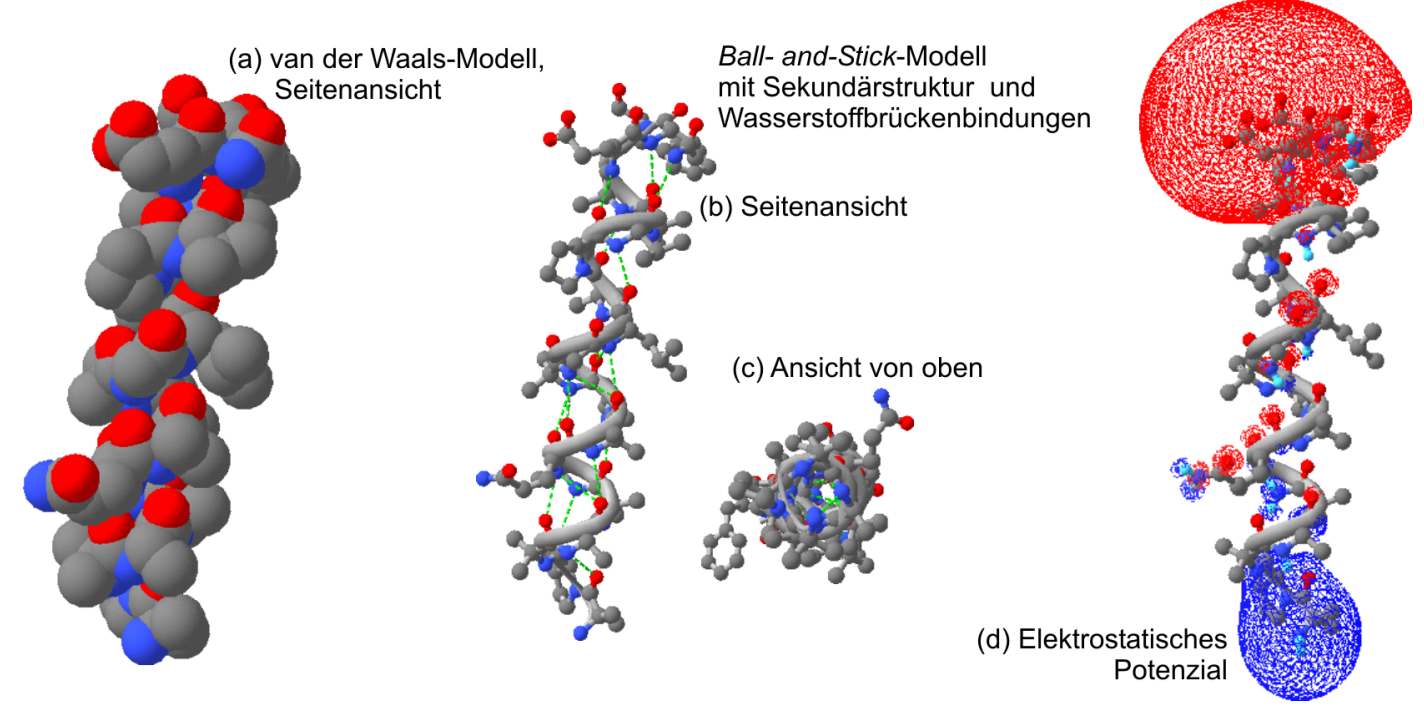

Abbildung 2.4: Darstellung der Struktur von Alamethicin. In (a) ist das Modell von Alamethicin anhand der kristallographischen Daten von Fox und Richards [11, 35] dargestellt. In (b) und (c) wird die helikale Struktur betont. Für (d) wurde zusätzlich das elektrostatische Potenzial berechnet [38]. Man erkennt den polaren Charakter an den Endgruppen.

Alamethicin, das aus dem Pilz Trichoderma viride gewonnen wird, besteht aus zwanzig Aminosäuren [27]. Es bildet bevorzugt eine helikale Struktur aus. Wie Abb. 2.4 zeigt, besitzt das Molekül größtenteils einen hydrophoben unpolaren Charakter, lediglich die Aminosäuren an C- und N-Terminus weisen einen polaren Charakter auf. Die Größe des Moleküls, die etwa der Dicke einer Lipiddoppelschicht entspricht, und die Ladungsverteilung im Molekül erlauben die Einbettung in die Membran. Dabei lagern sich die Endgruppen des Alamethicins in die polaren Kopfgruppen der Lipide ein, während der hydrophobe Bereich zwischen den Lipidalkylketten eingebettet wird. Der Mechanismus, der zur Integration von Alamethicin führt, wird an späterer Stelle mit Hilfe der Messdaten ausführlich diskutiert. 


\section{Porenbildung durch $\alpha$-helikale Peptide}

Alamethicin gehört zu der Gruppe der $\alpha$-helikalen Peptiden, die eine breite antimikrobiale Wirkung durch die Lysis, also die Zerstörung, von Membranen zeigen [12, 28, 110]. Die Lysis wird durch die Bildung von Poren hervorgerufen [57]. Obwohl die Bildung von Poren schon seit über dreißig Jahren bekannt ist [5], ist die Physik dieses Prozesses noch nicht vollständig verstanden.

Die Bildung von Poren in Lipidmembranen ist immer mit dem Aufbau einer Spannung verbunden. Die Energie, die zur Bildung nötig ist, hängt insbesondere von der mechanischen Membranspannung und der Energie ab, die zur Ausbildung des Porenrandes notwendig ist $[57,112]$. Die Porenbildung ist ein statistischer Effekt und findet häufig an Nukleationsdefekten statt.

Bevor es zur Ausbildung von Poren kommt, werden die $\alpha$-helikalen Peptide in der Kopfgruppenregion der Lipidmembran adsorbiert [29, 46, 112]. Molekulardynamik(MD)-Simulationen zeigen, dass sich die $\alpha$-Helix des Alamethicin in der Nähe der Kopfgruppen von 1,2-Dioleoyl-sn-Glycerin-3-Phosphatidylcholin (DOPC) stabilisiert [108].

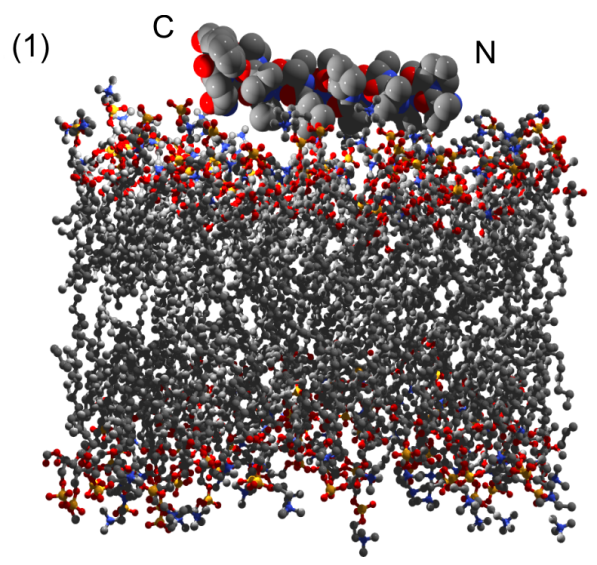

(2a)

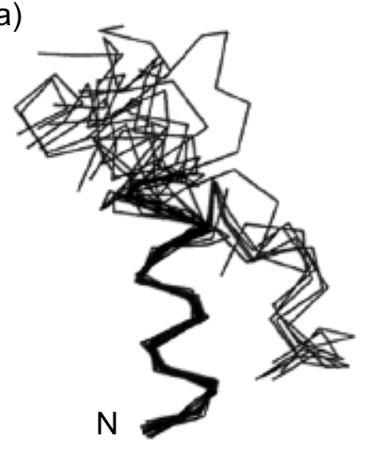

(2b)

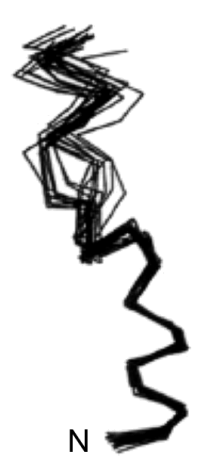

Abbildung 2.5: Adsorption von Alamethicin auf der Membranoberfläche. (1) Veranschaulichung der Adsorption von Alamethicin auf einer DMPC-Membran [11, 38]. Berechnung der Position der Aminosäuren mittels MD-Simulation: (2a) in freiem Wasser, (2b) im Hydratwasser der Lipidmembran [108].

Die Simulationen zeigen, dass Alamethicin Bindungen zu den polaren Kopfgruppen der Lipide ausbildet, was zu einer Stabilisierung führt [112]. Ein großer Beitrag zur Stabilisierung kann auch der Hydrathülle der Membran zugeschrieben werden. In der Hülle ist die Diffusion der Wassermoleküle gegenüber dem freien (bulk) Wasser deutlich verringert $\left(D_{\text {hyd }} \approx 0,2 \cdot D_{\text {bulk }}\right)$. Es treten auch gerichtete Verbindungen zwischen Wasser und Lipid auf. Die Stabilisierung des Alamethicins wird daher wahrscheinlich zusätzlich durch zwei Faktoren begünstigt [108]:

- Die Ausbildung von Wasser/Lipid-Bindungen ist gegenüber Wasser/AlamethicinBindungen energetisch günstiger.

- Die Entfaltung des Peptids wird verlangsamt, weil die Diffusion des Wassers herabgesetzt ist. 
Die Anlagerung an der Oberfläche führt zu einer lateralen Verschiebung der Lipide und damit zu einer lokalen Deformation der Membran [46, 51]. Es kann gezeigt werden, dass diese lokalen Deformationen, die einen Durchmesser von ungefähr $40 \AA$ besitzen, die Adsorption an der Oberfläche begrenzen. Ab einem kritischen Peptid/Lipid-Verhältnis überlappen sich die lokalen Deformationen der einzelnen Peptide. Eine weitere Adsorption ist energetisch ungünstig, so dass von nun an die Peptide in die Membran integriert werden oder eine Translokation auf die andere Seite der Membran stattfindet [57]. Die kritische Konzentration variiert über einen weiten Bereich und ist abhängig von Lipid- und Peptidtyp. Untersuchungen hierzu wurden an z. B. an Alamethicin/DPhPC (1,2-Diphytanoylsn-Glycerin-3-Phosphatidylcholin, [58]) und Melittin/DOPC-Membranen bei $30{ }^{\circ} \mathrm{C}$ in der Gelphase durchgeführt [29]. Die Deformationen begünstigen das Schmelzen der Membran und führen so zu einer lokalen Verringerung der Membrandicke um ungefähr 5\%. Einer Theorie zufolge ist die Deformationsenergie die treibende Kraft der Peptidintegration [46].

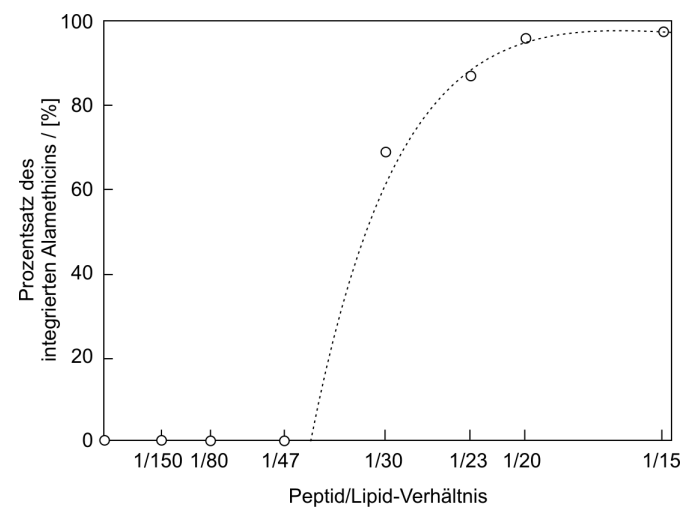

Abbildung 2.6: Anteil des integrierten Alamethicins. Die Grafik zeigt den Anteil integrierter Alamethicin-Moleküle in einer DPhPC-Membran in Abhängigkeit der Peptid/LipidKonzentration. Bis zu einer Konzentration von $1 / 47$, entsprechend 2 mol\% werden die Peptide lediglich auf der Oberfläche adsorbiert. Bei $5 \mathrm{~mol} \%$ sind bereits fast alle Moleküle in die Membran integriert [46].

Alamethicin bildet bei der Integration Poren aus 6 - 11 Peptidmolekülen [37, 44, 45]. Während Alamethicin Poren nach dem Barrel-Stave-Modell ausbildet, können die Poren eines anderen gut untersuchten Peptids, des Melittins, durch das toroidale Modell beschrieben werden. Beide Modelle sind in Abb. 2.7 dargestellt.

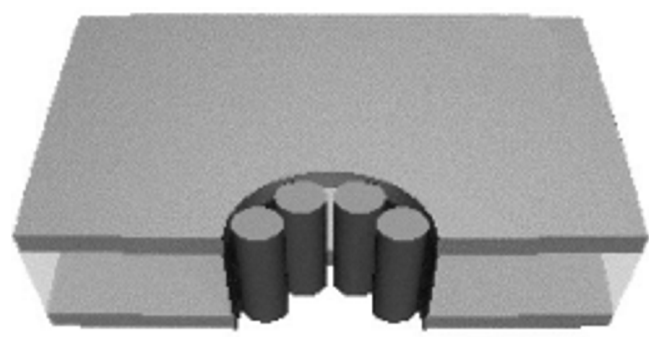

Barrel-Stave-Modell

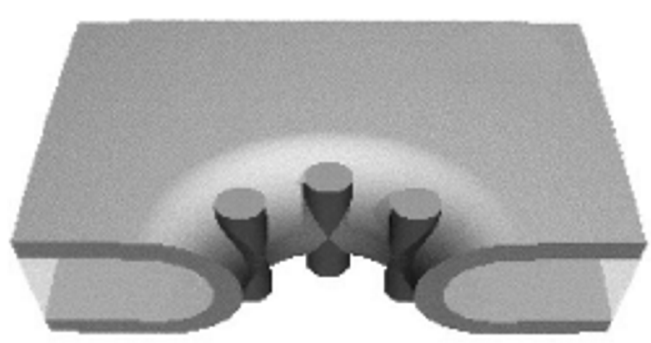

Toroidales Modell

Abbildung 2.7: Modelle zur Organisation von Poren. $\alpha$-helikale Peptide bilden unterschiedliche Poren aus. Während Alamethicin Barrel-Stave-Poren ausbildet, die durch das Peptid begrenzt werden, wird bei vielen anderen Peptiden die Membran in diesem Bereich derart gekrümmt, dass die Pore durch Lipide begrenzt wird [109]. 


\section{Grundlagen der Ultraschallabsorptionsspektrometrie}

Die Ultraschallabsorptionsspektrometrie erlaubt, schnelle molekulare Prozesse in einem fluiden Medium nachzuweisen. Grundlage dieses Verfahrens ist die Bestimmung des frequenzabhängigen Absorptionskoeffizienten $\alpha(\omega)$, der in der exponentiellen Abklingfunktion einer ebenen, harmonischen Schallwelle zum Ausdruck kommt [83]:

$$
p(x, t)=p_{0} e^{i \omega t} e^{-\gamma x} \quad \text { mit } \gamma=\alpha+i \beta,
$$

wobei

$p_{0}$ : Schalldruckamplitude zur Zeit $t=0$ am Ursprungsort $x=0$

$\omega=2 \pi f:$ Kreisfrequenz der Druckwelle

$\gamma$ : komplexer Ausbreitungskoeffizient

$\alpha:$ Absorptionskoeffient

$\beta=2 \pi / \lambda$ : Wellenzahl $\beta$ mit der Wellenlänge $\lambda$.

\subsection{Die klassische Dämpfung}

Die klassische Dämpfung kann im Rahmen der Kontinuumsmechanik berechnet werden. Die Rechnung baut dabei auf der Kontinuitätsgleichung

$$
\frac{\partial}{\partial t} \rho(\vec{x}, t)+\rho \vec{\nabla} \cdot \vec{u}(\vec{x}, t)=0
$$

mit der Dichteverteilung $\rho(\vec{x}, t)$ und dem Geschwindigkeitsfeld $\vec{u}(\vec{x}, t)$ und der NavierStokes-Gleichung in der Komponentendarstellung

$$
\partial_{t}\left(\rho u_{i}\right)+\partial_{j}\left(\rho u_{i} u_{j}\right)=-\partial_{i} p+\partial_{j} \sigma_{i j} \quad i, j=1,2,3
$$

mit dem Spannungstensor $\sigma_{i j}$ auf. Für einfache Flüssigkeiten kann diese Gleichung vereinfacht werden und in der Vektornotation angegeben werden als

$$
\rho \frac{\partial \vec{u}}{\partial t}=-\vec{\nabla} \vec{p}+\left(\frac{4}{3} \eta_{\mathrm{s}}+\eta_{\mathrm{v}}\right) \vec{\nabla}^{2} \vec{u}
$$

mit

$\vec{p}(\vec{x}, t)$ : Druckfeld

$\eta_{\mathrm{s}}, \eta_{\mathrm{v}}:$ Scher- und Volumenviskosität. 
Die Viskositäten treten als Elemente des Spannungstensors aus Gl. (3.3) auf, der sich aus der Überlegung ergibt, dass sich eine propagierende Welle durch eine Überlagerung einer Scherung und einer Kompression darstellen lässt [10].

Der Dämpfungsexponent $\alpha(\omega)$ ergibt sich aus der Lösung der Gln. (3.2) und (3.3) zu

$$
\begin{aligned}
\alpha(\omega) & =\frac{\omega^{2}}{2 c_{\mathrm{s}}^{3} \rho}\left(\frac{4}{3} \eta_{\mathrm{s}}+\eta_{\mathrm{v}}\right) \\
\Rightarrow \alpha(f) & =\frac{2 \pi^{2}}{c_{\mathrm{s}}^{3} \rho}\left(\frac{4}{3} \eta_{\mathrm{s}}+\eta_{\mathrm{v}}\right) \cdot f^{2}
\end{aligned}
$$

Die Schallgeschwindigkeit $c_{\mathrm{s}}$ ist dabei über die Newton-Laplace-Gleichung unter der Vernachlässigung der Wärmeleitfähigkeit mit der adiabatischen Kompressibilität $\kappa_{\mathrm{s}}$ verknüpft:

$$
c_{\mathrm{s}}=\sqrt{\frac{1}{\kappa_{\mathrm{s}} \rho}} .
$$

Die Wärmeleitung liefert einen weiteren Beitrag zur Hintergrunddämpfung, der allerdings häufig vernachlässigt werden kann.

Die Hintergrunddämpfung, oder klassische Dämpfung genannt, zeigt eine Proportionalität

$$
\alpha \sim f^{2} \Rightarrow\left(\frac{\alpha}{f^{2}}\right)=\text { const. }
$$

Die daher naheliegende $\left(\alpha / f^{2}\right)$-Darstellung der Spektren ermöglicht es, zusätzliche Absorptionsbeiträge $\left(\alpha / f^{2}\right)_{\mathrm{ex}}$, auch als Exzessdämpfung $(\alpha \lambda)_{\mathrm{ex}}$ bezeichnet, leicht vom konstanten Hintergrund zu unterscheiden:

$$
\left(\frac{\alpha}{f^{2}}\right)_{\mathrm{ges}}=\left(\frac{\alpha}{f^{2}}\right)_{\mathrm{ex}}+\left(\frac{\alpha}{f^{2}}\right)_{\mathrm{kl}} .
$$

Häufig wird die Dämpfung als dimensionslose Absorption entlang einer Wellenlänge $(\alpha \lambda)$ dargestellt. In der Ultraschallspektrometrie werden häufig die klassische Dämpfung $(\alpha \lambda)_{\mathrm{kl}}$ und Exzessbeiträge hochfrequenter Relaxationsprozesse $(\alpha \lambda)_{\mathrm{ex}}^{\mathrm{hf}}$, die oberhalb des gemessenen Spektrums liegen und nicht näher charakterisiert werden können, durch den so genannten $B$-Wert ausgedrückt

$$
\begin{aligned}
(\alpha \lambda)_{\mathrm{kl}}+(\alpha \lambda)_{\mathrm{ex}}^{\mathrm{hf}} & =B \cdot f \\
\Rightarrow(\alpha \lambda)_{\mathrm{ges}} & =(\alpha \lambda)_{\mathrm{ex}}+B \cdot f .
\end{aligned}
$$

\subsection{Einzelzeitrelaxation}

Der Gleichgewichtszustand eines geschlossenen thermodynamischen Systems hängt von seinen Zustandsgrößen, wie Temperatur, Druck und Konzentrationen ab. Läuft im geschlossenen System eine chemische Reaktion

$$
\mathrm{A}+\mathrm{B} \underset{k_{\mathrm{b}}}{\stackrel{k_{\mathrm{f}}}{\rightleftharpoons}} \mathrm{AB} \quad \text { oder } \quad \mathrm{A} \underset{k_{\mathrm{b}}}{\stackrel{k_{\mathrm{f}}}{\rightleftharpoons}} \mathrm{A}^{*},
$$


mit den Reaktionsraten $k_{\mathrm{f}}$ und $k_{\mathrm{b}} \mathrm{ab}$, dann lässt sich das Reaktionsgleichgewicht durch Variation der äußeren Parameter verschieben. Im folgenden sei $\mathrm{A} \rightleftharpoons \mathrm{A}^{*}$ eine Konformationsänderung, wobei für die Volumina gelte $V_{\mathrm{A}}>V_{\mathrm{A}^{*}}$. Erhöht man den äußeren Druck auf das System, so wird das Gleichgewicht nach dem Prinzip von LeChatelier [86] zu Gunsten der Bildung von A* verlagert. Bei instantanen Druckänderungen, wie in Drucksprungexperimenten, benötigt das System eine Relaxationszeit $\tau$ mit

$$
\tau^{-1}=k_{\mathrm{f}}^{-1}+k_{\mathrm{b}}^{-1}
$$

um in den neuen Gleichgewichtszustand zu relaxieren. Die dazugehörige Konzentrationsänderung lässt sich durch ein Exponentialgesetz in der Form

$$
-\frac{d c_{\mathrm{A}}}{d t}=-\frac{1}{\tau}\left(c_{\mathrm{A}}-c_{\mathrm{A}^{*}}\right)
$$

beschreiben, wobei $c_{\mathrm{A}}$ die Konzentration des Eduktes ist und $c_{\mathrm{A}^{*}}$ die Konzentration des Produktes im neuen Gleichgewicht darstellt.

Instantane Druckänderungen sind in realen Experimenten nur bis zu einer Auflösung von Zeiten $\tau \geq 1 \mu$ s durchführbar. Eine Druckänderung benötigt eine endliche Zeit, die eine Auflösungsgrenze für schnelle Prozesse darstellt.

Um schnellere molekulare Prozesse untersuchen zu können, kann ein zeitlich veränderliches harmonisches Druckfeld angelegt werden. In diesem Fall ist eine Dissipation der eingestrahlten Energie zu beobachten. Bei nicht verschwindendem Reaktionsvolumen sowie einer Phasenverschiebung zwischen der zeitlich veränderlichen Druckwelle $p(t)$ und zeitlich variierender Volumenänderung $V(t)$ erhält man einen nichtverschwindenden dissipativen Beitrag

$$
W=-\int_{0}^{2 \pi / \omega} p(t) d V(t)
$$

Das Maximum dieser Funktion liegt bei $\omega \tau=1$, wobei $\tau$ die Relaxationszeit des chemischen Prozesses bezeichnet. Für $\omega \tau \ll 1$ kann das System entsprechend der Druckänderung in den bevorzugten Zustand ohne nennenswerte Phasenverschiebung relaxieren; für $\omega \tau \gg 1$ ist es dem System nicht mehr möglich, während einer Druckperiode zu relaxieren.

Im Absorptionsspektrum erhält man aufgrund dieser Dissipation einen Beitrag zur Exzessdämpfung in der Form [105]

$$
\alpha(\omega)_{\mathrm{ex}}=\frac{c_{\mathrm{s}} \rho}{2 R T} G\left(\Delta V-\frac{a_{\mathrm{p}}}{\rho C_{\mathrm{p}}} \Delta H\right)^{2} \frac{\omega^{2} \tau}{1+(\omega \tau)^{2}}, \quad G=\left(\Sigma_{i} \nu_{i}^{2} / c_{A_{i}}\right)^{-1}
$$

mit

$$
\begin{aligned}
a_{\mathrm{p}} & : \text { thermischer Ausdehnungskoeffizient } \\
C_{\mathrm{p}} & : \text { Wärmekapazität bei konstantem Druck } \\
\Delta H & : \text { molare Reaktionsenthalpie } \\
\Delta V & : \text { molares Reaktionsvolumen } \\
\nu_{i}, c_{A_{i}} & : \text { Stöchiometriefaktoren und Konzentrationen der } \\
& \text { an der Gleichgewichtsreaktion beteiligten Moleküle } A_{i} .
\end{aligned}
$$


Die Form des Terms lässt sich aus Gl. (3.13) unter Annahme einer periodischen Konzentrationsschwankung herleiten; sie entspricht der Laplace-Transformierten des exponentiellen Abfalls.

In der $(\alpha \lambda)$-Darstellung erhält man für Gl. (3.15) einen Debye-Spektralterm

$$
(\alpha \lambda)_{\mathrm{ex}}=A_{\mathrm{D}} \frac{\omega \tau}{1+(\omega \tau)^{2}}
$$

mit

$$
A_{\mathrm{D}}=\frac{\pi c_{\mathrm{s}}^{2} \rho}{R T} G\left(\Delta V-\frac{a_{\mathrm{p}}}{\rho C_{\mathrm{p}}} \Delta H\right)^{2} .
$$

Die Amplitude $A_{\mathrm{D}}$ beschreibt die Kopplung zwischen Schallwelle und chemischer Reaktion und erlaubt Rückschlüsse auf Reaktionsvoluminen und Reaktionsenthalpie.

\subsection{Die Theorie von Bhattacharjee und Ferrell}

Wie bereits in Abschnitt 2.2.3 beschrieben, treten bei einem Phasenübergang zweiter Ordnung kritische Fluktuationen von thermodynamischen Ordnungsparametern in der Nähe der kritischen Temperatur $T_{\mathrm{c}}$ auf.

Die Wärmekapazität $C_{\mathrm{p}}$ lässt sich durch ein Potenzgesetz der Form

$$
C_{\mathrm{p}} \sim\left|\frac{T-T_{\mathrm{c}}}{T_{\mathrm{c}}}\right|^{-\tilde{\alpha}_{0}}, \quad \tilde{\alpha}_{0}=0,11
$$

mit dem kritischen Exponenten der Wärmekapazität $\tilde{\alpha}_{0}$ darstellen. Im Falle der Wärmekapazität ist dieses Verhalten auf den Anstieg der Fluktuation der Enthalpie $H$ gemäß Gl. (2.5) zurückzuführen [49].

Einhergehend mit dem Anwachsen der Fluktuationen am kritischen Punkt kann der Anstieg der Korrelationslänge $\xi$ der Fluktuationen und die Vergrößerung der charakteristischen Abklingzeit $\tau$ nach einem Potenzgesetz

$$
\xi=\xi_{0}\left|\frac{T-T_{\mathrm{c}}}{T_{\mathrm{c}}}\right|^{-\tilde{\nu}}, \tau^{-1}=\Gamma_{0}\left|\frac{T-T_{\mathrm{c}}}{T_{\mathrm{c}}}\right|^{-z_{0} \tilde{\nu}}
$$

mit

$\Gamma_{0}:$ Relaxationsratenamplitude

$\tilde{\nu}=3,05$ : kritischer Exponent der Korrelationslänge

$z_{0}=0,63:$ Dynamikexponent.

beschrieben werden. Eine Vergrößerung der Abklingzeit $\tau$ führt zu einer Verlangsamung aller Prozesse, die an das kritische Verhalten gekoppelt sind, dem "Critical Slowing Down“. 
Dieser Aspekt wurde bereits in Gl. (2.4) aus der Ganzburg-Lindau-Theorie [55, 88] hergeleitet.

Die Ordnungsparameterfluktuationen haben einen Einfluss auf die Ultraschallabsorption. Fluktuationen der Dichte - damit auch des Volumens - koppeln an die Druckschwankungen einer einfallenden Schallwelle an. Die daraus resultierende Dissipation liefert einen Beitrag zur Exzessdämpfung.

In [33] geben Bhattacharjee und Ferrell eine Herleitung der dynamischen Skalierungstheorie zur Beschreibung des Beitrages von kritischen Fluktuationen zur Ultraschallabsorption an. Diese Überlegungen sollen hier kurz skizziert werden.

Bhattacharjee und Ferrell gehen von der Laplaceschen Annahme [74] aus, dass die schnelle Kompression und Dilatation einer Schallwelle zu einer nicht vernachlässigbaren lokalen Temperaturänderung führt.

Nach [14] und [52] führt dieses zu einem komplexwertigen additiven Term in der Schallgeschwindigkeit

$$
c_{\mathrm{s}}(\omega, T)=c_{\mathrm{s}}(\omega=0, T)+c_{\mathrm{s}, 1} \frac{C_{0}}{C_{\mathrm{p}}(\omega, T)}
$$

mit der frequenzabhängigen komplexwertigen Wärmekapazität $C_{\mathrm{p}}(\omega, T)$ und den Konstanten $c_{\mathrm{s}, 1}$ und $C_{0}$. Der Imaginärteil dieses Ausdrucks entspricht dem Absorptionskoeffizient $\alpha$.

Bhattacharjee und Ferrell teilen die Wärmekapazität in einen nichtkritischen Hintergrundanteil $C_{1}$ und einen kritischen Teil $C(\omega)$ auf, so dass

$$
C_{\mathrm{p}}(\omega)=C_{1}+C(\omega) \quad \text { mit } \quad C(\omega) \sim C(\omega=0)(1+i \omega \tau),
$$

wobei $\tau$ die mittlere Reaktionszeit der Fluktuationen darstellt. Es wird gezeigt, dass

$$
C(\omega=0) \sim t^{-\tilde{\alpha}_{0}}
$$

mit der reduzierten Temperatur

$$
t=\left|\frac{T-T_{\mathrm{c}}}{T_{\mathrm{c}}}\right|
$$

und dem in Gl. (3.18) eingeführten kritischen Exponenten der Wärmekapazität $\tilde{\alpha}_{0}$ ist. Die Relaxation der kritischen Fluktuationen $[32,66]$ wird durch Diffusion mit der Diffusionskonstanten

$$
D=\frac{k_{\mathrm{B}} T}{6 \pi \eta_{\mathrm{s}} \xi}
$$

bewirkt, wobei

$$
\begin{aligned}
k_{\mathrm{B}} & : \text { Boltzmann-Konstante } \\
\eta_{\mathrm{s}} & : \text { Scherviskosität } \\
\xi & : \text { Korrelationslänge. }
\end{aligned}
$$


Damit ergibt sich die charakteristische Relaxationsrate

$$
\Gamma(t)=\frac{2 D}{\xi^{2}}=\Gamma_{0} t^{z_{0} \tilde{\nu}}=\Gamma_{0}\left|\frac{T-T_{\mathrm{c}}}{T_{\mathrm{c}}}\right|^{z_{0} \tilde{\nu}} .
$$

mit

$\Gamma_{0}:$ Relaxationsratenamplitude

$z_{0}:$ Dynamikexponent

$\tilde{\nu}$ : kritischer Exponent der Korrelationslänge.

Durch Ersetzen der Temperaturabhängigkeit in Gl. (3.22) durch die charakteristische Relaxationsrate aus Gl. (3.25) erhält man

$$
C(\omega=0) \sim t^{-\tilde{\alpha}_{0}} \Rightarrow C(\Gamma, \omega=0) \sim \Gamma^{-\tilde{\alpha}_{0} / z_{0} \tilde{\nu}}, \quad \frac{\tilde{\alpha}_{0}}{z_{0} \tilde{\nu}}=0,057 .
$$

Die Frequenzabhängigkeit von $C$ an der kritischen Temperatur mit $\Gamma=0$ lässt sich aus der Modenkopplungstheorie gewinnen:

$$
C(\Gamma=0, \omega)=\left(\frac{-i \omega}{a}\right)^{-\tilde{\alpha}_{0} / z_{0} \tilde{\nu}}
$$

mit der Konstanten $a$. Damit ergibt sich für den Dämpfungsexponenten $\alpha$ nach Gl. (3.21) und Gl. (3.27)

$$
\begin{aligned}
\alpha \sim \omega \operatorname{Im} C_{\mathrm{p}}(\omega)^{-1} & \approx-\frac{\omega}{\left(\operatorname{Re} C_{\mathrm{p}}\right)^{2}} \operatorname{Im} C(0, \omega) \\
& \approx-\frac{\tilde{\alpha}_{0} \pi}{2 z_{0} \tilde{\nu} a} \frac{\omega^{2}}{\left(\operatorname{Re} C_{\mathrm{p}}\right)^{2}}\left(\frac{a}{\omega}\right)^{1+\tilde{\alpha}_{0} / z_{0} \tilde{\nu}} \\
\Rightarrow\left(\alpha / f^{2}\right)_{\mathrm{ex}}^{\mathrm{cr}} & \sim f^{-1-\frac{\tilde{\alpha}_{0}}{z_{0}}}
\end{aligned}
$$

Mit Gl. (3.26) folgt

$$
\left(\alpha / f^{2}\right)_{\mathrm{ex}}^{\mathrm{cr}} \sim f^{-1,057}, \quad(\alpha \lambda)_{\mathrm{ex}}^{\mathrm{cr}} \sim f^{-0,057} .
$$

Die Ultraschallabsorption $\left(\alpha / f^{2}\right)_{\mathrm{ex}}$ in der Nähe des kritischen Punktes verhält sich nahezu proportional zur Frequenz der Schallwelle. In der $(\alpha \lambda)_{\mathrm{ex}}$-Darstellung liefert der kritische Anteil daher einen nahezu konstanten Beitrag im gesamten Frequenzbereich.

Bislang wurde noch nicht die Temperaturabhängigkeit der Absorption betrachtet. Die temperatur- und frequenzabhängige Form wird gegeben durch

$$
(\alpha \lambda)_{\mathrm{ex}}^{\mathrm{cr}}=A(T) F(\Omega) \quad \operatorname{mit} A(T)=\tilde{A}(T) \cdot \Gamma^{-\tilde{\alpha}_{0} / z_{0} \tilde{\nu}} .
$$

mit

$$
\begin{aligned}
\tilde{A}(T) & : \text { temperaturabhängige Amplitude } \\
\tilde{\alpha}_{0}, z_{0}, \tilde{\nu} & : \text { kritische Exponenten } \\
F(\Omega) & : \text { Skalierungsfunktion } \\
\Gamma & : \text { Relaxationsrate der kritischen Fluktuationen } \\
\Omega=\omega / \Gamma & : \text { reduzierte Frequenz. }
\end{aligned}
$$


Bhattacharjee und Ferrell geben für verschiedene Phasenübergänge jeweils leicht unterschiedliche Skalierungsfunktionen mit ähnlichen Spektralformen an.

Die Skalierungsfunktion gibt das Verhältnis der Zusatzabsorption an der kritischen Temperatur zu einer Temperatur in dessen Nähe an

$$
F(\Omega)=\frac{(\alpha \lambda)_{\mathrm{ex}}^{\mathrm{cr}}(T)}{(\alpha \lambda)_{\mathrm{ex}}^{\mathrm{cr}}\left(T_{\mathrm{c}}\right)}
$$

wenn $A(T)$ in der Nähe von $T_{\mathrm{c}}$ nur schwach von $T$ abhängt. Es gilt

$$
\lim _{t \rightarrow \infty} F(\Omega)=0, \quad \lim _{t \rightarrow 0} F(\Omega)=1, \quad t=\left|\frac{T-T_{\mathrm{c}}}{T_{\mathrm{c}}}\right| .
$$

Als empirische Skalierungsfunktion für die kritischen Fluktuationen am Lipid-Phasenübergang von der gelförmigen Phase in die fluide Phase wird die Funktion

$$
F(\Omega)=\sqrt{\frac{2}{\Omega}}\left(\left(1+\Omega^{2}\right)^{1 / 4} \cos \left(\frac{\arctan \Omega}{2}\right)-1\right)
$$

angenommen [42]. Diese Funktion wurde von Bhattacharjee und Ferrell ursprünglich für den Phasenübergang in Flüssigkristallen auf Basis der Mean-Field-Theorie [17] berechnet. Gl. (3.33) gibt lediglich eine Näherung für die Lösung der zu Grunde liegenden Gleichungen an, die im Allgemeinen nicht geschlossen gelöst werden können.

Mit Gl. (3.28) und Gl. (3.30) folgt für die Exzessdämpfung durch die kritischen Fluktuationen

$$
A(T) F(\Omega) \sim f^{-\tilde{\alpha}_{0} / z_{0} \tilde{\nu}}
$$

Mit der Einführung der kritischen Amplitude $A_{\mathrm{BF}}$ folgt

$$
(\alpha \lambda)_{\mathrm{ex}}^{\mathrm{cr}}=A_{\mathrm{BF}}(T) F(\Omega) f^{-\tilde{\alpha}_{0} / z_{0} \tilde{\nu}} .
$$

Diese Absorption kann ebenfalls durch physikalische Größen beschrieben werden

$$
(\alpha \lambda)_{\mathrm{ex}}^{\mathrm{cr}}=\frac{\pi^{2} \frac{\tilde{\alpha}_{0}}{z_{0} \tilde{\nu}}(\Delta C)}{2 T_{\mathrm{c}}}\left(\frac{\Omega_{1 / 2} \Gamma}{2 \pi}\right)^{-\tilde{\alpha}_{0} / z_{0} \tilde{\nu}} \cdot \frac{c_{\mathrm{s}}^{2} g^{2}}{C_{\mathrm{p}}^{2}} f^{-\tilde{\alpha}_{0} / z_{0} \tilde{\nu}},
$$

Es wird ein Kopplungsparameter eingeführt mit

$$
g=\rho_{\mathrm{c}} C_{\mathrm{p}}\left(\frac{d T_{\mathrm{c}}}{d p}-\frac{T a_{\mathrm{p}}}{\rho C_{\mathrm{p}}}\right)
$$

mit

$\rho_{\mathrm{c}}:$ Dichte an der kritischen Temperatur

$a_{\mathrm{p}}$ : thermischer Expansionskoeffizient.

$\Delta C$ entspricht dem kritischen Anteil der Wärmekapazität $C(\omega=0)$ in Gl. (3.21) und Gl. (3.22). 


\section{Messverfahren der Ultraschallabsorptionsspektrometrie}

Die Ultraschallabsorptionsspektrometrie ist ein geeignetes Verfahren, um die Kinetik schneller molekularer Prozesse zu untersuchen. Im vorhergehenden Kapitel wurden die Einflüsse auf das Absorptionsspektrum von Einzelzeitrelaxationen und kritischen Fluktuationen am Phasenübergang beschrieben. Für Lipidsysteme findet man die Relaxationszeiten dieser Beiträge im Bereich von einigen Millisekunden bis in den Nanosekundenbereich. Es werden daher im Rahmen dieser Arbeit Spektren im Frequenzbereich von $100 \mathrm{kHz}$ bis hin zu $2 \mathrm{GHz}$ aufgenommen. Die Überlegungen des vorherigen Kapitels zeigen, dass einzelne Prozesse messbare Beiträge über mehrere Frequenzdekaden liefern. Um diese Prozesse separieren und charakterisieren zu können, ist die Aufnahme eines kompletten Spektrums in den meisten Fällen unerlässlich.

In diesem Kapitel werden die spektroskopischen Messverfahren vorgestellt, die im Rahmen dieser Arbeit Verwendung finden: Das Resonatorverfahren, aus dem auch die Schallgeschwindigkeitsmessung hervorgeht, und das Schwingungspulstransmissionsverfahren. Begonnen werden soll aber zuvor mit einer kurzen Zusammenfassung über die Eigenschaften piezoelektrischer Wandler, die bei dem hier benutzten Verfahren als Sende- und Empfangseinheiten verwendet werden.

\subsection{Der piezoelektrische Wandler}

Für die Erzeugung von hochfrequenten Schallwellen werden piezoelektrische Wandler eingesetzt. Die Elementarzellen piezoelektrischer Kristalle wie Quarz $\left(\mathrm{SiO}_{2}\right)$ und Lithiumniobat $\left(\mathrm{LiNbO}_{3}\right)$ weisen eine polare Achse auf. Die Atome sind zwar rotationssymmetrisch angeordnet, jedoch sind die Achsenrichtungen nicht gleichwertig, wie die vereinfachte Einheitszelle in Abb. 4.1 (b) zeigt. Durch diese Polarität reagiert der Kristall sensitiv auf die Richtung äußerer elektrischer Felder. Entsprechend der Feldrichtung reagiert der Kristall im Innern mit einer Deformation, die ein Gegenfeld gemäß

$$
E=\delta \frac{\Delta x}{x}
$$

erzeugt, wobei $\delta$ der piezoelektrische Koeffizient und $\Delta x / x$ die relative Längenänderung ist [36]. 

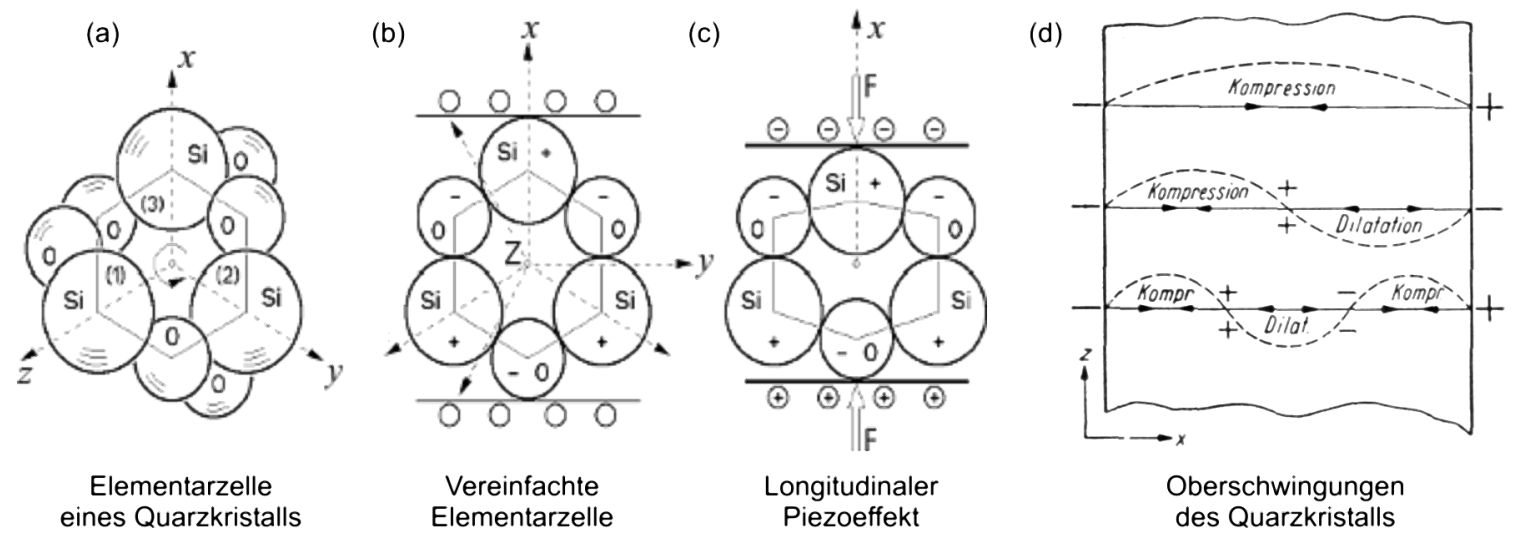

Abbildung 4.1: Der piezoelektrische Effekt. (a) Darstellung der dreidimensionalen Elementarzelle eines Quarzkristalls; (b) verdeutlicht die Symmetrie des Kristalls. (c) veranschaulicht den longitudinalen Piezoeffekt. (d) zeigt, dass bei Anlegen eines äußeren Potenzials nur ungerade Oberschwingungen angeregt werden können.

Schneidet man einen Quarzwandlerkristall in kristallographischer $x$-Richtung, wie in Abb. 4.1 (c) dargestellt, so kann der Kristall als Dickenschwinger eingesetzt werden.

Bei Anlegen eines elektrischen Wechselfeldes wird nach Gl. (4.1) der Kristall zu Längenänderungen entlang der Polarisationsachse und damit zur Abstrahlung von Schallwellen angeregt. Dieser Prozess ist reversibel, d. h. eine Deformation des Kristalls durch einfallende Schallwellen führt zur Ausbildung eines elektrischen Feldes. Ein piezoelektrischer Kristall kann daher auch als Empfangswandler für Schallwellen eingesetzt werden. Die Effizienz der Konversion von mechanischer in elektrische Energie (und umgekehrt) liegt bei etwa $1 \%$.

Die Resonanzfrequenzen werden gegeben durch

$$
f_{\mathrm{w}, m}=(2 m+1) \frac{c_{\mathrm{s}, \mathrm{w}}}{2 d_{\mathrm{w}}}, \quad \forall m \in \mathbb{N}
$$

mit

$$
\begin{aligned}
f_{\mathrm{w}, m} & : m \text {-te Oberschwingung der Dickenresonanz des Wandlers } \\
c_{\mathrm{s}, \mathrm{w}} & : \text { Schallgeschwindigkeit im piezoelektrischen Kristall } \\
d_{\mathrm{w}} & : \text { Dicke des Kristalls. }
\end{aligned}
$$

Es können nur Oberschwingungen $(2 n+1)$-ter Ordnung angeregt werden, wie Abb. 4.1 zeigt. Neben den Dickenresonanzen treten Scherresonanzen und deren Oberschwingungen auf. Nach [7] lässt die Grundresonanz der Scherschwingung angeben als

$$
f_{\mathrm{w}, 0}^{\mathrm{s}}=0,6 \cdot f_{\mathrm{w}, 0}
$$

Um ein homogenes elektrisches Feld zu erzeugen, werden die Wandlervorder- und -rückseite mit einer Goldschicht bedampft. Die Kontaktierung der Vorderseite, die dem Messvolumen zugewandt ist, erfolgt vielfach an den Wandlerrändern über Leitsilber und einen federnden Metallring um den Wandler. Die Rückseite wird zentral von einem Metallstift oder einer Bronzefeder kontaktiert. 


\subsection{Das Resonatorverfahren}

Für den Frequenzbereich unterhalb von $20 \mathrm{MHz}$ wird das Resonatorverfahren zur Bestimmung des Absorptionskoeffizienten angewandt. Es stellt im Rahmen dieser Arbeit das meistgenutzte Verfahren dar. Aus diesem Grunde sollen im folgenden Kapitel die theoretischen und messtechnischen Grundlagen ausführlich beschrieben werden.

\subsubsection{Der ideale Resonator}

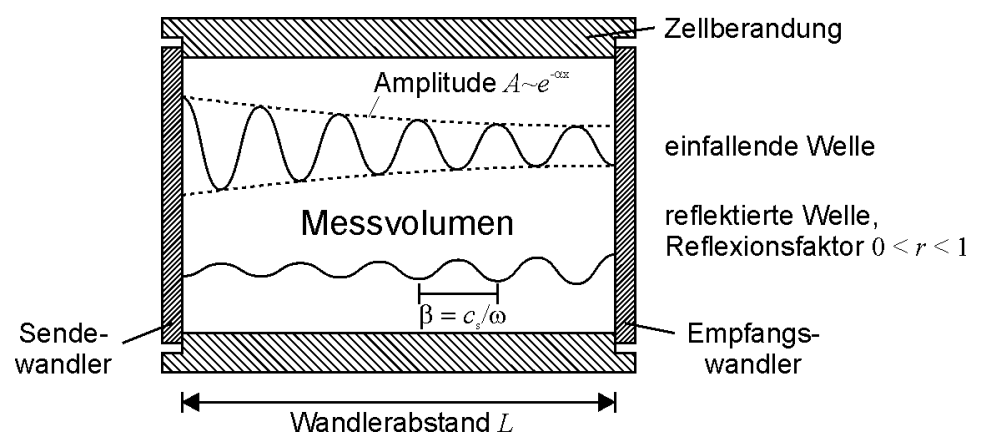

Abbildung 4.2: Wellenausbreitung im Resonator. In der Abbildung ist die schematische Wellenausbreitung in einem Resonator dargestellt.

Für die theoretischen Überlegungen sei angenommen, dass die Schallenergie vollständig in das Probenvolumen abgestrahlt wird. Die Wandler des idealen Resonator besitzen daher eine planare Geometrie bei unendlicher lateraler Ausdehnung, so dass keine Beugung an den Rändern auftreten kann, und einen schallharten Abschluss an der Wandlerrückseite bei infinitesimaler Wandlerdicke.

Energiedissipation kann unter diesen Voraussetzungen nur im Probenvolumen stattfinden. Das Volumen wird durch die Sende- und Empfangswandler begrenzt, die sich im Abstand $L$ voneinander befinden. Die im Folgenden verwendeten Größen sind in Abb. 4.2 schematisiert.

Für die Schalldruckamplitude am Empfangswandler erhält man bei kontinuierlicher Anregung am Empfangswandler durch Überlagerung von ein- und auslaufenden Wellen

$$
\begin{aligned}
p_{\mathrm{e}} & =p_{\mathrm{s}} e^{-\gamma L} e^{i \omega t}+p_{\mathrm{s}} r e^{-\gamma L} e^{i \omega t} \\
& +p_{\mathrm{s}} r^{2} e^{-3 \gamma L} e^{i \omega t}+p_{\mathrm{s}} r^{3} e^{-3 \gamma L} e^{i \omega t}+\ldots \\
& =p_{\mathrm{s}}(1+r) e^{-\gamma L} e^{i \omega t} \sum_{n=0}^{\infty}\left(r^{2} e^{-2 \gamma L}\right)^{n} \\
& =p_{\mathrm{s}}(1+r) \frac{e^{-\gamma L} e^{i \omega t}}{1-r^{2} e^{-2 \gamma L}}
\end{aligned}
$$

mit

$$
\begin{aligned}
\omega=2 \pi f: \text { Kreisfrequenz der Schallwelle } \\
p_{\mathrm{s}}, p_{\mathrm{e}}: \text { Schalldruckamplitude an Sende- und Empfangswandler } \\
\gamma=\alpha+i \beta: \text { Komplexer Absorptionskoeffizient mit } \\
\alpha: \text { Dämpfungsexponent und } \beta=c_{\mathrm{s}} / \omega: \text { Wellenzahl. }
\end{aligned}
$$


Nimmt man weiterhin eine schallharte Reflexion an den Grenzflächen zwischen Flüssigkeit und Wandler mit $|r|=1$ an, so folgt

$$
\begin{aligned}
p_{\mathrm{e}} & =p_{\mathrm{s}}(1+r) \frac{e^{-\gamma L} e^{i \omega t}}{1-r^{2} e^{-2 \gamma L}}, \\
& =2 p_{\mathrm{s}} \frac{e^{-\gamma L} e^{i \omega t}}{1-e^{-2 \gamma L}}=2 p_{\mathrm{s}} \frac{1}{e^{\gamma L}-e^{-\gamma L}} \\
& =2 p_{\mathrm{s}} \frac{1}{\sinh \gamma L} .
\end{aligned}
$$

Damit folgt für eine Übertragungsfunktion $T(f)$ :

$$
T(f)=\frac{p_{\mathrm{e}}}{p_{\mathrm{s}}} \sim \frac{1}{\sinh \gamma L} .
$$

Der Betrag der Funktion lässt sich durch Zerlegung in Real- und Imaginärteil mit $\sinh ^{2}(\gamma L)=\sinh ^{2}(\alpha L)+\sin ^{2}(\beta L)$ gewinnen:

$$
|T(f)| \sim \frac{1}{\sqrt{\sinh ^{2}(\alpha L)+\sin ^{2}(\beta L)}} .
$$

Aus der Periodizität der Sinusfunktion $\sin (n \pi)=0, \forall n \in \mathbb{N}_{0}$, lassen sich die äquidistanten Maxima der Funktion ableiten:

$$
\begin{array}{r}
\sin (\beta L)=0 \Rightarrow \quad \beta L=\left(\frac{2 \pi f_{n}}{c_{\mathrm{s}}}\right) L=n \cdot \pi \\
\Rightarrow f_{n}=\frac{c_{\mathrm{s}}}{2 L}
\end{array}
$$

Für $(\alpha L) \ll 1$ kann die Form der Kurve für kleine $\delta f=f-f_{n}$ in der Nähe der Resonanzen durch eine Taylor-Entwicklung gewonnen werden. Bei Messungen an wässrigen Lösungen ist dieses im Allgemeinen erfüllt. Es gilt:

$$
\begin{aligned}
\sinh (\alpha L) & \approx \alpha L \\
\sin (\beta L) & =\sin \left(\frac{2 \pi\left(f_{n}+\delta f\right)}{c_{\mathrm{s}}} L\right)=\sin \left(\frac{\pi \delta f}{c_{\mathrm{s}}} L\right) \\
& \approx \frac{2 \pi \delta f}{c_{\mathrm{s}}} L .
\end{aligned}
$$

Die resultierende Kurve

$$
\left|T\left(f_{n}\right)\right| \sim \frac{1}{\sqrt{(\alpha L)^{2}+\left(\frac{2 \pi \delta f}{c_{\mathrm{s}}} L\right)^{2}}}
$$

hat die Form einer Lorentzfunktion. 
In einem Frequenzabstand $\delta f=\Delta f_{n} / 2$ von $f_{n}$ erhält man einen Abfall der Leistung auf die Hälfte, entsprechend einer Reduktion der Schalldruckamplitude um den Faktor $1 / \sqrt{2}$. Man erhält über die Gleichung

$$
\left|\frac{T\left(f_{n}+(\Delta f / 2)\right)}{T\left(f_{n}\right)}\right|=\frac{\sqrt{(\alpha L)^{2}}}{\sqrt{(\alpha L)^{2}+\left(\frac{\pi(\Delta f) L}{c_{\mathrm{s}}}\right)^{2}}} \stackrel{!}{=} \frac{1}{\sqrt{2}}
$$

eine Verbindung zwischen der Halbwertsbreite $\Delta f_{n}$ der $n$-ten Resonanz und dem Dämpfungskoeffizienten $\alpha$. Es ist

$$
\alpha\left(f_{n}\right)=\frac{\pi}{c_{\mathrm{s}}} \Delta f_{n} \quad \text { bzw. } \quad(\alpha \lambda)\left(f_{n}\right)=\pi \frac{\Delta f_{n}}{f_{n}} .
$$

\subsubsection{Der reale Resonator}

\section{Apparative Verluste}

Zur Beschreibung der Resonatoreigenschaften wird die Güte $Q$ eingeführt. Sie gibt das Verhältnis aus reversibel im Schallfeld gespeicherter Energie $E_{\mathrm{r}}$ und der dissipierten Energie $E_{\mathrm{d}}$ an:

$$
Q:=2 \pi \frac{E_{\mathrm{r}}}{E_{\mathrm{d}}}
$$

Experimentell lässt sich die Güte einer Resonanz als Quotient aus Mittenfrequenz und Halbwertsbreite bestimmen:

$$
Q=\frac{f_{n}}{\Delta f}
$$

Neben der Flüssigkeitsdämpfung treten im realen Resonator weitere apparative Verluste auf, die zu einer Erhöhung der Halbwertsbreite und zu einer Reduktion der Güte $Q$ führen. Es gilt bei nicht zu geringen $Q_{\mathrm{fl}}$ und $Q_{\mathrm{app}}$

$$
Q_{\mathrm{ges}}^{-1}=Q_{\mathrm{fl}}^{-1}+Q_{\mathrm{app}}^{-1}
$$

wobei

$$
\begin{aligned}
Q_{\text {ges }} & : \text { Güte des Gesamtsystems } \\
Q_{\mathrm{fl}} & : \text { Beitrag der Flüssigkeitsverluste zur Güte } \\
Q_{\mathrm{app}} & : \text { Beitrag der apparativen Verluste zur Güte. }
\end{aligned}
$$

Nach Labhardt und Schwarz [72, 73] kann der apparative Beitrag durch Beugungs- und Wandlerverluste beschrieben werden. Die Beugungsverluste werden für biplanare Resonatoren angegeben durch

$$
(\alpha \lambda)_{\text {beug }}=\frac{0,147}{\beta_{\omega}} \cdot\left(\frac{c_{\mathrm{s}}}{R}\right)^{3} \cdot \frac{1}{f_{n}^{2}}
$$


wobei $R$ der Radius des Resonators ist und $\beta_{\omega}$ die spezifische Eingangsimpedanz der Zellberandung ist. Für $\alpha \ll \beta$ wird $\beta_{\omega}$ angegeben durch

$$
\beta_{\omega}=\frac{\omega \rho_{\mathrm{fl}}}{\beta} \frac{1}{Z_{\mathrm{b}}} \approx \frac{Z_{\mathrm{fl}}}{Z_{\mathrm{b}}}=\frac{c_{\mathrm{s}, \mathrm{fl}} \cdot \rho_{\mathrm{fl}}}{c_{\mathrm{s}, \mathrm{b}} \cdot \rho_{\mathrm{b}}}
$$

mit

$$
\begin{aligned}
& \omega=2 \pi f: \text { Kreisfrequenz der Schallwelle } \\
& \beta: \text { Wellenzahl } \\
& \rho_{\mathrm{fl}}, \rho_{\mathrm{b}}: \text { Dichte von Flüssigkeit und Zellberandung } \\
& Z_{\mathrm{fl}}, Z_{\mathrm{b}}: \text { Impedanz von Flüssigkeit und Zellberandung } \\
& c_{\mathrm{s}, \mathrm{f}}, c_{\mathrm{s}, \mathrm{b}}: \text { Schallgeschwindigkeit in Flüssigkeit und Zellberandung. }
\end{aligned}
$$

Gl. (4.13) zeigt, dass die Dämpfung $(\alpha \lambda)_{\text {beug }} \sim 1 / f^{2}$ zu kleiner werdenden Frequenzen ansteigt. Die Beugungseffekte treten daher besonders stark bei niedrigen Frequenzen in Erscheinung.

Die Verluste, die durch den Wandler hervorgerufen werden, lassen sich im Allgemeinen nicht geschlossen durch Gleichungen angeben. Es handelt sich hierbei unter anderem um Abstrahlung der Schallenergie in den Außenraum des Resonators. Dieser Effekt tritt in der Nähe der Scher- und Dickenresonanz des Wandlerkristalls auf.

Um die Beiträge aus Beugungs- und Wandlerverlusten bestimmen zu können, wird vor jeder Messung an der zu untersuchenden Flüssigkeit eine Messung mit einer Referenz durchgeführt, deren physikalische Eigenschaften bekannt sind. Referenzlösung und Messsubstanz sollten nach Gl. (4.13) in Schallgeschwindigkeit, Dichte übereinstimmen, um vergleichbare Bedingungen für die Messung der Beugungsverluste zu gewährleisten. Es gilt

$$
Q_{\mathrm{ges}}^{-1}=Q_{\mathrm{fl}}^{-1}+Q_{\mathrm{app}}^{-1} \quad \text { und } \quad Q_{\mathrm{ges}, \mathrm{ref}}^{-1}=Q_{\mathrm{fl}, \mathrm{ref}}^{-1}+Q_{\mathrm{app}}^{-1}
$$

mit

$$
\begin{gathered}
Q_{\text {ges }}, Q_{\text {ges,ref }}: \text { Gesamtgüte von Substanz- und Referenzmessung } \\
Q_{\mathrm{fl}}^{-1}, Q_{\mathrm{fl}, \mathrm{ref}}^{-1}: \text { Dämpfungsbeitrag von Messsubstanz und Referenz } \\
Q_{\mathrm{app}}^{-1}: \text { Dämpfungsbeitrag von apparativen Verlusten. }
\end{gathered}
$$

Wird $Q_{\mathrm{fl}, \mathrm{ref}}^{-1}$ als bekannt aus der Theorie vorausgesetzt, so folgt

$$
Q_{\mathrm{fl}}^{-1}=Q_{\mathrm{ges}}^{-1}-Q_{\mathrm{ges}, \mathrm{ref}}^{-1}+Q_{\mathrm{fl}, \mathrm{ref}}^{-1}
$$

Die Verluste durch Flüssigkeitsdämpfung lassen sich durch diese Beziehung von der Gesamtdämpfung trennen. Weichen die Dämpfungen von Referenzlösung und Messsubstanz stark voneinander ab, so ist eine Korrektur der Messwerte nach Kononenko [68] vorzunehmen. Jedoch wurden alle Messungen an wässrigen Lösungen durchgeführt, so dass Dichte $\rho_{\mathrm{fl}}$ und Schallgeschwindigkeit $c_{\mathrm{s}, \mathrm{f}}$ nur geringe Abweichungen aufwiesen:

$$
\rho_{\mathrm{fl}} \approx \rho_{\mathrm{fl}, \mathrm{ref}} \quad \text { und } \quad c_{\mathrm{s}, \mathrm{fl}} \approx \rho_{\mathrm{fl}, \mathrm{ref}} .
$$




\subsubsection{Einfluss der Wandlereigenschaften}

\section{Frequenzabhängigkeit der Resonanzeigenschaften}

In der Theorie des idealen Resonators wird die Wandlerdicke infinitesimal sowie der Flüssigkeit/Wandler-Übergang als schallhart angenommen. Im realen Fall besitzen aber die Wandler eine endliche Dicke. Aufgrund eines Reflexionsfaktors $|r|<1$, kann ein Teil der Schallwelle in den Wandler eindringen, wodurch die Laufstrecke der Welle verlängert wird. In der Mitte zweier Dickenresonanzen des Quarzes bekommt man eine $\lambda / 4$ Transformation, die zu einer schallharten Reflexion $|r|=1$ an der Grenzfläche von Wandler zu Flüssigkeit führt. Labhardt [72] führt einen Korrekturfaktor ein, der die Verlängerung der Wegstrecke beschreibt, und damit zu einer Änderung des Resonanzabstandes führt:

$$
f_{n}-f_{n-1}=\frac{c_{\mathrm{s}, \mathrm{f}}}{2 \pi L} \arccos \left(\frac{\left(g_{n}^{2}-1\right)\left(1-g_{n-1}^{2}\right)-4 g_{n} g_{n-1}}{\left(g_{n}^{2}+1\right)\left(g_{n-1}^{2}+1\right)}\right), n \in \mathbb{N}
$$

mit

$$
g_{n}=\frac{\rho_{\mathrm{fl}} c_{\mathrm{s}, \mathrm{fl}}}{\rho_{\mathrm{w}} c_{\mathrm{w}} \tan \left(\pi \frac{f_{n}}{f_{\mathrm{w}}}\right)}
$$

und

$f_{n}:$ Mittenfrequenz der $n$-ten Resonanz

$L:$ Zelllänge

$c_{\mathrm{s}, \mathrm{f}}, c_{\mathrm{s}, \mathrm{w}}:$ Schallgeschwindigkeit in Flüssigkeit und Wandler

$\rho_{\mathrm{fl}}, \rho_{\mathrm{w}}:$ Dichte der Flüssigkeit und des Wandlers

$f_{\mathrm{w}}$ : Resonanzfrequenz des Wandlers.

Wie aus Abb. 4.3 deutlich wird, besitzt der Resonator eine frequenzabhängige Sensitivität gegenüber den Amplituden der Zellresonanzen. Bei hohen Dämpfungen, die zu breiten Resonanzen führen, ist daher eine Anpassung der Frequenzabhängigkeit der Amplituden nötig. Nach [30] kann die Einhüllende der Amplitude gegeben werden durch

$$
E_{m}(f)=\frac{E_{m, 0} \cdot e^{-B f}}{1+\left(d \cdot \tan \left(\frac{\pi}{2}\left(\frac{f}{f_{\mathrm{q}, m}}+1\right)\right)\right)^{2}} .
$$

$E_{m}(f)$ besitzt wiederum die Form einer Lorentzkurve. Dabei wird die Amplitude gegeben durch $E_{\mathrm{m}, 0}$, die Halbwertsbreite durch den Parameter $d$ und die Mittenfrequenz durch die $m$-te Quarzresonanz $f_{\mathrm{q}, m}$. Der Term $e^{-B f}$ berücksichtigt zusätzlich Anteile, die durch elektrisches Übersprechen hervorgerufen werden.

\section{Radialmoden}

Aufgrund der endlichen Wandlerradien kann sich das Schallfeld im Wandler neben der Hauptmode auch in verschiedenen Radialmoden ausbreiten. Labhardt [72] gibt für die 


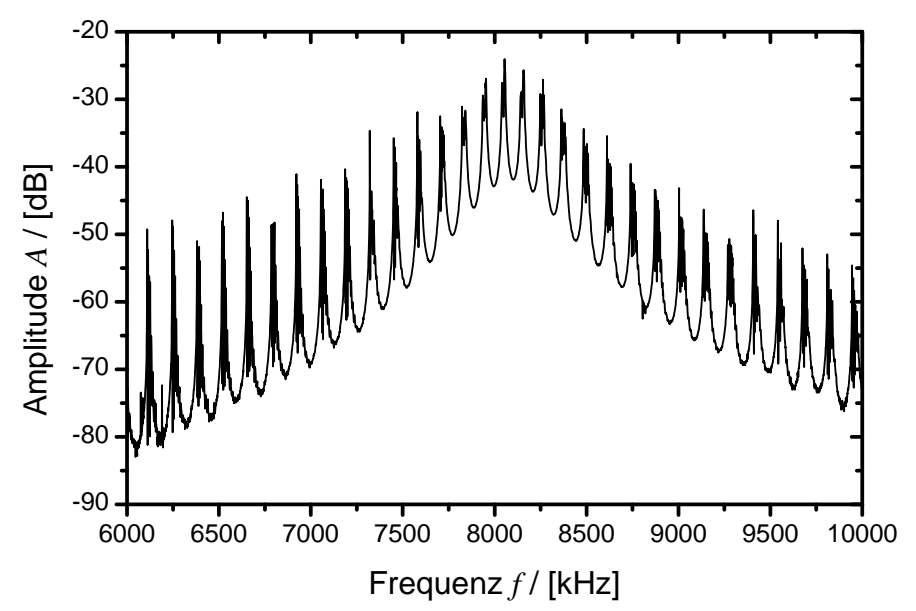

Abbildung 4.3: Spektrum des 8-MHz-Resonators in der Nähe der ersten Hauptresonanz. Es ist eine deutliche Abhängigkeit der Amplitude von der Frequenz zu erkennen. Die Einhüllende der Kurve wird durch GI. (4.17) gegeben.

Lage der $m$-ten Radialmode $f_{n}^{m}$ relativ zur Hauptmode $f_{n}$ im biplanaren Resonator die folgende Gleichung an:

$$
f_{n}^{m}-f_{n}=\left(\frac{c_{\mathrm{s}, \mathrm{H}}}{4 R}\right)^{2}\left(\frac{m(2 m-1)-1}{f_{n}}\right),
$$

wobei $R$ der Radius der Wandler ist. Der Abstand der Nebenmoden verringert sich mit zunehmender Resonanzfrequenz $f_{n}$. Das heißt, die Form der Resonanz der Hauptmode wird mit wachsender Frequenz zunehmend von Nebenmoden beeinflusst. Diese Störungen werden jedoch bei der abschließenden Auswertung, die in Abschnitt 4.2.6 beschrieben wird, berücksichtigt.

\section{Wandlergeometrie}

Von entscheidender Bedeutung für das Resonanzspektrum ist die Geometrie des Wandlers. Man unterscheidet im Allgemeinen drei Arten von Wandleranordnungen und Resonatortypen: biplanare Resonatoren, bikonkave Resonatoren und planar-konkave Resonatoren.

Der biplanare Resonator, d. h. ein Resonator, dessen Wandlerstirnflächen planar sind, ist bereits in den bisherigen Überlegungen behandelt worden. Der Nachteil dieser Anordnung besteht darin, dass der Resonator sehr empfindlich gegenüber Verkippungen ist. Befinden sich die Wandleroberflächen nicht genau parallel zueinander, dann wird ein Teil der Schallenergie in den Außenraum reflektiert, so dass dieser Effekt als apparative Zusatzdämpfung zu einer Verbreiterung der Resonanzen führt.

Auch ist im Falle der biplanaren Anordnung mit Beugungseffekten an den Rändern zu rechnen, da das Schallfeld - im Idealfall - gleichermaßen im Zentrum der Wandleroberfläche, sowie an den Rändern einfällt.

Der bikonkave Resonator reduziert Beugungseffekte. Die Idee hinter dem Einsatz eines bikonkaven Resonators ist die Fokussierung des Schallfeldes. Schallwellen, die auf einen 


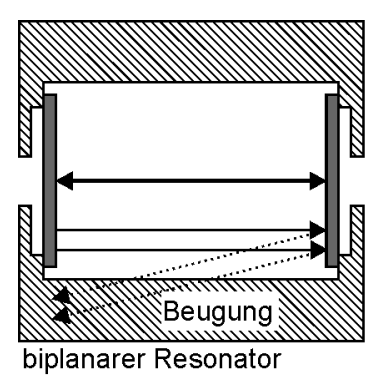

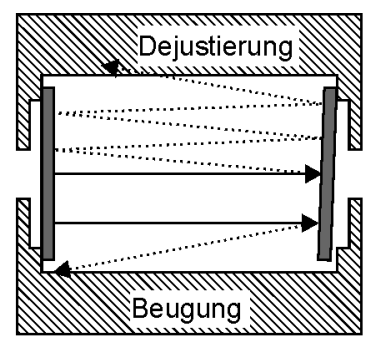

biplanarer Resonator, dejustiert

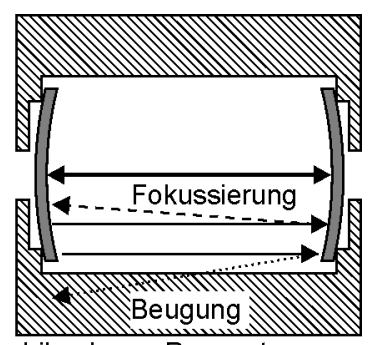

bikonkaver Resonator

Abbildung 4.4: Skizzierung der Wandlerverluste. Im biplanaren Resonator werden Rand,,strahlen" an den Wandlerrändern bei tiefen Frequenzen in den Außenraum gebeugt und tragen so zu einer Zusatzabsorption bei. Bei einer Dejustierung der Wandler werden auch zentrale Strahlen zunehmend in den Außenraum reflektiert. Der bikonkave Resonator hingegen fokussiert das Schallfeld, so dass Beugungsverluste und Verluste durch Wandlerdejustierung reduziert werden.

konkaven Wandler fallen, werden immer in Richtung des eingeschlossenen Messvolumens reflektiert. Dadurch wird sowohl die Beugung an den Rändern reduziert, sowie eine größere Toleranz bei der Wandlerjustierung erreicht.

Die konkave Geometrie begünstigt die Ausbreitung von Radialmoden, so dass man für jede Zellresonanz ein ausgeprägtes Modenspektrum erhält. Im Gegensatz zum biplanaren Resonator ist die Lage dieser Moden jedoch unabhängig von der Resonanzfrequenz. Nach [7] wird der Abstand gegeben durch

$$
f_{n}^{m}-f_{n}=m \frac{c}{\pi L} \arccos \left(1-\frac{L}{k}\right) .
$$

$k$ beschreibt hier den Krümmungsradius der Wandler, der im Normalfall in der Größenordnung von Metern liegt.

Der planar-konkave Resonator vereinigt die Eigenschaften des biplanaren und des bikonkaven Resonators. Auf der einen Seite zeigt er wenig Beugungverluste und eine gute Toleranz gegenüber Dejustierung, auf der anderen Seite ist sein Resonanzspektrum weniger ausgeprägt als beim bikonkaven Resonator. Für die Lage der Radialmoden kann angegeben werden [7]:

$$
f_{n}^{m}-f_{n}=m \frac{c}{\pi L} \arccos \left(\sqrt{1-\frac{L}{k}}\right) .
$$

\subsubsection{Aufbau des Messplatzes}

Abb. 4.5 zeigt den schematischen Aufbau eines Resonator-Messplatzes. Bei diesem Aufbau wird ein Netzwerkanalysator (2) zur Signalerzeugung und -analyse verwendet. Der Netzwerkanalysator, NWA, erzeugt ein kontinuierliches Signal $U_{\mathrm{S}}$, das über einen $3 \mathrm{~dB}$ Signalteiler (3) in ein Referenzsignal $U_{\mathrm{E}}$ und ein Messsignal $U_{\mathrm{M}}$ aufgeteilt wird. Das Referenzsignal wird wieder in den NWA zurückgeführt. Das Messsignal regt den Sendewandler 


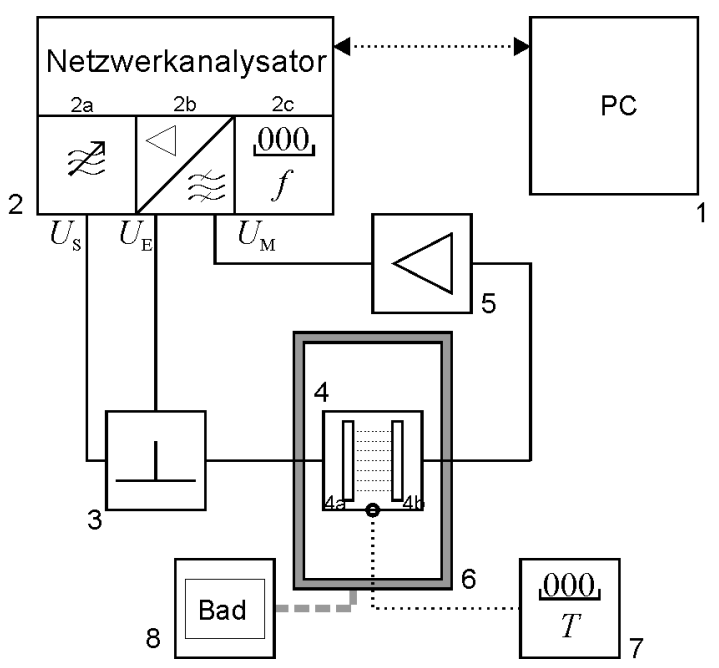

Abbildung 4.5: Blockschaltbild für einen Resonatormessplatz. Die durchgehende Linie symbolisiert den Weg des Messsignals, gepunktete Linien repräsentieren Mess- und Steuerleitungen. (1) Steuerrechner; (2) Netzwerkanalysator mit (2a) Generator; (2b) ZweikanalÜberlagerungsempfänger und (2c) Frequenzzähler; (3) 3dB-Signalteiler; (4) Resonator mit (4a) Sende- und (4b)-Empfangswandler; (5) regelbarer Verstärker; (6) äußere Thermostatierhülle; (7) Digitalthermometer mit externem Pt100-Messfühler; (8) Wasserbad zur Thermostatierung.

(4a) zu Schwingungen an, die in das Messvolumen abgestrahlt werden. Der Quarzempfänger (4b) wandelt anschließend die Schallwelle wieder in ein elektrisches Signal um, das über einen regelbaren Verstärker (5) zurück an den Netzwerkanalysator gegeben wird. Im NWA wird der Quotient $U_{\mathrm{M}} / U_{\mathrm{E}}$ gebildet, um die Übertragungsfunktion des Resonators bestimmen zu können. Über einen Steuerrechner (1) können die Messbereichsgrenzen und andere Parameter an den NWA gesendet werden, die für das Ausmessen der Resonanzen notwendig sind. Nach der Messung werden frequenzabhängige Amplituden- und Phaseninformationen an den Rechner gesendet und gespeichert. Ein temperierbares Wasserbad (8), das an die Thermostatierkanäle des Resonators und an eine isolierende Hülle um den Resonator herum angeschlossen ist, dient zum Einstellen der Messtemperatur. Mit Hilfe eines Digitalthermometers (7), das in Kontakt mit dem Messvolumen angebracht ist, wird regelmäßig die Temperatur kontrolliert.

\subsubsection{Die verwendeten Resonatoren}

\section{Der 1-MHz-Reflektor-Resonator}

Bei der Wahl des tieffrequenten Resonators muss neben anderen Parametern auch das Messvolumen aufgrund des hohen Preises für die zu untersuchenden Lipid/Peptid-Membranen beachtet werden. Für diese Messungen wird ein neuartiger 1-MHz-Reflektor-Resonator nach Polacek [92, 93] verwendet, der ein Zellvolumen $V \approx 30 \mathrm{ml}$ aufweist. Für bewährte Resonatoren in diesem Messbereich wie dem planar-konkaven 1-MHz-Resonator nach Telgmann [106, 107] wird eine wesentlich größere Probenmenge benötigt, so dass diese nicht verwendet werden können. 


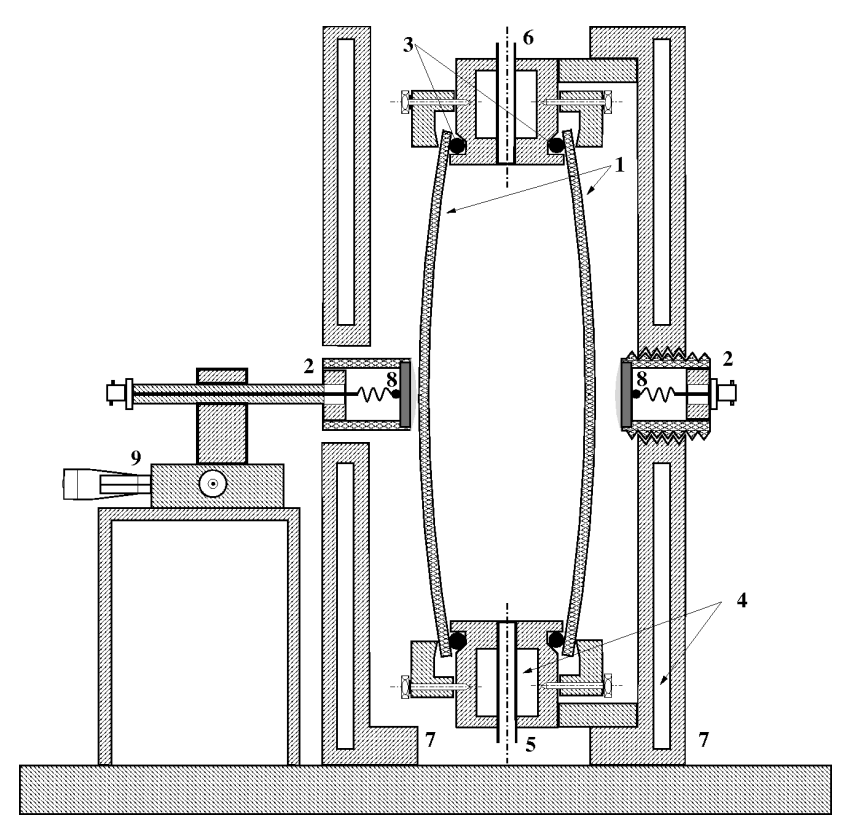

Abbildung 4.6: Querschnittszeichnung des Reflektorresonators. (1) Reflektoren; (2) Wandlerfassung; (3) O-Ringe; (4) Thermostatierkanäle; (5) Befüllkanal; (6) Belüftungskanal; (7) Grundplatte; (8) Wandler; (9) Verfahreinheit [92].

Im Gegensatz zu herkömmlichen Resonatoren, deren Messvolumen durch die Schallwandler abgeschlossen wird, wird die Zelle beim Reflektor-Resonator durch zwei konkave Rasierspiegel (Radius $R=60 \mathrm{~mm}$, Krümmungsradius $k=1 \mathrm{~m}$, Dicke $d=2 \mathrm{~mm}$ ) begrenzt. Der große Vorteil der Spiegel liegt darin, dass sie mit einer ausreichenden Präzision gefertigt werden, und sich gegenüber zahlreichen Materialien inert verhalten. Die Spiegel werden so in den Resonator eingesetzt, dass sie frei schwingen können, aber dennoch ortsfest eingespannt sind. Auf der Senderseite wird das Schallsignal zentral über den direkten Kontakt des Piezowandlers an den Spiegel gekoppelt, um eine gute Signalübertragung zu gewährleisten. Auf der Empfängerseite sind Reflektor und Wandler mechanisch voneinander getrennt, um zusätzliche Reflektorschwingungen von den zu messenden Resonanzen zu trennen. Eine Querschnittszeichnung des Reflektor-Resonators ist in Abb. 4.6 dargestellt $[92,93]$.

Mit dem Reflektor-Resonator sind Messungen zwischen $100 \mathrm{kHz}$ und $1 \mathrm{MHz}$ möglich. Er weist in diesem Bereich eine sehr hohe Güte auf; die daraus resultierenden geringen Halbwertsbreiten für schwach dämpfende Substanzen wie Wasser machten eine Weiterentwicklung des Messverfahrens nötig, um die Resonanzen mit genügend hoher Auflösung aufzunehmen. Der weiterentwickelte adaptive Scan-Algorithmus wird in Abschnitt 4.2.8 beschrieben.

\section{Der 8-MHz-Resonator}

Ein Anschluss an den Messbereich des Reflektor-Resonators wird durch den 8-MHzResonator mit vorgespannten Wandlerplatten nach Polacek [92] erreicht. Dieser Resonator deckt einen Frequenzbereich von $300 \mathrm{kHz}$ bis $24 \mathrm{MHz}$ ab. Die piezoelektrischen Wandler 
werden unter mechanischer Spannung in die Halterung eingesetzt, so dass sie eine konkave Form erhalten. Messungen zeigen, dass durch die Konkavität der Wandler in einem Frequenzbereich von $2 \mathrm{MHz}$ bis $5 \mathrm{MHz}$ die Güte des Resonators ansteigt [92]. Bei einen biplanaren Resonator wird bei hohen Frequenzen der Abstand der Radialmoden nach Gl. (4.18) immer geringer, weshalb sich die verschiedenen Moden nicht mehr auflösen lassen. Der bikonkave 8-MHz-Resonator kann zu höheren Frequenzen noch benutzt werden, da die Auflösung des äquidistanten Modenspektrums nach Gl. (4.19) insbesondere durch den Anstieg der Hintergrunddämpfung begrenzt wird.

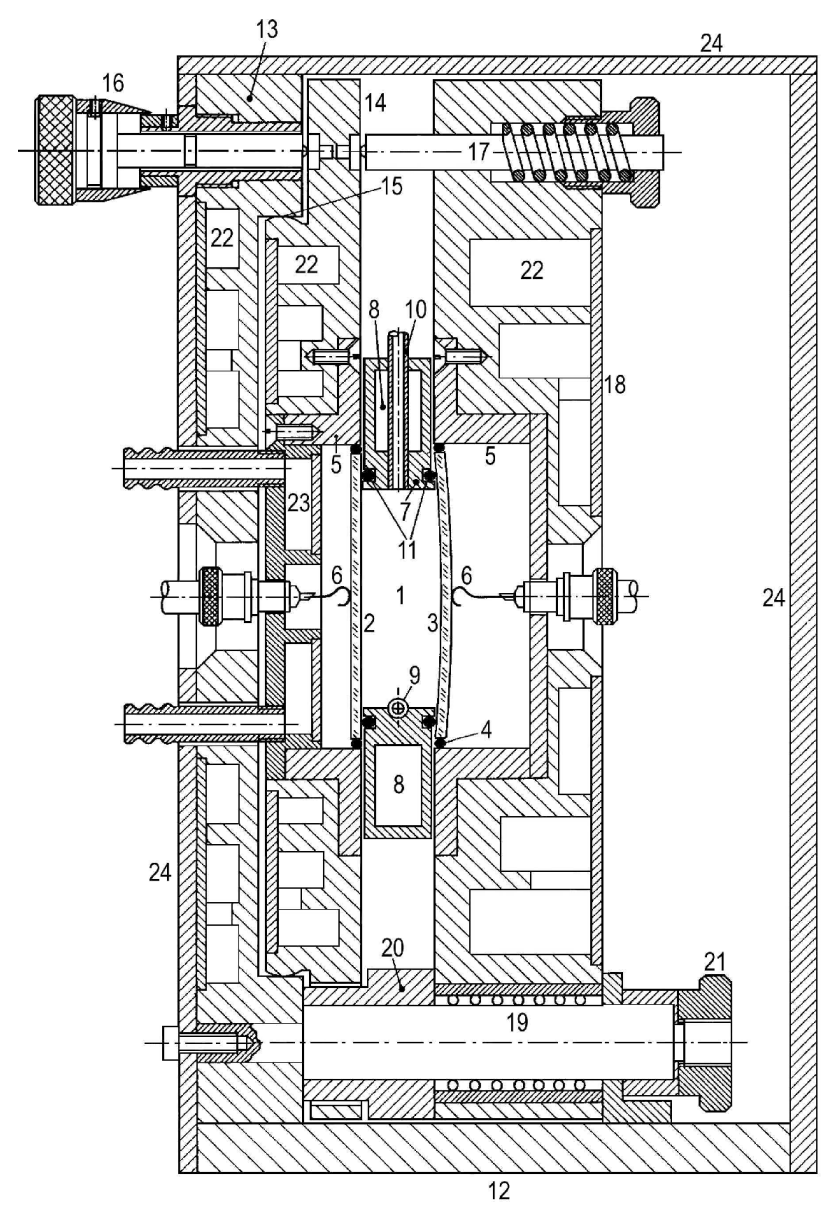

Abbildung 4.7: Querschnitt durch einen planar-konkaven Resonator. (1) Messvolumen; (2) planarer Wandler; (3) konkaver Wandler; (4) Einklebung mit elektrischer Kontaktierung zur Flüssigkeitsseite; (5) Quarzhalterung (V2A-Stahl); (6) rückwärtige Kontaktierung; (7) Zellberandung; (8),(22),(23) Thermostatierkanäle; (9) Einfüll- und (10) Belüftungskanäle; (11) Dichtungsringe; (12) Grundplatte; (13) Montageplatte der Senderseite; (14) verkippbarer Rahmen zur Paralleljustierung mittels (15) Kugelgelenk, (16) Feintriebschraube und (17) Druckfeder; (18) Montageplatte der Empfängerseite; (19) Kugelführung; (20) Abstandsstück zur Zelllängenfixierung; (21) Befestigungsschraube; (24) Schutzwände für die Temperaturabstimmung.

In Abb. 4.7 ist ein Querschnitt durch einen planar-konkaven Resonator dargestellt. Der 8-MHz-Resonator mit vorgespannten Wandlerplatten besitzt einen nahezu identischen Aufbau. 


\subsubsection{Ablauf der Messung}

Für die Bestimmung des Dämpfungsexponenten aus der Halbwertsbreite müssen nach Gl. (4.9) die Schallgeschwindigkeit in der Flüssigkeit und nach Gl. (4.15) die apparativen Verluste bekannt sein. Zur Bestimmung der Schallgeschwindigkeit aus Gl. (4.16) wird die Zelllänge $L$ benötigt. Die Zelllänge wird, ebenso wie die apparativen Verluste, durch eine Referenzmessung bestimmt. Gl. (4.16) liefert in diesem Fall bei bekannter Schallgeschwindigkeit die Zelllänge. Vor jeder Substanzmessung muss eine Referenzmessung durchgeführt werden, da die Zelllänge und die apparativen Eigenschaften empfindlich gegenüber äußeren Einflüssen sind.

Vor dem Befüllen des Resonators sind Messsubstanz und Resonator auf die Temperatur vorzuthermostatieren. Die Substanz wird langsam von unten in das Messvolumen über den Befüllkanal eingebracht. Dabei ist darauf zu achten, dass sich keine Luftblasen im Messvolumen bilden. Die Befüllung ist abgeschlossen, wenn die Substanz an der Oberseite aus dem Entlüftungskanal austritt.

Luftblasen im Messvolumen verfälschen die Messung des Absorptionskoeffizienten, da an der Grenzfläche Luft/Substanz die Schallwellen aus dem Messvolumen gestreut werden können. Durch die Mehrfachreflexion innerhalb des Resonators ist dieser Effekt die Hauptursache für fehlerhafte Messungen.

Ist die Substanz blasenfrei eingebracht, so wird der Resonator weiterhin bis zur endgültigen Messtemperatur thermostatiert. Für die letzten 1/10 Grad empfiehlt sich die "Verfolgung“ (Driftmessung, siehe Abb. B.3) einer schmalen Resonanz. Die Resonanzlage ist nach Gl. (4.7) von der Schallgeschwindigkeit und der Zelllänge abhängig. Da sich beide Parameter mit der Temperatur ändern, ist eine Verlagerung der Resonanz zu beobachten. Die Messung kann beginnen, wenn die Lage der Resonanz stabilisiert ist, und die Temperatur von der Endtemperatur um $\Delta T<0,02 \mathrm{~K}$ abweicht.

Die Messung der Resonanzen erfolgt mit einem Steuerprogramm, das Amplitude und Phase der Resonanz mit einer hohen Auflösung aufnimmt und speichert. An die Daten wird eine Lorentzkurve nach Gl. (4.8) angepasst, deren Mittenfrequenz und Halbwertsbreite separat gespeichert werden. Ein modernes Programm, das diese Aufgaben übernimmt, wird in Abschnitt 4.2.8 vorgestellt.

Eine genauere Anpassung der Messdaten wird nach der Messung vorgenommen. Dazu werden die Resonanzen manuell unter Berücksichtigung der Wandlergeometrie mit bis zu fünf Nebenmoden und einem Term für das elektrische Übersprechen nach

$$
T(f)=\left(\sum_{j=1}^{n} \frac{A_{j}}{\sinh \gamma_{j} L}+U_{\mathrm{el}} e^{i \phi_{\mathrm{el}}}\right) e^{i \phi_{0}}
$$


mit

$$
\begin{aligned}
f_{j} & : \text { Resonanzfrequenz der } j \text {-ten Nebenmode } \\
\gamma_{j}=\alpha_{j}+i \beta_{j} & : \text { komplexe Ausbreitungskonstante } \\
\phi_{0} & : \text { globale Phase } \\
L & : \text { Zelllänge } \\
U_{\mathrm{el}}, \phi_{\mathrm{el}} & : \text { Amplitude und Phase des elektrischen Übersprechens }
\end{aligned}
$$

unter Verwendung des Marquardt-Algorithmus [94] angepasst.

\subsubsection{Der Messplatz zur Messung der erweiterten Spektralfunktion S\#}

Ein zentraler Punkt dieser Arbeit ist die Messung der empirischen Skalierungsfunktion nach Bhattacharjee und Ferrell, die in Abschnitt 3.3 beschrieben wird. Der „Critical Slowing Down" nach Gl. (3.19) sagt eine Verlangsamung der kritischen Fluktuationen voraus, so dass diese im Wesentlichen einen Beitrag im niederfrequenten Bereich des Ultraschallabsorptionsspektrum liefern. Sind keine weiteren Beiträge durch z. B. Einzelzeitrelaxationen zu erwarten, so genügt es, die Skalierungsfunktion anhand von Absorptionsmessungen in einem sehr eng gewählten Messbereich zu bestimmen, für „einfache“ kritische Systeme wie in [9] kann die Skalierungsfunktion unter Verwendung eines Resonators im Frequenzbereich zwischen $0,1 \mathrm{MHz}$ und $1,5 \mathrm{MHz}$ bestimmt werden.

Im Falle der Lipidlösungen sind die kritischen Fluktuationen von zusätzlichen Einzelzeitrelaxationen überlagert [98, 100]. Um die einzelnen Beiträge trennen zu können, sind spektroskopische Untersuchungen in einem größeren Messbereich nötig. In [100] wird die erweiterte Spektralfunktion

$$
S^{\#}(\omega)=S(\omega)+\frac{A_{0}\left(\omega \tau_{0}\right)}{1+\left(\omega \tau_{0}\right)^{2}}
$$

mit

$$
\begin{aligned}
& S(\omega) \text { : Spektralfunktion } \\
& A_{0}, \tau_{0}: \text { Amplitude und Relaxationszeit des zusätzlichen Relaxationsterms }
\end{aligned}
$$

eingeführt, die die empirische Skalierungsfunktion um einen Debye-Spektralterm einer Einzelzeitrelaxation erweitert, die nur in der Nähe der kritischen Temperatur auftritt.

Der neue Messplatz zur Messung der erweiterten Spektralfunktion, dargestellt in Abb. 4.8, bietet die Möglichkeit, einen Frequenzbereich von $0,1 \mathrm{MHz}$ bis $24 \mathrm{MHz}$ abzudecken. Die grundlegende Idee hinter dem Aufbau ist, zwei Resonatoren $(4,5)$ gleichzeitig zu thermostatieren, um Fehler durch unterschiedliche Temperierungen auszuschließen. Besonders in der Nähe der kritischen Temperatur ändert sich die Absorption sehr stark mit der Temperatur, so dass bereits kleine Temperaturunterschiede zwischen den Resonatoren zu einem Versatz im Gesamtspektrum führen. 


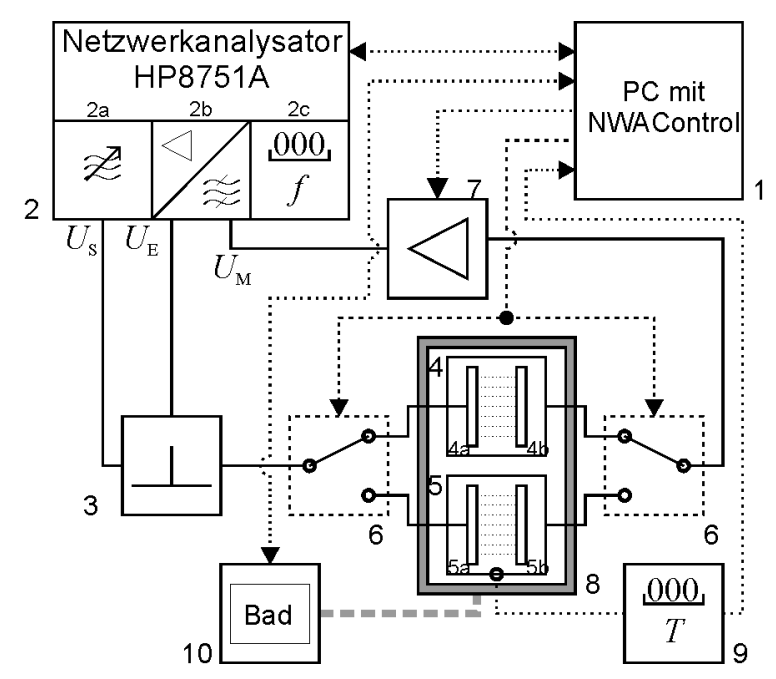

Abbildung 4.8: Blockschaltbild des neuen Messplatzes. Die durchgehende Linie symbolisiert den Weg des Messsignals, gepunktete Linien repräsentieren Mess- und Steuerleitungen, die gestrichelten Linien optionale Komponenten. (1) Steuerrechner; (2) Netzwerkanalysator HewlettPackard 8751A mit (2a) Generator, (2b) Zweikanal-Überlagerungsempfänger und (2c) Frequenzzähler; (3) Signalteiler; (4) 1-MHz-Reflektor-Resonator mit (4a) Sende- und (4b) Empfangswandler; (5) 8-MHz-Resonator mit (5a) Sende- und (5b)-Empfangswandler; (6) optionaler KoaxialSchalter; (7) regelbarer Verstärker mit paralleler Schnittstelle; (8) äußere Thermostatierhülle; (9) Digitalthermometer mit externem Pt100-Messfühler und RS232-Schnittstelle; (10) Lauda E200-Thermostat mit RS232-Schnittstelle.

Die verwendeten Resonatoren wurden bereits im vorhergehenden Abschnitt beschrieben. Sie sind an einen Thermostaten (Lauda E200) angeschlossen, dessen Temperatur über eine serielle Schnittstelle (RS232) durch einen Rechner (1) gesetzt werden kann. Das Wasser des Thermostaten durchströmt zuerst die Thermostatierkanäle des 1-MHz-Resonator (Abb. 4.6 (4)) und anschließend die des 8-MHz-Resonators (Abb. 4.7 (8, 22, 23)). Die Messaufbauten sind zusätzlich von einer thermostatierbaren Isolierhülle (Abb.4.8 (8)) umgeben, die für eine bessere Temperaturstabilität nötig ist.

Das elektrische Signal wird von einem Netzwerkanalysator (NWA) des Typs Hewlett Packard HP 8751A erzeugt (Abb. 4.8 (2)) und anschließend über einen Signalteiler (3) auf den Sendewandler eines Resonators $(4,5)$ gegeben. Die Umschaltung zwischen den Resonatoren erfolgt manuell, kann jedoch optional auch über Koaxialrelais (6) automatisch mit dem Steuerrechner (1) erfolgen. Das Signal des Empfangswandlers wird über einen regelbaren Verstärker (7) so verstärkt, dass die relative Signalamplitude $U_{\mathrm{M}} / U_{\mathrm{E}}$ zwischen $-25 \mathrm{~dB}$ und $-55 \mathrm{~dB}$ liegt. Für höhere Amplituden wird die Signalübertragung nichtlinear, die auszumessende Resonanz wird gestaucht, unterhalb -55 dB wird der Einfluss des Grundrauschens zu groß.

Das Auslesen und die Auswertung der Daten erfolgt mit dem Programm NWAControl, das Gegenstand des folgenden Abschnitts sein soll. Neben den Resonanzdaten wird zusätzlich die aktuelle Temperatur eingelesen, um bei späteren Auswertungen Fehler durch Temperaturschwankungen abschätzen zu können. 


\subsubsection{Das Programm NWAControl}

Die Bestimmung der Skalierungsfunktion setzt einen hohen Anspruch an Messgenauigkeit und Reproduzierbarkeit voraus. Dieses bedeutet zum einen, dass man eine „Kontrollinstanz" benötigt, die äußere Parameter wie die Temperatur $T$ überwacht, zum anderen muss gewährleistet sein, dass sehr schmale Resonanzen mit Halbwertsbreiten $\Delta f_{n}$ von wenigen Hertz, sowie sehr breite Resonanzen mit Halbwertsbreiten von einigen Kilohertz aufgenommen werden können. Erstere treten bei der Referenzmessung mit Wasser im 1MHz-Resonator auf, letztere sind vor allem für Frequenzen $f>10 \mathrm{MHz}$ in der Nähe der kritischen Temperatur für Lipidlösungen zu finden. Damit verknüpft ist ein großer Dynamikbereich des Resonatorausgangsignals von ungefähr $80 \mathrm{~dB}$, der an den Messbereich des Netzwerkanalysators angepasst werden muss.

All diese Aufgaben werden von dem Programm „NWAControl“ übernommen, das mittlerweile erfolgreich an allen Resonatormessplätzen eingesetzt wird.

\section{Ansteuerung der Messinstrumente}

An den Messplätzen werden sowohl kommerzielle Messinstrumente, als auch Geräte aus der Elektronikwerkstatt des III. Physikalischen Institutes (DPI) verwendet. Als Netzwerkanalysatoren werden der Hewlett Packard Network Analyzer 8751A und der Hewlett Packard Network \& Spectrum Analyzer 4951A verwendet, die über eine serielle IEEESchnittstellenkarte angesteuert werden. Die verwendeten IEEE-Schnittstellenkarten sind die interne PCI-Karte Keithley IEEE-488 und die externe IEEE-Schnittstelle Agilent 82357A, die an den USB-Ausgang des Rechners angeschlossen wird.

Da sich sowohl die Befehlssätze der NWAs, als auch die der Schnittstellenkarten untereinander deutlich unterschieden, ist für die Geräte ein einheitlicher Befehlssatz in NWAControl implementiert worden, über den eine Ansteuerung möglich ist. Über diese Programmierschnittstelle ist es leicht möglich, auch andere Analysatoren und IEEE-Karten mit NWAControl anzusteuern. Man spricht bei diesem Verfahren auch von der „Kapselung“ von Programmteilen.

Ebenfalls gekapselt sind die Ansteuerung von Digitalthermometer, Thermostat und Verstärker. Das Thermometer mit thermisch stabilisierter Elektronik, das aus der Elektronikwerkstatt des DPI stammt, und der Thermostat Lauda E200 werden an den seriellen Schnittstellen des Computers betrieben.

Der regelbare Verstärker aus der Elektronikwerkstatt besitzt neben den Verstärkerstufen 10, 20, 30, 40 dB auch die Möglichkeit, das Signal um 20 dB zu dämpfen. Dieses ist mitunter in der Nähe der Quarzresonanz notwendig, wo der Resonator nach Gl. (4.17) eine hohe Sensitivität besitzt. Der Verstärker wird über die parallele Schnittstelle angesteuert.

Alle Geräte, für die ein gekapselter Befehlssatz besteht, sind an den Messplätzen beliebig austauschbar. Die aktuelle Konfiguration wird aus einer Textdatei, die auf der Festplatte des Rechners liegt, eingelesen. 


\section{Der adaptive Scan-Algorithmus}

Um die Zellresonanzen einzulesen (zu „scannen“), wird zuerst ein breitbandiges Spektrum des Resonators aufgenommen, anhand dessen die zu scannenden Resonanzen ausgewählt werden. Dabei wird der Experimentator durch das Programm unterstützt, indem der Rechner bei Kenntnis des Resonanzabstandes $f_{n}-f_{n-1}$ die nächste Resonanz im Auswahlbereich zentriert. Die Auswahl der Hauptmode wird somit beim Auftreten mehrerer Nebenmoden erleichtert. Die Resonanzen werden per Mausklick ausgewählt. Je nach scheinbarem Einfluss der Nebenmoden kann der Anwender zusätzlich mehrere Nebenmoden zum Scannen auswählen. Dieses ist insbesondere hilfreich bei stark dämpfenden Substanzen. Bildschirmfotos von NWAControl sind im Anhang zu finden. Nach Auswahl der Resonanzen werden die selektierten Frequenzbereiche an den adaptiven Scan-Algorithmus übergeben.

Der adaptive Scan-Algorithmus ist das Herzstück von NWAControl. Er regelt die Anpassung der Scanbereichsbreite (Span), der Signalverstärkung und der Bandbreite der Zwischenfrequenz. Während die Bedeutung von Span und Signalverstärkung sofort zugänglich ist, bedarf es bei der Bandbreite der Zwischenfrequenz einer kurzen Erklärung.

In einem NWA wird das Eingangssignal, das sich über einen großen Frequenzbereich erstreckt mittels eines durchstimmbaren lokalen Oszillators (LO) in mehreren Stufen auf eine feste Zwischenfrequenz $\Delta f_{\mathrm{IF}}$ gemischt. Der Mischungsprozess ist nichtlinear, es entstehen mehrere Seitenbänder höherer Ordnung. Die entstehenden Zwischenfrequenzen werden mit einem Gaußfilter der Breite $\Delta f_{\mathrm{IF}}\left(\mathrm{IFBW}^{1}\right)$ gefaltet. Das Ergebnis dieser Faltung wird als Amplitudeninformation ausgegeben. Je schmaler der Gaußfilter ist, desto besser kann das ursprüngliche Signal aufgelöst werden. Die Verringerung von $\Delta f_{\mathrm{IF}}$ führt aber zu einer Erhöhung der Scan-Dauer [1]

$$
\tau_{\text {scan }} \sim \frac{1}{\left(\Delta f_{\mathrm{IF}}\right)^{2}}
$$

Um einen schnellen Ablauf der Messung zu gewährleisten, wird die IFBW an die Breite der Resonanz angepasst. Empirisch ergibt sich eine Abschätzung für die Zwischenfrequenz

$$
\Delta f_{\mathrm{IF}}<\zeta \cdot \Delta f \quad \operatorname{mit} \zeta=\frac{2}{3}
$$

Der adaptive Scan-Algorithmus überprüft zuerst, ob in dem zuvor manuell ausgewählten Frequenzbereich (Span) eine Resonanz gefunden werden kann. Ist dieses nicht der Fall, so wird die Breite des Spans verdoppelt und die Resonanz erneut gesucht. Durch Temperaturänderung kann es vorkommen, dass bei schmalen Frequenzbereichen die Resonanz aus dem ausgewählten Bereich heraus wandert. Durch die sukzessive Vergrößerung des Spans werden die Resonanzen wieder gefunden. Die Suche ist beendet, wenn eine Resonanz gefunden wurde, oder der Span größer als der Abstand der Zellresonanzen ist, denn in dem letzten Fall muss theoretisch eine Resonanz vorliegen. Tritt dies nicht ein, so liegt ein technisches oder physikalisches Problem vor.

\footnotetext{
${ }^{1}$ Je nachdem, ob für die Realisierung dieses Filters ein (1) analoger oder (2) digitaler Filter eingesetzt wird, spricht man von (1) Interfrequency Bandwidth oder (2) Resolution Bandwidth [1]. Es wird im Folgenden nur der Begriff IFBW verwendet.
} 


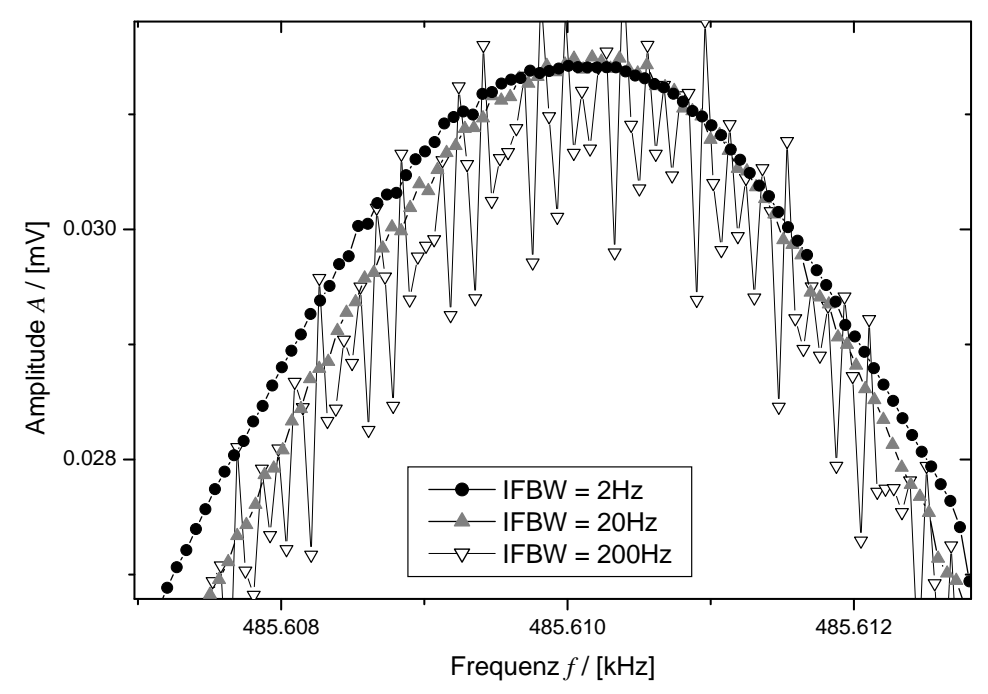

Abbildung 4.9: Einfluss der IFBW auf die Form der Resonanzkurve. Die Resonanz besitzt eine Halbwertsbreite von $\Delta f \approx 8 \mathrm{~Hz}$. Man erkennt deutlich den Einfluss eines schnellen Scannes mit hohen Werten für die IFBW. Für schmalere Resonanzen wird die Form der Kurve zunehmend unsymmetrisch für große IFBW.

Das Finden einer Resonanz bedeutet in diesem Zusammenhang, dass eine Amplitudenspitze innerhalb des Frequenzbereiches durch eine Lorentzkurve nach Gl. (4.8) beschrieben werden kann. Die Anpassung der Daten erfolgt mit dem Marquardt-Algorithmus [94], der als Lösung Amplitude $A_{\text {Lor }}$, Mittenfrequenz $f_{\text {Lor }}$ und Halbwertsbreite $\Delta f_{\text {Lor }}$ der Resonanz liefert. Der Erfolg einer Anpassung hängt entscheidend von den Startparametern ab. Um diese zu bestimmen, werden drei Kriterien überprüft:

1. Gibt es Spitzen (über mehrere Datenpunkte), die einen 3dB-Abfall aufweisen? Falls ja, dann werden die maximale Amplitude, die dazugehörige (Mitten)frequenz und die Breite der Spitze am 3dB-Abfall als Startparameter verwendet. Wenn nicht, dann

2. werden ausgeprägte Spitzen gesucht, die sich über mehrere Datenpunkte erstrecken. Falls solche Spitzen existieren, dann werden wiederum deren maximale Amplituden und die dazugehörige Frequenzen übernommen. Als Halbwertsbreite wird 1\% des vorgegebenen Messbereiches angenommen.

3. Sollte diese Suche auch kein Ergebnis bringen, dann wird das Maximum und dessen Frequenz, sowie 1\% des Messbereiches als Startparameter angenommen. Dieser Fall dient lediglich als „Rettungsanker“ für den Algorithmus.

Scheitert der Marquardt-Algorithmus mit diesen Startparametern, so werden die freien Parameter abwechselnd festgehalten. Durch die verschiedenen Methoden die Parameter zu bestimmen, wird für nahezu jede Resonanz eine gute Anpassung der Hauptmode gefunden. Probleme treten bei sehr schwachen Resonanzen auf, die trotz Verstärkung eine maximale Amplitude von weniger als $-65 \mathrm{~dB}$ aufweisen, und bei hochfrequenten Resonanzen, deren Nebenmaxima nicht mehr sauber getrennt werden können. 

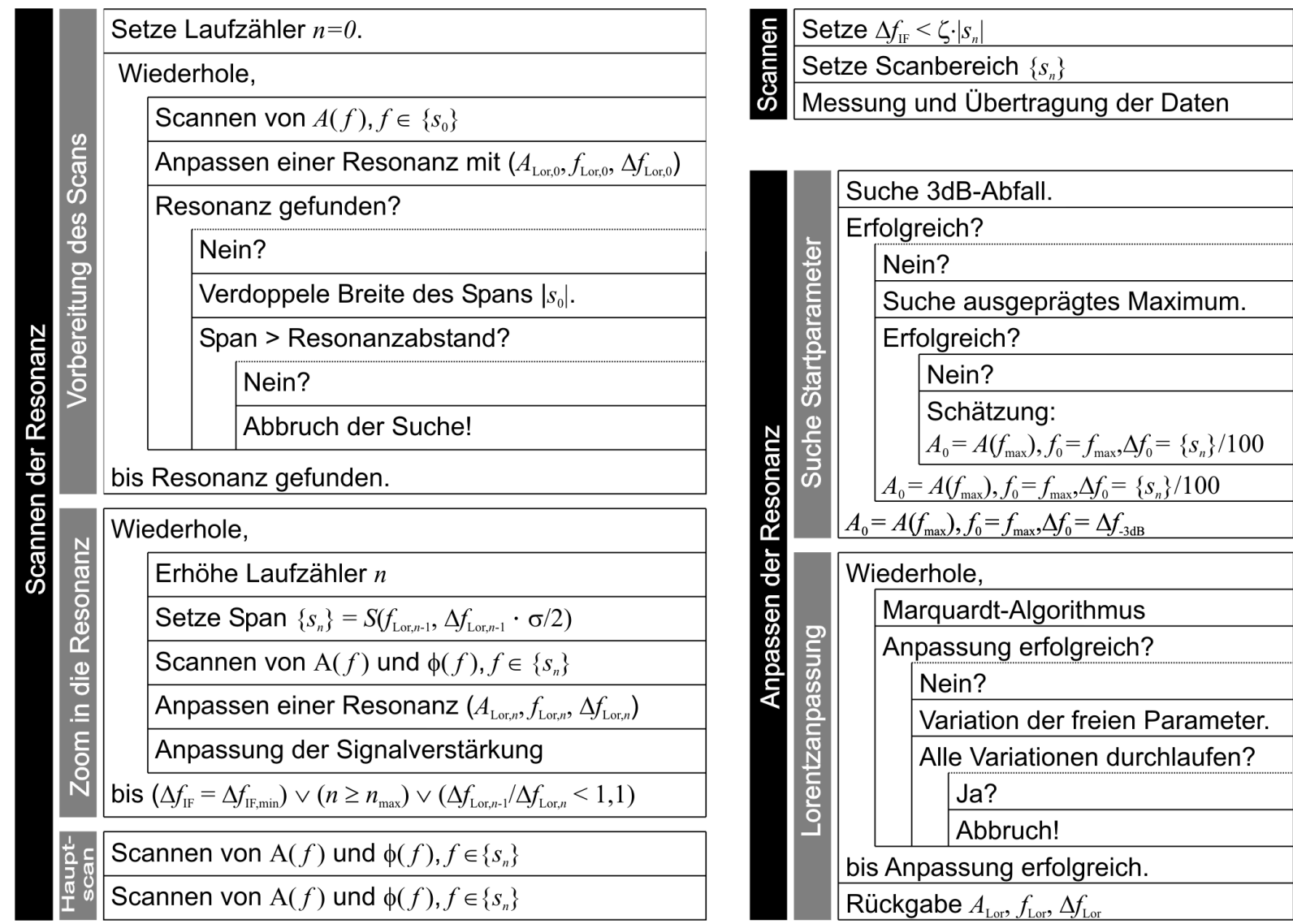

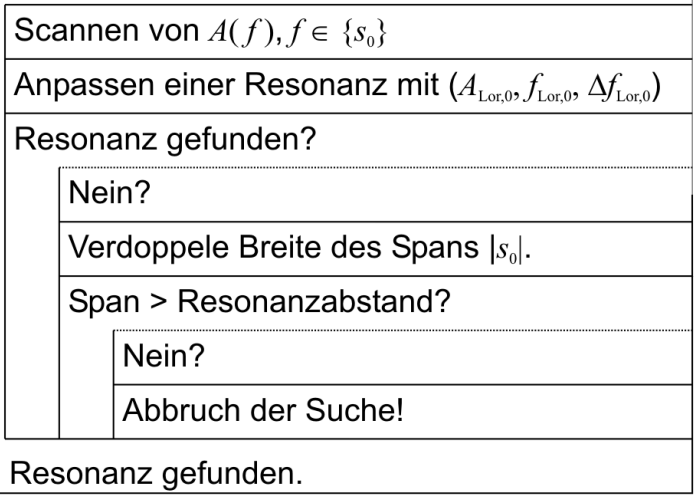

Wiederhole,

Erhöhe Laufzähler $n$

Setze Span $\left\{s_{n}\right\}=S\left(f_{\text {Lor, } n-1-1}, \Delta f_{\text {Lor }, n-1} \cdot \sigma / 2\right)$

Scannen von $\mathrm{A}(f)$ und $\phi(f), f \in\left\{s_{n}\right\}$

Anpassen einer Resonanz $\left(A_{\mathrm{Lor}, n}, f_{\mathrm{Lor}, n}, \Delta f_{\mathrm{Lor}, n}\right)$

Anpassung der Signalverstärkung

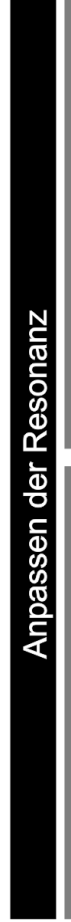

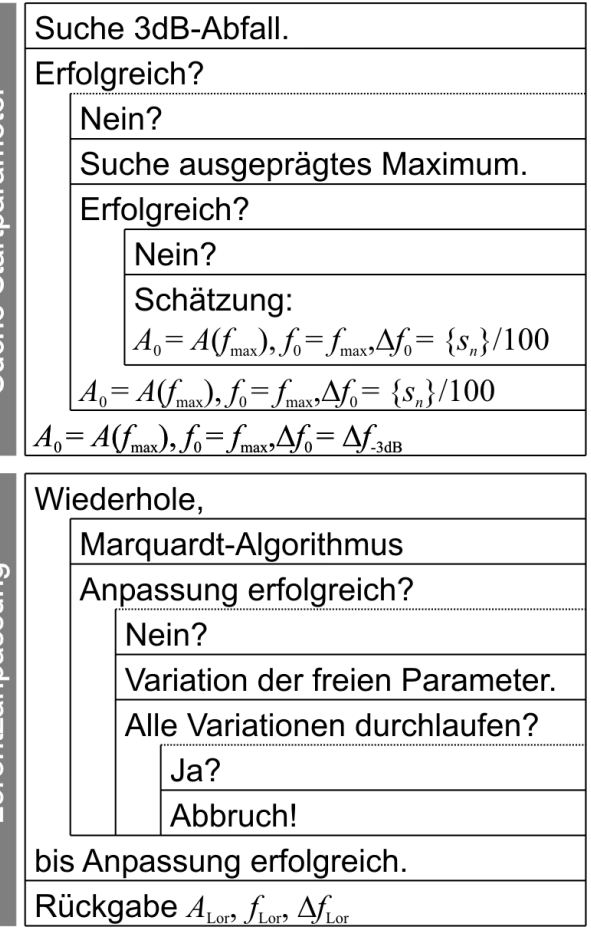

$n$ : Zähler für die verschiedenen Scan-Durchgänge

$n_{\max }:$ Maximale Anzahl von Scan-Durchgängen

$\left\{s_{n}\right\}$ : Frequenzbereich (Span) des $n$-ten Scan-Durchgangs

$S(f, w)$ : „Hilfsnotation“ zur Erzeugung eines Frequenzbereiches

$[f-w, f+w]$

$\left|s_{n}\right| \quad: \quad$ Breite des $n$-ten Spans

$\sigma$ : Faktor zur Anpassung der Span-Breite

$A(f), \phi(f) \quad$ : Amplitude und Phase bei der Frequenz $f$

$A_{\text {Lor }}, f_{\text {Lor }}, \Delta f_{\text {Lor }}:$ Amplitude, Mittenfrequenz und Halbwertsbreite der

Lorentzkurve

$\Delta f_{\mathrm{IF}}:$ Halbwertsbreite der Zwischenfrequenz (IFBW)

$\Delta f_{\mathrm{IF}, \text { min }}:$ kleinste einstellbare IFBW

$\zeta$ : Bandbreitenfaktor zur Anpassung der IFBW

Abbildung 4.10: Ablaufdiagramm des adaptiven Scan-Algorithmus. Zu Beginn wird der Scan vorbereitet, indem geprüft wird, ob in dem vorgegebenen Suchbereich eine Resonanz vorhanden ist: wenn nicht, wird der Suchbereich erweitert. Im nächsten Programmteil wird schrittweise in den Resonanzbereich „gezoomt“. Dazu wird kontinuierlich die Halbwertsbreite der Resonanz anhand der Amplitudeninformation bestimmt und der Frequenzbereich (Span) und die Zwischenfrequenz angepasst. Abschließend wird in dem zuletzt eingestellten Frequenzbereich sowohl Amplitude, als auch Phase aufgenommen. Ebenfalls aufgenommen werden auch Amplitudenund Phaseninformation des zuvor manuell ausgewählten Frequenzbereiches. 
Wird eine Resonanz nach diesem Verfahren gefunden, so wird in die Resonanz „gezoomt“. Die Anpassung der Daten mittels einer Lorentzkurve ist nur gültig für kleine Abweichungen von der Mittenfrequenz. Dabei werden nach jedem Scan die Daten mit einer Lorentzkurve angepasst, und der Frequenzbereich als Vielfaches der Halbwertsbreite $\sigma \cdot \Delta f_{\text {Lor }}$ ausgewählt (Siehe auch Abb. B.2). Diese Iteration ist beendet, wenn sich die Halbwertsbreite um weniger als $10 \%$ verringert; d. h. wenn

$$
\frac{\Delta f_{\text {Lor }, n}}{\Delta f_{\text {Lor }, n-1}}<1,1
$$

oder wenn eine maximale Anzahl von Scans erreicht wurde $\left(n=n_{\max }\right)$, oder die IFBW ihren kleinsten einstellbaren Wert $\left(\Delta f_{\mathrm{IF}}=\Delta f_{\mathrm{IF}, \mathrm{min}}\right)$ erreicht hat, der von dem verwendeten Netzwerkanalysator abhängt.

Nachdem über diesen Algorithmus der bestmögliche Frequenzbereich und die optimale IFBW ausgewählt ist, wird abschließend die Amplitude $A(f)$ und die Phase $\phi(f)$ in diesem Frequenzintervall gemessen. Zusätzlich wird noch die Amplituden- und Phaseninformation des manuell gewählten Spans ermittelt. Dieses ist hilfreich, und oft auch notwendig, wenn die Hauptamplitude durch mehrere Nebenmoden beeinflusst wird, oder wenn der Suchalgorithmus scheitert (siehe Abb. B.2).

Probleme mit dem Algorithmus wurden in den Messungen im Rahmen dieser Arbeit nur bei Frequenzen des 1-MHz-Resonators unterhalb $250 \mathrm{kHz}$ für starke Dämpfungen festgestellt. Der Algorithmus zoomt in diesem Falle gelegentlich in zufällige Amplitudenspitzen des Rauschens hinein. Bei hohen Frequenzen des 8-MHz-Resonators tritt in der Nähe der Scherresonanz des Quarzwandlers das Phänomen auf, dass die erste Nebenmode eine höhere Amplitude besitzt als die Hauptmode. Hier kann der Suchalgorithmus ggf. die Nebenresonanz auswählen. Durch die zusätzliche Messung des zuvor manuell ausgewählten Messbereiches können aber auch solche Resonanzen noch ausgewertet werden.

Die Messdauer des Algorithmus hängt stark von der IFBW bzw. der Resonanzbreite ab. Der Netzwerkanalysator HP8751A benötigt für eine Messung mit $\Delta f_{\mathrm{IF}}=20 \mathrm{~Hz}$ eine Zeit von ungefähr $80 \mathrm{~s}$, wobei der letzte Scan, der Amplituden- und Phaseninformation enthält, ungefähr $20 \mathrm{~s}$ andauert. Bei der Messung mit $\Delta f_{\mathrm{IF}}=2 \mathrm{~Hz}$ benötigt der Scan von Amplitude und Phase schon schon über 120 s. Die Gesamtmessdauer des Algorithmus beläuft sich auf über 200 s.

Die meisten der Parameter für den adaptiven Scan-Algorithmus können frei vorgegeben werden, so dass die Suche sehr flexibel an die jeweiligen Gegebenheiten angepasst werden kann.

\subsubsection{Fehlerabschätzung}

Die Messung des Absorptionsspektrums mit Hilfe des Resonatorverfahrens wird durch verschiedene Fehlerquellen beeinflusst, die im folgenden erläutert werden sollen.

Fehlerhafte Befüllung. Wie schon in Abschnitt 4.2.6 beschrieben, ist eine Befüllung des Resonators ohne Luftblasen unerlässlich. Luftblasen führen zu einer Streuung der Schallwelle und damit zu einer Zusatzabsorption und Verbreiterung der Resonanzbreiten. Häufig 
ist eine fehlerhafte Befüllung durch Vergleich mit den Resonanzbreiten anderer Messungen zu erkennen. Mitunter treten auch zusätzliche Nebenmoden auf, die dadurch entstehen, dass die Schallwelle bereits nach Durchlaufen einer kürzeren Wegstrecke reflektiert wird.

Ist der Anschluss der Resonatormessdaten an den höherfrequenten Bereich des Absorptionsspektrums nicht möglich, so ist die Resonatormessung mit Referenz- und Substanzmessung zu wiederholen.

Temperaturfehler. Die Resonanzfrequenz und Halbwertsbreite der Resonanz werden stark von der Umgebungstemperatur beeinflusst. Insbesondere für die in dieser Arbeit untersuchten kritische Systeme ist die Temperatur aufgrund der Abhängigkeit thermodynamischer Parameter nach einem Potenzgesetz sehr genau einzuhalten. Für die Untersuchungen wurden die Temperaturschwankungen durch lange Thermostatierung auf $\Delta T<0,02 \mathrm{~K}$ stabilisiert.

Fehler in der Referenzmessung. Die zuvor beschriebenen Messfehler treffen sowohl auf die Substanz- als auch auf die Referenzmessung zu. Der prozentuale Fehler des Messergebnisses erhöht sich nach Gl. (4.15) dann, wenn die Dämpfung von Referenz und Substanz ähnlich sind. Bei DMPC/Wasser-Systemen werden diese Fehler für Temperaturen weit weg vom Phasenübergang wichtig.

Fehler bei der Anpassung. Die Anpassung der Resonanzen nach Gl. (4.22) birgt bei hohen Frequenzen des 8-MHz-Resonators systematische Fehler, da hier insbesondere für das Lipidsystem in der Nähe der kritischen Temperatur die Dämpfung sehr hoch ist und die Nebenmoden der Zellresonanzen miteinander verschmelzen und nicht mehr sauber getrennt werden können. Der 1-MHz-Resonator hingegen weist über den gesamten Messbereich sehr schmale und ausgeprägte Resonanzen auf, deren Nebenmoden leicht voneinander getrennt werden können.

Nach [92, 98] und eigenen Abschätzungen aus Mehrfachmessungen und dem Überlapp von Messbereichen verschiedener Messapparaturen können für die Resonatoren die Fehler in Tab. 4.1 angegeben werden.

Tabelle 4.1: Fehler des Resonatorverfahrens. Die angegebenen Werte können anhand eigener Messdaten und nach Messungen von [92, 93, 98] abgeschätzt werden.

\begin{tabular}{|c|c|c|c|}
\hline Resonator & Messintervall & Absorption $\left(\alpha / f^{2}\right)$ & Fehler $\Delta \alpha / \alpha$ \\
\hline \hline 1-MHz-Reflektor- & $0,1-0,3 \mathrm{MHz}$ & $\left(\alpha / f^{2}\right)<50 \cdot 10^{-15} \mathrm{~s}^{2} / \mathrm{m}$ & $10 \%$ \\
Resonator & $0,1-0,3 \mathrm{MHz}$ & $\left(\alpha / f^{2}\right) \geq 50 \cdot 10^{-15} \mathrm{~s}^{2} / \mathrm{m}$ & $5 \%$ \\
& $0,3-0,9 \mathrm{MHz}$ & & $5 \%$ \\
\hline 8-MHz-Resonator & $0,3-1,5 \mathrm{MHz}$ & & $10 \%$ \\
& $1,5-7,3 \mathrm{MHz}$ & & $5 \%$ \\
& $8,5-12,0 \mathrm{MHz}$ & $\left(\alpha / f^{2}\right)<50 \cdot 10^{-15} \mathrm{~s}^{2} / \mathrm{m}$ & $5 \%$ \\
& $8,5-12,0 \mathrm{MHz}$ & $\left(\alpha / f^{2}\right) \geq 50 \cdot 10^{-15} \mathrm{~s}^{2} / \mathrm{m}$ & $10 \%$ \\
\hline
\end{tabular}




\subsection{Das Schwingungspulstransmissionsverfahren}

\subsubsection{Das Messprinzip}

In der Messzelle des Schwingungspulstransmissionsverfahrens, kurz „Pulszelle“, befinden sich zwei parallele Piezowandler im Abstand $x$ zueinander. Der Raum zwischen den Wandlern ist vollständig mit der Probe gefüllt.

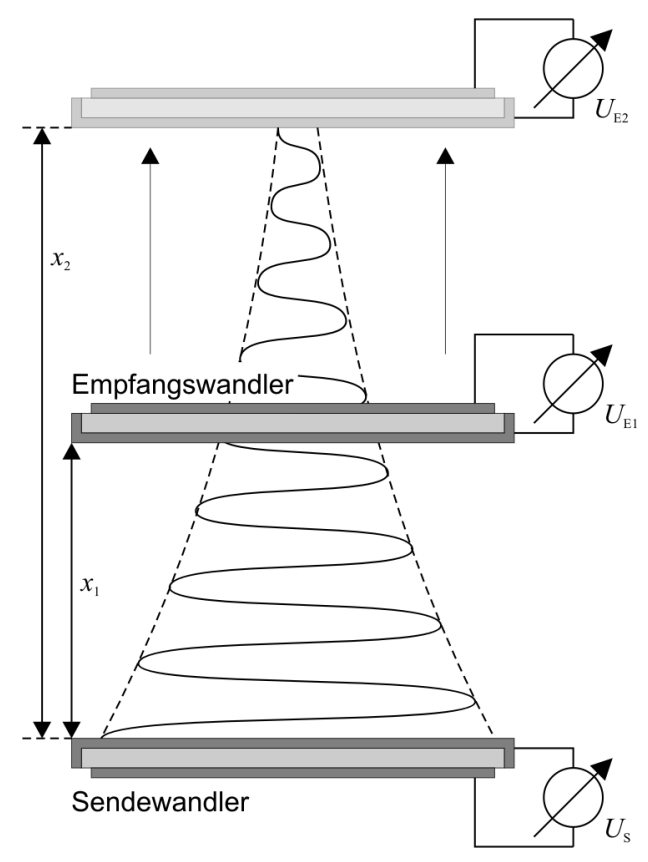

Abbildung 4.11: Schematische Darstellung des Messprinzips des Schwingungspulstransmissionsverfahrens. Die vom Sendewandler abgestrahlte Schallwelle wird bei verschiedenen Abständen $x_{i}$ durch einen Empfangswandler wieder in ein elektrisches Signal umgewandelt.

Während der Messung wird der Empfangswandler kontinuierlich gegenüber dem ortsfesten Sendewandler bewegt, und die Schalldruckamplitude in Abhängigkeit vom Ort gemessen, wie in Abb. 4.11 skizziert. Nach Gl. (3.1) ist ein exponentieller Abfall der Schalldruckamplitude zu erwarten.

Die tatsächliche Schalldruckamplitude ergibt sich aus der Berücksichtigung der Mehrfachreflexion an den Wandlern. Wie bereits für den idealen Resonator in Abschnitt 4.2.1 hergeleitet, erhält man unter Berücksichtung von Mehrfachreflexionen mit $0<r<1$ als Schalldruckamplitude am Empfangswandler

$$
p_{\mathrm{e}}=p_{\mathrm{s}} \frac{(1+r) e^{-\gamma x} e^{i \omega t}}{1-r^{2} e^{-2 \gamma x}}
$$

mit

$p_{\mathrm{s}, \mathrm{e}}:$ Schalldruckamplitude an Sende- und Empfangswandler

$x:$ Abstand der Wandler

$\gamma=\alpha+i \beta:$ Komplexe Ausbreitungskonstante

$r$ : Reflexionskoeffizient. 
Die meist symmetrischen Wandlerzuleitungen wirken ebenfalls als Sende- und Empfangsantennen für elektromagnetische Felder. Der Beitrag dieser Felder fließt als elektrisches Übersprechen mit einer Amplitude $A_{\mathrm{el}}$ und einer Phase $\phi_{\mathrm{el}}$ in die Übertragungsfunktion ein. Das elektrische Übersprechen kann bei niedrigen Frequenzen vernachlässigt werden, so dass Gl. (4.26) angenommen werden kann:

$$
\begin{aligned}
p_{\mathrm{e}} & =p_{\mathrm{s}} \frac{(1+r) e^{-\gamma x} e^{i \omega t}}{1-r^{2} e^{-2 \gamma x}}+A_{\mathrm{el}} e^{i \phi_{\mathrm{el}}} \\
& \approx p_{\mathrm{s}} \frac{(1+r) e^{-\alpha x}}{\sqrt{1+r^{4} e^{-4 \alpha x}+2 r^{2} e^{-2 \alpha x} \cos (2 \beta x)}} .
\end{aligned}
$$

Die Überlagerung der reflektierten Schallwellen führt zu der so genannten $(\lambda / 2)$-Welligkeit bei kleinen Abständen. Aus der Bestimmung der Amplitudenmaxima kann so auch die Schallgeschwindigkeit der Probe ermittelt werden. Aufgrund des schnellen Abklingens der Exponentialfunktionen im Nenner des Quotienten reduziert sich für Startabstände $x_{\mathrm{s}}>3 / \alpha$ die Abhängigkeit der Schalldruckamplitude $p_{\mathrm{e}}$ auf ein einfaches Exponentialgesetz

$$
p_{\mathrm{e}}(x)=p_{\mathrm{s}} e^{-\alpha x}
$$

das nach Gl. (3.1) erwartet wird.

Durch die Abstrahlung von Schwingungspulsen können die reflektierten Signale zeitlich voneinander getrennt werden, wenn gilt

$$
x>c_{\mathrm{s}} \tau_{\text {puls }},
$$

wobei $\tau_{\text {puls }}$ die Länge eines Pulses ist. Auf diesem Wege kann auch die akustische von der elektromagnetischen Signalübertragung unterschieden werden.

Bei hohen Frequenzen, bei denen aufgrund der hohen Hintergrundabsorption nur kurze Fahrwege möglich sind, werden die akustischen Signale durch den Einsatz von Verzögerungsleitungen aus Quarz oder Saphir voneinander und vom elektrischen Übersprechen getrennt.

Die Anwendbarkeit des Schwingungspulstransmissionsverfahrens wird durch die Verringerung der klassischen Dämpfung zu kleinen Frequenzen gemäß Gl. (3.7) begrenzt. Für Wasser mit $\left(\alpha / f^{2}\right)=21,28 \cdot 10^{-15} \mathrm{~s}^{2} / \mathrm{m}$ bei $25^{\circ} \mathrm{C}$ fällt die Schalldruckamplitude bei $f=10 \mathrm{MHz}$ nach

$$
p_{-3 \mathrm{~dB}}=\frac{p_{0}}{2}=p_{0} \cdot e^{-\alpha x} \Rightarrow x=\frac{\ln 2}{\left(21,28 \cdot 10^{-15}\right) \cdot 10^{14}} \approx 32,6 \mathrm{~cm}
$$

um $3 \mathrm{~dB}$ ab, bei $f=1 \mathrm{MHz}$ beträgt dieser Wert bereits $x=32,6 \mathrm{~m}$. Aufgrund der technischen Schwierigkeiten, die mit diesen langen Fahrwegen verbunden sind, liegt die Untergrenze der Anwendbarkeit dieses Verfahrens bei $f_{\text {lim }}=10-15 \mathrm{MHz}$.

\subsubsection{Der Messplatz für das Schwingungspulstransmissionsverfahren}

In Abb. 4.12 ist das Blockschaltbild für die Pulszellenmessplätze dargestellt. Im Synthetisiersender (1) wird ein harmonisches, elektrisches Signal bei einer Ausgangsleistung von 
$-10 \mathrm{dBm}$ bis $-5 \mathrm{dBm}$ (entsprechend $0,1 \mathrm{~mW}$ bis $0,3 \mathrm{~mW}$ ) erzeugt. Dieses Signal wird durch den PIN-Diodenschalter (2) mit einem Triggerpuls (3) von ungefähr $1 \mu$ s gemischt und anschließend mit dem Verstärker (4) um maximal 40dB verstärkt.

Über die Koaxialrelais (5), die über eine Relaissteuerung (24) mit dem Steuerrechner (25) geschaltet werden, wird das Signal in den Mess- oder Referenzzweig geleitet. Ein variables Dämpfungsglied (6) ermöglicht, die Signalamplitude in Mess- und Referenzzweig anzugleichen.

Der piezoelektrische Sendekristall (8a) wandelt, wie in Abschnitt 4.3.1 beschrieben, das pulsmodulierte elektrische Signal in eine mechanische Schwingung um. Der Empfangswandler (8b) kann über einen Schrittmotor (11) - bzw. Piezotranslator bei der PZTZelle - mittels der Ansteuerung (21) parallel zum Sendewandler bewegt werden. Bei der 10-MHz-Pulszelle und der PZT-Zelle können Reflexionsverluste durch längenvariable Kurzschlussstichleitungen durch Reduktion des Impedanzsprungs am Empfangswandler vermindert werden.

Im Referenzzweig wird das Signal durch ein Cut-Off-Dämpfungsglied (9) geführt. Das Cut-Off-Dämpfungsglied besteht aus zwei axial mit Schrittmotoren gegeneinander verschiebbaren Koaxialleitern. Die Koaxialleiter werden unterhalb der Grenzfrequenz ihrer Grundmode betrieben. Zwischen den verschiebbaren Innenleitern breitet sich daher das elektrische Signal als evaneszentes Feld mit einem exponentiellen Abfall aus. Auf diese Weise kann die Kennlinie des Messaufbaus bei verschiedenen Dämpfungen aufgenommen werden. Die Dämpfungsglieder (10) reduzieren die durch Reflexionen entstehende Welligkeit des Signals.

Die Signale aus Referenz- und Messzweig werden von einem Überlagerungempfänger (1316) gemessen. Durch eine vom Rechner (25) getriggerte (20) Sample-and-Hold-Schaltung (18) wird das Signal in den Rechner eingelesen.

Aus der Kennlinie des Systems, die mit Hilfe des Referenzsignals gewonnen wird, kann nun die ortsabhängige (22) Signalamplitude bestimmt und daraus der Dämpfungsexponent $\alpha$ ermittelt werden.

Die wesentlichen Eigenschaften der Pulszellen sind in Tab. 4.2 zusammen gefasst.

\subsubsection{Ablauf der Messung}

Vor der Messung sind die Messzellen gründlich mit Methanol oder einem anderen geeigneten Lösungsmittel zu reinigen und mit Stickstoff zu trocknen. Im nächsten Schritt wird das Messvolumen mit der entgasten Probensubstanz langsam befüllt, so dass sich keine Blasen in der Flüssigkeit oder an der Flüssigkeitsoberfläche bilden.

Nach dem Befüllen sind die Messzelle und die Probensubstanz auf die Messtemperatur zu thermostatieren. Dieser Vorgang beschleunigt sich, wenn Messzelle und Probensubstanz bereits vorthermostatiert sind. Kurz vor dem Erreichen der Endtemperatur wird eine Nullpunktseichung des Wandlerabstandes durchgeführt.

Dazu wird ein Signal geeigneter Frequenz und Amplitude, abhängig von den Wandlereigenschaften, am Synthetisiersender eingestellt und der Überlagerungsempfänger so eingestellt, dass das empfangene Signal maximal wird. 


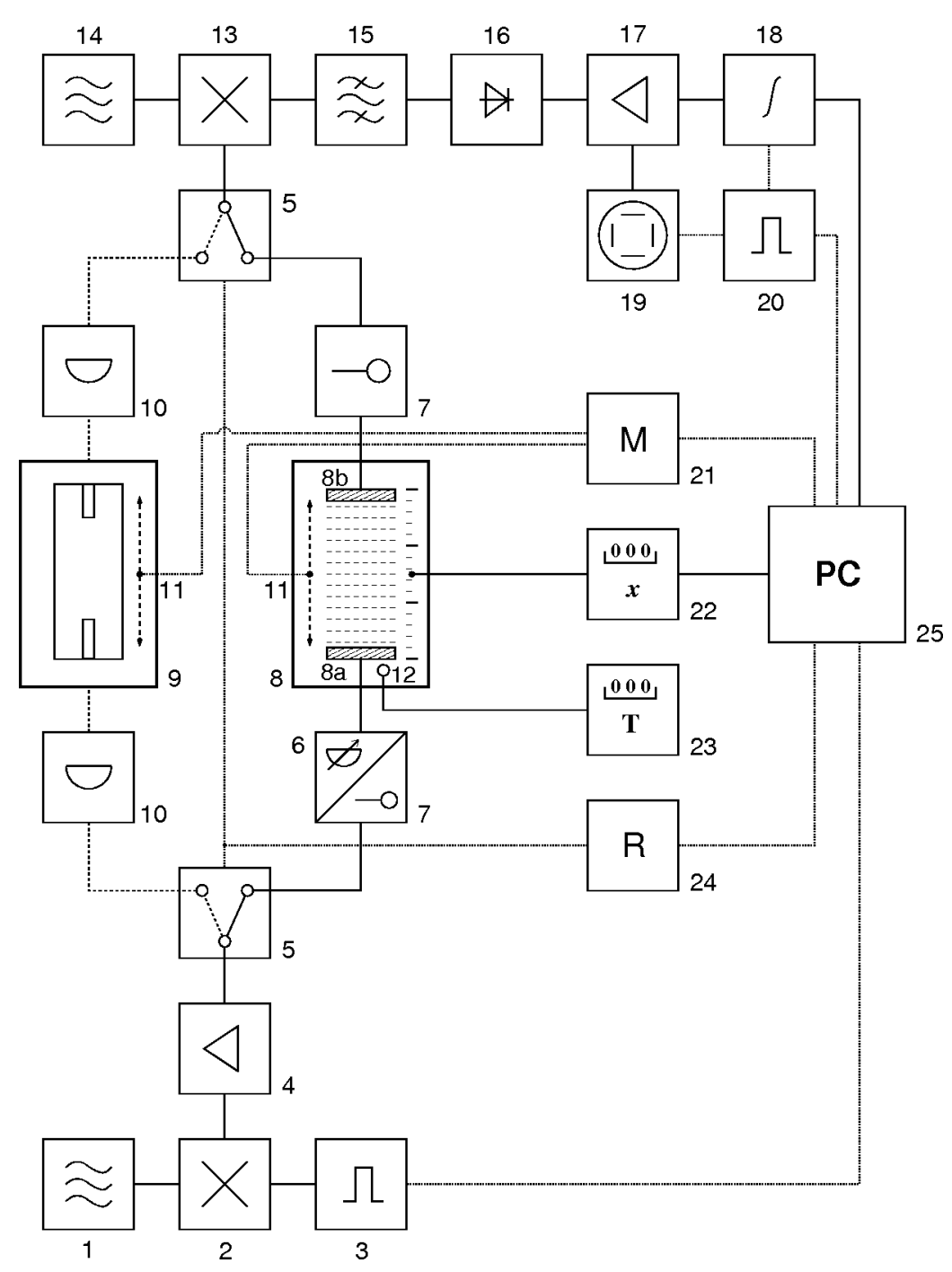

Abbildung 4.12: Blockschaltbild des Messplatzes für das Schwingungspulstransmissionsverfahren. (durchgezogene Linie: Signalweg durch den Messzweig, gestrichtelte Linie: Signalweg durch den Referenzzweig, gepunktete Linien: Steuerleitungen): (1) Synthetisiersender, (2) Pin-Dioden-Schalter oder Dioden-Ringmischer; (3) Pulsgenerator; (4) Verstärker (40dB); (5) Koaxialrelais; (6) variables Dämpfungsglied zur Amplitudenanpassung; (7) Kurzschlussstichleitung zur Impedanzanpassung (PZT-Zelle, 10-MHz-Pulszelle); (8) Messzelle; (8a) Sendewandler; (8b) Empfangswandler; (9) Cut-Off-Dämpfungsglied; (10) Dämpfungsglieder zum Schutz vor Reflexionen; (11) Schrittmotor oder Piezo-Translator; (12) Pt100-Thermofühler; (13-16) Überlagerungsempfänger; (17) Verstärker; (18) Sample-and-Hold-Schaltung und A/D-Wandler; (19) Oszillograph; (20) Pulsgenerator; (21) Motorsteuerung oder D/A-Wandler mit 1kV-Verstärker für Piezotranslator; (22) optischer oder induktiver Taster zur Abstandsmessung; (23) Digitalthermometer; (24) Relaistreiberkarte; (25) Steuerrechner [39]. 
Tabelle 4.2: Übersicht über die Schwingungspulstransmissionszellen. In Klammern sind bei der 10-MHz-Pulszelle neue Daten nach Austausch der Wandler angegeben.

\begin{tabular}{|c||c|c|c|c|}
\hline & $\begin{array}{c}1-\mathrm{MHz}- \\
\text { Pulszelle } \\
{[63]}\end{array}$ & $\begin{array}{c}10-\mathrm{MHz}- \\
\text { Pulszelle } \\
{[65]}\end{array}$ & $\begin{array}{c}\text { Hyperschall- } \\
\text { zelle } \\
{[82],[64]}\end{array}$ & $\begin{array}{c}\text { PZT- } \\
\text { Zelle } \\
{[71],[64]}\end{array}$ \\
\hline Wandlermaterial & Quarz & LiNbO $_{3}$ & LiNbO & ZnO \\
Verzögerung & nein & ja & nein & ja \\
$r_{\mathrm{w}} /[\mathrm{mm}]$ & 20 & 6 & 1,5 & 3,5 \\
$f_{\mathrm{w}} /[\mathrm{MHz}]$ & 1,05 & $10,8(8,0)$ & breitbandig & $\approx 1300$ \\
$f_{n}$ & $(2 n+1) f_{\mathrm{wq}}$ & $(2 n+1) f_{\mathrm{w}}$ & $0,5-2,0 \mathrm{GHz}$ & $0,6-2 \mathrm{GHz}$ \\
$f_{\max } /[\mathrm{MHz}]$ & 100 & $450(400)$ & 2000 & 4600 \\
$\tau /[\mu \mathrm{s}]$ & $4-8$ & $2-4$ & 1,5 & 1,5 \\
\hline Taster & optisch & optisch & optisch & induktiv \\
& MT60 & MT25 & MT 10 & LVDT \\
Tasterposition & axial & axial & $7 \mathrm{~cm}$ & axial \\
$x_{\min } /[\mathrm{nm}]$ & 125 & 125 & 0,5 & 8 \\
$x_{\max } /[\mathrm{nm}]$ & 55000 & 25000 & 1000 & 40 \\
$V /[\mathrm{ml}]$ & $\approx 130$ & $\approx 10$ & $\approx 3$ & $\approx 0.5$ \\
\hline \multicolumn{2}{|r|}{}
\end{tabular}

Die Wandler werden hier von Hand vorsichtig zusammen gefahren, bis die im vorherigen Abschnitt beschriebene $(\lambda / 2)$-Welligkeit nicht mehr zu erkennen ist. Dieser Effekt wird sichtbar, kurz bevor die Wandler miteinander in Kontakt treten. Bei der PZT-Zelle ist zuvor eine Eichung des induktiven Längentasters durchzuführen.

Die nachfolgenden Schritte differieren je nach verwendetem Aufbau etwas; sie werden daher jeweils gesondert behandelt.

\section{Die 1-MHz- und die 10-MHz-Pulszelle}

Für die 1-MHz- und die 10-MHz-Pulszelle wird der gleiche Messplatz verwendet. Die Messung erfolgt an den $(2 n+1)$-ten Oberwellen der Quarz- bzw. $\mathrm{LiNbO}_{3}$-Dickenresonanzen. Der Frequenzbereich wird zu niedrigen Frequenzen durch den Fahrweg, zu hohen Frequenzen durch die Absorption in der Flüssigkeit und die verringerte Energieabstrahlung in den hohen Resonanzmoden eingeschränkt. In Abb. 4.13 ist die 1-MHz-Pulszelle im Querschnitt dargestellt.

Zu Beginn der Messung ist bei geeigneter Frequenz eine Paralleljustierung der Wandlerplatten $(8 \mathrm{a}, \mathrm{b})$ durchzuführen. Dabei wird der untere feststehende Wandler durch die Mikrometerschrauben (14) gegenüber dem oberen Wandler so verkippt, dass die Amplitude des Signals auf dem Oszillographen (Abb.4.12 (19)) maximal wird. Die Wandler sind für jede Messfrequenz wieder neu zu justieren, da die Abstrahlcharakteristika mit der Frequenz variieren.

Nach Erreichen der Zieltemperatur in der Probe ist die Schallgeschwindigkeit zu bestimmen, um für die Sample-and-Hold-Schaltung (Abb.4.12 (18)) die Laufzeit des Signals bei verschiedenen Weglängen berechnen zu können. Dazu wird die Schallgeschwindigkeit 
vorgegeben, so dass die Sample-and-Hold-Schaltung für verschiedene EmpfangswandlerPositionen das empfangene Signal synchronisieren kann.

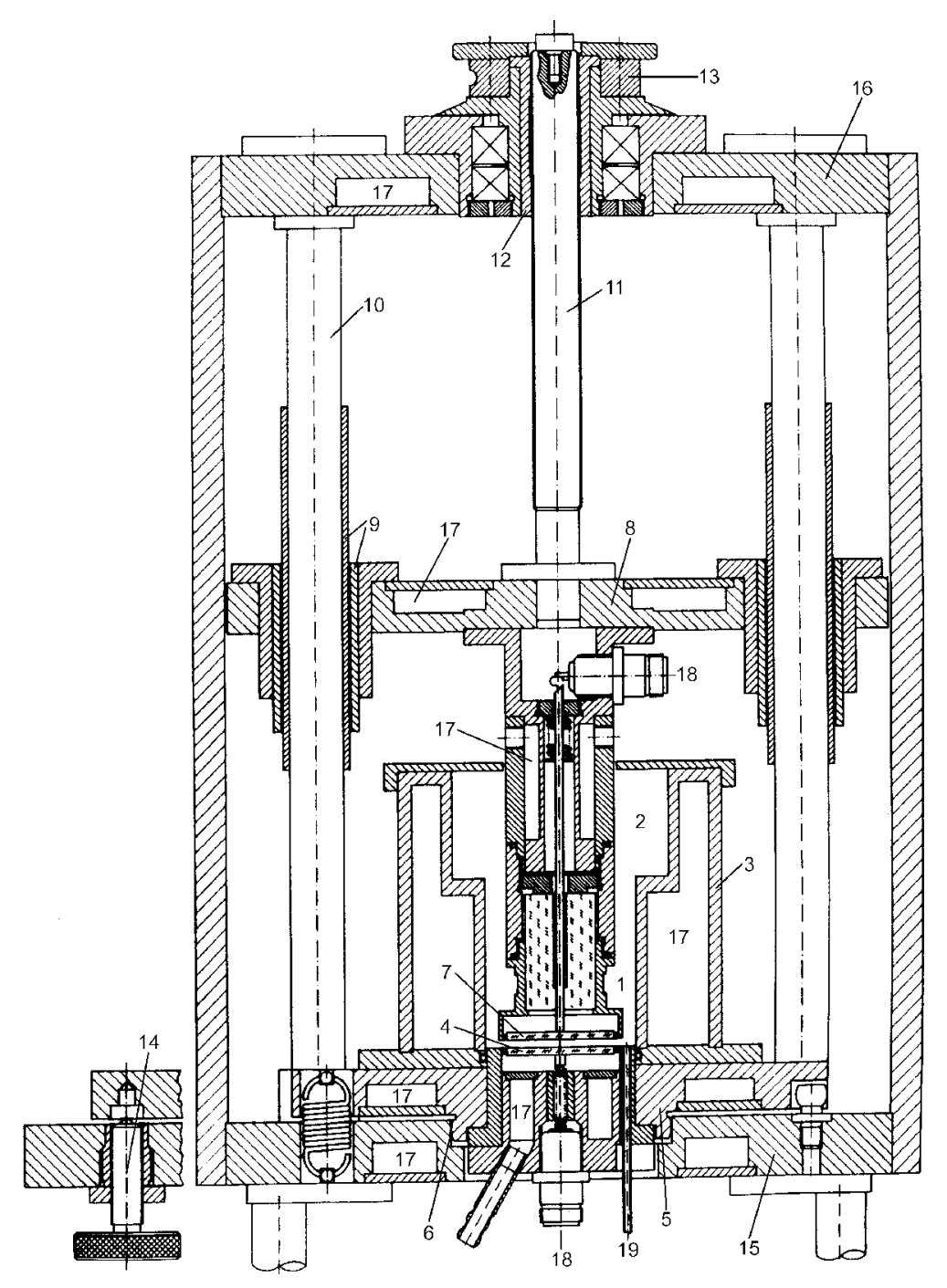

Abbildung 4.13: Querschnitt der 1-MHz-Pulszelle. (1) Messvolumen mit Vorratsbereich; $(2,3)$ Thermostatiermantel; (4) Sendewandler; (5) kippbare Montageplatte; (6) Kugelgelenk; (7) Empfangswandler; (8) verschiebbare Montageplatte; (9) Kugelführung; (10) Führungssäule; (11) Spindel; (12) Mutter; (13) Riemenscheibe zum Antrieb von (11); (14) Schrauben zur Paralleljustierung; (15) Grundplatte; (16) Deckenplatte; (17) Thermostatierkanäle; (18) N-NormAnschlüsse; (19) Ablaufkanüle.

Startabstände und Verstärkung im Überlagerungsempfänger werden so eingestellt, dass für die Maximalamplitude das Signal im Mess- und Referenzzweig einen Wert von $A_{\max }=0,8 \mathrm{~V}$ ergibt. Für höhere Amplituden ist die Übertragungsfunktion des Systems nicht mehr linear. Bei tiefen Frequenzen muss das Signal im Messzweig gegebenenfalls elektronisch gedämpft werden, um einen Abgleich zu erhalten. Desweiteren ist der Überlagerungsempfänger nur in der Lage, Frequenzen zwischen 20 und $1000 \mathrm{MHz}$ zu verarbeiten. Bei tieferen Frequenzen wird ein Signal von $100 \mathrm{MHz}$ nach Durchlaufen der Messzelle hinzu gemischt, um wieder in den Empfangsbereich zu gelangen.

Bei hohen Frequenzen der 10-MHz-Pulszelle werden Kurzschlussstichleitungen (Abb. 4.12 
(7)) im Messzweig eingesetzt, um die Übergangsimpedanz des Wandlers anzupassen; dadurch werden Reflexionen am Impedanzübergang minimiert.

Im Laufe der Arbeit wurden die Wandler der 10-MHz-Pulszelle gegen neue Wandler mit einer Dickenresonanz von $8 \mathrm{MHz}$ ausgetauscht. Dadurch haben sich die Messfrequenzen entsprechend verschoben. Der Austausch hat jedoch keinerlei Auswirkungen auf den Messverlauf oder die Qualität der Daten.

\section{Die tieffrequente Hyperschallzelle}

Das Kernstück der tieffrequenten Hyperschallzelle, dargestellt in Abb. 4.14 schließt nach oben an den Messbereich der 10-MHz-Pulszelle an. Im Gegensatz zu den anderen Apparaturen des Schwingungspulstransmissionsverfahrens, werden hier die Lithiumniobat $\left(\mathrm{LiNbO}_{3}\right)$-Wandler nicht zu Dickenschwingungen angeregt, sondern nach Bömmel und Dransfeld zu Oberflächenschwingungen [19], die zur Schallerzeugung im kontinuierlichen Spektrum zwischen $500 \mathrm{MHz}$ und $2 \mathrm{GHz}$ genutzt werden können. Dabei wird auf dem $\mathrm{LiNbO}_{3}$-Kristall ein inhomogenes elektrisches Feld erzeugt, das das Wandlermaterial zu Schwingungen anregt. Die maximale Feldstärke wird durch abstimmbare Koaxialresonatoren (Abb. 4.14 (5-12)) erreicht, in die die Wandler eingebaut sind.

Der Messablauf entspricht dem zuvor beschriebenen. Aufgrund der kurzen Weglängen ist allerdings keine frequenzabhängige Paralleljustierung nötig, so dass dieser Schritt entfallen kann, nachdem die Wandler einmalig justiert sind. Die Bestimmung der Schallgeschwindigkeit erfolgt nach Gl. (4.27) über die $(\lambda / 2)$-Welligkeit.

Der Überlagerungsempfänger des Hyperschall-Messplatzes wird mit einem externen Hilfsoszillator betrieben.

\section{Die Piezo-Translator-Hyperschallzelle}

Die Piezo-Translator-Hyperschallzelle (PZT-Zelle) wird am gleichen Messplatz betrieben wie die tieffrequente Hyperschallzelle. Wie aus Tab. $4.2 \mathrm{zu}$ entnehmen ist, befinden sich die Wegstrecken in der Probe im Nanometer-Bereich. Die Bewegung der Wandler erfolgt daher nicht mehr mit einem Schrittmotorantrieb, dessen Genauigkeit nicht mehr ausreicht, sondern über einen Piezotranslator. Dabei wird, wie beim piezoelektrischen Ultraschallwandler, die Dickenänderung bei Anlegen eines elektrischen Feldes nach Gl. (4.1) ausgenutzt.

Zur Abstandsbestimmung wird ein induktiver Längentaster benutzt, der vor der Messung über einen „herkömmlichen“ Längentaster kalibriert wird. Dazu wird kontinuierlich in dem Piezotranslator die Spannung zwischen 0 V und 1000 V erhöht.

Nach Kalibrierung des Längentasters kann die eigentliche Messung erfolgen. Der Ablauf entspricht dabei der Messvorschrift für die tieffrequente Hyperschallzelle. 


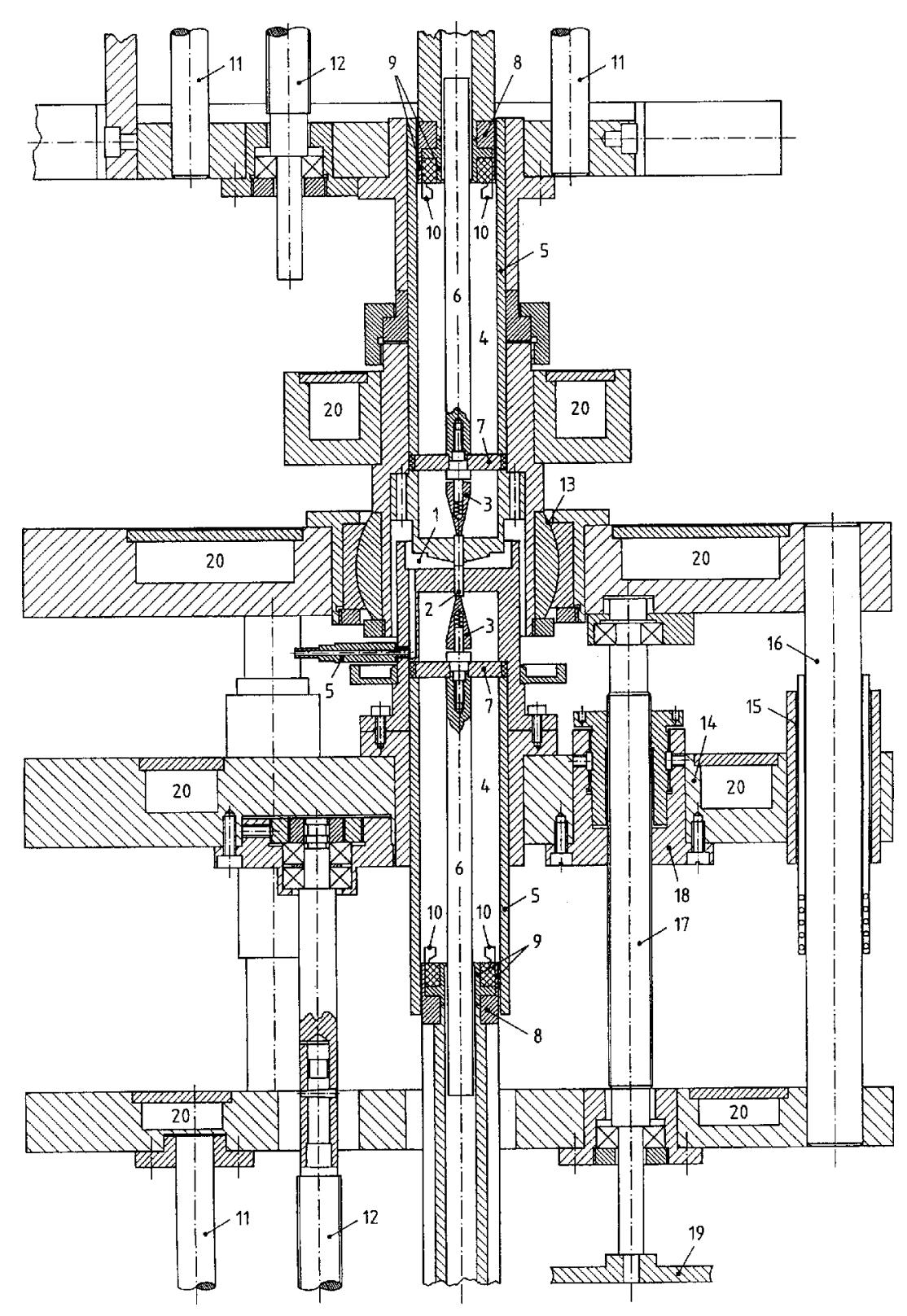

Abbildung 4.14: Querschnitt des zentralen Bereichs der tieffrequenten Hyperschallzelle. (1) Messvolumen; (2) $\mathrm{LiNbO}_{3}$-Stäbe als Schallwandler; (3) federndes Innenleiterende zum Andruck an die Schallwandler; (4) Koaxialresonatoren bestehend aus (5) Resonatoraußenleiter und (6) Resonatorinnenleiter; (7) stützende Plastikscheibe; (8) Kurzschluss; (9) elastische leitende Lamellen; (10) Induktionsschleife zur Ein- bzw. Auskopplung des HF-Signals in den Koaxialresonator; (11) Kugelführungen und (12) Spindel zur Verstimmung der Koaxialresonatoren; (13) Kugelgelenk zur Paralleljustierung der Wandler; (14) Schlitten zur Variation der Zelllänge; (15) Kugelführung und (16) Führungsstange; (17) Spindel zur Abstandsänderung der Wandler; (18) Mutter; (19) Antrieb der Spindel zur Verstimmung der Koaxialresonatoren; (20) Thermostatierkanäle [71]. 
Tabelle 4.3: Übersicht über die Fehler des Pulstransmissionsverfahrens. Die Fehler ergeben sich aus der Streuung der Messdaten und aus Abschätzungen nach [96] und [98].

\begin{tabular}{|c||c|c|}
\hline & Messbereich & Relativer Fehler $\Delta \alpha / \alpha$ \\
\hline \hline 1-MHz-Pulszelle & $\alpha<10 \mathrm{~m}^{-1}$ & $5 \%$ \\
& $\alpha>10 \mathrm{~m}^{-1}$ & $2,5 \%$ \\
\hline 10-MHz-Pulszelle & $f<50 \mathrm{MHz}$ & $1 \%$ \\
& $50 f<300 \mathrm{MHz}$ & $0,7 \%$ \\
& $f>300 \mathrm{MHz}$ & $1,0 \%$ \\
\hline Tieffrequente & $x>20 \mu \mathrm{m}$ & $2 \%$ \\
Hyperschallzelle & sonst & $3 \%$ \\
& $f \leq 620 \mathrm{MHz}$ & $3 \%$ \\
\hline PZT-Zelle & $x>20 \mu \mathrm{m}$ & $<1 \%$ \\
& $2<x<20 \mu \mathrm{m}$ & $1,5 \%$ \\
\hline
\end{tabular}

\subsubsection{Fehlerabschätzung für das Schwingungspulstransmissionsverfahren}

Eine ausführliche Diskussion der Fehler ist bereits in [82] und [96] zu finden. Abweichend von dieser Fehlerbetrachtung sind folgende Probleme aufgetreten.

1-MHz-Pulszelle. Die 1-MHz-Pulszelle benötigt ein großes Probenvolumen aufgrund des Wandlerdurchmessers und der Wandlerabstände.

Für eine Messung von DMPC/Alamethicin stehen ungefähr $60 \mathrm{ml}$ der Probe zur Verfügung. Bei einem Radius des Messvolumens von $r \approx 25 \mathrm{~mm}$ beträgt die Flüssigkeitssäule im Messvolumen ungefähr

$$
x_{\max } \approx 30 \mathrm{~mm}
$$

Bei einer mittleren Dämpfung von $\left(\alpha / f^{2}\right)=40 \cdot 10^{-15}$ und einer Frequenz von $f=10 \mathrm{MHz}$ wird nach Gl. (4.28) ein 3dB-Abfall nach

$$
x_{3 \mathrm{~dB}}=\frac{\ln 2}{\left(40 \cdot 10^{-15}\right) \cdot\left(20 \cdot 10^{-6}\right)^{2}}=43 \mathrm{~mm}
$$

erreicht. Damit muss zum einen der Startabstand der Messung minimiert werden, wodurch der Einfluss der $(\lambda / 2)$-Welligkeit nach Gl. (4.27) sehr groß ist und zum anderen kann für Frequenzen $f \leq 30 \mathrm{MHz}$ über die Messstrecke kein 3dB-Abfall erreicht werden, so dass der Fehler der Messung sehr groß wird. Die Standardabweichungen der gemittelten Messdaten weisen Fehler bis zu 5\% auf. Die Abschätzungen sind in Tab. 4.3 aufgelistet.

Tieffrequente Hyperschallzelle und PZT-Zelle. Die Absorptionskoeffizienten für Frequenzen $f \leq 620 \mathrm{MHz}$ und für $f=1200 \mathrm{MHz}$ liegen in allen Messungen um ca. $5 \%$ über den erwarteten Werten. Die Wandler weisen teilweise Brüche an der Wandleroberfläche auf, so dass unter Umständen keine einheitliche Oberflächenanregung erfolgen kann. Bei 
$f=1200 \mathrm{MHz}$ ist das Signal nur sehr schwach, so dass der Fehler durch ein geringes Signal-Rausch-Verhältnis ansteigt. Die PZT-Zelle ist wegen technischer Probleme nur in sehr wenigen Messungen genutzt worden, daher kann keine Fehlerdiskussion an dieser Stelle erfolgen. Die Daten in dem Frequenzbereich von $1-2 \mathrm{GHz}$ wurden durch Messungen mit der tieffrequenten Hyperschallzelle aufgenommen.

\subsection{Die Schallgeschwindigkeitsmessung}

Am Lipid-Phasenübergang von der Gelphase in die fluide Phase ist nicht nur mit einem Anstieg der Absorption in der Nähe des Phasenübergangs zu rechnen, sondern auch mit einer starken Änderung der Schallgeschwindigkeit aufgrund der Änderung der Kompressibilität der Membran [98]. Die Grundlagen der Schallgeschwindigkeitsmessung (Velocimetrie) sowie ein verbreitetes Verfahren sollen hier vorgestellt werden.

\subsubsection{Grundlagen der Schallgeschwindigkeitsmessung}

Die Schallgeschwindigkeitsmessung liefert aufgrund des Zusammenhangs zwischen der Schallgeschwindigkeit $c_{\mathrm{S}}$ und der adiabatischen Kompressibilität $\kappa_{\mathrm{S}}$ nach Gl. (3.6) Aussagen über die Änderungen der Kompressibilität einer Lösung. Messungen an Natriumchlorid [85] oder komplexen Biomolekülen [22, 23, 24, 25] in Wasser zeigen, dass bereits kleine Substanzmengen zu einer deutlichen Änderung der Kompressibilität der Lösung führen. Der Schlüssel zu diesem Phänomen liegt in der Differenzierung zwischen freiem (bulk) Wasser und Hydratwasser.

Im freien (bulk) Wasser bildet sich ein loses Wasserstoffbrückennetzwerk, dessen Bindungen regelmäßig aufgebrochen und neu formiert werden. Die polaren Wassermoleküle besitzen im Mittel keine Vorzugsrichtung, so dass die Lösung nach außen elektrisch neutral erscheint.

In der Nähe eines geladenen Ions, wie z. B. $\mathrm{Na}^{+}$oder $\mathrm{Cl}^{-}$, ändert sich dieses Verhalten. Aufgrund der Ladungsanziehung zwischen $\mathrm{Na}^{+}$und dem Sauerstoff des Wassers und zwischen $\mathrm{Cl}^{-}$und den Wasserstoffatomen wird eine Vorzugsrichtung vorgegeben.

Durch die stärkere Anziehung zwischen den Bindungspartnern wird der atomare Abstand verkürzt und die Mobilität der Moleküle reduziert. Dieses führt letztendlich zu einer Reduktion der Kompressibilität des Hydratwassers von

$$
\kappa_{\mathrm{S}}^{\text {hyd }} \approx 0,8 \kappa_{\mathrm{S}}^{\text {bulk }} \text { für } \mathrm{NaCl} .
$$

Neben diesen Hydratationseffekten führt auch die Änderung der intrinsischen Eigenschaften des Moleküls zu einer Änderung der Kompressibilität. Es ist leicht einzusehen, dass die Lipide eines Vesikels in der Gelphase eine geringere Kompressibilität besitzen als in der fluiden Phase. Dieser Effekt wird durch Messergebnisse an späterer Stelle quantisiert.

Die Änderung der Schallgeschwindigkeit als Maß für die Änderung der Kompressibilität ist daher eine wichtige Messgröße. Nach [97] lässt sich diese zudem leichter und präziser 
ermitteln als die Messung der absoluten Schallgeschwindigkeit. Es werden durch Differenziation die relativen Größenänderungen eingeführt [97], die auf die Substanzkonzentration $k$ normiert werden:

$$
\begin{aligned}
{\left[c_{\mathrm{S}}\right] } & =\frac{c_{\mathrm{s}}-c_{\mathrm{s}, 0}}{c_{\mathrm{s}, 0} \cdot k} \\
{[\rho] } & =\frac{\rho-\rho_{0}}{\rho_{0} \cdot k} \\
{\left[\kappa_{\mathrm{S}}\right] } & =\frac{\kappa_{\mathrm{S}}-\kappa_{\mathrm{S}, 0}}{\kappa_{\mathrm{S}, 0} \cdot k}
\end{aligned}
$$

mit

$k:$ Konzentration der gelösten Substanz in $\mathrm{mol} / \mathrm{cm}^{3}$

$\left[c_{\mathrm{s}}\right]$ : relative Änderung der Schallgeschwindigkeit,

$c_{\mathrm{s}}, c_{\mathrm{s}, 0}:$ Schallgeschwindigkeit der Lösung und des reinen Lösungsmittels

$[\rho], \rho, \rho_{0}$ : wie zuvor, aber auf die Dichte bezogen

$\left[\kappa_{\mathrm{S}}\right], \kappa_{\mathrm{S}}, \kappa_{\mathrm{S}, 0}$ : wie zuvor, aber auf die adiabatische Kompressibilität bezogen.

Durch Differenziation von Gl. (3.6) lässt sich die Änderung der Kompressibilität darstellen als

$$
\left[\kappa_{\mathrm{S}}\right]=-2\left[c_{\mathrm{S}}\right]-[\rho] .
$$

Die differenziellen Größen $\left[c_{\mathrm{s}}\right]$ und $[\rho]$ lassen sich empirisch bestimmen.

Für die Ermittlung der Schallgeschwindigkeit wird das zuvor beschriebene Resonatorverfahren angewandt. Ausgehend vom idealen Resonator gilt nach Gl. (4.7)

$$
f_{n}=n \frac{c_{\mathrm{s}}}{2 L} \Rightarrow c_{\mathrm{s}}=\frac{2 L}{n} f_{n}
$$

Damit wird Gl. (4.30) zu

$$
\left[c_{\mathrm{s}}\right]=\frac{c_{\mathrm{s}}-c_{\mathrm{s}, 0}}{c_{\mathrm{s}, 0} \cdot k} \approx \frac{f_{n}-f_{n, 0}}{f_{n, 0} \cdot k}(1+\gamma)
$$

mit

$\left[c_{\mathrm{s}}\right]:$ Schallgeschwindigkeitszahl

$c_{\mathrm{s}, 0}, c_{\mathrm{s}}$ : Schallgeschwindigkeit von Referenz und Substanz

$f_{n, 0}, f_{n}$ : Mittenfrequenzen der $n$-ten Resonanz von Referenz und Substanz

$k:$ Konzentration der gelösten Substanz in $\mathrm{mol} / \mathrm{cm}^{3}$

$\gamma$ : Korrekturfaktor gemäß Gl. (4.16) für nicht äquidistante Resonanzen.

Die Bestimmung der relativen Änderung der Schallgeschwindigkeit, die auch als Schallgeschwindigkeitszahl bezeichnet wird, lässt sich auf eine „einfache“ Frequenzmessung zurückführen. Abb. 4.15 zeigt den typischen Aufbau zur Messung der Schallgeschwindigkeit. 


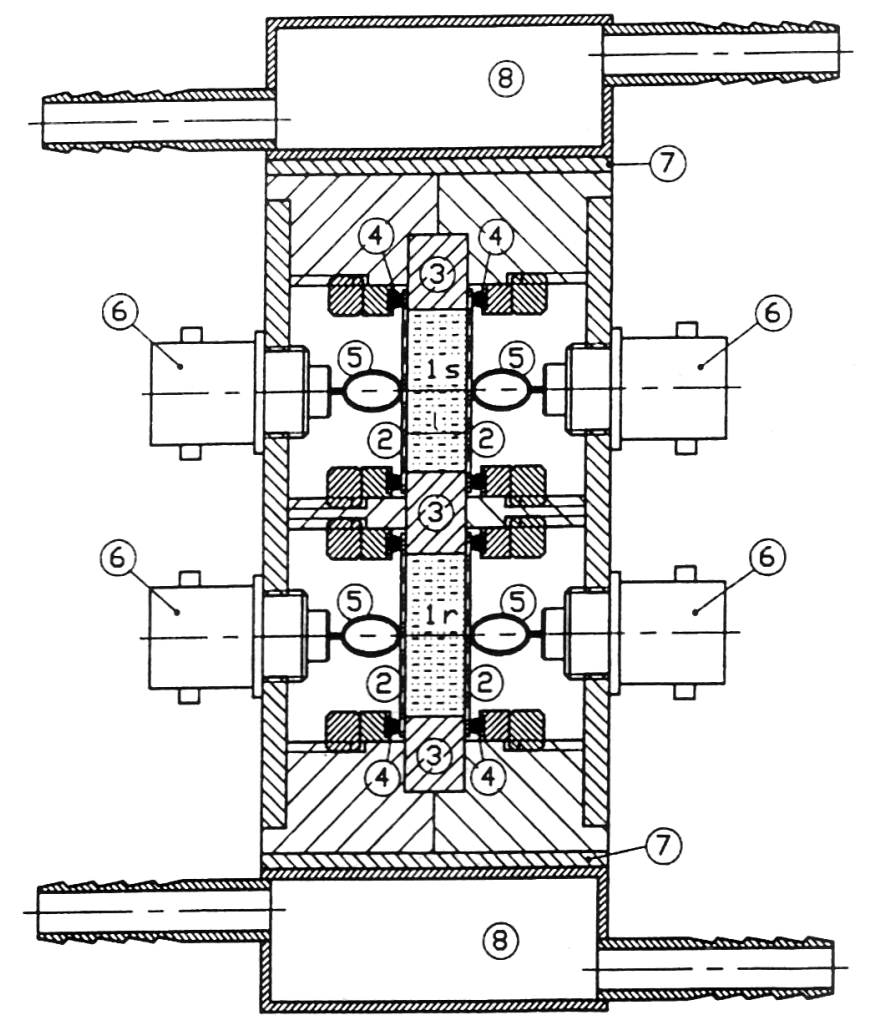

Abbildung 4.15: Querschnitt durch die Doppel-Resonatorzelle. (1s), (1r) Messvolumina für Substanz- und Referenzmessung; (2) Quarzwandler; (3) Abstandsplatten aus V2A-Stahl; (4) O-Ringe; (5) rückseitige Wandlerkontaktierungen über Pt-Federn; (6) BNC-Anschluss; (7) Peltier-Element; (8) Thermostatierkanäle.

Die Doppelresonatorzelle besteht aus zwei baugleichen Resonatormesszellen, die miteinander im thermischen Gleichgewicht stehen. Die Temperaturregulierung wird über ein Wasserbad und ein Peltier-Element gewährleistet. Das Peltier-Element ist dabei mit einem elektronischen Thermometer verbunden, so dass über eine Regelautomatik kleine Temperaturschwankungen ausgeglichen werden können.

Die Referenzzelle (1r) wird mit einer Substanz mit bekannten physikalischen Eigenschaften befüllt. Im Falle der Lipid/Wasser-Lösungen wird Wasser als Referenz verwendet. Für die Messung wird die $n$-te Resonanz bei der Frequenz $f_{n, 0}$ ausgewählt. Für Frequenzen in der Nähe der halben Quarzfrequenz $f_{n} \approx f_{\mathrm{q}} / 2$ wird der Korrekturterm $\gamma$, der hauptsächlich Gl. (4.16) berücksichtigt, vernachlässigbar.

Für die Messung in der Zelle (1s) wird ebenfalls die $n$-te Resonanz bei $f_{n}$ ausgewählt. Über eine Lorentzanpassung der Resonanz wird die Mittenfrequenz und die Halbwertsbreite kontinuierlich in Referenz- und Substanzzelle bestimmt. Die am III. Physikalischen Institut entwickelte Schallgeschwindigkeitsmessapparatur [75] kann die Schallgeschwindigkeiten für jeweils eine Resonanz in Abhängigkeit von der Temperatur aufnehmen. 


\subsubsection{Das kommerzielle Spektrometer HR-US 102}

Das kommerzielle Spektrometer Ultrasonic Scientific HR-US 102 [22, 23, 24, 25, 26] erlaubt, simultan die Mittenfrequenzen und Halbwertsbreiten von acht verschiedenen Resonanzen aufzuzeichnen. Damit lassen sich sowohl Dispersions- als auch Absorptionskurven der Substanz in einem engen Frequenzbereich darstellen. Neben der temperaturabhängigen Messung der Schallgeschwindigkeit kann mit dem HR-US 102 auch eine konzentrationsabhängige Messung (Titration) durchgeführt werden.

Mit Hilfe der Titration Kinetics Accessories kann während der Messung die Konzentration einer Substanz im Probevolumen durch schrittweise Zugabe des Titranten erhöht werden. Die Schrittweite hängt von der Größe der Mikroliterspritze (5) in Abb. 4.16 ab.

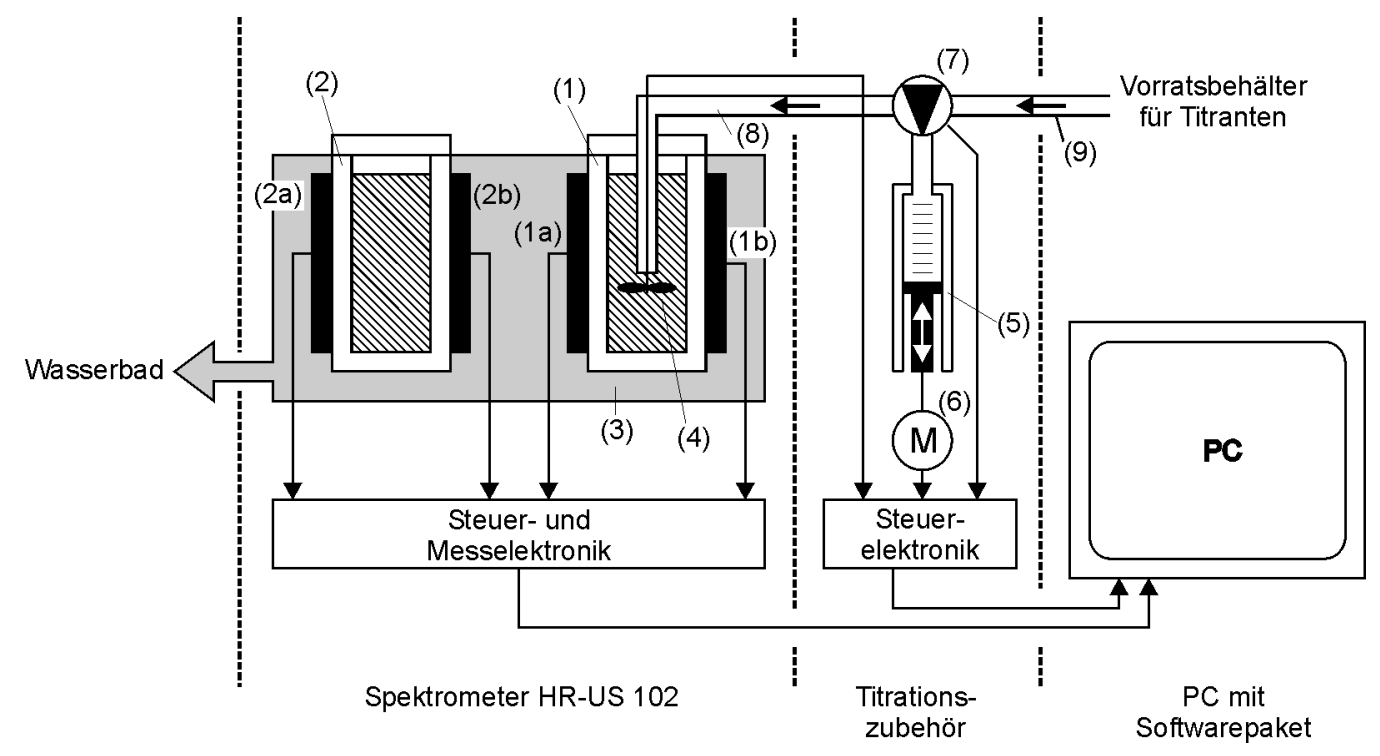

Abbildung 4.16: Skizze des Aufbau für Titrationsexperimente. (1) Zelle 1, Referenzzelle: Messküvette mit Probenvolumen (schraffiert); (1a), (1b) Sende- und Empfangswandler; (2) Zelle 2, wie (1); (3) Thermostatierhülle; (4) Rührvorrichtung; (5) Mikroliterspritze; (6) Motor zur Bewegung des Spritzenkolbens; (7) Ventil zur Umschaltung zwischen Lade- und Titrationsschlauch;

(8) Titrationsschlauch zur Messküvette; (9) Ladeschlauch vom Vorratsbehälter des Titranten.

Die Messküvetten $(1,2)$ werden über ein externes Wasserbad thermostatiert. Zur Kontrolle der Temperaturstabilität wird Zelle 2 mit bidestilliertem Wasser befüllt und die zeitliche Änderung der Mittenfrequenz protokolliert.

Die Titration wird in Zelle 1 durchführt. Dazu wird diese mit $1 \mathrm{ml}$ einer extrudierten reinen DMPC-Lösung befüllt und auf die Messtemperatur thermostatiert. Die exakte Konzentration variiert leicht zwischen den Proben; diese Abweichungen werden aber in den Rechnungen berücksichtigt. Im HR-US-Messprogramm werden mehrere Resonanzen ausgewählt, deren Mittenfrequenzen $f_{n}$ und Halbwertsbreiten $\Delta f_{n}$ im Laufe der Messung aufgezeichnet werden.

Aufgrund seines hydrophoben Verhaltens wird Alamethicin für die Titration in Ethanol gelöst. Um den Effekt auf die Schallgeschwindigkeit durch die Zugabe von Alamethicin zu der DMPC-Suspension bestimmen zu können, muss vorher eine Referenztitration von reinem Ethanol zu der Lipidsuspension durchgeführt werden. Der Einfluss des Alamethicins 
kann so durch Differenzenbildung der Mittenfrequenzen von Referenz- und Substanzmessung bestimmt werden.

Der Fehler in der Schallgeschwindigkeit wird nach Buckin et al. [25] mit $\Delta c_{\mathrm{s}} / c_{\mathrm{s}}=3 \cdot 10^{-7}$ für Kolloide und Biopolymere angegeben. Bei einer Schallgeschwindigkeit von $c_{\mathrm{s}}=1500 \mathrm{~m} / \mathrm{s}$ beträgt der Fehler $\Delta c_{\mathrm{s}} \approx 0,5 \mathrm{~mm}$. Der Fehler in der Konzentration der Alamethicinlösung, der durch Messfehler beim Pipettieren entsteht, kann mit ungefähr 0,5\% abgeschätzt werden. Die Genauigkeit der Titrationsschritte ist nicht bekannt, sollte aber deutlich unter 1\% liegen. Ebenso kann der Konzentrationsfehler beim Ansetzen der DMPC-Lösung mit $1 \%$ abgeschätzt werden.

\subsubsection{Aufbau des Velocimetrie-Messplatzes}

Da sowohl die Apparatur zur Schallgeschwindigkeitsmessung nach [75], als auch das Ultrasonic Scientific HR-US 102 für die Messungen nur begrenzt zur Verfügung standen, wurde auf Basis des Programms NWAControl ein vereinfachter Velocimetrie-Messplatz aufgebaut, der in Abb. 4.17 dargestellt ist.

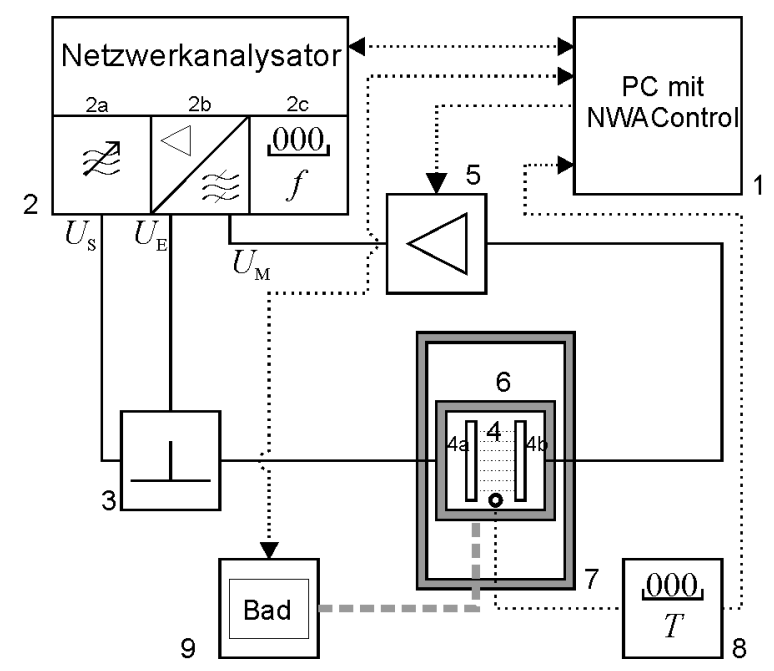

Abbildung 4.17: Blockschaltbild des Velocimetrie-Messplatzes. Die durchgehende Linie symbolisiert den Weg des Messsignals, gepunktete Linien repräsentieren Mess- und Steuerleitungen, die gestrichelten Linien optionale Komponenten. (1) Steuerrechner; (2) Netzwerkanalysator mit (2a) Generator, (2b) Zweikanal-Überlagerungsempfänger und (2c) Frequenzzähler; (3) Signalteiler; (4) 5-MHz-Resonator mit (4a) Sende- und (4b) Empfangswandler (5) regelbarer Verstärker mit paralleler Schnittstelle; (6) innere thermische Isolierung; (7) äußere Thermostatierung mit Wasserbad; (8) Digitalthermometer mit externem Pt100-Messfühler und RS232-Schnittstelle; (9) ansteuerbarer Thermostat mit RS232-Schnittstelle zur Thermostatierung des Resonators.

Bei dem hier vorgestellten Verfahren wird die Idee der Simultan-Messung in einer Doppelzelle zugunsten eines einfacheren Aufbaus wieder verworfen. Stattdessen wird wie beim „normalen“ Resonatorverfahren wieder eine getrennte Messung von Referenz und Substanz nacheinander in einer Messzelle durchgeführt.

Die Vorteile dieser Methode liegen auf der Hand: zum einen wird in derselben Zelle gemessen, weshalb unterschiedliche Zellgeometrien oder Wandlereigenschaften ausgeschlossen 
werden können, zum anderen ist dieses Verfahren mit nahezu jedem Resonator anwendbar. Für die durchgeführten Velocimetrie-Versuche wird ein planar-konkaver 5-MHz-Resonator verwendet, der sich schon zuvor bei manuellen Schallgeschwindigkeitsmessungen [41] bewährt hat.

Wie in Abb. 4.17 dargestellt ist, wird der Resonator (4) durch ein regelbares Wasserbad (9) thermostatiert. Der Resonator wird in eine Styropor-Ummantelung (6) gesetzt, um das zu thermostatierende Volumen gering zu halten. Die äußere Thermostatierhülle (7) wird mit einem zweiten Thermostaten (nicht dargestellt) auf einer konstanten Temperatur gehalten, um den Einfluss von Temperaturschwankungen zu minimieren.

\subsubsection{Auswertung der Schallgeschwindigkeitsdaten}

Über ein Skript, das von NWAControl gelesen wird, wird die zu messende Temperaturkurve vorgegeben. Dabei können für verschiedene Temperaturintervalle (Ramps) spezifische Einstellungen angegeben werden, wie aus Abb. C.1 im Anhang hervorgeht. Zuvor wird manuell ein Scan-Bereich um die $n$-te Resonanz ausgewählt. Dieser Bereich wird während der temperaturabhängigen Messung kontinuierlich der Änderung der Mittenfrequenz angepasst.

Nach der Messung von Referenz und Substanz werden die Resonanzdaten an das Programm Velocimetry übergeben, das aus den Daten die Schallgeschwindigkeit berechnet.

Aus den Mittenfrequenzen der Referenzmessung wird die Zelllänge $L$ und die Ordnung $n$ der Resonanz bestimmt. Es wird hierbei eine lineare Änderung der Zelllänge mit der Temperatur angenommen:

$$
L(T)=L_{0}\left(1+\alpha_{\mathrm{L}} T\right)
$$

Die Länge $L_{0}$ und der lineare Ausdehnungskoeffizient $\alpha_{\mathrm{L}}$ werden nach Gl. (4.7) und der Korrektur Gl. (4.16) bestimmt. Dieses setzt eine genaue Kenntnis der temperaturabhängigen Schallgeschwindigkeit und Dichte der Referenz voraus. Diese Daten können anhand von [18] und [67] für Wasser berechnet werden. In Abb.4.18 ist eine Auswertung der Zelllängenkalibrierung dargestellt.

Mit Hilfe des Skriptes wird lediglich eine Temperatur für das Wasserbad vorgegeben, d. h. im Allgemeinen liegt durch diverse Wärmeverluste am Resonator eine leicht unterschiedliche Temperatur $a^{2}$. Selbst wenn für Referenz- und Substanzmessung dasselbe Temperaturskript verwendet wird, so ist nicht gewährleistet, dass die korrespondieren Frequenzen aufgrund z. B. unterschiedlicher Umgebungstemperaturen bei gleichen Temperaturen liegen. Das Programm Velocimetry sucht daher anhand der Temperaturen korrespondierende Mittenfrequenzen und berechnet die Frequenz mittels einer linearen Interpolation zwischen den benachbarten Datenpunkten.

\footnotetext{
${ }^{2}$ Es sei denn, die Temperatur des Wasserbades wird über einen externen Thermofühler geregelt. Ein solcher ist als Zubehör für diverse Thermostaten von Lauda erhältlich.
} 


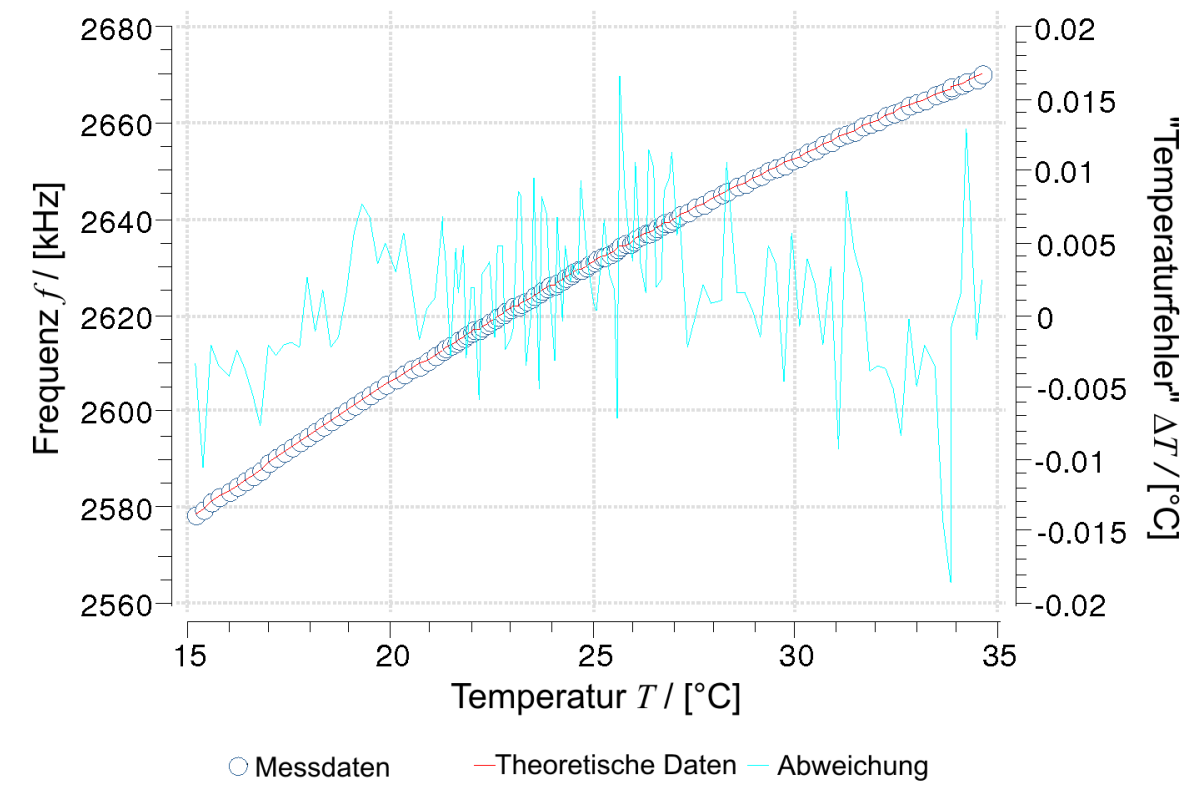

Abbildung 4.18: Fehler der Zelllängenkalibrierung. In dieser Darstellung wurde die Abweichungen der gemessenen Frequenzen $(-)$ von den theoretisch vorhergesagten Frequenz $(0)$ nach $[18,67]$ in Temperaturfehler $(-)$ umgerechnet. Die Fehler liegen bei $\Delta T \leq 0,02 \mathrm{~K}$. Dieses liegt innerhalb des Messfehler des Thermometers. Die Messung erfolgte mit bidestilliertem Wasser zwischen $15^{\circ} \mathrm{C}$ und $35^{\circ} \mathrm{C}$.

\subsubsection{Fehlerabschätzung}

Addiert man die Temperaturfehler nach Abb.4.18 für Referenz- und Substanzmessung, so ergibt sich für $\Delta T \approx 0,04 \mathrm{~K}$ für Wasser bei $25^{\circ} \mathrm{C}$ eine Schallgeschwindigkeit von $c_{\mathrm{s}}=1496,70 \pm 0,107 \mathrm{~m} / \mathrm{s}$. Der Fehler der Messung liegt damit bei $\Delta c_{\mathrm{s}} / c_{\mathrm{s}} \approx 7 \cdot 10^{-5}$. Wie später gezeigt wird, beträgt die relative Schallgeschwindigkeitsänderung für Lipid-/ Wasser-Suspensionen $\left(c_{\mathrm{s}, \mathrm{w}}-c_{\mathrm{s}, \mathrm{DMPC}}\right) / c_{\mathrm{s}, \mathrm{w}} \approx 7 \cdot 10^{-4}$. Der Fehler des Messverfahrens für Lipid/ Wasser-Suspensionen muss daher mit $10 \%$ für diese Änderung angegeben werden. 


\subsection{Densitometrie}

\subsubsection{Geräteeigenschaften und Messprinzip}

Während des Phasenübergangs von der Gelphase in die fluide Phase ist eine messbare Dichteänderung zu erwarten. Für die Densitometrie wurde das Dichtemessgerät Anton Paar DMA 5000 verwendet. Die Vorteile des Gerätes sind [3]

- ein geeigneter Messbereich von $0<\rho<3 \mathrm{~g} / \mathrm{cm}^{3}$ für $0<T<90{ }^{\circ} \mathrm{C}$

- hohe Messgenauigkeit mit einer Standardabweichung $\Delta \rho=10^{-6} \mathrm{~g} / \mathrm{cm}^{3}$,

- hohe Temperaturstabilität $\Delta T=0,001 \mathrm{~K}$,

- geringes Probenvolumen $V \approx 1 \mathrm{ml}$,

- schneller Messvorgang $\tau_{\text {mess }} \approx 40 \mathrm{~s}$.

(a) Seitenansicht

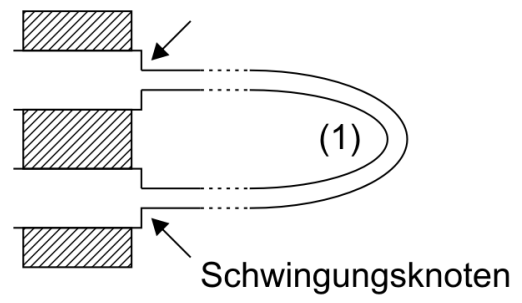

(b) Aufsicht

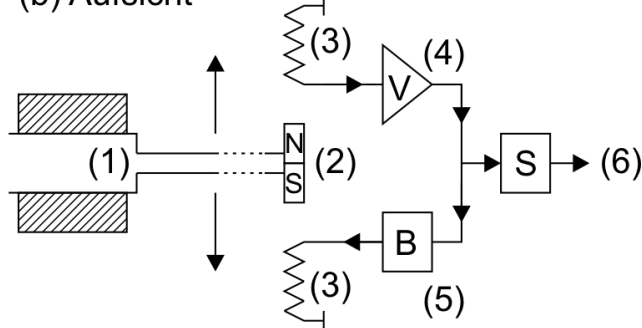

Abbildung 4.19: Funktionsprinzip des Densitometers. (1) Kapillare; (2) Magnet; (3) elektrische Spulen; (4) Verstärker; (5) Begrenzer; (6) Schmitt-Trigger. Die Kapillare wird über den Magnet zwischen den elektrischen Spulen zu Schwingungen angeregt. Verstärker und Begrenzer entdämpfen das Schwingungssystem und begrenzen die Amplitude. Die Schwingung wird über den Schmitt-Trigger in einen Rechteckpuls verwandelt.

Das Densitometer arbeitet nach dem Biegeschwinger-Verfahren [69, 90]. Dabei wird eine U-förmige Kapillare über einen Magneten zu Eigenschwingungen angeregt, wie in Abb. 4.19 dargestellt.

Für eine ungedämpfte, harmonische Schwingung erhält man die Resonanzfrequenz

$$
f=\frac{1}{2 \pi} \sqrt{\frac{k}{m}}=\frac{1}{2 \pi} \sqrt{\frac{k}{m_{0}+\rho V}}
$$

mit

$$
\begin{aligned}
f & : \text { Resonanzfrequenz der Kapillarschwingung } \\
k & : \text { Gerätespezifische Federkonstante } \\
m & : \text { Masse der befüllten Kapillare } \\
m_{0} & : \text { Masse der ungefüllten Kapillare } \\
\rho & : \text { Dichte der Probensubstanz } \\
V & : \text { Volumen der Kapillare. }
\end{aligned}
$$


Die Dichte ergibt sich mit dieser Beziehung zu

$$
\rho=A \tau^{2}-B
$$

mit

$$
A=k /\left(4 \pi^{2} V\right), \quad B=m_{0} / V, \quad \tau=f^{-1} .
$$

Die Parameter $A$ und $B$ sind gerätespezifische Parameter, die über eine Kalibrierungmessung mit einer bekannten Substanz bestimmt werden können. Damit kann die Dichte über die Messgröße $\tau=f^{-1}$ bestimmt werden.

Die Temperatur der Messkapillare wird über ein Peltier-Element stabilisiert. Es stehen drei „Geschwindigkeiten“ für den Temperaturangleich zur Verfügung (langsam, mittel und schnell).

\subsubsection{Ablauf der Messung}

Nach dem Reinigen des Messgerätes mit Methanol oder anderen geeigneten Lösungsmitteln und einem anschließenden Trocknen wird die Kapillare langsam mit der Probenflüssigkeit befüllt. Luftblasen sind hierbei unbedingt zu vermeiden, da sich dadurch die Masse des Probenvolumens sehr stark ändert. Nach dem Befüllen werden über die Menüsteuerung des Anton Paar DMA 5000 die Messparameter gesetzt. Im Rahmen dieser Arbeit wurden Dichtemessungen über einen Temperaturbereich von $10^{\circ} \mathrm{C}$ bis $40^{\circ} \mathrm{C}$ durchgeführt. Es wurden Temperaturschritte von $0,1^{\circ} \mathrm{C}$ oder $0,2^{\circ} \mathrm{C}$ vorgegeben. Da die Wärmekapazität in der Nähe des Phasenübergangs stark ansteigt, ist ein mittlerer oder langsamer Temperaturangleich zu wählen, um ein thermisches Gleichgewicht in der Probe zu gewährleisten.

Während der Messung werden kontinuierlich Temperatur $T$, absolute Dichte $\rho$ und relative Dichte $\rho_{\text {rel }}=\left(\rho / \rho_{\mathrm{H}_{2} \mathrm{O}}\right)$ über die serielle Schnittstelle von einem Computerprogramm ausgelesen.

\subsubsection{Messfehler}

Laut Herstellerangaben [3] beträgt der Messfehler in der Temperatur $\Delta T \approx 0,001 \mathrm{~K}$. Für die Auflösung der Dichtedaten ergibt sich eine Auflösunggrenze von $10^{-6} \mathrm{~g} / \mathrm{cm}^{3}$. Während des Phasenübergangs ist mit einer Änderung der relativen Dichte $\Delta \rho_{\text {rel }} \approx 4 \cdot 10^{-4}$ pro Milligramm DMPC pro Milliliter zu rechnen. Der Fehler des Verfahrens liegt daher für Lipidkonzentration von $c_{\mathrm{DMPC}}=5 \mathrm{mg} / \mathrm{ml}$ bei weniger als $1 \%$. Anhand der Daten ist aber schon deutlich die Auflösungsgrenze des Messgerätes zu erkennen. 


\section{Probenpräparation}

\subsection{Das Ansetzen der Lipidsuspension}

Die im Rahmen dieser Arbeit verwendeten Chemikalien sind in Tab. 5.1 aufgeführt. Es wurde für alle Suspensionen ein Ansatz von

$$
c_{\ell+z}=5 \mathrm{mg} / \mathrm{ml}
$$

benutzt, wobei $\ell$ ein Lipid und $z$ ein Zusatz ist. Das Verhältnis $\ell: z$ wird als Molenbruch gegeben. Die Berechnung der Molmassen ergibt sich wie folgt

$$
\begin{aligned}
& m_{\ell}=c_{\ell+z} V \frac{M_{\ell} x_{\ell}}{M_{\ell} x_{\ell}+M_{z} x_{z}} \\
& m_{z}=c_{\ell+z} V \frac{M_{z} x_{z}}{M_{\ell} x_{\ell}+M_{z} x_{z}}=c_{\ell+z} V\left(1-m_{\ell}\right)
\end{aligned}
$$

mit

$$
\begin{aligned}
m_{\ell}, m_{z} & : \text { einzuwiegende Masse des Lipids und des Zusatzes } \\
c & : \text { Gesamtkonzentration von Lipid und Zusatz } \\
V & : \text { Gesamtvolumen } \\
M_{\ell}, M_{z} & : \text { Molmasse von Lipid und Zusatz } \\
x_{\ell}, x_{z} & : \text { Molenbruch von Lipid und Zusatz. }
\end{aligned}
$$

Zu Beginn der Präparation wird das pulverförmige Lipid mit einer Feinwaage nach Gl. (5.1) auf einem Teflon-Wägeschälchen eingewogen. Die Waage besitzt eine Genauigkeit von 0,1 mg. Das Lipid wird nun in einen flachen Messkolben gegeben. Pulverreste, die im Wägeschälchen haften bleiben, sind mit reinem Ethanol vorsichtig in den Kolben zu geben.

Werden pulverförmige Zusätze zu dem Lipid hinzugegeben, so ist dieser Schritt für den Zusatz zu wiederholen.

Das Alamethicin wird in vor der Verwendung in Ethanol gelöst, um eine bessere Dosierung zu gewährleisten, und mittels einer Messpipette in den Messkolben zu dem Lipid gegeben. Die Messpipetten besitzen eine Genauigkeit von ca. 0,1\% des maximalen Füllvolumens. Im Allgemeinen wurde eine $200 \mu \mathrm{l}$-Pipette mit einer Genauigkeit von $0,1 \mu \mathrm{l}$ verwendet. Bei einer Lösung von typischerweise $25 \mathrm{mg}$ Alamethicin in $1 \mathrm{ml}$ Ethanol ergibt sich hiermit eine rechnerische Genauigkeit entsprechend 2,5 $\mu \mathrm{g}$. 
$\mathrm{Zu}$ dem Gemisch aus Lipid und Zusatz werden $50 \mathrm{ml}$ Chloroform gegeben. Das Lösungsmittel wird $18-24 \mathrm{~h}$ bei Unterdruck $(\leq 10 \mathrm{mmHg})$ und $30^{\circ} \mathrm{C}$ im Vakuumofen verdampft. Das trockene Lipidgemisch sammelt sich an der Innenseite des Messkolbens.

Nun wird die errechnete Menge an destilliertem Wasser abgewogen und hinzugegeben. Die Genauigkeit der verwendeten Waage liegt bei $1 \mathrm{mg}$. Lipid und Wasser werden gut durchmischt. Dabei bilden sich spontan mehrschalige Vesikel (Multilamellar Vesicles, MLV). Die Suspension wird anschließend für ungefähr 60 Minuten in ein Ultraschallbad gestellt. Durch das intensive Schallfeld werden die Vesikel zu Stößen angeregt, so dass diese kontinuierlich aufbrechen und neu verschmelzen. Durch diesen Prozess wird die Bildung unilamellarer Vesikel begünstigt (Large Unilamellar Vesicles, LUV).

Die gebildeten Vesikel zeigen keine einheitliche Größenverteilung. Um dieses zu erreichen ist die Suspension vor dem Gebrauch zu extrudieren. Für die Extrusion wurde ein LiposoFast Basic-Extruder von Avestin [56, 80, 81] verwendet, der im nächsten Abschnitt beschrieben wird.

Nach einer Extrusion, die zur einer homogenen Größenverteilung der Vesikel führt, zeigt sich die Suspension aufgrund des Tyndall-Effektes [31] als klare, bläulich opaleszierende Flüssigkeit. Vor dem Verwenden in den Messapparaturen ist die Suspension zu entgasen. Dazu bieten sich grundsätzlich zwei Möglichkeiten an:

1. Entgasen bei Unterdruck. Die Suspension wird nach der Extrusion für mehrere Minuten in den Vakuumofen gestellt und der Luftdruck auf ein Minimum reduziert. In der Suspension befindliche Blasen expandieren und platzen. Diese Methode ist sehr effektiv, jedoch wird durch die Reduktion des Umgebungsdrucks die Siedetemperatur des Wassers stark reduziert, so dass dieses verdampft. Dadurch verändert sich die Konzentration der Suspension.

2. Entgasen durch Ultraschall. Bei diesem Verfahren werden die Luftblasen durch kontinuierliche Stöße zum Platzen gebracht. Versuche zeigen, dass die Suspension nach ungefähr 60 min hinreichend entgast ist. Aufgrund des Aufbrechens und der Neuformierung der Vesikel kann es zu einer Verbreiterung der Größenverteilung der Vesikel kommen. Die Unilamellarität bleibt aber weitestgehend erhalten, wie man leicht an der Opaleszenz erkennt. Die Extrusion kann im geschlossenen Gefäß erfolgen, so dass keine Konzentrationsänderung der Suspension stattfindet.

Während der Messungen zeigten sich keine offensichtlichen Nachteile durch das Entgasen mittels Ultraschall, so dass dieses Verfahren in den meisten Messreihen Verwendung fand. 
Tabelle 5.1: Übersicht über die verwendeten Chemikalien. Die Molmassen und Reinheit sind den Herstellerangaben entnommen.

\begin{tabular}{|c|c|}
\hline DMPC & 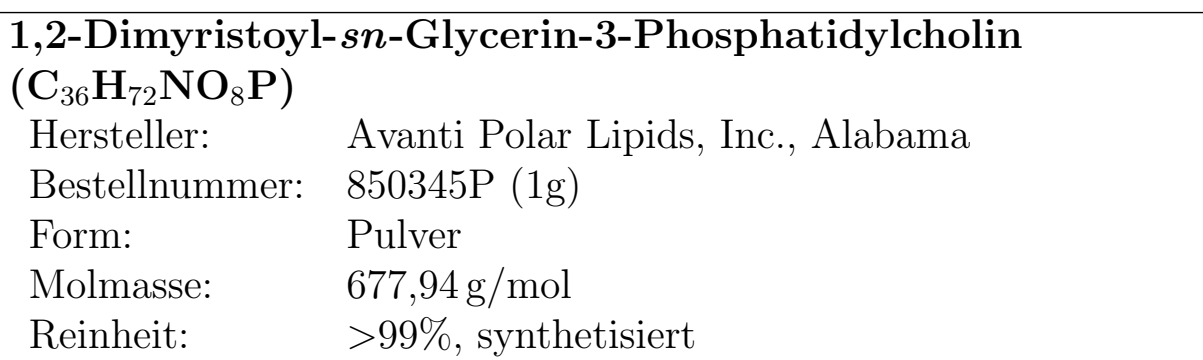 \\
\hline Cholesterol & $\begin{array}{ll}\text { Cholesterol }\left(\mathbf{C}_{27} \mathbf{H}_{46} \mathbf{O}\right) \\
\text { Hersteller: } & \text { Fluka; Sigma-Aldrich, Inc., Missouri } \\
\text { Bestellnummer: } & 26732 \\
\text { Form: } & \text { Pulver } \\
\text { Molmasse: } & 386,65 \mathrm{~g} / \mathrm{mol} \\
\text { Reinheit: } & >99 \%, \text { aus Lanolin }\end{array}$ \\
\hline Alamethicin & $\begin{array}{ll}\text { Alamethicin } & \text { (bestehend aus mehreren Alamethicin- } \\
\text { Homologen) } & \\
\text { Hersteller: } & \text { Sigma; Sigma-Aldrich, Inc, Missouri } \\
\text { Bestellnummer: } & \text { A-4665 } \\
\text { Form: } & \text { Pulver } \\
\text { Molmasse: } & 1960^{\mathrm{a}}, 1959^{\mathrm{b}} \mathrm{g} / \mathrm{mol} \\
\text { Reinheit: } & 85 \%^{\mathrm{a}}, 12 \%^{\mathrm{b}} \text {, aus Trichoderma viride } \\
\end{array}$ \\
\hline Chloroform & $\begin{array}{ll}\text { Chloroform }\left(\mathbf{C H C l}_{3}\right) \\
\text { Hersteller: } & \text { Sigma; Sigma-Aldrich, Inc, Missouri } \\
\text { Bestellnummer: } & \text { C-5312 } \\
\text { Molmasse: } & 119,4 \mathrm{~g} / \mathrm{mol} \\
\text { Reinheit: } & >99,9 \%\end{array}$ \\
\hline Ethanol & $\begin{array}{ll}\text { Ethanol }\left(\mathbf{C}_{2} \mathbf{H}_{5} \mathbf{O H}\right) \\
\text { Hersteller: } & \text { Merck kGaA, Darmstadt } \\
\text { Bestellnummer: } & 100986 \\
\text { Molmasse: } & 46,07 \mathrm{~g} / \mathrm{mol} \\
\text { Reinheit: } & >99,9 \%\end{array}$ \\
\hline
\end{tabular}




\subsection{Präparation der Vesikel}

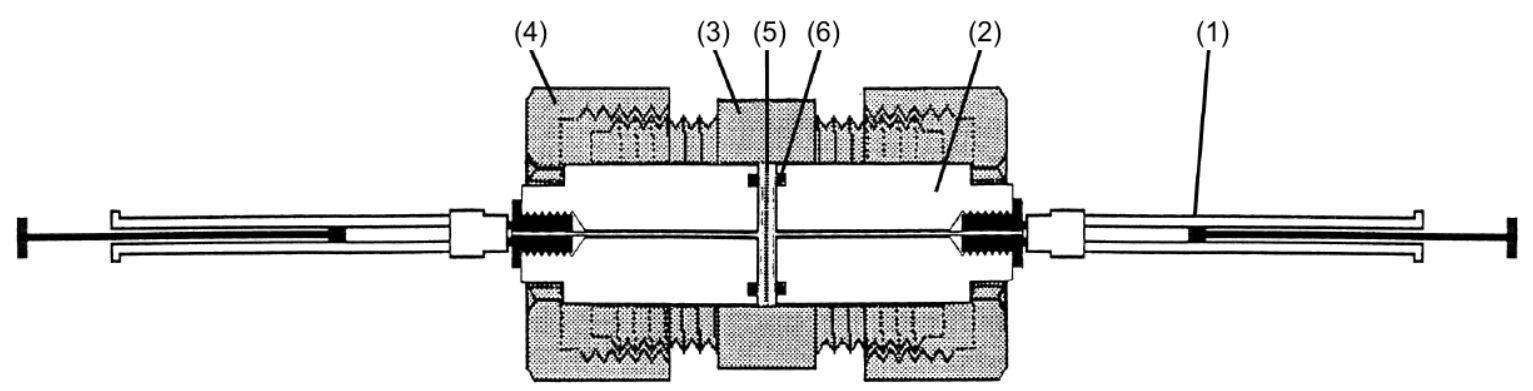

Abbildung 5.1: Querschnitt des LiposoFast Extruders. (1) 1ml-Hamilton-Spritze; (2) Hartplastikstopfen; (3) Metallgehäuse; (4) Überwurfmutter; (5) Membran; (6) Ringdichtung. [80]

Für die Extrusion der Vesikel wird der manuelle Extruder Avestin LiposoFast Basic verwendet, dessen Querschnitt in Abb.5.1 dargestellt ist. Vor der Extrusion wird eine der Hamilton-Spritzen (1) mit der unextrudierten, aber gut durchmischten Suspension befüllt. Versuche zeigen, dass der Vorgang des Extrudierens wesentlich erleichtert wird, wenn die Suspension eine Temperatur oberhalb der Umwandlungstemperatur besitzt. Das Lipidgemisch in der Spritze wird langsam durch den Kanal im Hartplastikstopfen (2) und die Polycarbonatmembran (5) gedrückt. Die Polycarbonat-Membran, die ebenfalls von Avestin bezogen wurde, ist mit hexagonalen Gitter von Poren mit einem Durchmesser von $100 \mathrm{~nm}$ durchsetzt. Das Pressen der Lipidsuspension durch die Membran führt zu Abschnürungen in den multilamellaren Vesikeln. Dieser Prozess tritt besonders häufig während des ersten Extrudierens auf. Nach mehrmaliger Extrusion (empfohlen werden 15-21 Durchgänge durch die Membran) entstehen bei Verwendung eines $100 \mathrm{~nm}$-Filters unilamellare Vesikel von ungefähr $80 \mathrm{~nm}$ für DMPC. Die Größe der resultierenden Vesikel hängt aber nicht nur vom Porendurchmesser, sondern auch vom verwendeten Lipid ab [80]. Wird die Suspension ungeradzahlige Male durch die Membran gepresst, so befindet sich die Suspension in der „sauberen“ Spritze. Vor dem ersten Extrudieren können sich auf der Membranseite der zuerst befüllten Spritze Verunreinigungen und nicht extrudierte Vesikel sammeln. Durch Entnahme der Suspension auf der entgegengesetzten Seite verbleiben diese im Extruder. Es ist daher auch darauf zu achten, dass die Membran nach der Extrusion von 5-10 ml Suspension ausgetauscht wird. 


\section{Messergebnisse der Ultraschallspektrometrie}

\subsection{Die Spektren}

Die Spektren der Suspensionen von DMPC/Alamethicin-Membranen lassen sich, wie im Folgenden gezeigt wird, durch die gleichen Terme beschreiben wie die der reinen DMPCMembranen. In bisherigen Messungen [40, 98, 100] wurden Untersuchungen an Vesikellösungen mit einer Konzentration von $2 \mathrm{mg} / \mathrm{ml}$ DMPC und $10 \mathrm{mg} / \mathrm{ml}$ DMPC verwendet. Im Rahmen dieser Arbeit wurden jedoch Vesikellösungen mit einer Konzentration von $5 \mathrm{mg} / \mathrm{ml}$ DMPC/Alamethicin angesetzt. Messungen an Lösungen mit nur $2 \mathrm{mg} / \mathrm{ml}$ zeigten bei der Auswertung nur kleine Amplituden der beteiligten Prozesse, während die Verwendung einer Lösung mit 10 mg/ml Substanz im Hinblick auf den Preis des Alamethicins zu kostspielig ist.

\subsubsection{Reines DMPC}

Wie sich im Folgenden zeigen wird, werden die Spektren von reinem DMPC nur durch monomolekulare Prozesse beschrieben, daher sind die Relaxationszeiten unabhängig von der Vesikelkonzentration. Nach Gl. (3.15) geht die Konzentration der Vesikel linear in die Amplitude ein, so dass diese entsprechend der Konzentration skaliert werden kann.

Schrader et al. [100] beschreiben die Absorptionsspektren von reinem DMPC durch einen kritischen Fluktuationsterm nach Bhattacharjee und Ferrell und durch drei Einzelzeitrelaxationen, die der Rotationsdiffusion des Lipids, der Isomerisation der Alkylketten und einer lokalen Vesikeldeformation entsprechen.

\section{Axiale Diffusion}

In Tab. 6.1 sind die Parameter der unterschiedlichen Absorptionsbeiträge für reines DMPC aufgeführt. Die Einzelzeitrelaxation im Zeitbereich von $\tau=10-20$ ns, die mit der Amplitude $A_{\text {ax }}$ und der Relaxationszeit $\tau_{\text {ax }}$ beschrieben wird, entspricht der sogenannten axialen Diffusion, der Rotation um die Längsachse des Lipidmoleküls. Man erkennt eine leichte Verlangsamung in der Nähe der kritischen Temperatur $T_{\mathrm{c}}=24,0 \pm 0,1^{\circ} \mathrm{C}$ [100]. Während die Werte um $T_{\mathrm{c}}$ mit den Relaxationszeiten der lateralen Diffusion, also Bewegungen in der Membranebene, übereinstimmen, entsprechen sie in größerem Abstand von der 


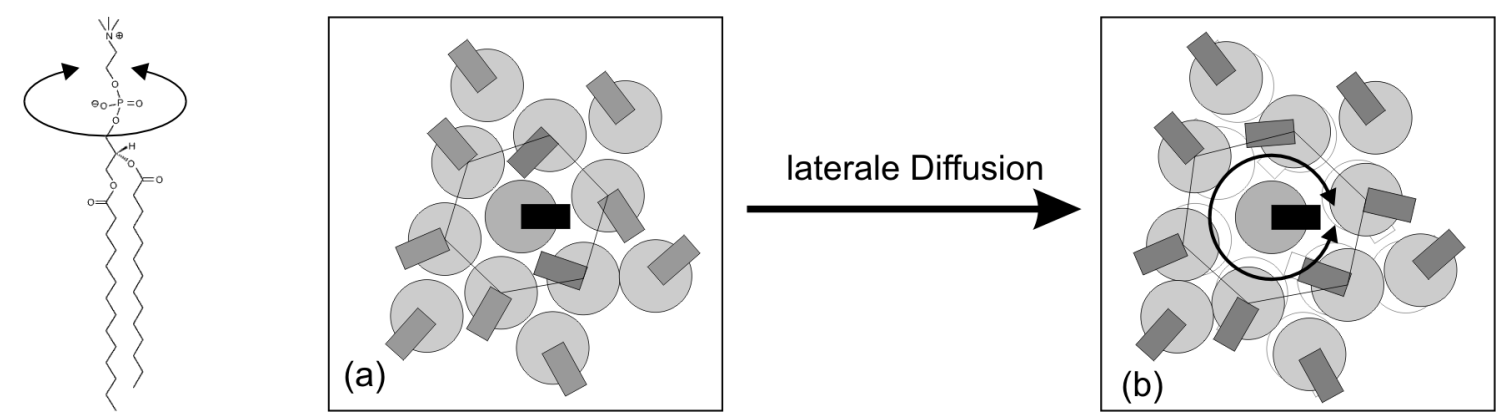

Abbildung 6.1: Rotation und laterale Diffusion. Diese Abbildung soll den Zusammenhang zwischen Rotationsdiffusion und lateraler Diffusion verdeutlichen. In (a) sind die Moleküle dicht gepackt, so dass das hervorgehobene unsymmetrische Lipid nicht frei rotieren kann. Wenn durch laterale Diffusion freie Volumina in der Membran entstehen, wie in (b) gezeigt, ist eine Rotation möglich.

kritischen Temperatur der Relaxationszeit der axialen Diffusion, wie NMR-Spektren deuterierter Lipide zeigen [103]. Unter Berücksichtigung des Critical Slowing Down, ist daher anzunehmen, dass es sich insgesamt um eine Rotation um die Längsachse handelt.

Bei der axialen Diffusion handelt es sich um einen kooperativen Prozess, an dem auch die Nachbarmoleküle des rotierenden Lipids beteiligt sind. Das Molekül weist eine Asymmetrie entlang der Längsachse auf, so dass es ein freies Volumen in unmittelbarer Nachbarschaft benötigt, wodurch die axiale Diffusion mit der lateralen Diffusion verknüpft ist (siehe auch Abb.6.1). Über deren Diffusionskonstante $D$ und Gl. (3.25) kann damit auch ein Zusammenhang mit dem Critical Slowing Down hergestellt werden.

\section{Rotationsisomerisation der Alkylketten}

Im oberen Frequenzbereich der Ultraschallspektren, entsprechend einer Relaxationsrate von $\tau \approx 0,1 \mathrm{~ns}$, kann das Spektrum durch eine Einzelzeitrelaxation mit der Amplitude $A_{\text {iso }}$ und der Relaxationszeit $\tau_{\text {iso }}$ angepasst werden, die die Kinetik der Alkylkettenisomerisation beschreibt. Die Relaxationszeiten in der fluiden Phase stimmen ebenfalls mit Ergebnissen der Ultraschallabsorptionsspektrometrie an $n$-Alkanen gleicher Kettenlänge überein [8]. In der gelförmigen Phase ist die Relaxation etwas verlangsamt, da hier die Beweglichkeit der Moleküle durch die dichtere Packung der Lipide beeinträchtigt ist.

Untersuchungen mit NMR-Methoden an deuterierten Membranlipiden bestätigen ebenfalls die Zuordnung zur Rotationsisomerisation der Alkylketten[103].

Die Rotationsisomerisation ist ein kollektiver Prozess, dessen Relaxationszeit von der Kettenlänge abhängt. Aufgrund des kollektiven Verhaltens wird der Übergang von der geordneten in die ungeordnete Phase der Ketten als Phasenübergang erster Ordnung angesehen [37]. Die Relaxationszeiten dieses Prozesses zeigen daher auch keine kritische Verlangsamung nahe $T_{\mathrm{c}}$. 


\section{Lokale Vesikeldeformation}

Im Bereich der kritischen Temperatur wird das Spektrum durch eine weitere, sehr langsame Einzelzeitrelaxation mit $A_{\text {ves }}$ und $\tau_{\text {ves }}$ beschrieben. Schrader et al. [100] fassen diesen Term und den Term der kritischen Fluktuationen zu der erweiterten Spektralfunktion

$$
S^{\#}(\omega)=S(\omega)+\frac{A_{\mathrm{ves}}\left(\omega \tau_{\mathrm{ves}}\right)}{1+\left(\omega \tau_{\mathrm{ves}}\right)^{2}}
$$

mit

$$
S(\omega) \text { : Spektralfunktion }
$$

$A_{\text {ves }}, \tau_{\text {ves }}:$ Amplitude und Relaxationszeit des zusätzlichen Relaxationsterms

zusammen. In [100] wird vorgeschlagen, dass dieser Term mit einer lokalen Vesikeldeformation zu interpretieren ist. Gestützt wird diese Annahme durch optische Messungen der Membranfluktuationen und der Biegesteife der Membran, die ein kritisches Verhalten des Biegemoduls und eine Vesikeldeformation entlang der optischen Achse aufzeigen [76].

Abb. 6.2 zeigt exemplarisch das Absorptionsspektrum einer DMPC:Alamethicin-Vesikelsuspension mit einem Alamethicingehalt von $0,3 \mathrm{~mol} \%$ bei $31,95^{\circ} \mathrm{C}$ im Frequenzbereich von $100 \mathrm{kHz}$ bis $1 \mathrm{GHz}$. Auf der Abszisse wurde die Exzessdämpfung (siehe Abschnitt 3.1) aufgetragen. Die Messdaten verschiedener Apparaturen werden in der Grafik durch unterschiedliche Symbole repräsentiert. An die Daten wurde die Spektralfunktion $S(\omega)$ angepasst, die sich als Summe aus der Relaxationszeitverteilung des kritischen Anteils nach Bhattacharjee und Ferrell und zwei Einzelzeitrelaxationen zusammensetzt, die in der Anpassung durch Debye-Spektralterme berücksichtigt werden. Der niederfrequente Debye-Spektralterm kann der axialen Diffusion zugeordnet werden, der hochfrequente der Alkylkettenisomerisation. In dem dargestellten Spektrum tritt der Debye-Spektralterm, der der lokalen Vesikeldeformation zugeordnet werden kann, nicht auf.

Alle gemessenen Spektren sind im Anhang A zu finden. 


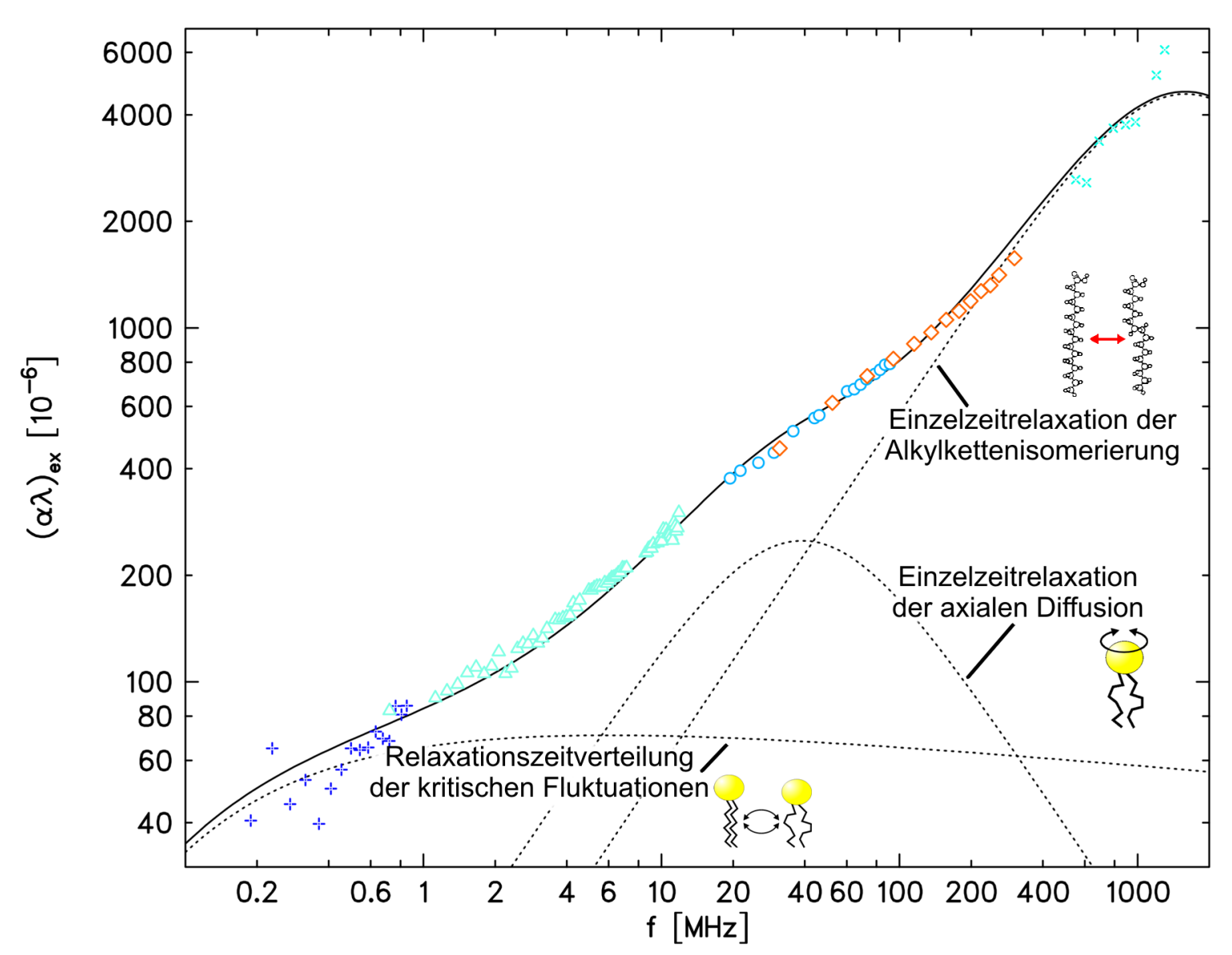

Abbildung 6.2: Interpretation eines Spektrums. In diesem Beispielspektrum (DMPC:Alamethicin, 99,7:0,3 mol\%, 31,95 ${ }^{\circ} \mathrm{C}$ ) sind die Beiträge der Relaxationszeitverteilung der kritischen Fluktuationen sowie die der Einzelzeitrelaxationen der axialen Diffusion und der Alkylkettenisomerierung gestrichelt dargestellt. Die durchgezogene Linie repräsentiert die Summe der einzelnen Beiträge. Der Beitrag aus dem Hintergrundanteil wurde bereits subtrahiert. Symbole für Apparaturen: +: 1-MHz-Reflektor-Resonator; $\triangle$ : 8-MHz-Resonator; $\circ$ : tieffrequente Pulszelle; $\diamond$ : hochfrequente Pulszelle; $\times$ : tieffrequente Hyperschallzelle. 
Tabelle 6.1: Messdaten für DMPC. Die Werte wurden [100] entnommen und die Amplituden unter Annahme einer linearen Konzentrationsabhängigkeit auf eine Konzentration von $5 \mathrm{mg} / \mathrm{ml}$ DMPC umgerechnet.

\begin{tabular}{|c|c|}
\hline$\sim \stackrel{n}{n} \frac{5}{7}$ & 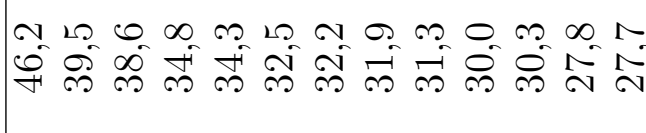 \\
\hline 总点 & 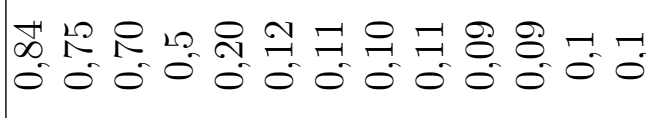 \\
\hline & 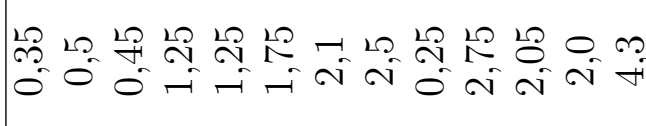 \\
\hline & 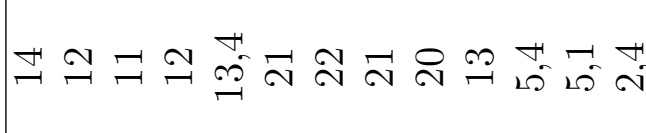 \\
\hline & 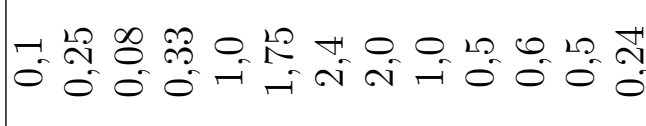 \\
\hline 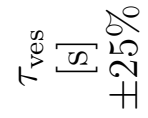 & 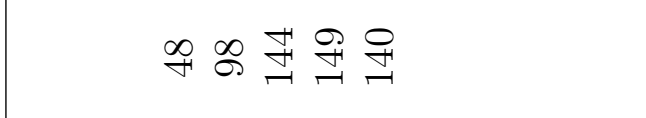 \\
\hline 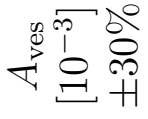 & 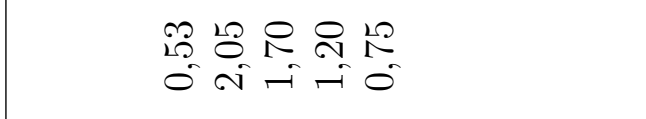 \\
\hline ケ范 & 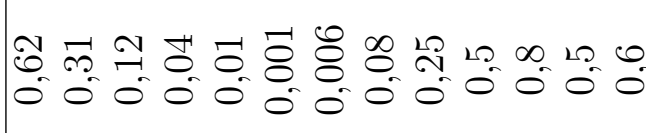 \\
\hline 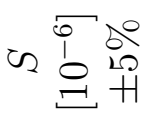 & 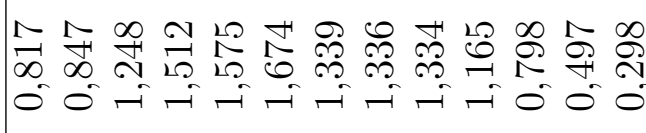 \\
\hline 心 & 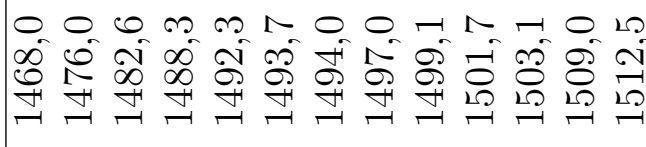 \\
\hline$-\underset{0}{0}$ & 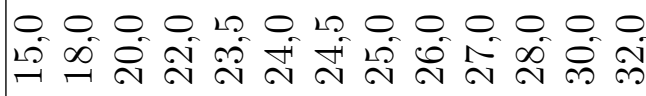 \\
\hline
\end{tabular}




\subsubsection{Messergebnisse der DMPC/Alamethicin-Suspensionen}

Während in vorherigen Arbeiten die Ultraschallabsorptionsspektrometrie hauptsächlich genutzt wurde, um die molekularen Prozesse innerhalb der Membran zu beschreiben, ist der Fokus dieser Arbeit auf den Einfluss von Alamethicin auf die kritischen Fluktuationen gerichtet.

Dazu wurden bis zu 13 Spektren einer DMPC/Alamethicin-Lösung bei verschiedenen Temperaturen ausgemessen. Um die starken Änderungen der Parameter nach einem Potenzgesetz in der Nähe der kritischen Temperatur zu berücksichtigen, wurde die Größe der Temperaturschritte in der Nähe von $T_{\mathrm{c}}$ verringert.

Alle Spektren wurden mit dem in Abschnitt 4.2.7 beschriebenen Aufbau im Frequenzbereich zwischen 0,1-12 MHz gemessen. Um die Messzeit der Spektren zu reduzieren, wurden komplette Spektren nur für jeweils zwei Temperaturen in der gelförmigen und der fluiden Phase aufgenommen, sowie ein Spektrum in der Nähe der kritischen Temperatur.

Die kompletten Spektren dienen in erster Linie zur Bestimmung der Hintergrunddämpfung. Da mit verdünnten wässrigen Lösungen gearbeitet wird, kann für die Interpolation der Hintergrunddämpfung eine empirische Näherung angenommen werden, die auf den experimentellen Dämpfungswerten von Wasser beruht:

$$
\begin{aligned}
\left(\alpha / f^{2}\right)_{\exp }(T) & \approx\left(51,712-1,9970 \cdot T+0,038806 \cdot T^{2}-0,0003045 \cdot T^{3}\right) \cdot 10^{-15} \\
B & =\left(\alpha / f^{2}\right)_{\exp } \cdot c_{\mathrm{s}} .
\end{aligned}
$$

Die Schallgeschwindigkeit $c_{\mathrm{s}}$ wird durch Velocimetrie bestimmt (siehe auch Abschnitt 4.4).

Zur Bestimmung der Relaxationsraten der Alkylkettenisomerisation sind Spektren notwendig, die auch den Frequenzbereich oberhalb $500 \mathrm{MHz}$ abdecken. Da die Isomerisation unabhängig von dem kritischen Verhalten des Systems ist, können die Relaxationszeiten und Amplituden des entsprechenden Terms des reinen DMPCs übernommen werden.

Die angegebenen Fehler wurden anhand der Messfehler von Schrader et al. [100] abgeschätzt. Bei der Anpassung der Daten konnten in der Nähe der kritischen Temperatur keine Fehler angegeben werden, da die Terme zur Bestimmung des kritischen Verhaltens „festgehalten" werden mussten.

Auf den folgenden Seiten sind tabellarisch die Parameter dargestellt, die zur Beschreibung der Spektren nötig sind. Die dazugehörigen Spektren sind im Anhang A zu finden. 


\section{DMPC:Alamethicin, 99,7:0,3 mol\%}

Tabelle 6.2: Messdaten für DMPC:Alamethicin, 99,7:0,3 mol\%. Die diesen Daten zu Grunde liegenden Spektren sind im Anhang A.1 abgebildet.

\begin{tabular}{|c|c|}
\hline ص & 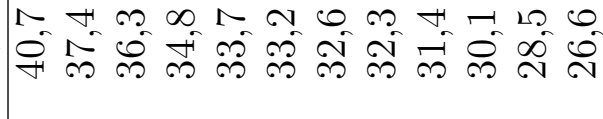 \\
\hline & 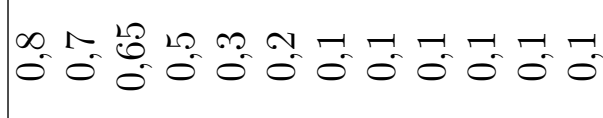 \\
\hline$\stackrel{0}{\infty}$ & 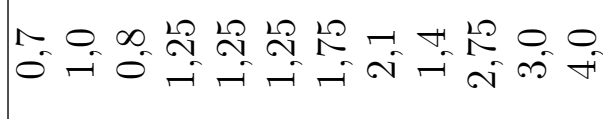 \\
\hline & 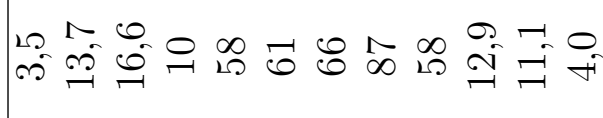 \\
\hline & 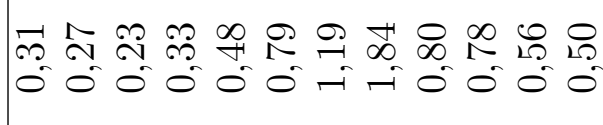 \\
\hline & 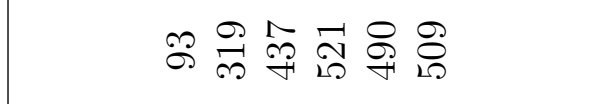 \\
\hline & 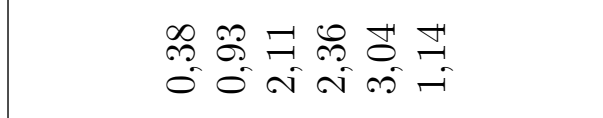 \\
\hline 千T & 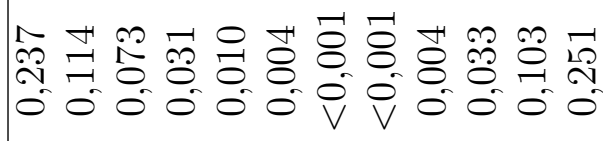 \\
\hline v & 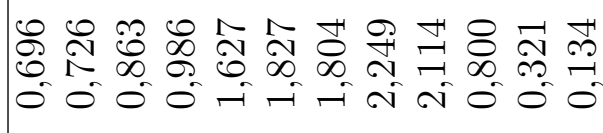 \\
\hline & 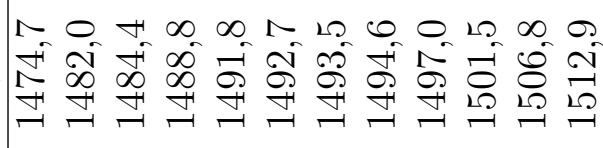 \\
\hline$+\underset{0}{\circlearrowright}$ & 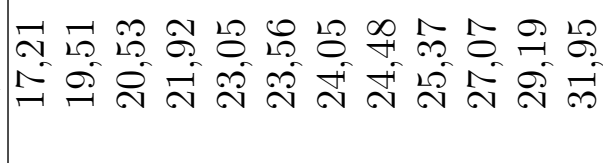 \\
\hline
\end{tabular}




\section{DMPC:Alamethicin, 99,5:0,5 mol\%}

Tabelle 6.3: Messdaten für DMPC:Alamethicin, 99,5:0,5 mol\%. Die diesen Daten zu Grunde liegenden Spektren sind im Anhang A.2 abgebildet.

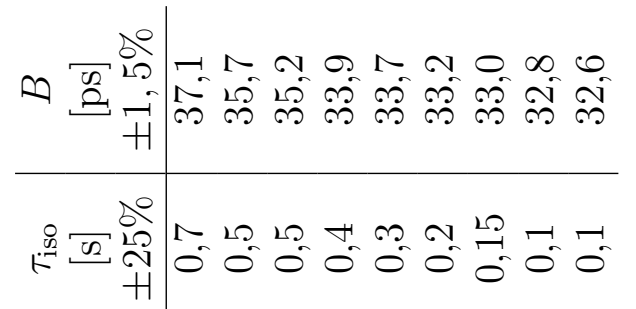

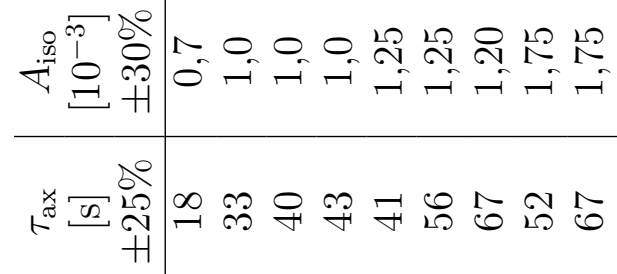

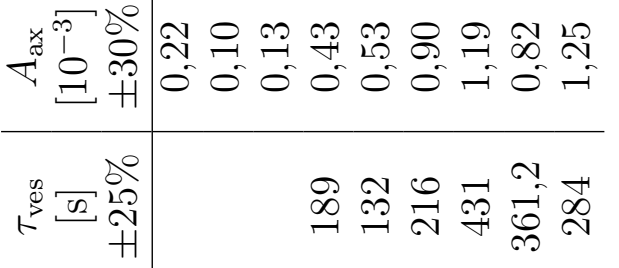

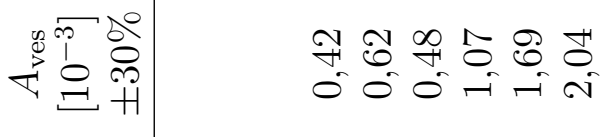

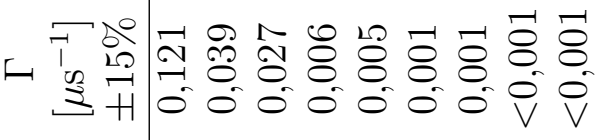

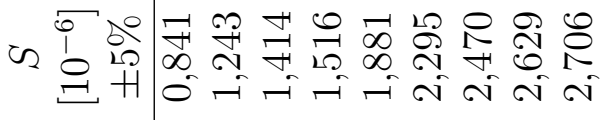

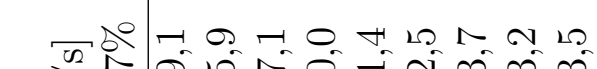

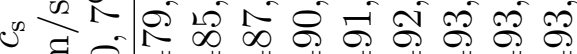

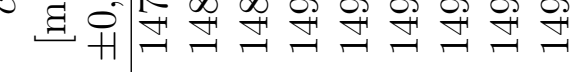

$$
\begin{aligned}
& \text { ช }
\end{aligned}
$$

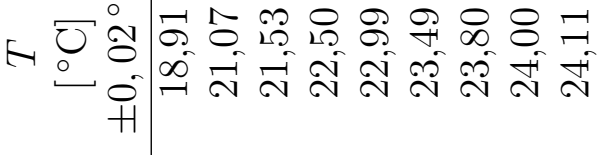




\section{DMPC:Alamethicin, 99,3:0,7 mol\%}

Tabelle 6.4: Messdaten für DMPC:Alamethicin, 99,3:0,7 mol\%. Die diesen Daten zu Grunde liegenden Spektren sind im Anhang A.3 abgebildet.

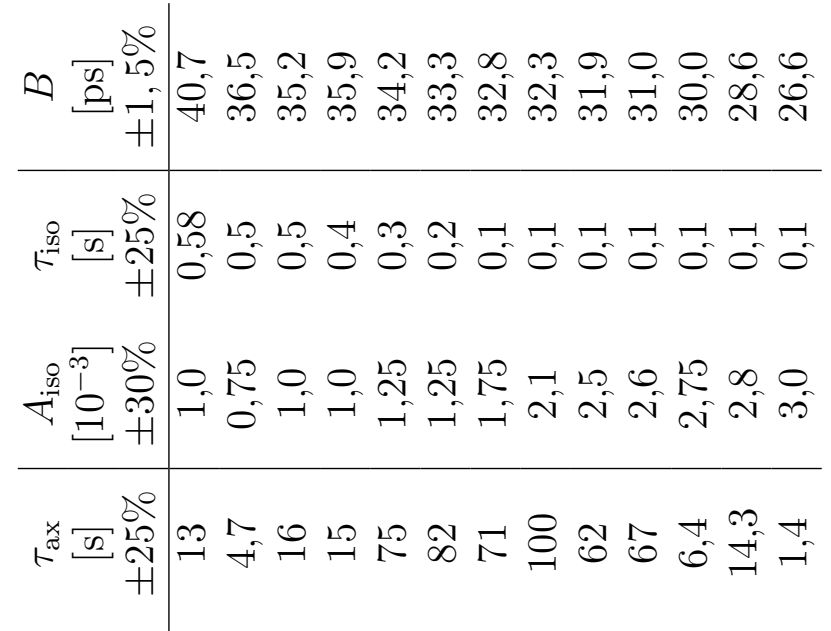

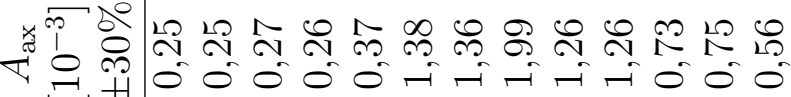

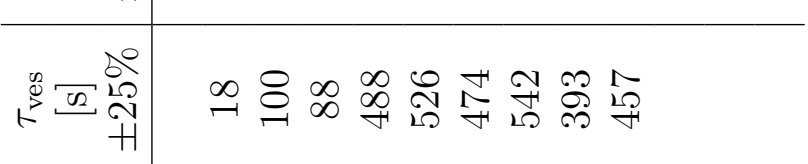

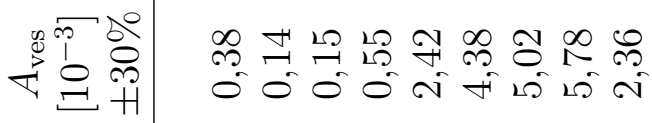

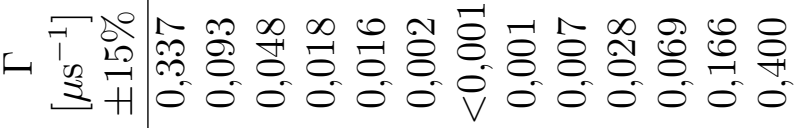

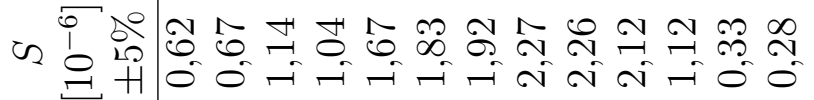

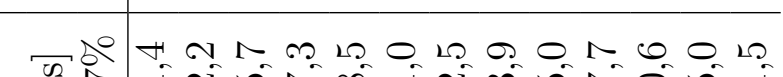

$$
\begin{aligned}
& \text { 以 }
\end{aligned}
$$

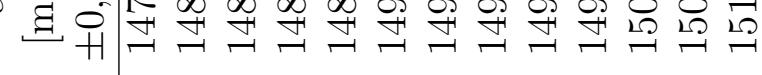

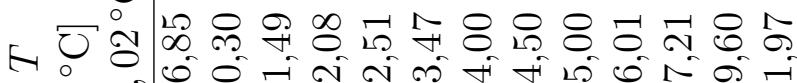

$$
\begin{aligned}
& \text { + }
\end{aligned}
$$




\section{DMPC:Alamethicin, 99:1 mol\%}

Tabelle 6.5: Messdaten für DMPC:Alamethicin, 99:1 mol\%. Die diesen Daten zu Grunde liegenden Spektren sind im Anhang A.4 abgebildet.

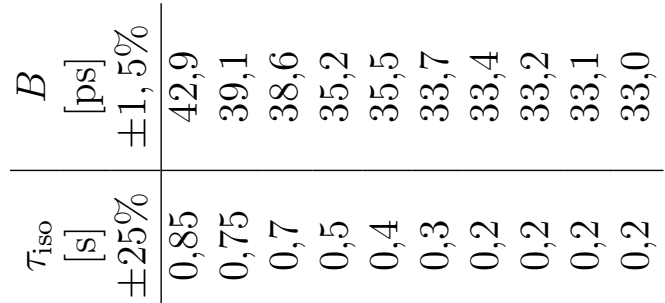

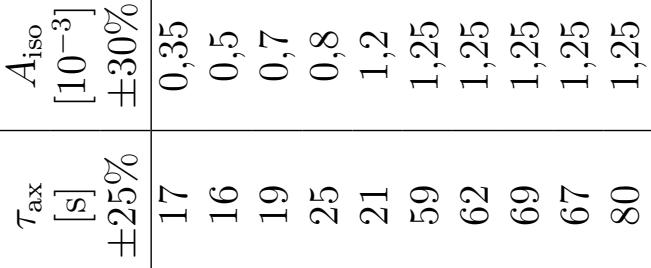

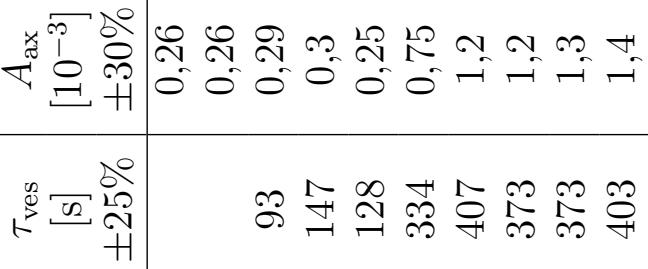

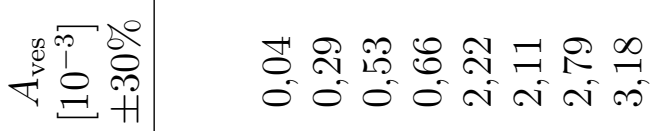

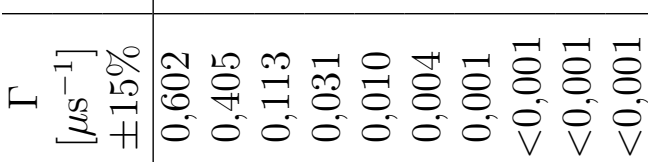

$$
\begin{aligned}
& \text { य }
\end{aligned}
$$

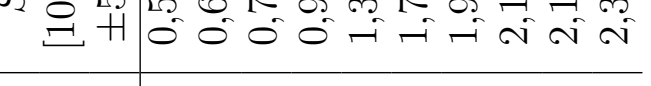

$$
\begin{aligned}
& \text { m }
\end{aligned}
$$

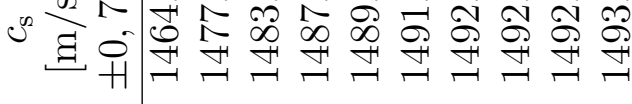

$$
\begin{aligned}
& \text { - }
\end{aligned}
$$

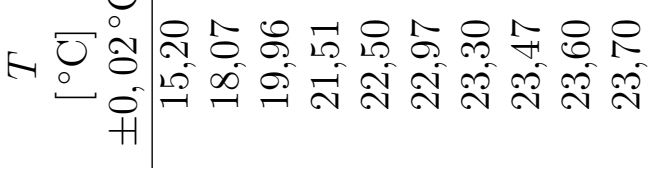




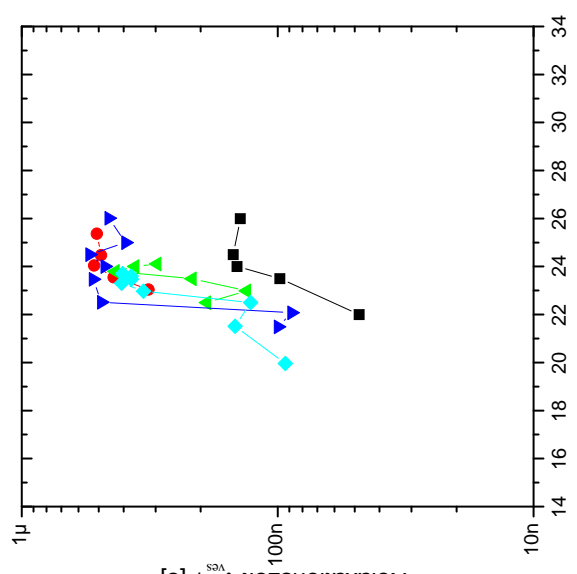

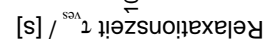

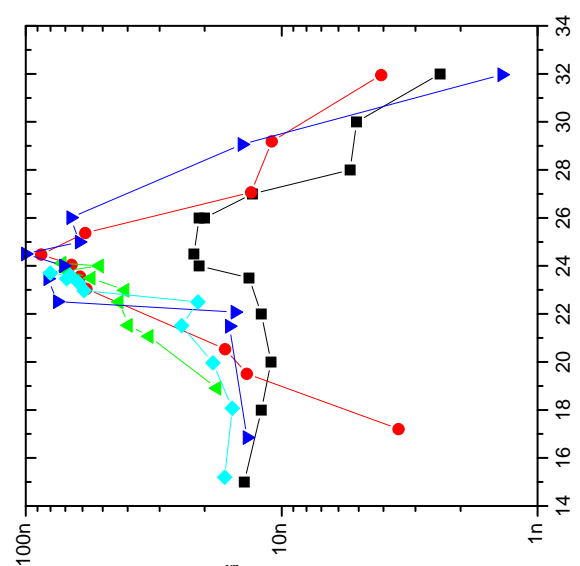

[s] $/{ }^{\mathrm{xp}} 2$ џ!əzsuo!̣exe

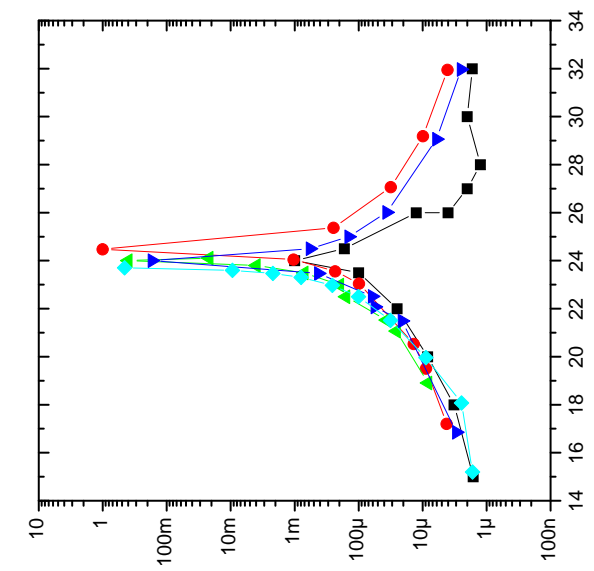

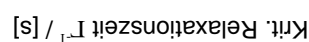
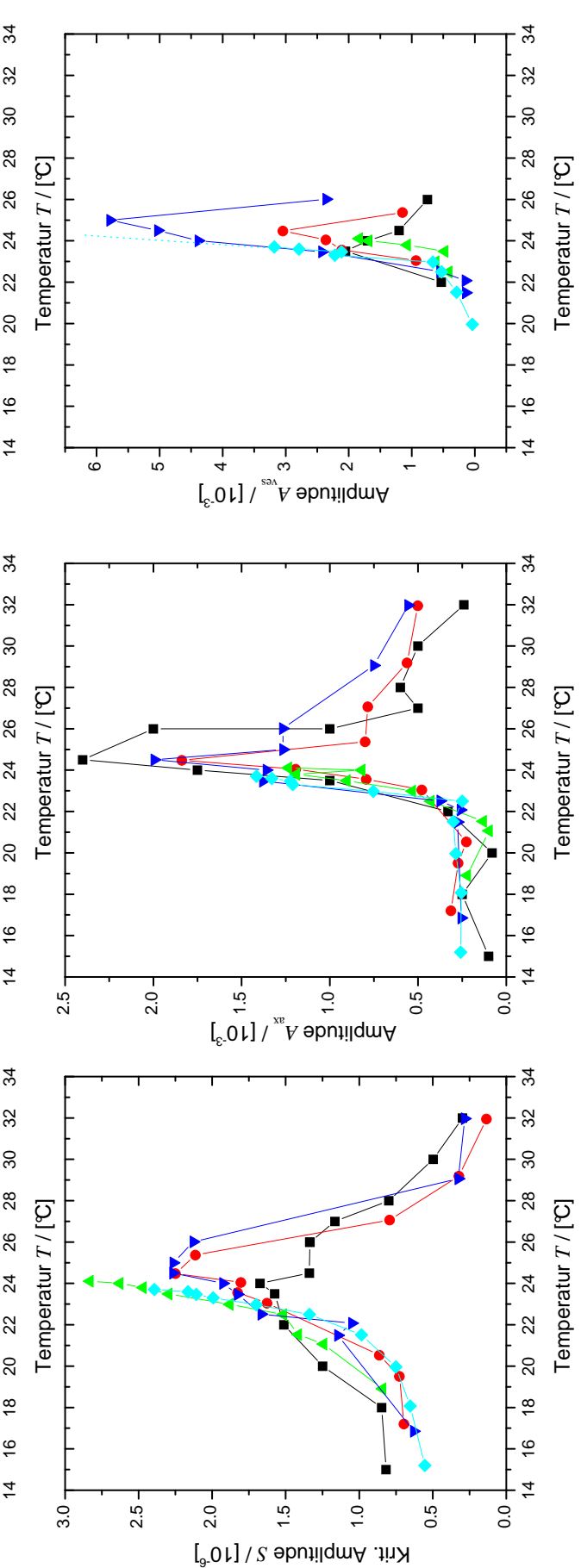

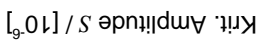

$\frac{0}{0} \stackrel{ \pm}{ \pm} \frac{0}{0} \varepsilon$

ס

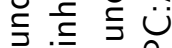

ร ํำ ำ

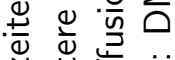

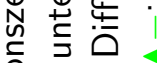

음 의

$\stackrel{\frac{\pi}{x}}{\frac{\pi}{\alpha}} \frac{\overline{0}}{\pi}$

$\stackrel{x}{\frac{0}{\omega}} \stackrel{\bar{\Delta}}{\frac{\pi}{x}} \frac{0}{0}$

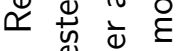

. d

ర

믐 음

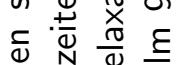

ป

은 은

ن

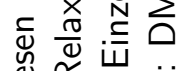
음 $\frac{1}{0} . \underline{0}$ $\leq$ 드 ०ं 들 है

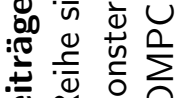
๑

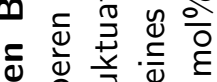

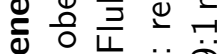
웜 ๖ें

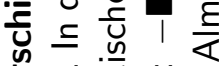
ঠ் 这芒 - $\frac{00}{\bar{N}}$ ⿹

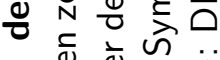

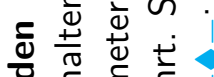

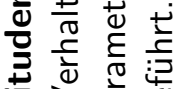

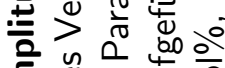

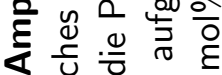

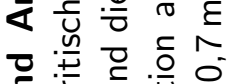
들 ธิ 늠

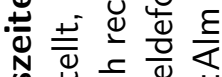
N .0

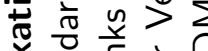
希

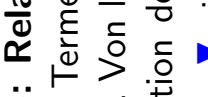
$\ddot{\bar{\nu}}$ ตั 일

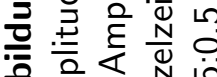
造完 $\frac{0}{0}$ i 


\section{DMPC:Alamethicin, 98:2 mol\%}

Es wurden ebenfalls Messungen an alamethicinhaltigen DMPC-Membranen mit einem Alamethicingehalt von $2 \mathrm{~mol} \%$ durchgeführt. Jedoch konnte in der Nähe der kritischen Temperatur die Präzipitation des Lipids beobachtet werden, welche zu einem Konzentrationgradienten innerhalb der Vesikellösung führt. Die Ultraschallspektrometrie setzt sowohl beim Resonator- als auch Schwingungspulstransmissionsverfahren eine homogene Lösung voraus. Aus diesem Grunde konnte für diese Alamethicinkonzentration keine konsistente Anpassung der Spektren durchgeführt werden.

Eine mögliche Erklärung für die Präzipitation wird mit der Vesikeldeformation in Abschnitt 6.2 .3 gegeben.

\subsection{Interpretation der Messergebnisse}

Messungen an DMPC-Membranen [40, 54, 98] zeigen, dass sich das Verhalten der kritischen Fluktuationen am Phasenübergang von der gelförmigen Phase in die fluide Phase durch eine empirische Skalierungsfunktion nach Bhattacharjee und Ferrell beschreiben lässt.

Es konnte auch gezeigt werden, dass diese Beschreibung für Lipidgemische [20, 43] und Membranen, die aus DMPC und Cholesterol bestehen [40, 54, 98], gültig ist. Cholesterolhaltige Membranen spielen bei der Interpretation der neuen Messergebnisse eine wichtige Rolle. Aus diesem Grunde soll an späterer Stelle ein kurzer Einschub über Lipid/Cholesterol-Membranen erfolgen.

\subsubsection{Die empirische Skalierungsfunktion}

\section{Quantitative Beschreibung der kritischen Parameter}

Ausgehend von vorherigen Messungen an Lipid- und Cholesterolgemischen kann erwartet werden, dass sich das Verhalten der Fluktuationen von DMPC/Alamethicin-Membransystemen durch einen kritischen Absorptionsterm nach Bhattacharjee und Ferrell beschreiben lässt durch Gl. (3.35)

$$
(\alpha \lambda)_{\mathrm{ex}}^{\mathrm{cr}}=A_{\mathrm{BF}}(T) F(\Omega) f^{-\tilde{\alpha}_{0} / z_{0} \tilde{\nu}},
$$

Mit Hilfe der empirischen Skalierungsfunktion nach Gl. (3.31)

$$
F(\Omega)=\frac{(\alpha \lambda)_{\mathrm{ex}}^{\mathrm{cr}}(T)}{(\alpha \lambda)_{\mathrm{ex}}^{\mathrm{cr}}\left(T_{\mathrm{c}}\right)}
$$

lässt sich überprüfen, ob der kritische Anteil in den Spektren nach dem Modell von Bhattacharjee und Ferrell angepasst werden kann. Dazu wird ein Datensatz in unmittelbarer Nähe der kritischen Temperatur $T_{\mathrm{c}}$ als Bezug gewählt. Nach dem FluktuationsDissipations-Gesetz [49] ist hier mit einem Maximum der kritischen Amplitude zu rechnen, 


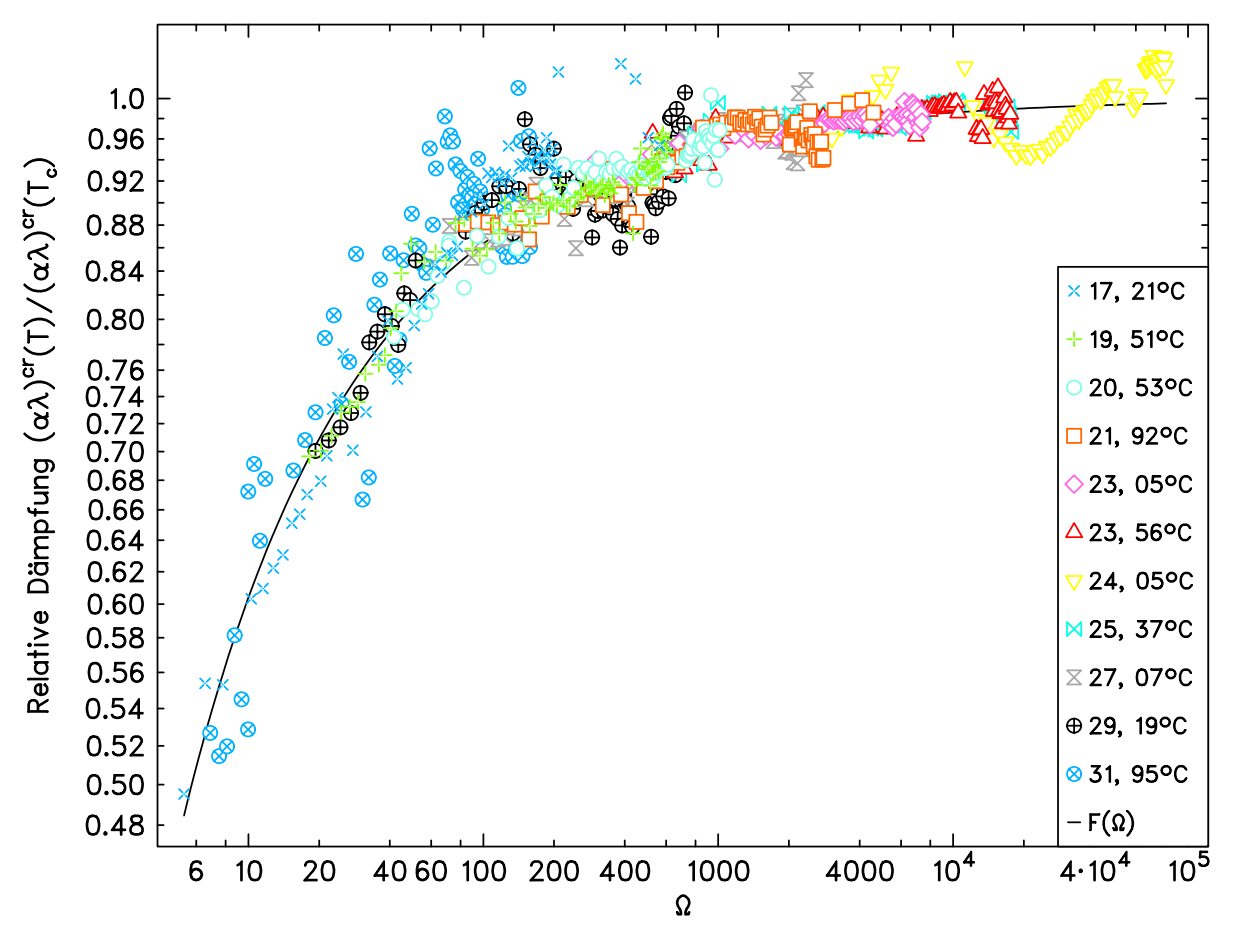

DMPC:Alamethicin, 99,7:0,3 mol\%

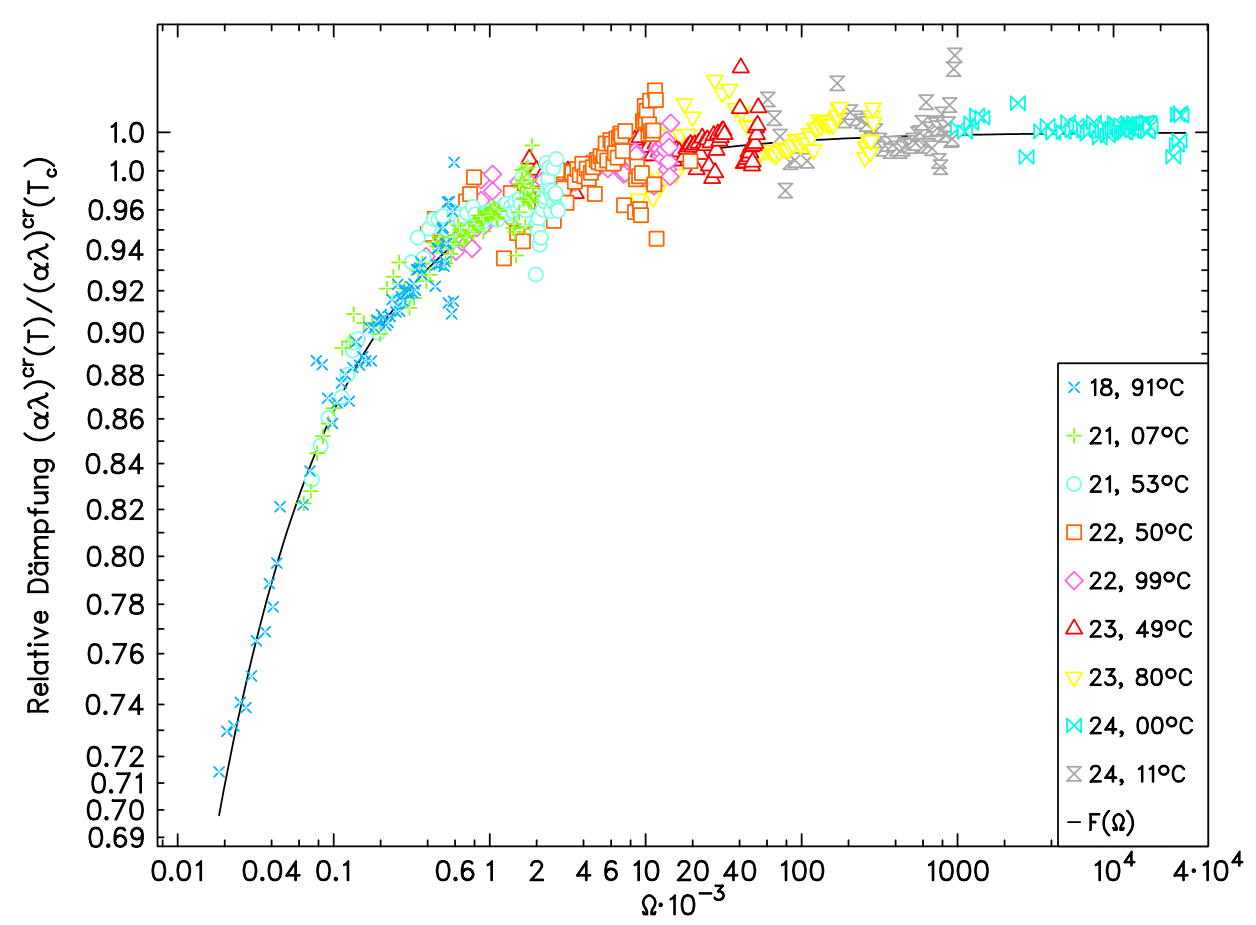

DMPC:Alamethicin, 99,5:0,5 mol\%

Abbildung 6.4: Empirische Skalierungsfunktionen. Darstellung der empirischen Skalierungsfunktionen bei verschiedenen Alamethicinkonzentrationen. 


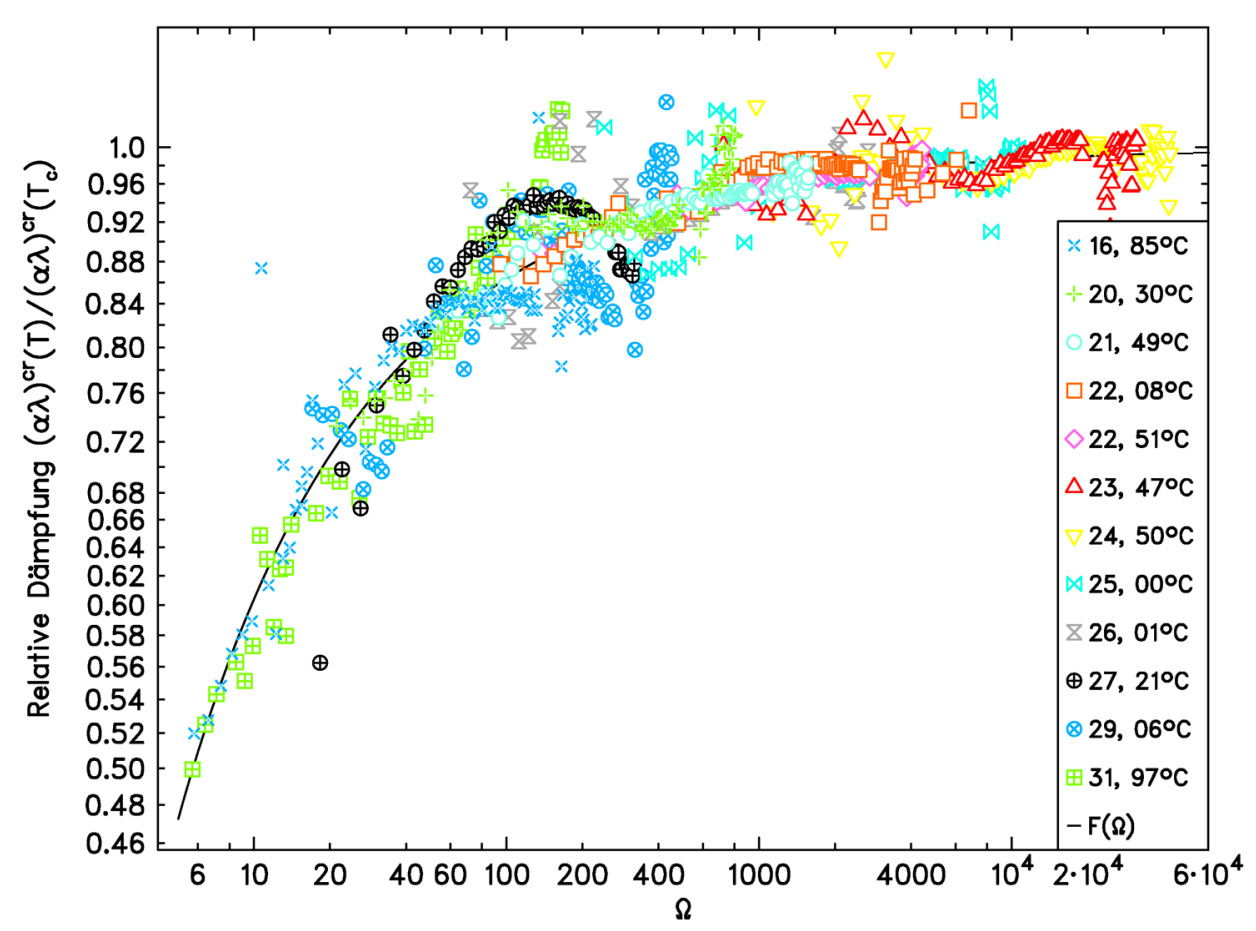

DMPC:Alamethicin,99,3:0,7 mol\%

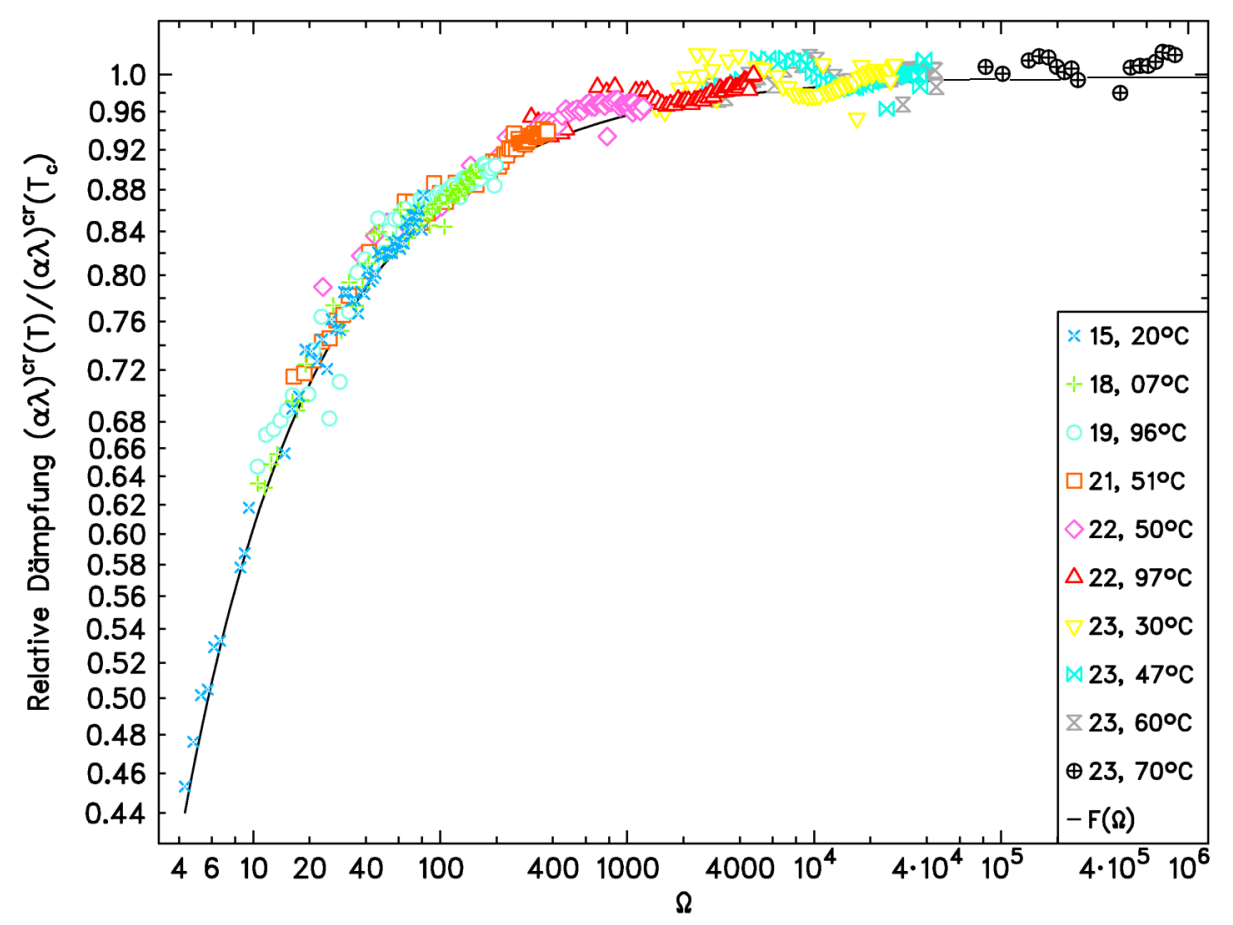

DMPC:Alamethicin, 99:1 mol\%

Abbildung 6.5: Empirische Skalierungsfunktionen. Darstellung der empirischen Skalierungsfunktionen bei verschiedenen Alamethicinkonzentrationen. 
so dass die kritische Temperatur anhand der maximalen Amplitude abgeschätzt werden kann.

Der kritische Anteil ergibt sich aus der Anpassung der Messdaten

$$
(\alpha \lambda)_{\mathrm{ex}}^{\mathrm{cr}}=(\alpha \lambda)-\sum_{n}\left(A_{n} \frac{\omega \tau_{n}}{1+\left(\omega \tau_{n}\right)^{2}}\right)-B f
$$

mit

$(\alpha \lambda)_{\mathrm{ex}}^{\mathrm{cr}}$ : kritischer Anteil der Ultraschallabsorption

$(\alpha \lambda)$ : gemessene Gesamtabsorption

$A_{n}, \tau_{n}$ : Amplitude und Relaxationszeit auftretender Einzelzeitrelaxationen

$B$ : Hintergrunddämpfung.

Die Abbildungen 6.4 und 6.5 zeigen eine gute Übereinstimmung der empirischen Skalierungsfunktion nach Gl. (3.31) mit der vorhergesagten Skalierungsfunktion für den flüssigkristallinen Phasenübergang nach Gl. (3.33), wenn lediglich die Relaxationsrate als anzupassender Parameter betrachtet wird.

Alamethicin bewirkt eine Reduktion der kritischen Relaxationsrate $\Gamma$ in der Nähe der kritischen Temperatur. Gleichzeitig tritt an der Umwandlungstemperatur ein Debye-Spektralterm mit hoher Amplitude auf, der ebenso wie die Relaxationsrate an der unteren Grenze des Messbereiches angesiedelt ist.

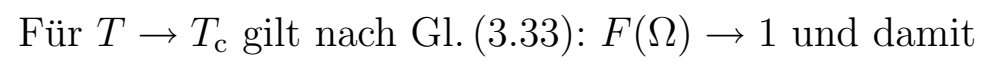

$$
(\alpha \lambda)_{\mathrm{ex}} \approx A_{\mathrm{BF}} \cdot f^{-0,06} .
$$

Die Funktion weist damit nur eine sehr geringe Frequenzabhängigkeit auf. Die Bestimmung der Relaxationsrate $\Gamma$ ist bei Annäherung an den kritischen Punkt nur noch mit einem sehr großen Fehler möglich. Der verwendete Marquardt-Algorithmus [94] konvergiert für Relaxationsraten $\Gamma<50 \cdot 10^{3} \mathrm{~s}^{-1}$ nicht mehr und verringert die Relaxationsrate kontinuierlich bei gleichzeitiger Erhöhung der zugehörigen Amplitude.

In den vorliegenden Messungen wurde das Problem durch Vorgabe und „Festhalten“ der Relaxationsrate $\Gamma$ für Messungen in der Nähe der kritischen Temperatur gelöst. Zur Bestimmung von $\Gamma$ wurde die Annahme verwendet, dass die dynamische Skalierungstheorie gültig ist und $\Gamma$ nach Gleichung Gl. (3.25)

$$
\Gamma=\frac{2 D}{\xi^{2}}=\Gamma_{0}\left|\frac{T-T_{\mathrm{c}}}{T_{\mathrm{c}}}\right|^{-z_{0} \tilde{\nu}}
$$

anhand vorliegender Messdaten extrapoliert werden kann. Umgekehrt lässt sich aus dieser Gleichung die kritische Temperatur $T_{\mathrm{c}}$, sowie die charakteristische Relaxationsrate $\Gamma_{0}$ abschätzen, die als Maß für die Relaxationsgeschwindigkeit angenommen werden kann. Es ergeben sich die in Tab. 6.6 dargestellten Werte.

Eine Angabe des Fehlers ist nicht sinnvoll, da für die Anpassung der Kurve zum Teil extrapolierte Relaxationsraten verwendet wurden. 
Tabelle 6.6: Verlauf der Relaxationsraten in Abhängigkeit von der Temperatur. Da sich im Allgemeinen das Verhalten in gelförmiger und fluider Phase unterscheidet, sind jeweils die Ergebnisse der Anpassung für Messreihen unter- und oberhalb (Indizes $<$ bzw. $>$ ) der Umwandlungstemperatur angegeben.

\begin{tabular}{|l||c|c||c|c|}
\hline & $\begin{array}{c}T_{\mathrm{c},<} \\
{\left[{ }^{\circ} \mathrm{C}\right]}\end{array}$ & $\begin{array}{c}\Gamma_{0,<} \\
{\left[\mathrm{s}^{-1} \mathrm{~K}^{-1}\right]}\end{array}$ & $\begin{array}{c}T_{\mathrm{c},>} \\
{\left[{ }^{\circ} \mathrm{C}\right]}\end{array}$ & $\begin{array}{c}\Gamma_{0,>} \\
{\left[\mathrm{s}^{-1} \mathrm{~K}^{-1}\right]}\end{array}$ \\
\hline Reines DMPC & 24,31 & $0,48 \cdot 10^{9}$ & 24,19 & $1,1 \cdot 10^{9}$ \\
DMPC:Alm, 99,7:0,3 mol\% & 24,46 & $0,29 \cdot 10^{9}$ & 24,47 & $0,32 \cdot 10^{9}$ \\
DMPC:Alm, 99,5:0,5 mol\% & 24,02 & $0,25 \cdot 10^{9}$ & & \\
DMPC:Alm, 99,3:0,7 mol\% & 24,02 & $0,39 \cdot 10^{9}$ & 24,09 & $0,54 \cdot 10^{9}$ \\
DMPC:Alm, 99:1 mol\% & 23,71 & $0,44 \cdot 10^{9}$ & & \\
\hline
\end{tabular}

Die Tabelle zeigt, dass sich die Relaxation unterhalb der Umwandlungstemperatur geringfügig verlangsamt. Große Abweichungen von der charakteristischen Relaxationsrate des reinen DMPC sind in der fluiden Phase zu finden. Jedoch differieren die Messdaten (Tab. 6.1) sehr stark von der durch die für reines DMPC gegebenen Parameter.

Nach Gl. (3.25) kann bei Kenntnis des Diffusionskoeffizienten $D$ die Korrelationslänge $\xi$ berechnet werden. In [100] werden die Diffusionskoeffizienten für die DMPC-Moleküle in der gelförmigen und in der fluiden Phase angegeben als

$$
\begin{aligned}
D_{\text {gel }} & =3 \cdot 10^{-11} \mathrm{~cm}^{2} \mathrm{~s}^{-1} \\
D_{\mathrm{fl}} & =7 \cdot 10^{-9} \mathrm{~cm}^{2} \mathrm{~s}^{-1} .
\end{aligned}
$$

Die Zahlenwerte berücksichtigen nicht das kritische Verhalten der Diffusion, ermöglichen jedoch eine Abschätzung der Korrelationslängen, anhand derer das Einsetzen des kooperativen Verhaltens bestimmt werden kann. Für Temperaturen weit entfernt von der kritischen Temperatur sind die Korrelationslängen wesentlich kleiner als die Abstände zwischen den Lipiden, die mit $d_{\mathrm{nn}, \mathrm{gel}}=0,38 \mathrm{~nm}$ in der Gelphase und $d_{\mathrm{nn}, \mathrm{fl}}=0,45 \mathrm{~nm}$ in der fluiden Phase angegeben werden. Ein korreliertes Verhalten der Fluktuationen kann erst einsetzen, wenn die Korrelationslänge größer ist als der Abstand zwischen den Lipidmolekülen. In Tab. 6.7 sind die Temperaturen hervorgehoben, für die eine Korrelation angenommen werden kann.

Tabelle 6.7: Berechnete Korrelationslängen. Die Tabelle enthält die berechneten Korrelationslängen nach GI. (3.25). Die fett gedruckten Werte symbolisieren Korrelationslängen $\xi>d_{\mathrm{nn}}$.

\begin{tabular}{|l|c||c|c|c|c|c|c|c|}
\hline \multirow{2}{*}{ Reines DMPC } & $T /\left[{ }^{\circ} \mathrm{C}\right]$ & 22,0 & $\mathbf{2 3 , 5}$ & $\mathbf{2 4 , 0}$ & $\mathbf{2 4 , 5}$ & $\mathbf{2 5 , 0}$ & $\mathbf{2 6 , 0}$ & $\mathbf{2 8 , 0}$ \\
\cline { 2 - 9 } & $\xi /[\mathrm{nm}]$ & 0,40 & $\mathbf{0 , 7 7}$ & $\mathbf{2 , 5}$ & $\mathbf{1 5}$ & $\mathbf{4 , 2}$ & $\mathbf{2 , 4}$ & $\mathbf{1 , 7}$ \\
\hline \hline \multirow{2}{*}{$\begin{array}{l}\text { DMPC:Alm, } \\
\text { 99,7:0,3 mol } \%\end{array}$} & $T /\left[{ }^{\circ} \mathrm{C}\right]$ & 21,92 & $\mathbf{2 3 , 0 5}$ & $\mathbf{2 4 , 0 5}$ & $\mathbf{2 4 , 4 8}$ & $\mathbf{2 5 , 3 7}$ & $\mathbf{2 7 , 0 7}$ & $\mathbf{2 9 , 1 9}$ \\
\cline { 2 - 9 } & $\xi /[\mathrm{nm}]$ & 0,44 & $\mathbf{0 , 7 6}$ & $\mathbf{2 , 5}$ & $\mathbf{1 8 3}$ & $\mathbf{1 9}$ & $\mathbf{6 , 6}$ & $\mathbf{3 , 7}$ \\
\hline \hline DMPC:Alm, & $T /\left[{ }^{\circ} \mathrm{C}\right]$ & 21,53 & $\mathbf{2 2 , 5 0}$ & $\mathbf{2 3 , 8 0}$ & $\mathbf{2 4 , 1 1}$ & & & \\
99,5:0,5 mol $\%$ & $\xi /[\mathrm{nm}]$ & 0,47 & $\mathbf{0 , 9 8}$ & $\mathbf{4 , 9}$ & $\mathbf{7 7}$ & & & \\
\hline \hline DMPC:Alm, & $T /\left[{ }^{\circ} \mathrm{C}\right]$ & 21,49 & $\mathbf{2 2 , 0 8}$ & $\mathbf{2 3 , 4 7}$ & $\mathbf{2 4 , 0 0}$ & $\mathbf{2 4 , 5 0}$ & $\mathbf{2 5 , 0 0}$ & $\mathbf{2 7 , 2 1}$ \\
\cline { 2 - 9 } $99,7: 0,3 \mathrm{~mol} \%$ & $\xi /[\mathrm{nm}]$ & 0,35 & $\mathbf{0 , 5 8}$ & $\mathbf{1 , 6}$ & $\mathbf{3 2}$ & $\mathbf{2 8}$ & $\mathbf{1 4}$ & $\mathbf{4 , 5}$ \\
\hline \hline DMPC:Alm, & $T /\left[{ }^{\circ} \mathrm{C}\right]$ & 21,51 & $\mathbf{2 2 , 5 0}$ & $\mathbf{2 3 , 4 7}$ & $\mathbf{2 3 , 7 0}$ & & & \\
99:1 mol $\%$ & $\xi /[\mathrm{nm}]$ & 0,44 & $\mathbf{0 , 7 8}$ & $\mathbf{3 , 6}$ & $\mathbf{5 2}$ & & & \\
\hline
\end{tabular}


Es zeigt sich, dass in der gelförmigen Phase die „Korrelationsgrenze“ in Anwesenheit von Alamethicin zu niedrigeren Temperaturen hin verschoben wird. Gleichbedeutend damit ist eine Erweiterung des Temperaturbereiches, in dem kritisches Verhalten zu erwarten ist.

Wie man aus dieser Abschätzung sieht, erstrecken sich die Messungen über einen Temperaturbereich, der weit über die Temperaturen hinaus geht, bei denen rein kritisches Verhalten zu erwarten ist.

Dieser so genannte Crossover-Bereich, stellt einen Überlapp der klassischen Beschreibung und der kritischen Beschreibung eines Fluids dar. Im Rahmen der Theorie von Bhattacharjee und Ferrell werden die Relaxationsraten $\Gamma$ durch die folgende Beziehung korrigiert (Crossover-Korrektur) [2, 16, 95]:

$$
\Gamma_{\mathrm{xo}}=\Gamma\left(1+\frac{1,18}{\xi \cdot q_{\mathrm{c}}}\right)^{z_{0} \tilde{\nu} / 2},
$$

mit

$$
\begin{gathered}
\xi: \text { Korrelationslänge } \\
q_{\mathrm{c}}: \text { Crossover-Wellenlänge. }
\end{gathered}
$$

\section{Vergleich mit Cholesterol}

Der Parameterverlauf der kritischen Amplitude und der kritischen Relaxationsrate der DMPC/Alamethicin-Suspension lässt sich mit dem Verhalten von DMPC/CholesterolSuspensionen vergleichen.
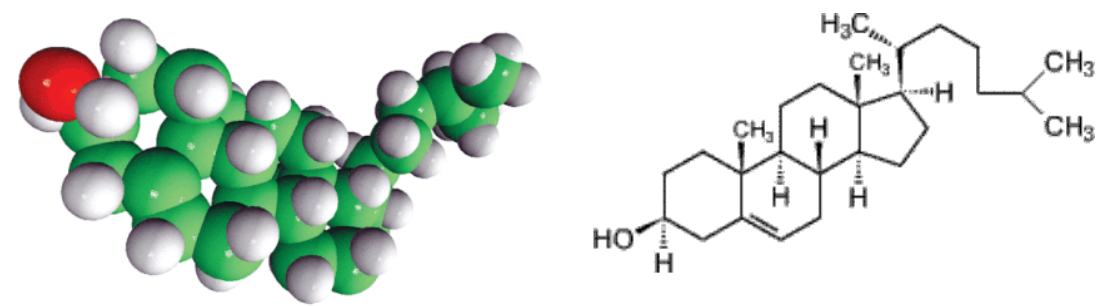

Abbildung 6.6: Struktur des Cholesterolmoleküls. Cholesterol besitzt einen hydrophoben Steroidkern, von dem eine ebenfalls hydrophobe Seitenkette und eine polare OH-Gruppe abzweigen [4].

Cholesterol ist ein Steroid, das als Regulator der Membranfluidität in fast allen biologischen Membranen enthalten ist [77] (siehe auch Abb. 2.1). Es lagert sich in den hydrophoben Alkylketten der Membran ein. Dabei geht die OH-Gruppe des Cholesterols mit der Glycerolgruppe eines Phospholipids eine Wasserstoffbrückenbindung ein. Die hydrophobe Ringstruktur des Cholesterols, dargestellt in Abb.6.6, führt zu einer Stabilisierung der Lipidketten in der geordneten all-trans-Konfiguration [34].

In Tab. 6.8 sind Messergebnisse von cholesterolhaltigen DMPC-Membranen mit verschiedenen Cholesterolgehalten $c_{\text {chol }}$ zusammengefasst [6]. 
Tabelle 6.8: Messwerte für cholesterolhaltige DMPC-Membranen. Die Tabelle enthält die Daten der Parameterverläufe für die kritische Fluktuation (Spalten $S$ und $\Gamma$ ), die Vesikeldeformation $\left(A_{\mathrm{ves}}, \tau_{\mathrm{ves}}\right)$ und die axiale Rotation $\left(A_{\mathrm{ax}}, \tau_{\mathrm{ax}}\right)$ für verschiedene Molenverhältnissen $c_{\mathrm{chol}}$ [6]. Die Amplituden wurden auf eine Gesamtkonzentration von $c_{\text {ges }}=5 \mathrm{mg} / \mathrm{ml}$ umgerechnet.

\begin{tabular}{|c|c|c|c|c|c|c|c|c|c|c|c|c|c|}
\hline$\infty$ & $\begin{array}{l}\widehat{O} \\
0 \\
0 \\
\infty \\
\infty \\
\infty\end{array}$ & $\begin{array}{l}\infty \\
20 \\
\infty \\
\infty\end{array}$ & $\begin{array}{l}\approx \\
20 \\
20 \\
\sim \\
\approx \\
\approx\end{array}$ & $\frac{\infty}{\infty}$ & 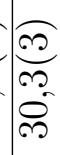 & 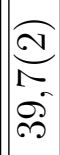 & 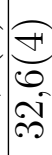 & 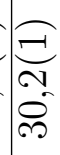 & 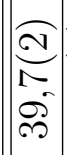 & 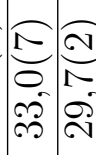 & $\left|\begin{array}{|l}\widehat{F} \\
\sigma \\
\infty \\
\infty \\
\infty\end{array}\right|$ & 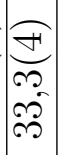 & $\begin{array}{l}0 \\
20 \\
20 \\
2\end{array}$ \\
\hline & $\stackrel{\overbrace{}}{\rightleftharpoons}$ & $\overparen{m}$ & 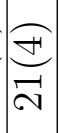 & $\frac{\overparen{Z}}{\vec{H}}$ & $\frac{10}{20}$ & $\frac{\Omega}{10}$ & $\overbrace{0}^{\infty}$ & 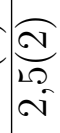 & 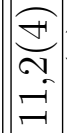 & 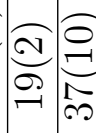 & 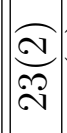 & 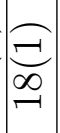 & $\stackrel{-}{-}$ \\
\hline & $\mid \begin{array}{l}0 \\
0 \\
0 \\
0 \\
0 \\
0\end{array}$ & 命 & $\begin{array}{l}10 \\
\infty \\
\infty \\
-i \\
-1\end{array}$ & $\begin{array}{l}100 \\
0 \\
0 \\
\text { in }\end{array}$ & $\frac{10}{20}$ & 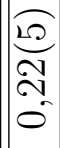 & 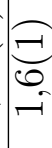 & $\stackrel{-}{-}$ & $\mid \begin{array}{c}2 \\
2 \\
20 \\
0 \\
0 \\
0\end{array}$ & 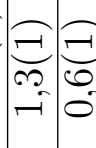 & 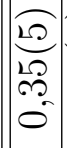 & $\begin{array}{l}120 \\
20 \\
\infty \\
0 \\
0\end{array}$ & 15 \\
\hline & & & 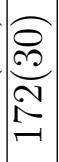 & $\frac{2}{2}$ & 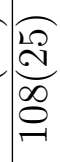 & & 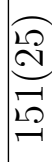 & 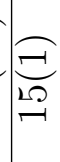 & & 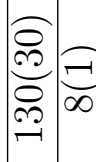 & & 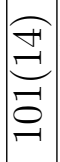 & $\approx$ \\
\hline & & $\frac{\widehat{O}}{\tilde{N}}$ & $\begin{array}{l}\tilde{O} \\
\stackrel{N}{N} \\
-i\end{array}$ & $\stackrel{\widetilde{N}}{\infty}$ & $\frac{\widetilde{\widetilde{N}}}{\tilde{\sigma}}$ & & 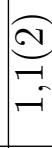 & $\stackrel{\vec{E}}{\sigma}$ & & 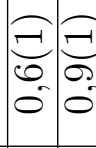 & & $\mid \begin{array}{c}10 \\
20 \\
0 \\
0 \\
0\end{array}$ & $\mid \begin{array}{l}10 \\
20 \\
\infty \\
0 \\
0\end{array}$ \\
\hline & $\underset{\tilde{\sigma}}{\stackrel{\rightleftharpoons}{\rightleftharpoons}}$ & 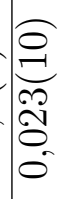 & 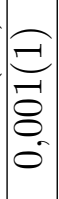 & 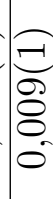 & $\underset{F}{\stackrel{E}{F}}$ & 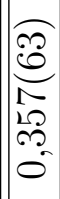 & $\begin{array}{l}\nexists \\
\\
8 \\
0 \\
0\end{array}$ & 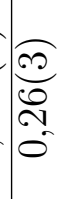 & 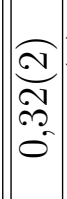 & 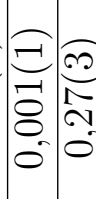 & 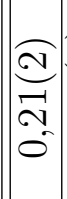 & $\mid \begin{array}{c}\mid \bar{\Xi} \\
\overline{8} \\
8 \\
0\end{array}$ & 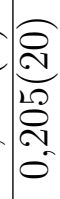 \\
\hline is & $\underset{\tilde{\theta}}{\tilde{\theta}}$ & $\frac{\widehat{D}}{\frac{\mathcal{O}}{20}}$ & $\underset{\widetilde{\sigma}}{\widetilde{\sigma}}$ & ֶָ & $\frac{\pi}{20}$ & $\frac{10}{10}$ & $\underset{\mathcal{E}}{\mathcal{E}}$ & $\frac{\tilde{E}}{\sigma}$ & $\mid \begin{array}{c}0 \\
0 \\
0 \\
-i \\
-i\end{array}$ & 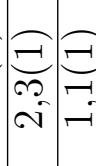 & $\mid$ & $\mid \begin{array}{l}10 \\
1 \\
\infty \\
-1 \\
-1\end{array}$ & 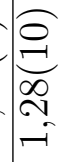 \\
\hline & $\begin{array}{l}0 \\
1 \\
\infty \\
\infty \\
+1 \\
-1\end{array}$ & 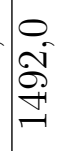 & 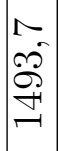 & $\begin{array}{l}0 \\
0 \\
\stackrel{5}{9} \\
\stackrel{+}{-}\end{array}$ & $\begin{array}{l}1 \\
0 \\
20 \\
10 \\
-1\end{array}$ & $\begin{array}{l}\sim \\
\mathscr{\infty} \\
\infty \\
\infty \\
\stackrel{+}{+}\end{array}$ & 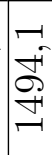 & $\begin{array}{l}1 \\
2 \\
0 \\
20 \\
10 \\
-1\end{array}$ & $\mid \begin{array}{c}0 \\
0 \\
20 \\
00 \\
\qquad \\
= \\
\end{array}$ & 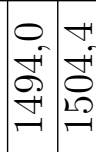 & 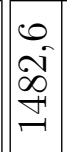 & 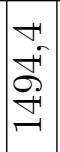 & 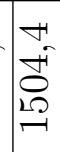 \\
\hline & จ & $\mid \begin{array}{l}20 \\
\sim \\
\sim\end{array}$ & $\stackrel{\vec{N}}{ }$ & $\stackrel{\mathscr{L}}{\mathrm{N}}$ & $\stackrel{\infty}{\sim}$ & $\stackrel{\sim}{\curvearrowright}$ & $\stackrel{\vec{N}}{\sim}$ & $\stackrel{\infty}{\sim}$ & $\stackrel{\sim}{\curvearrowright}$ & $\stackrel{\sim}{\sim} \underset{\sim}{\stackrel{\infty}{N}}$ & 尺ิ & $\vec{\sim}$ & $\stackrel{\infty}{\sim}$ \\
\hline ن & & & 0 & & & & 10 & & & L2 & & $=$ & \\
\hline
\end{tabular}




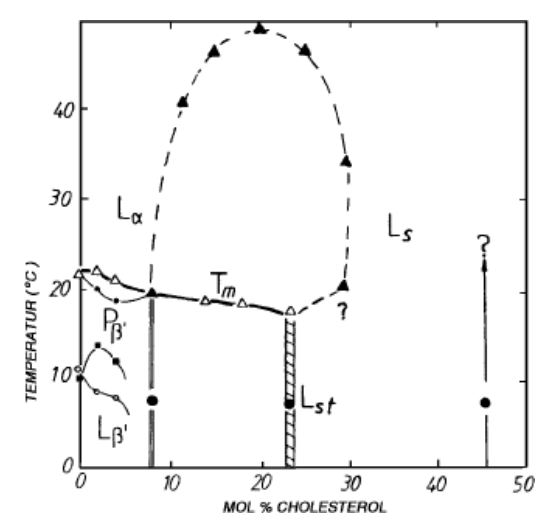

Abbildung 6.7: Phasendiagramm von DMPC/Cholesterol-Mischungen. Oberhalb eines Cholesterolgehaltes von $7,5 \mathrm{~mol} \%$ setzt eine Separation in cholesterolarme und cholesterolreiche Phasen ein [78].

Das Phasendiagramm von DMPC/Cholesterol-Mischungen in Abb. 6.7 [78] zeigt eine komplizierte Abhängigkeit der Phasenübergänge vom Cholesterolgehalt. Bei geringen Konzentrationen mit $c_{\text {chol }}<7,5 \mathrm{~mol} \%$ bewirkt Cholesterol lediglich eine leichte Verringerung der Hauptumwandlungstemperatur zwischen der gelförmigen Phase $\left(L_{\beta^{\prime}}, P_{\beta^{\prime}}\right)$ und der fluiden Phase $\left(L_{\alpha^{\prime}}\right)$. Für $c_{\text {chol }}>7,5 \mathrm{~mol} \%$ findet eine Separation in eine cholesterolreiche Phase $L_{\mathrm{s}}$ (flüssig-geordnete Phase) und eine cholesterolarme Phase $\left(L_{\beta^{\prime}}, L_{\alpha^{\prime}}\right)$ statt. Die separierten Phasen können mit der Ultraschallspektrometrie nicht innerhalb der bekannten Modelle beschrieben werden. Deswegen soll die Diskussion auf $c_{\text {chol }}<7,5 \mathrm{~mol} \%$ beschränkt werden.

Mouritsen und Jørgensen [87] führen neben der Reduzierung der Übergangstemperatur auch einen Einfluss auf die Domänenbildung an. Molekulardynamik-Simulationen belegen, dass Cholesterol das Entstehen von Domänen begünstigt, während es sich selber in der Nähe der Domänengrenzen ansammelt.

In Messungen von Michels et al. [84] mittels Ultraschallspektrometrie konnte ein Anstieg der kritischen Fluktuationen nachgewiesen werden. Die in Tab. 6.8 zusammengestellten Daten zeigen diesen Anstieg der kritischen Amplitude $S$ mit wachsender Cholesterolkonzentration. Gleichzeitig führt die Anlagerung in der Nähe der Domänengrenzen zu ihrer Stabilisierung. Daraus resultierend ist eine Verringerung der kritischen Relaxationsrate $\Gamma$ zu erwarten, die ebenfalls in Tab. 6.8 dokumentiert ist.

Die Zugabe von Alamethicin bewirkt eine deutliche Reduktion der Relaxationsrate $\Gamma$. Ein vergleichbarer Effekt kann, wie soeben beschrieben, bei Zugabe von Cholesterol zu einer DMPC-Membran beobachtet werden. Hier tritt eine konzentrationsabhängige Reduktion der Relaxationsrate in gelförmiger und fluider Phase von 25\% für Cholesterolkonzentrationen $c_{\text {chol }} \leq 7,5 \mathrm{~mol} \%$ auf. Bei Zugabe von $10 \mathrm{~mol} \%$ Cholesterol wird die Relaxationsrate sogar um $50 \%$ reduziert, jedoch ist bei dieser Konzentration von einer Phasenseparation auszugehen.

Alamethicin zeigt die gleiche Reduktion bereits bei einer Konzentration von 0,3 mol\% bis $1,0 \mathrm{~mol} \%$.

Dies deutet auf den Einfluss der Molekülgröße hin. Cholesterol bettet sich in den hydrophoben Teil der Moleküle ein und stabilisiert diese in der all-trans-Konfiguration. Der 
Einfluss eines Cholesterolmoleküls ist auf eine Seite der Membran beschränkt.

Die Helix des Alamethicin wird in beide Seiten der Membran eingebettet und führt dadurch zu einer Beeinflussung beider Membranlipidschichten. Gleichzeitig besitzt Alamethicin polare Enden, die gerichtete Bindungen mit den polaren Kopfgruppen der Lipide aufbauen. Die Länge des Alamethicinmoleküls entspricht der Dicke einer DMPC-Membran in der fluiden Phase. Um die Grenzflächenenergie der Membran in der Nähe des Peptids zu minimieren, werden die umgebenden DMPC-Moleküle in der fluiden Phase stabilisiert.

Diese Stabilisierung und die Verankerung des Peptids in beiden Membranseiten führt zu einer Reduktion der Relaxationsrate schon bei geringen Alamethicinkonzentrationen.

Bei Konzentrationen $c_{\text {alm }} \leq 0,5 \mathrm{~mol} \%$ wird das Peptid auf der Membranoberfläche adsorbiert. Die soeben beschriebene Theorie der Stabilisierung kann nicht auf adsorbierte Moleküle angewendet werden. Es ist auch hier eine Stabilisierung der kritischen Fluktuationen feststellbar.

Die Einlagerung des Peptids in den Kopfgruppenbereich der Lipidmembran führt zu einer lokalen Deformation, die mit einem Aufschmelzen der Membran einhergeht. Die Membran wird hier also wieder in der fluiden Phase stabilisiert, wodurch die Fluktuationsrate verringert wird. Weiterhin ist ein möglicher stabilisierender Faktor durch das Hydratwasser der Membran denkbar. Die Beweglichkeit der Wassermoleküle ist aufgrund der Bindung zum Peptid reduziert, folglich ist auch eine Stabilisierung der Lipid-Wasser-Bindungen zu erwarten.

Die Frage, ob sich Alamethicin ähnlich wie Cholesterol an den Domänengrenzen sammelt, kann mit Hilfe der zur Verfügung stehenden Methoden nicht geklärt werden. Jedoch ist bekannt, dass Alamethicin in der gelförmigen Phase statistisch in der Membran verteilt ist $[57,112]$. Demzufolge müsste eine Migration des Peptids in der Nähe des Phasenübergangs stattfinden.

\subsubsection{Die axiale Diffusion}

Die axiale Diffusion eines einzelnen Lipids wird bestimmt durch die Beweglichkeit wie in Abb. 6.1 veranschaulicht wurde. Die Adsorption des Peptids bei niedrigen Konzentrationen und Einbettung in die Kopfgruppenregion führt zu lokalen Deformationen und zu einer Erhöhung der Membranspannung. Die in Abb. 6.1 dargestellte laterale Verschiebung erfordert nun eine höhere Energie, läuft daher nach der Arrheniusgleichung [86] langsamer $\mathrm{ab}$ :

$$
k \sim e^{-E_{\mathrm{a}} /(R T)} \Rightarrow \tau \sim e^{E_{\mathrm{a}} /(R T)}
$$

mit

$$
\begin{aligned}
& k: \text { Reaktionsrate } \\
& \tau: \text { Relaxationszeit }
\end{aligned}
$$

$\Delta H_{0}$ : Aktivierungsenergie der Reaktion

$R, T$ : universelle Gaskonstante und Temperatur. 

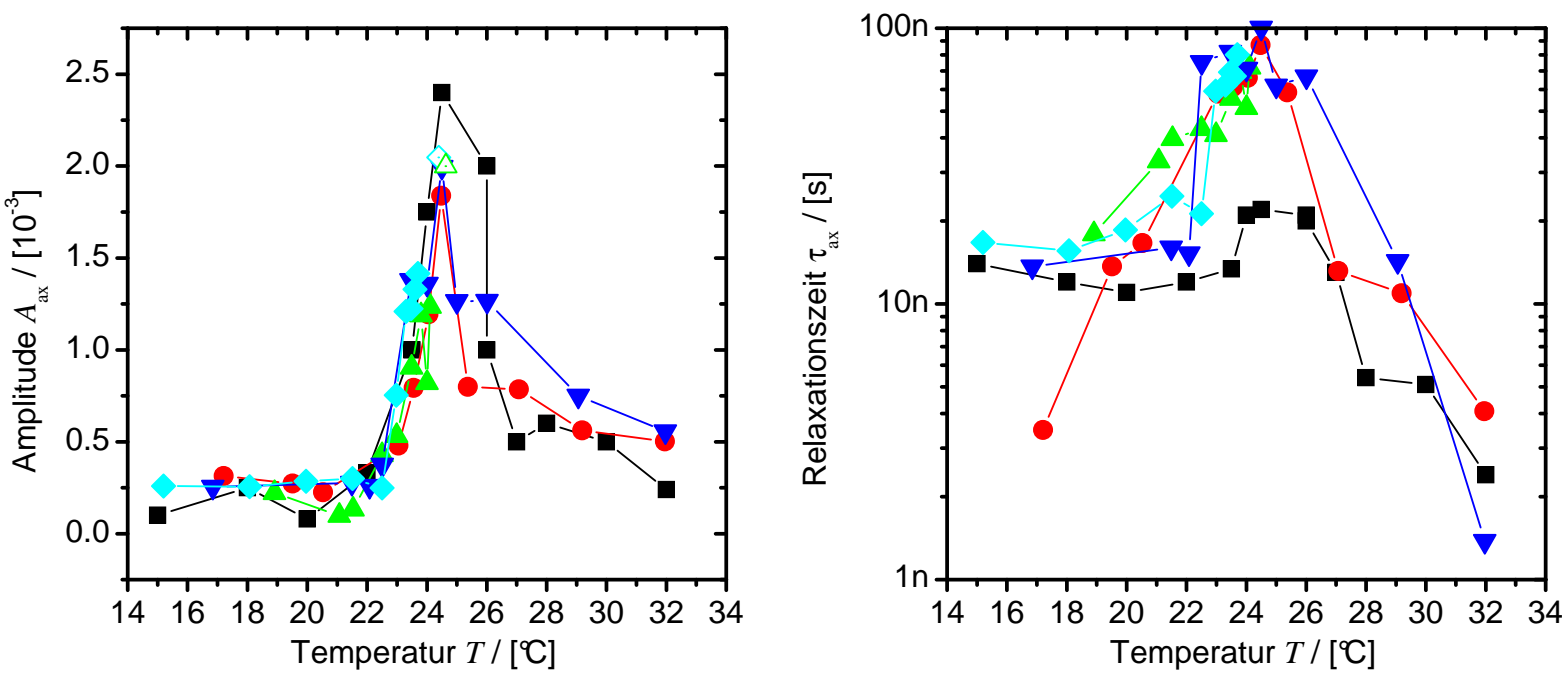

Abbildung 6.8: Relaxationszeiten und Amplituden der axialen Diffusion. In dem linken Graph ist die Amplitude der axialen Diffusion dargestellt; im rechten Graph ist die Relaxationszeit

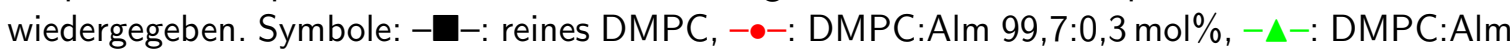
99,5:0,5 mol\%, - - -: DMPC:Alm 99,3:0,7 mol\%, - -: DMPC:Alm 99:1 mol\%.

Nach Tieleman [108] geht Alamethicin stabilisierende Bindungen mit den polaren Kopfgruppen der Lipide ein, die zu einer zusätzlichen Immobilisierung der Lipide führen.

Die Immobilisierung der Moleküle durch die Adsorption und die höheren Energien, die zur Rotation notwendig sind, sollten zu einer Reduktion der Amplitude und einer Verlangsamung der axialen Diffusion führen, die anhand der Messdaten für Membranen mit $0,3 \mathrm{~mol} \%$ und $0,5 \mathrm{~mol} \%$ Alamethicin eindeutig belegt ist.

Die Integration der Alamethicinmoleküle bei höheren Konzentrationen bewirkt ebenfalls eine Störung der Kopfgruppenregionen. In der Nähe des eingebetteten Peptids wird mittels Rasterkraftmikroskopie [89] eine Reduzierung der Membrandicke festgestellt, der als Rand um die Poren in Abb. 6.9 zu sehen ist.
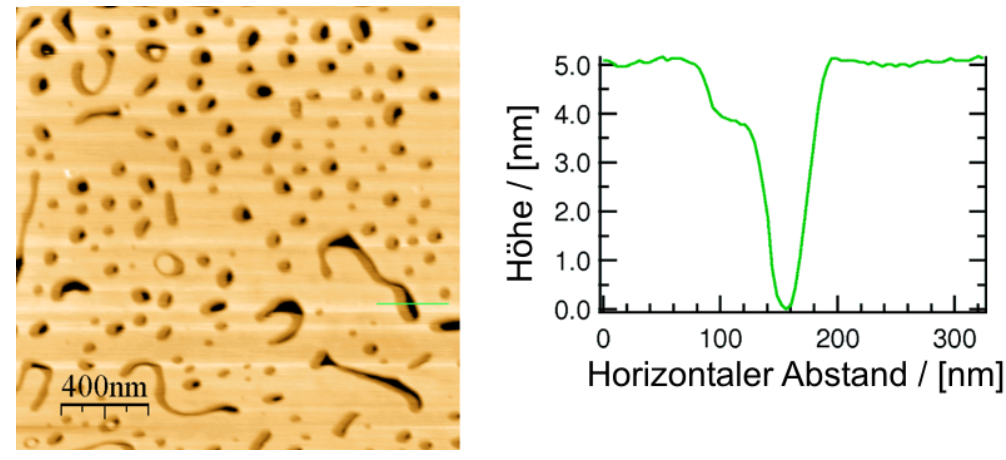

\begin{abstract}
Abbildung 6.9: Rasterkraftmikroskopische Aufnahme einer alamethicinhaltigen Membran. Das linke Bild zeigt eine Aufnahme mit dem Rasterkraftmikroskop einer alamethicinhaltigen DPPC-Membran (DPPC:Alm, 99:1 mol\%). Auf dem linken Rand der dunklen Poren sind Ränder zu erkennen. Entlang der grünen Linie wurde das auf der rechten Seite dargestellte Höhenprofil erstellt [89].
\end{abstract}

Die Porenbildung und die Ausbildung eines deutlichen Randes scheinen keinen Einfluss 

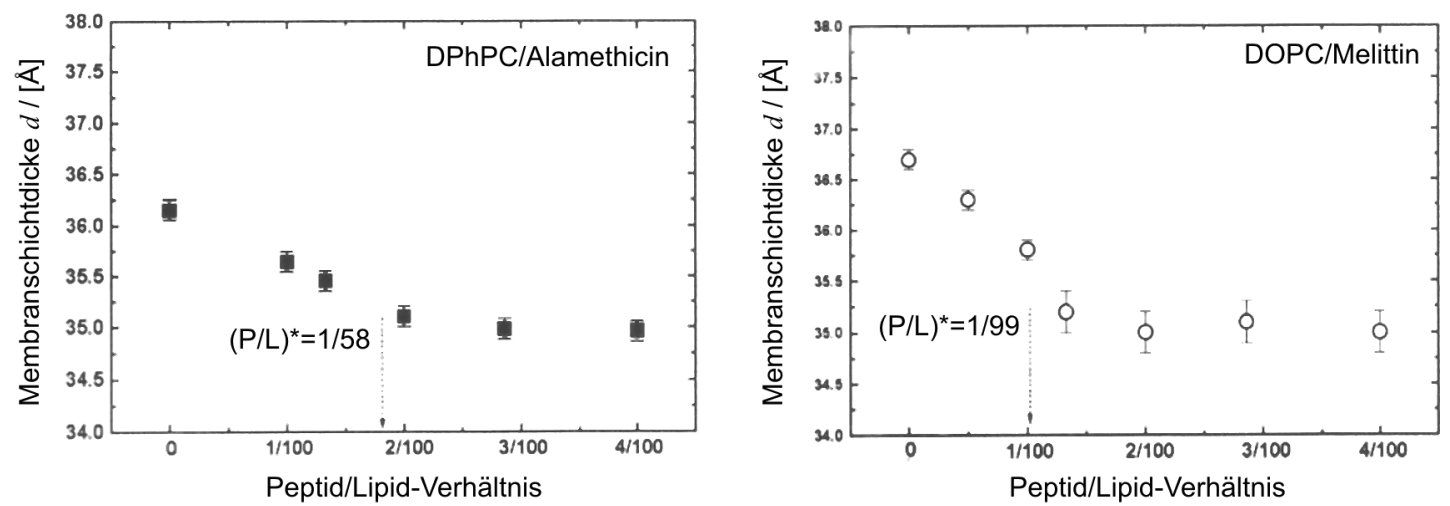

Abbildung 6.10: Reduzierung der Membrandicke von alamethicin- und melittinhaltigen Membranen. Die Graphen zeigen die Änderung der Membrandicke in DPhPC und DOPC bei Zugabe der $\alpha$-helikalen Peptide Alamethicin und Melittin, das in seiner Wirkung vergleichbar mit Alamethicin ist. $(P / L)^{*}$ ist die kritische Integrationskonzentration [29].

auf die Amplitude und die Relaxationszeit der axialen Rotation zu haben. Sie entspricht innerhalb der Messgenauigkeit den Werten für die Adsorption auf der Membranoberfläche. Nach [29, 46] bleiben die Membrandicke und damit auch die Erhöhung der Membranspannung während der Integration des Peptids konstant, wie in Abb. 6.10 dargestellt ist.

Der Vergleich der Membrandicke mit der Relaxationsrate und der Amplitude der axialen Diffusion weist eine Beeinträchtigung der axiale Diffusion durch die Membrandeformation auf. In Abb. 6.11 sind zum Vergleich noch einmal die Parameter der axialen Diffusion in der Nähe der kritischen Temperatur für verschiedene Alamethicinkonzentrationen dargestellt.

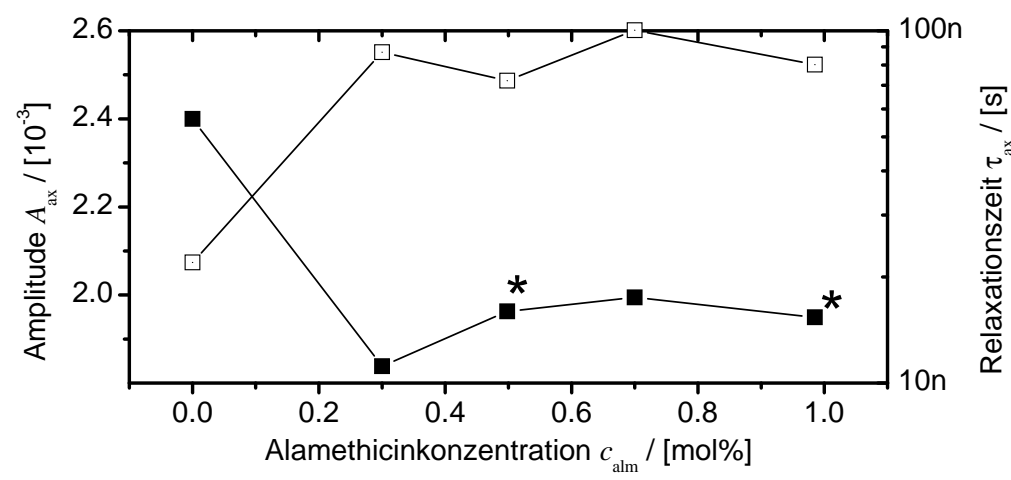

Abbildung 6.11: Parameter der axialen Diffusion in der Nähe der kritischen Temperatur. Die Abbildung zeigt den Verlauf der Relaxationszeit $\tau_{\text {ax }}(-\square-)$ und der Amplitude $A_{\text {ax }}(-\mathbf{-}-)$ der axialen Diffusion. Die mit $(\star)$ markierten Werte wurden anhand des Amplitudenverlaufs interbzw. extrapoliert.

Wie von Schrader et al. [100] angenommen wird, ist die axiale Diffusion $D_{\text {rot }}$ an die laterale Diffusion $D$ gekoppelt. In den Messdaten ist ein deutlicher Anstieg der Relaxationszeit in der Nähe der kritischen Temperatur des alamethicinhaltigen Systems gegenüber dem reinen DMPC-System zu erkennen. Dieses lässt auf eine stärkere Kopplung der axialen Diffusion an die laterale Diffusion schließen. 


\subsubsection{Die Vesikeldeformation}
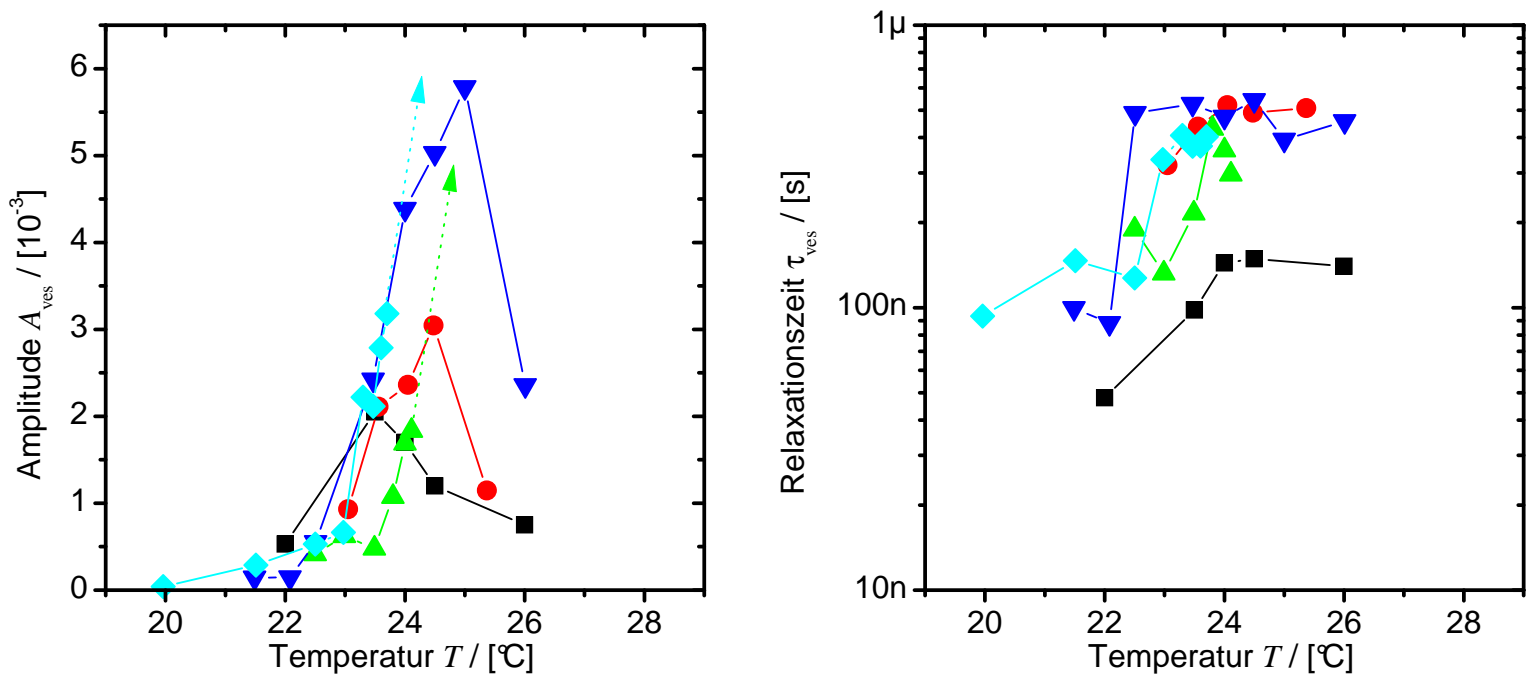

Abbildung 6.12: Relaxationszeiten und Amplituden der Vesikeldeformation. In dem linken Graph ist die Amplitude der Relaxation der Vesikeldeformation dargestellt; im rechten Graph ist die zugehörige Relaxationszeit wiedergegeben. Symbole: - - -: reines DMPC, - $\bullet$-:

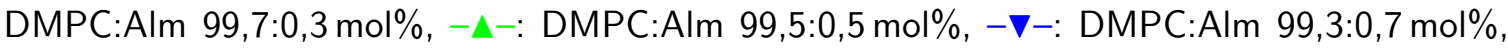
$-\rightarrow$ DMPC:Alm 99:1 mol\%.

Bei der Interpretation der Spektren ist die Einführung einer dritten Einzelzeitrelaxation mit einer Relaxationszeit von $\tau \leq 100$ ns erforderlich. Dieser Term wird von Schrader et al. mit einer lokalen Vesikeldeformation in Verbindung gebracht [100].

Die lokale Vesikeldeformation wurde mittels optischer Methoden an $15 \mu \mathrm{m}$ großen unilamellaren Vesikeln aus 1,2-Dipalmitoyl-sn-Glycerin-3-Phosphatidylcholin (DPPC) untersucht [76]. Dazu wurde mittels eines Nd:YAG-Lasers (532nm) eine Kraft von $53 \mathrm{fN}$ auf das Vesikel ausgeübt. Der Radius des Vesikels ändert sich auf einer Zeitskala von wenigen Sekunden (Abb.6.13) um $600 \mathrm{~nm}$ entlang der optischen Achse.

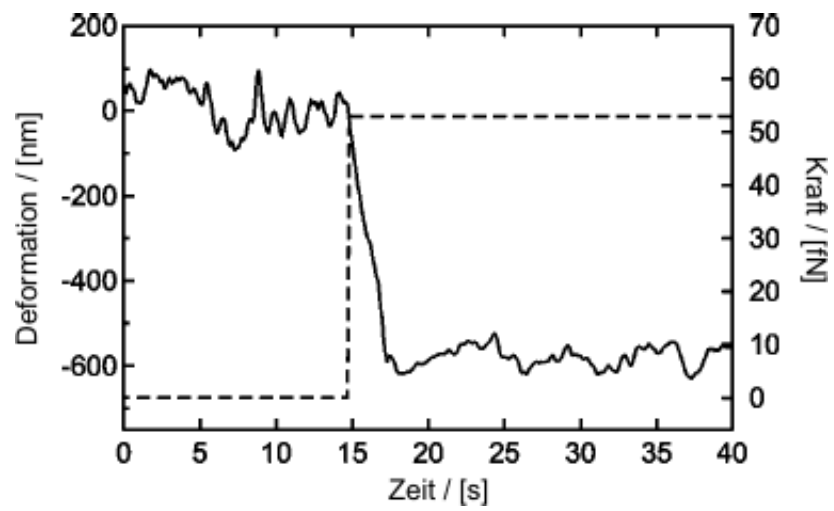

Abbildung 6.13: Deformation eines DPPC-Vesikels. Bei Anlegen einer Kraft von $53 \mathrm{fN}$ durch einen Nd:YAG-Laser wird eine lokale Deformation von $600 \mathrm{~nm}$ bei einer Vesikelgröße von $15 \mu \mathrm{m}$ erzeugt. Die durchgezogene Linie zeigt die Änderung des Vesikelradius entlang der optischen Achse, die gestrichelte die angelegte Kraft [76]. 
Das Experiment wurde bei $30^{\circ} \mathrm{C}$ durchgeführt, weit unterhalb der Übergangstemperatur von DPPC. Während die Membran fern der Übergangstemperatur homogen ist, so ist sie am Übergang in zahlreiche Domänen gegliedert, deren Größe und Gestalt sich kontinuierlich ändert.

Schrader et al. nehmen an, dass daher in der Nähe des Phasenübergangs Fluktuationen auf einer kurzen Längenskala mit Relaxationszeiten im Zeitbereich unter einer Millisekunde existieren [100]. Diese Annahme muss immer noch bestätigt werden, aber die Messungen an alamethicinhaltigen DMPC-Membranen liefern einen weiteren Hinweis für die Richtigkeit dieser Annahme.

Alamethicin als antimikrobiales Peptid besitzt eine lysierende Wirkung auf die Zellmembran. Diese Wirkung wird von Huang et al. [57] der Porenbildung zugeschrieben. Mit der Entstehung einer Pore ist die Vergrößerung der inneren Membranspannung $\sigma_{\text {int }}$ verbunden. Diese Theorie wird durch Versuche gestützt, in denen eine externe mechanische Spannung $\sigma_{\text {ext }}$ appliziert wird, die zur Lysis der Membran führt $[59,79,101]$. Dabei stellt sich die „Lysierungsspannung“ $\sigma_{\text {lys }}$ als Summe von interner und externer Membranspannung dar:

$$
\sigma_{\mathrm{lys}}=\sigma_{\mathrm{int}}+\sigma_{\mathrm{ext}} .
$$

Die Membran bleibt stabil, so lange $\sigma_{\text {lys }}>\sigma_{\text {int }}+\sigma_{\text {ext }}$, d. h. für die Zerstörung der Membran sind mit steigendem Peptidgehalt geringere äußere Kräfte notwendig.

Alle bisher im Rahmen dieser Arbeit diskutierten Ergebnisse von peptidhaltigen DMPCMembranen beziehen sich auf Alamethicinkonzentrationen von $c_{\mathrm{alm}} \leq 1 \mathrm{~mol} \%$.

Messungen an alamethicinhaltigen DMPC-Membranen mit $c_{\text {alm }}=2 \mathrm{~mol} \%$ scheiterten, weil die Lösung in der Nähe der Umwandlungstemperatur bei allen Versuchen präzipitiert ist. Die Präzipitation setzt eine Lysis der bestehenden Vesikel und eine Reorganisation der Membranfragmente voraus.

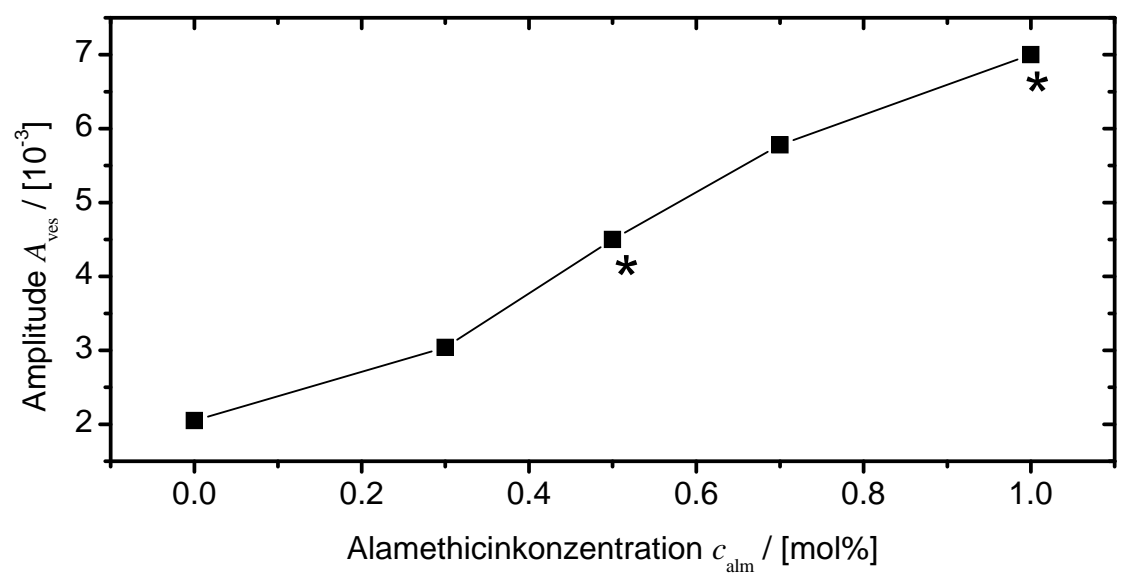

Abbildung 6.14: Amplitude der Vesikeldeformation in der Nähe der kritischen Temperatur. Die Abbildung zeigt den Verlauf der Amplitude $A_{\text {ves }}(-\boldsymbol{-}-)$ der Vesikeldeformation in der Nähe der kritischen Temperatur. Die mit $(*)$ markierten Werte wurden inter- bzw. extrapoliert.

Eine Erklärung für dieses Verhalten wird durch die Vesikeldeformation geliefert. In Abb. 6.12 erkennt man einen starken Anstieg der Amplitude der Vesikeldeformation mit 
steigendem Alamethicingehalt. Die Amplitudenmaxima nahe der kritischen Temperatur sind in Abb. 6.14 zur Verdeutlichung noch einmal separat dargestellt. Es ist ein Anstieg der Amplitude bei $c_{\text {alm }}=1$ mol\% auf das Dreifache im Vergleich zu reinem DMPC zu erkennen. Unter der Annahme, dass sich nach Gl. (3.16) das Reaktionsvolumen $\Delta V$, die Reaktionsenthalpie $\Delta H$ und die Wärmekapazität $\Delta C_{\mathrm{p}}$ bei Zugabe Alamethicin nur geringfügig ändern, ist der Anstieg der Amplitude auf die Änderungen der Stöchiometriefaktoren $\nu_{i}$ in

$$
G=\frac{\sum \nu_{i}^{2}}{c_{A_{i}}}
$$

zurückzuführen. Die Zahl der deformierten Bereiche nimmt also stark zu.

Das zuvor beschriebene Experiment von Lee [76] zeigt, dass eine äußere angelegte mechanische Spannung zu einer Vesikeldeformation führt. Umgekehrt muss die Vesikeldeformation ebenfalls zu einer zusätzlichen Membranspannung führen. Gl. (6.7) ist demnach in der Nähe der kritischen Temperatur zu modifizieren:

$$
\sigma_{\mathrm{lys}}=\sigma_{\mathrm{int}}+\sigma_{\mathrm{ext}}+\sigma_{\mathrm{ves}},
$$

wobei $\sigma_{\text {ves }}$ die durch die Vesikeldeformation erzeugte Membranspannung berücksichtigt.

Bei einer Konzentration von $c_{\text {alm }}=2 \mathrm{~mol} \%$ wird $\sigma_{\text {lys }}$ überschritten und die Lysis setzt ein.

Die Relaxationszeiten der Vesikeldeformation bei alamethicinhaltigen Membranen liegen deutlich oberhalb derer der reinen DMPC-Membran. Dieses erlaubt zwei Schlussfolgerungen:

1. Die Kopplung an das kritische Verhalten und dem damit verbundenen Critical Slowing Down wird durch die Anwesenheit des Alamethicin verstärkt.

2. Das Alamethicin führt aufgrund seiner Verankerung in beiden Membranhälften zu einer Stabilisierung der Membran und deren Deformationen.

Eine Beschreibung, in welchem Maße die einzelnen Effekte die Relaxationszeiten beeinflussen, kann in dieser Arbeit nicht gegeben werden. 


\section{Messergebnisse aus Densitometrie und Velocimetrie}

\subsection{Temperaturabhängige Untersuchungen von Dichte und Schallgeschwindigkeit}

Die Membrane Thinning-Theorie [29, 46], die das Volumenverhalten der Membran während der Adsorption und Integration von $\alpha$-helikalen Peptiden beschreibt, liefert, wie das letzte Kapitel gezeigt hat, bedeutende Beiträge zur Erklärung der Ultraschallspektren. Jedoch wurde diese Theorie, die den Volumenerhalt der Membran bei Adsorption und Integration vorhersagt, in den vorhandenen Veröffentlichungen nur in der gelförmigen Phase, weit unterhalb der Übergangstemperatur des jeweiligen Lipids überprüft.

Mit Hilfe der temperaturabhängigen Densitometrie kann der Aspekt der Volumenerhaltung am Phasenübergang für verschiedene Alamethicinkonzentrationen leicht verifiziert werden.

Dazu wurde die Dichte $\rho(T)$ der DMPC/Alamethicin-Gemische relativ zu der Dichte von Wasser $\rho_{\mathrm{H}_{2} \mathrm{O}}(T)$ aufgetragen, um die Dichteänderung des Phasenübergangs von DMPC herauszustellen:

$$
\rho_{\text {rel }}=\frac{\rho(T)}{\rho_{\mathrm{H}_{2} \mathrm{O}}(T)} .
$$

Um präparationsbedingte Konzentrationsunterschiede zu kompensieren, die linear in die relative Dichte eingehen, wurden die Daten auf $\rho_{\text {rel }}\left(T=15^{\circ} \mathrm{C}\right)=1$ normiert. Die normierte relative Dichte ist in Abb. 7.1 wiedergegeben.

Entsprechend der Membrane Thinning-Theorie ändert sich das temperaturabhängige Dichteverhalten und damit das Volumenverhalten bei Zugabe von Alamethicin nicht. Für DMPC:Alamethicin, 98:2 mol\% konnte nach Ende der Messung eine Ausfällung des Lipids beobachtet werden (Symbol $(+)$ ). Die Präzipitation erklärt die graduelle Verringerung der relativen Dichte.

Die übrigen Kurven zeigen ein nahezu identisches Verhalten. Vorhandene Abweichungen sind als Messfehler zu interpretieren, da keine Systematik in Zusammenhang mit der Alamethicinkonzentration zu erkennen ist.

Mittels des in Abschnitt 4.4 vorgestellten Velocimetrie-Aufbaus wurde zusätzlich die Schallgeschwindigkeit der Proben gemessen. Wie Abb. 7.3 (a) zeigt, kann auch hier keine Abhängigkeit von der Alamethicinkonzentration festgestellt werden. 


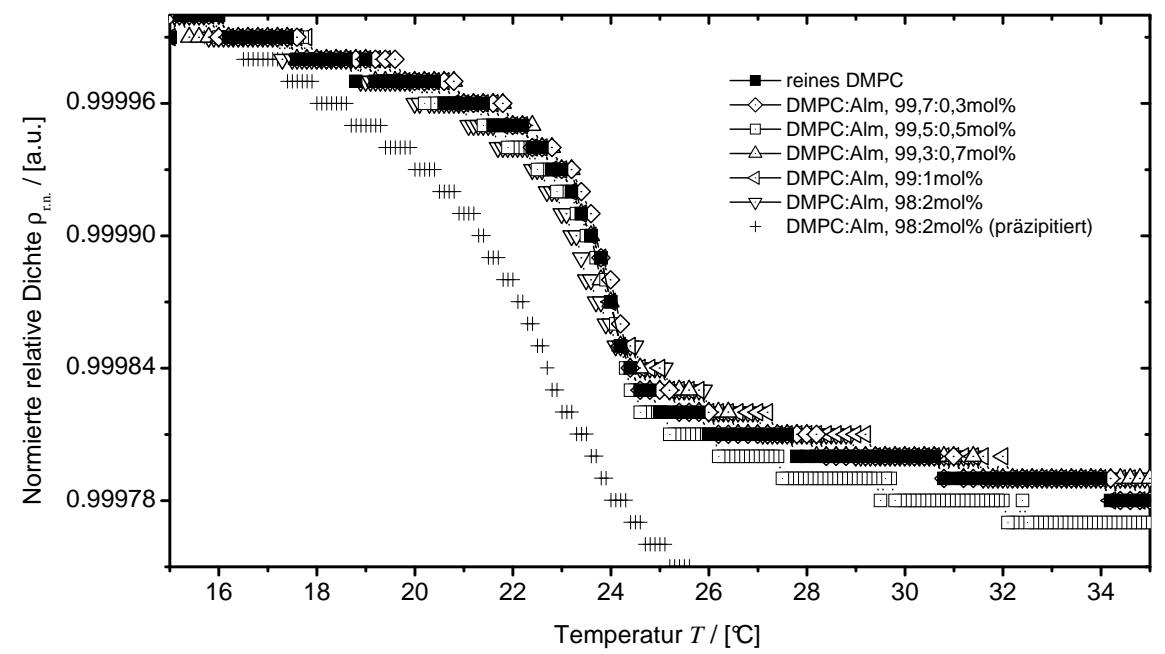

Abbildung 7.1: Darstellung der normierten relative Dichte. In dieser Grafik ist die normierte relative Dichte gegen die Temperatur in der Umgebung des Phasenübergangs aufgetragen.

Ziel der Messungen von Schallgeschwindigkeit und Dichte ist die Bestimmung der Kompressibilität der Membran. Nach Gl. (4.30) und Gl. (4.31) sind die relativen Größenänderungen der Dichte $[\rho]$ und der Schallgeschwindigkeit $\left[c_{\mathrm{s}}\right]$ direkt zugängliche Größen der Lösung. In der physikalischen Chemie werden aber häufig das so genannte scheinbare molare Volumen $\phi_{\mathrm{V}}$ und die scheinbare molare Kompressibilität $\phi_{\mathrm{KS}}$ zur Beschreibung des Volumens und der elastischen Eigenschaften verwendet. Sie werden definiert durch [97]

$$
\begin{aligned}
\phi_{\mathrm{V}} & =\frac{V-V_{0}}{V \cdot k}=\frac{M}{\rho_{0}}-[\rho] \\
\phi_{\mathrm{KS}} & =\frac{\kappa_{\mathrm{S}}-\kappa_{\mathrm{s}, 0}}{V \cdot k},
\end{aligned}
$$

mit

$V, \rho, \kappa_{\mathrm{S}}$ : Volumen, Dichte und adiabatische Kompressibilität der Lösung $V_{0}, \rho_{0}, \kappa_{\mathrm{s}, 0}$ : wie zuvor, für auf das Lösungsmittel

$M, k$ : Molmasse und Konzentration der gelösten Substanz.

Mit Gl. (4.32) und Gl. (4.34) folgt für die scheinbare molare Kompressibilität aus den Messgrößen

$$
\phi_{\mathrm{KS}}=2 \kappa_{\mathrm{s}, 0}\left(\phi_{\mathrm{V}}-\left[c_{\mathrm{s}}\right]-\frac{M}{2 \rho}\right)
$$

Die adiabatische Kompressibilität des Lösungsmittels $\kappa_{\mathrm{s}, 0}$, in diesem Fall des Wassers, lässt sich nach Gl. (3.6) aus der Dichte und der Schallgeschwindigkeit des Lösungsmittels, die als bekannte Größen vorausgesetzt werden, bestimmen.

Abb. 7.2 zeigt das scheinbare molare Volumen, bezogen auf ein Mol DMPC. Da Alamethicin in der Membran nur in sehr geringen Konzentrationen vorhanden ist, kann Alamethicin in dieser Betrachtung vernachlässigt werden. 


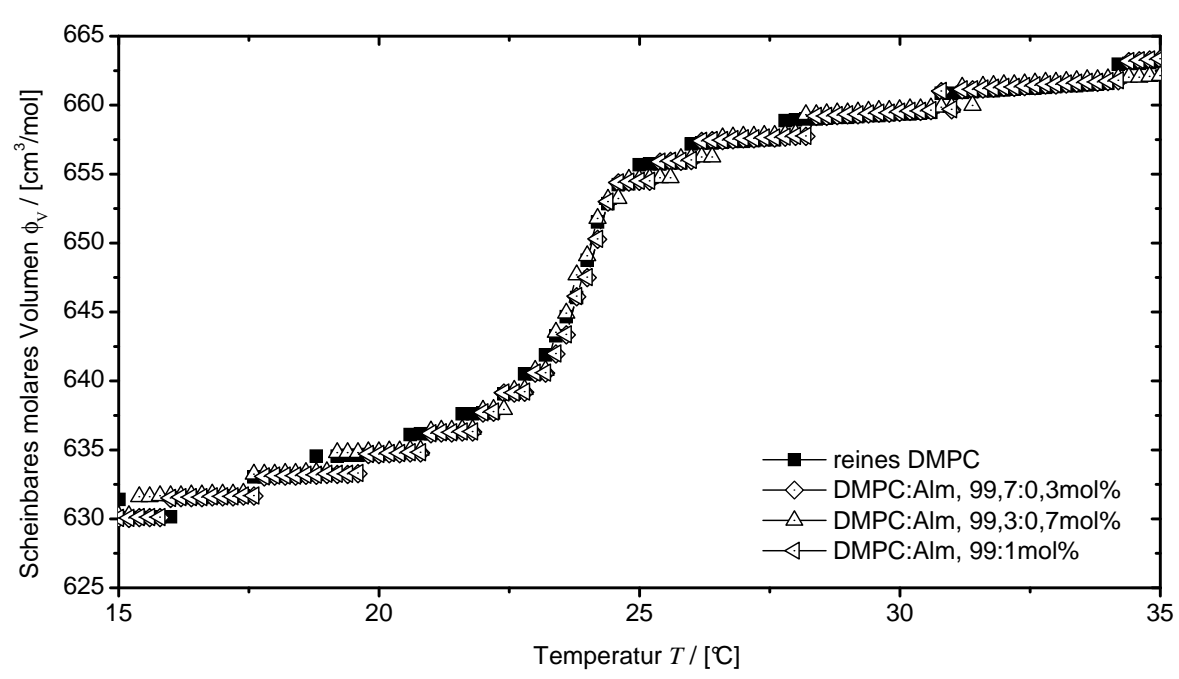

Abbildung 7.2: Scheinbares molares Volumen. Die Abbildung zeigt das scheinbare molare Volumen der Membran am Phasenübergang bei verschiedenen Alamethicinkonzentrationen.

Im Falle des DMPC ändert sich das intrinsische Volumen während des Phasenübergangs durch das Schmelzen der Alkylketten. Hydratationseffekte spielen eine untergeordnete Rolle, da das Molekül nur über die hydrophilen Kopfgruppen Bindungen zum Wasser ausbilden kann, während sich die Alkylketten, die eine Konformationsänderung durchlaufen im hydrophoben Innenraum der Membran befinden.

In Abb. 7.3 ist die auf die Konzentration normierte Schallgeschwindigkeitszahl $\left[c_{\mathrm{s}}\right]$ dargestellt. Ebenso wie bei den densitometrischen Untersuchungen zeigen sich hier keine Veränderungen bei steigendem Alamethicingehalt.

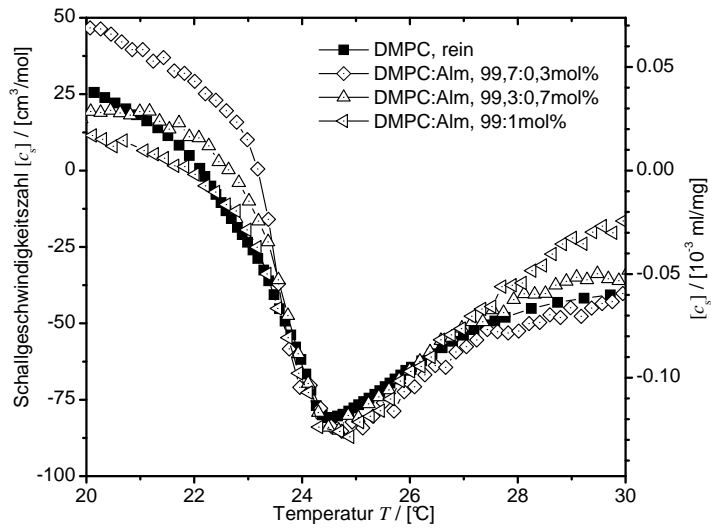

(a) Alamethicin

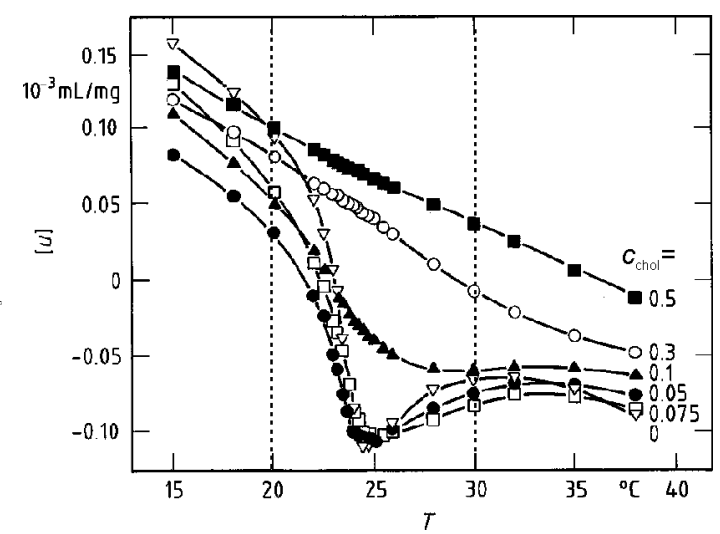

(b) Cholesterol

Abbildung 7.3: Auftragung der Schallgeschwindigkeitszahl für Alamethicin und Cholesterol. In der linken Abbildung ist die Schallgeschwindigkeitszahl $\left[c_{\mathrm{s}}\right]$ aufgetragen in $\left[\mathrm{cm}^{3} / \mathrm{mol}\right]$ und $\left[\mathrm{cm}^{3} / \mathrm{mg}\right]$. In der rechten Abbildung aus [41] ist $\left[c_{\mathrm{s}}\right]=[u]$ in $\left[\mathrm{cm}^{3} / \mathrm{mg}\right]$ aufgetragen.

Die Messung der Schallgeschwindigkeitszahl ist äquivalent zur differenziellen Kalorimetrie wie von Heimburg [49] durch das Dissipations-Fluktuationsgesetz für Membransysteme 
gezeigt wurde. Dabei gilt nach Gl. (2.6) für die isotherme Kompressibilität

$$
\kappa_{\mathrm{T}}=\frac{\gamma^{2} T}{\langle V\rangle} \Delta C_{\mathrm{p}}
$$

welche über die Maxwell-Relationen mit der adiabatischen Kompressibilität $\kappa_{\mathrm{S}}$ verknüpft ist. Mit dem Anstieg von $C_{\mathrm{p}}$ nach dem Dissipations-Fluktuationsgesetz am Phasenübergang und der Beziehung $c_{\mathrm{S}}=\sqrt{1 /\left(\kappa_{\mathrm{S}} \rho\right)}$ nimmt die Schallgeschwindigkeit am Phasenübergang ein Minimum an. Aus den Messdaten, dargestellt in Abb.7.3 (a) ist unter Berücksichtigung des Messfehlers von 10\% keine eindeutige Änderung der Übergangstemperatur in Abhängigkeit vom Alamethicingehalt zu erkennen.

Velocimetriedaten von Cholesterolsystemen [41] zeigen für Cholesterolkonzentrationen $c_{\text {chol }} \leq 7,5 \mathrm{~mol} \%$ ebenfalls keine Änderung der Übergangstemperatur, wie man aus Abb. 7.3 entnehmen kann.

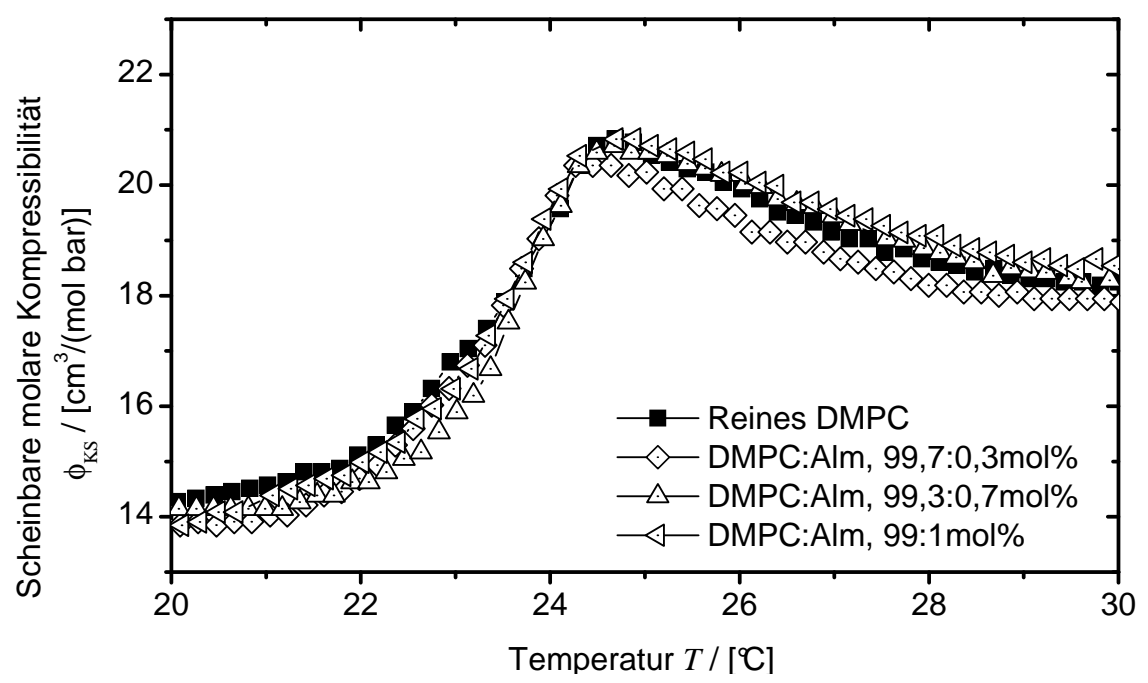

Abbildung 7.4: Darstellung der scheinbaren molaren Kompressibilität. Die Grafik zeigt die scheinbare molare Kompressibilität am Phasenübergang bei verschiedenen AlamethicinKonzentrationen.

Aus den Daten der Densitometrie und Velocimetrie wurde nach Gl. (7.4) die scheinbare molare Kompressibilität berechnet, die in Abb. 7.4 dargestellt ist.

Die Änderung der Kompressibilität kann auf die Volumenvergrößerung während des Schmelzens zurückgeführt werden. In unmittelbarer Nähe des Phasenübergangs stellt man ein Maximum der Kompressibilität fest. Dieses ist auf den Anstieg der kritischen Fluktuationen an der kritischen Temperatur zurückzuführen.

Der Vergleich mit cholesterolhaltigen Systemen zeigt, dass sowohl alamethicin- als auch cholesterolhaltige Systeme nur eine marginale Verschiebung der Umwandlungstemperatur aufweisen. Dieses kann als Indiz gewertet werden, dass Alamethicin und Cholesterol auf ähnliche Weise das Membranverhalten modifizieren. 


\subsection{Untersuchung der Schallgeschwindigkeit in Abhängigkeit von der Alamethicinkonzentration}

Neben der temperaturabhängigen Untersuchung der Schallgeschwindigkeit wurde auch die Änderung der Schallgeschwindigkeit in Abhängigkeit von der Alamethicinkonzentration untersucht. Dazu wurden wie in Abschnitt 4.4.2 eine Referenztitration mit reinem Ethanol und eine Substanztitration mit einer Ethanol/Alamethicin-Lösung der Konzentration $k_{\text {alm }}=5,102 \mu \mathrm{mol} / \mathrm{ml}$ durchgeführt. Die Lösung der DMPC-Suspension betrug $k_{\mathrm{DMPC}}=$ $7,538 \mu \mathrm{mol} / \mathrm{ml}$.

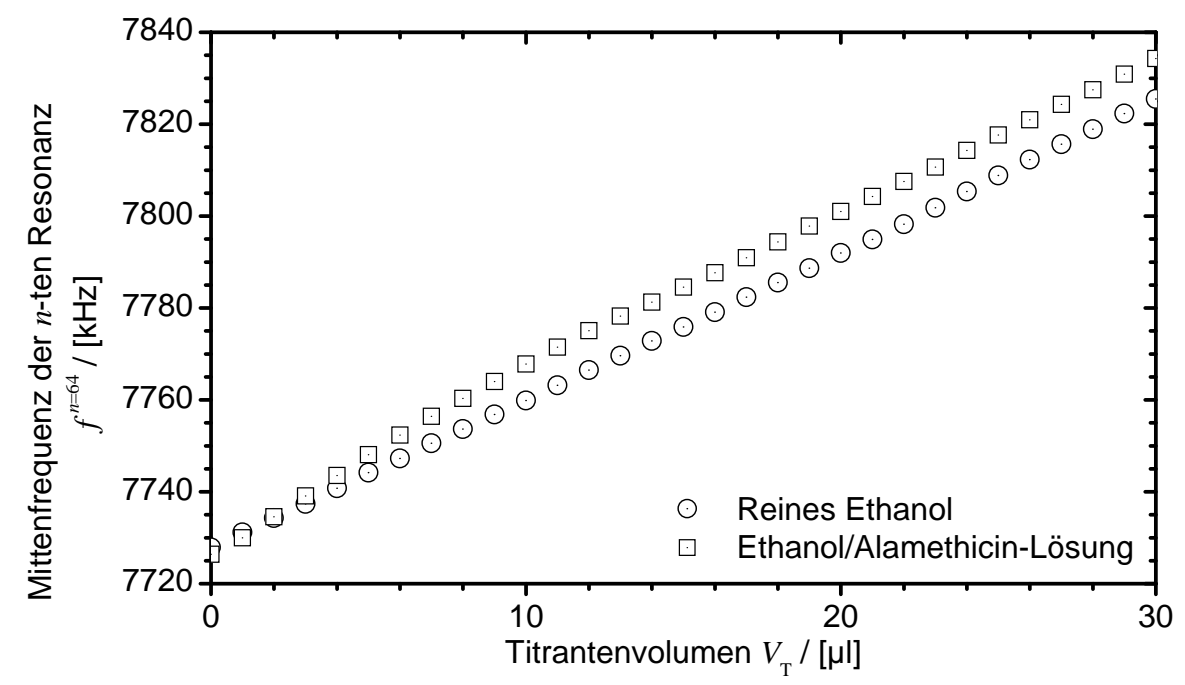

Abbildung 7.5: Darstellung der Resonanzfrequenz in Abhängigkeit von der Titrantenzugabe. In dieser Grafik ist der Verlauf der Resonanzfrequenz $f_{n=64}$ in Abhängigkeit von dem zugegeben Titrantenvolumen dargestellt. Dabei wurde zum einen reines Ethanol zu einer DMPCLösung hinzugegeben und zum anderen eine Ethanol/Alamethicin-Lösung gemessen.

In Abb. 7.5 ist die Mittenfrequenz der $n$-ten Zellresonanz exemplarisch für $n=64$ aufgetragen. Die Auftragung der direkten Messgrößen zeigt eine lineare Abhängigkeit der Mittenfrequenz von der Ethanolkonzentration. Die Zugabe von Alamethicin in Ethanol bewirkt einen zusätzlichen Anstieg der Mittenfrequenz bei geringen Alamethicinmengen. Abb. 7.6 zeigt die Auftragung der relativen Schallgeschwindigkeit $c_{\mathrm{s}}-c_{\mathrm{s}, \text { ref }}$ mit der Schallgeschwindigkeit von Alamethicin $c_{\mathrm{s}}$ und der Referenzschallgeschwindigkeit $c_{\mathrm{s}, \mathrm{ref}}$ von Ethanol für die folgenden Resonanzen

$$
f_{0}^{n=40}=4959 \mathrm{kHz} \quad f_{0}^{n=64}=7807 \mathrm{kHz} \quad f_{0}^{n=93}=11392 \mathrm{kHz} .
$$

Die Messung wurde bei $T=17,24 \pm 0,1^{\circ} \mathrm{C}$ in der gelförmigen Phase durchgeführt. Die Einflüsse von Temperaturschwankungen konnten durch gleichzeitige Resonanzmessung der mit Wasser gefüllten Zelle 2 korrigiert werden, indem die Differenz zu dieser Wasserreferenz gebildet wurde.

Die Auftragung der relativen Schallgeschwindigkeit, dargestellt in Abb. 7.6, zeigt drei Bereiche unterschiedlicher Steigung. Diese können mit Hilfe der Membrane Thinning-Theorie qualitativ erklärt werden. 


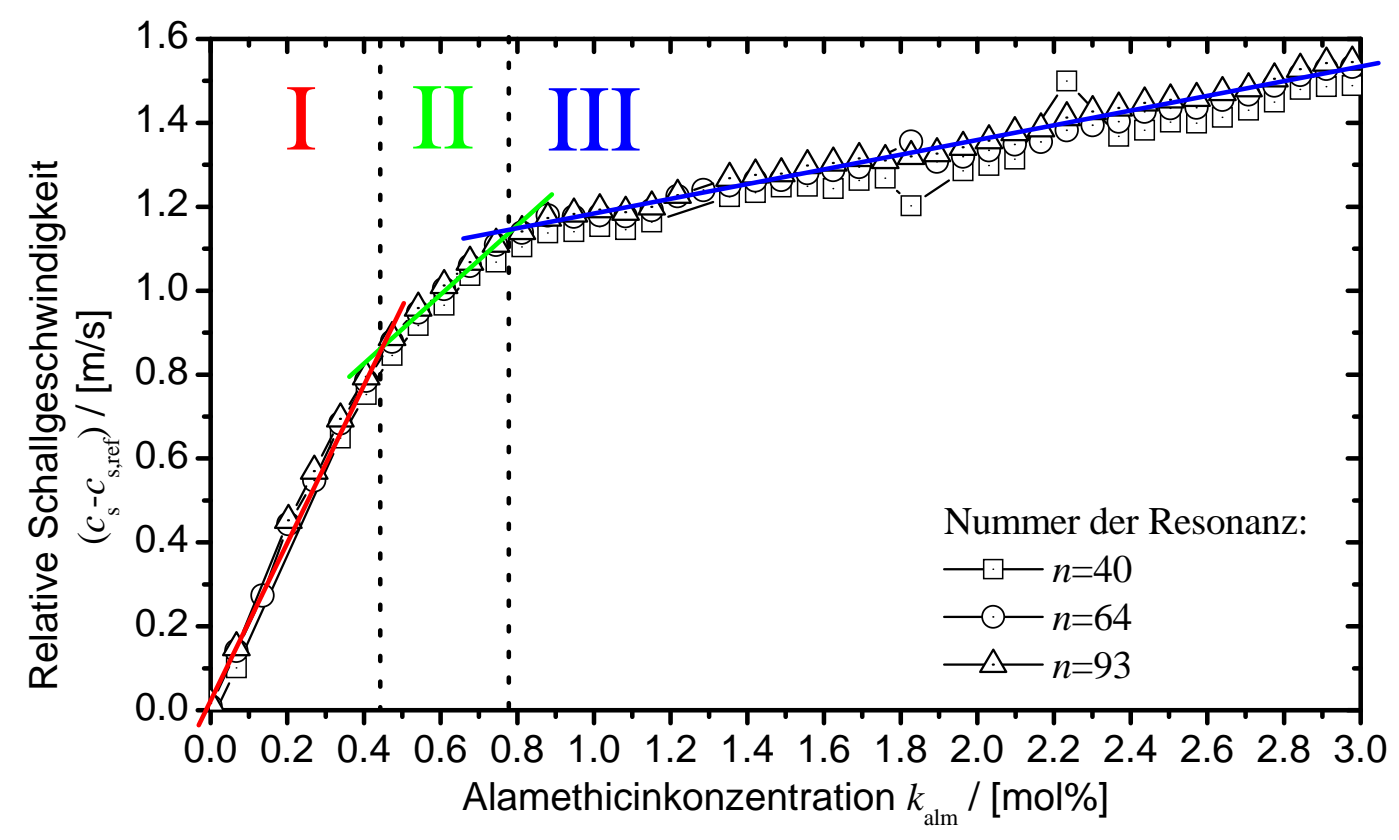

Abbildung 7.6: Änderung der Schallgeschwindigkeit bei Zugabe von Alamethicin. Der Graph zeigt die relative Schallgeschwindigkeit bei Zugabe von Alamethicin. Es lassen sich drei Bereiche unterschiedlicher Steigung erkennen.

Bereich I wird durch einen starken Anstieg der relativen Schallgeschwindigkeit charakterisiert. Nach der Membrane Thinning-Theorie wird hier die Adsorption an der Oberfläche vorhergesagt. Mit Hilfe der Titrationsvelocimetrie kann die Obergrenze dieses Bereiches mit $k_{\mathrm{alm}} \approx 0,45 \mathrm{~mol} \%$ angegeben werden.

Der Anstieg der Schallgeschwindigkeit kann nach $c_{\mathrm{s}}=\sqrt{1 /\left(\kappa_{\mathrm{S}} \rho\right)}$ auf die Verringerung der Kompressibilität zurückgeführt werden, da die Dichte der Lösung bei Zugabe von Alamethicin konstant bleibt. Für diesen Effekt können zwei mögliche Ursachen angeführt werden: die lokale Deformation und die Hydratation des Peptids an der Oberfläche. Die lokale Deformation führt, wie zuvor schon für die axiale Diffusion und Vesikeldeformation diskutiert, zu einer Neuordnung der Membranlipide und einer Erhöhung der inneren Membranspannung [59, 79, 101], welche einem äußeren komprimierenden Druck entgegenwirkt. Der zweite Effekt, die Hydratation, führt zur Stabilisierung der Alamethicin-Helix [108] (siehe auch Abschnitt 2.3). Dabei wird ein Wasserstoffbrückennetzwerk zwischen den hydrophilen Gruppen des Alamethicin und des Hydratwassers der Lipidmembran ausgebildet. Ein solches Netzwerk führt, wie Messungen an Ionenlösungen und Biomolekülen [22, 23, 24, 25] zeigen, ebenfalls zu einer Verringerung der Kompressibilität aufgrund der reduzierten Abstände zwischen benachbarten Molekülen.

In einen zweiten Konzentrationbereich sagt die Membrane Thinning-Theorie die Integration einzelner Peptide in die Membran voraus. Dieser Bereich liegt nach dieser Messung zwischen $0,45<k_{\text {alm }}<0,75 \mathrm{~mol} \%$. Hier sind zwei gegenläufige Effekte zu betrachten, die eine Reduzierung des ursprünglichen Anstiegs bewirken. Durch die Integration des Peptids in die Membran wird diese stabilisiert, wie auch die deutliche Reduktion der kritischen Fluktuationen nahe legt (siehe Abschnitt 6.2.1). Die Stabilisierung führt zu einer Verringerung der Kompressibilität. Gleichzeitig wird durch die Integration die be- 
stehende Hydratisierung des zuvor adsorbierten Alamethicins aufgehoben und somit die Kompressibilität erhöht. Die Erhöhung der Schallgeschwindigkeit zeigt, dass der Prozess der Integration den dominanten Beitrag zur Kompressibilität liefert.

Im dritten Konzentrationsbereich oberhalb $k_{\text {alm }} \geq 0,75 \mathrm{~mol} \%$ ist nur noch ein sehr geringer Anstieg der Schallgeschwindigkeit zu beobachten. Es wird kein Peptid mehr an der Oberfläche adsorbiert. Die weitere Erhöhung der Alamethicin konzentration führt zu der Komplexierung des integrierten Peptids. Dabei werden Poren geformt, die einen leicht komprimierbaren Hohlraum umschließen. Es ist aber gleichzeitig durch die weitere Integration von Alamethicin in die Membran mit fortschreitender Stabilisierung zu rechnen, so dass sich die Kompressibilitätsänderungen durch Hohlraumbildung und Membranstabilisierung zu einem großen Teil kompensieren.

Die Titrationsexperimente konnten leider nur in einem sehr engem Zeitrahmen bei Ultrasonic Scientific Ltd. in Dublin durchgeführt werden. Mehrfachmessungen an diesem System waren daher leider nicht möglich. Die Erklärungen für den qualitativen Verlauf der Kurve beruhen auf den zuvor gemachten Ergebnisse aus der Spektrometrie und der Membrane Thinning-Theorie, können jedoch anhand der vorliegenden Daten nicht eindeutig belegt werden. 


\section{Zusammenfassung und Ausblick}

In dieser Arbeit wurde erstmals das Verhalten von peptidhaltigen Lipidmembranen während des Phasenübergangs von der gelförmigen Phase in die fluide Phase mit Hilfe der Ultraschallabsorptionsspektrometrie untersucht. Dazu wurden als Modellsystem unilamellare Vesikel aus einer DMPC-Doppelschicht verwendet, die mit dem porenbildenden Peptid Alamethicin versetzt wurden.

Der Phasenübergang 2. Ordnung wird unter anderem durch Domänenbildung und Fluktuationen von thermodynamischen Ordnungsparametern wie z. B. der Dichte bestimmt. Es wird ein Anstieg der Fluktuationen bei Annäherung an die Übergangstemperatur beobachtet, der durch ein Potenzgesetz beschrieben wird. Dieses bezeichnet man auch als kritisches Verhalten.

Das Messverfahren der Ultraschallspektrometrie nutzt als „Sonde“ die Veränderungen des Lipidvolumens während des Phasenübergangs. Für die Messungen des kritischen Verhaltens wurde ein neuer Messplatz nach dem Resonatorverfahren aufgebaut, der sowohl die Anforderungen an die Messgenauigkeit erfüllt, als auch aufgrund eines geringen Messvolumens die Kosten für die Messungen minimiert. Für die Spektrometrie im Frequenzbereich zwischen $100 \mathrm{kHz}$ und $10 \mathrm{MHz}$ werden zwei Resonatoren verwendet, die an einem Netzwerkanalysator betrieben werden. Ein Steuerprogramm mit einem adaptiven ScanAlgorithmus optimiert die notwendigen Messparameter über einen Dynamikbereich von $80 \mathrm{~dB}$ und für Resonanzbreiten zwischen $1 \mathrm{~Hz}$ und $10000 \mathrm{~Hz}$. Zudem wird der Messplatz auch für die temperaturabhängige Ultraschallvelocimetrie genutzt.

Die dynamische Skalierungstheorie von Bhattacharjee und Ferrell beschreibt die Beiträge von Dichtefluktuationen im Ultraschallabsorptionsspektrum. Aus Messungen an reinen DMPC-Membranen und cholesterolhaltigen Membranen ist bekannt, dass neben dem kritischen Anteil auch Beiträge aus Einzelzeitrelaxationen zu berücksichtigen sind, die mit der axialen Diffusion des Lipids und der Rotationsisomerierung der Alkylketten des Lipids erklärt werden. In der Nähe der Phasenumwandlung tritt ein zusätzlicher Term auf, der mit lokalen Deformationen der Vesikel in Verbindung gebracht wird. Die Spektren alamethicinhaltiger Membranen lassen sich ebenfalls mit Hilfe dieser Terme beschreiben. Jedoch treten deutliche Abweichungen in Amplitude und Relaxationszeit der Beiträge auf.

Anhand der gemessenen Skalierungsfunktion wurde bestätigt, dass sich die kritischen Beiträge nach Bhattacharjee und Ferrell beschreiben lassen. Es stellte sich heraus, dass der Effekt der kritischen Verlangsamung in Anwesenheit von Alamethicin verstärkt wird. Die Abschätzung über die Verknüpfung von Korrelationslänge und Relaxationsrate für das Einsetzen des kritischen Verhaltens deutet darauf hin, dass das kritische Verhalten mit steigendem Alamethicingehalt bei niedrigeren Temperaturen einsetzt.

Die Integration von Alamethicin findet nach der Membrane Thinning-Theorie in drei 
unterschiedlichen Konzentrationsbereichen statt, deren Grenzen durch die Titrationsvelocimetrie bestimmt wurden. Für Konzentrationen unterhalb $0,45 \mathrm{~mol} \%$ wird Alamethicin auf der Membranoberfläche adsorbiert, wobei die Moleküle statistisch verteilt sind. Oberhalb dieses Bereiches findet eine Integration des Alamethicin in die Membran statt. Für Konzentrationen oberhalb 0,75 mol\% beginnt Alamethicin Komplexe und damit Poren zu bilden.

In allen Konzentrationsbereichen wird eine Verlangsamung der Fluktuationen durch Alamethicin beobachtet, die sich mit der Membrane Thinning-Theorie erklären lässt. Die Einlagerung von Alamethicin in die Kopfgruppenregion führt zu einer Einschränkung der lateralen Diffusion der Lipide und damit auch zu einer Verlangsamung der Fluktuationen. Um die Volumenvergrößerung der Membran durch das Einbringen des Peptids zu kompensieren, wird die Membran lokal aufgeschmolzen. Die Volumenerhaltung bei verschiedenen Alamethicinkonzentrationen wurde mit der Densitometrie bestätigt.

Nach dem Einsetzen der Integration bleibt die mittlere Membrandicke auf einem Minimum, d. h. die Anzahl der lokalen Deformationen bleibt konstant. Das integrierte Alamethicin bildet Bindungen zu den Lipidkopfgruppen in beiden Membranschichten aus und führt über diesen Mechanismus schon bei geringen Konzentrationen zu einer deutlichen Verlangsamung der kritischen Relaxationsrate.

Für den Spektralterm der axialen Diffusion wurde eine Verringerung der Amplitude und eine Erhöhung der Relaxationszeit durch Alamethicin mittels Ultraschallabsorptionsspektrometrie nachgewiesen. Da die axiale Diffusion eng mit der lateralen Diffusion verknüpft ist, ist dieser Effekt ebenfalls auf die lokale Deformation zurückzuführen. Es wurde gezeigt, dass eine Proportionalität zwischen der Amplitude des Spektralterms und der mittleren Membrandicke besteht. Im Vergleich zum reinen DMPC ist beim alamethicinhaltigen System eine ausgeprägtere Verlangsamung der Relaxationszeiten in der Nähe des Phasenübergangs zu beobachten. Dies lässt darauf schließen, dass die axiale Diffusion in Anwesenheit von Alamethicin stärker an das kritische Verhalten koppelt.

Besonders die Parameter des Spektralterms der Vesikeldeformation zeigen eine deutliche Abhängigkeit von der Alamethicinkonzentration. Die Amplitude des Terms steigt mit der Konzentration deutlich an. Die durch die Deformation zusätzlich erzeugte Membranspannung wurde mit der lysierenden Wirkung des Alamethicins in Verbindung gebracht. Die Relaxationszeiten der Vesikeldeformation zeigen ebenso wie die der axialen Diffusion in Anwesenheit von Alamethicin eine stärkere Kopplung an das kritische Verhalten.

Wie die Messungen aus Ultraschallspektrometrie und Titrationsvelocimetrie zeigten, ist schon bei einem niedrigen Alamethicingehalt mit der Integration des Peptids zu rechnen und womit auch das Minimum der Membrandicke erreicht wird. Zukünftige Untersuchungen sind daher bei geringen Alamethicinkonzentrationen durchzuführen, um eine Beziehung zwischen der Membrandicke und dem kritischen Verhalten am Phasenübergang zu herstellen zu können.

Um eine gute Anpassung der kritischen Parameter zu erzielen, ist die Kenntnis der kritischen Temperatur von großer Wichtigkeit. Diese sollte in Zukunft mit der differenziellen Kalorimetrie, die im Rahmen dieser Arbeit leider nicht zur Verfügung stand, bestimmt werden. Die Kalorimetrie erlaubt darüber hinaus die Untersuchung weiterer thermodynamischer Parameter in der Nähe des Phasenübergangs. 


\section{A. Darstellung der Ultraschallspektren}

\section{A.1. DMPC:Alamethicin, 99,7:0,3 mol\%}
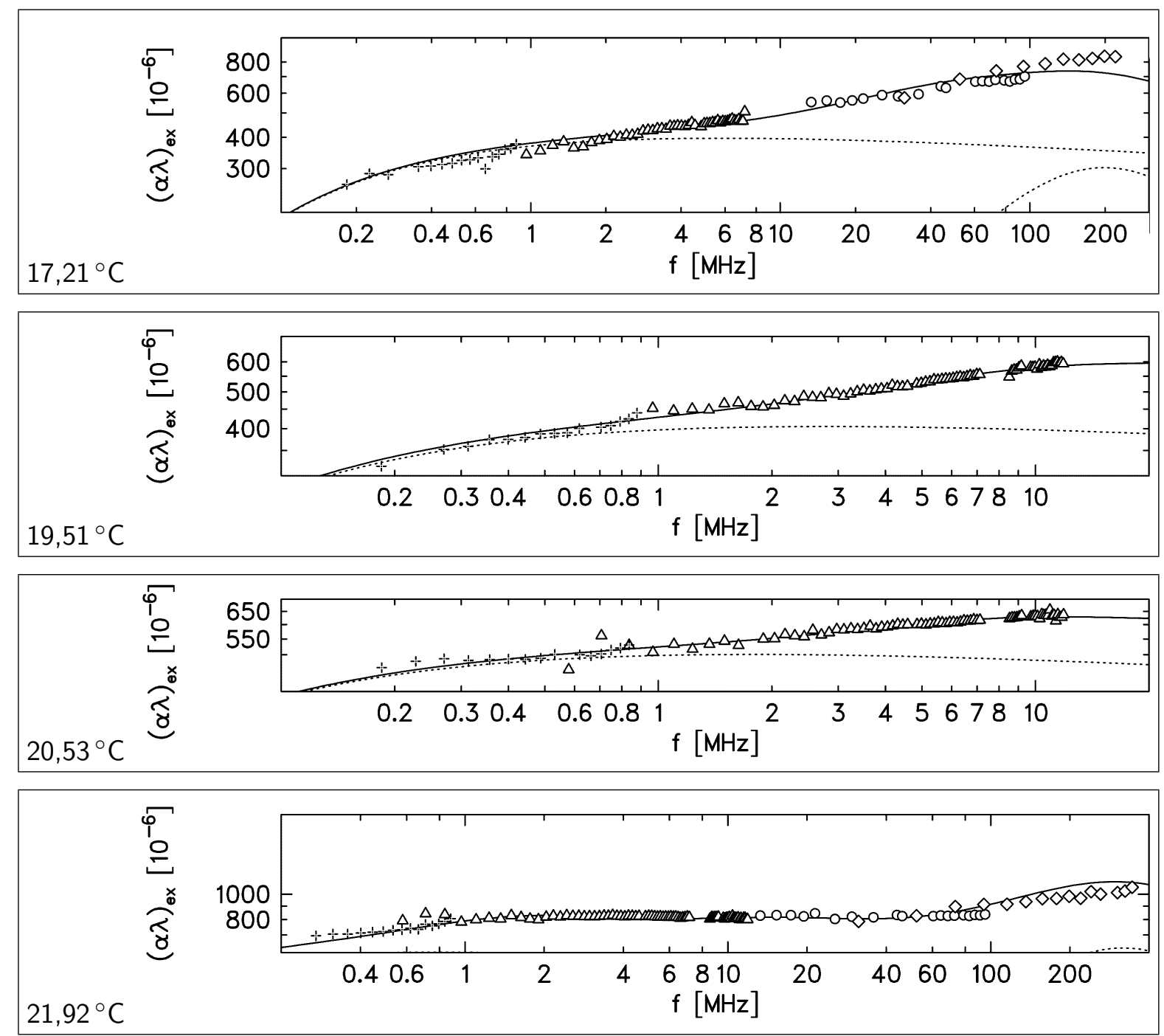

Abbildung A.1: Absorptionsspektren von DMPC:Alm 99,7:0,3 mol\%. Symbole für Apparaturen: +: 1-MHz-Reflektor-Resonator; $\triangle$ : 8-MHz-Resonator; 0 : tieffrequente Pulszelle; $\diamond$ : hochfrequente Pulszelle. Die durchgezogene Linie stellt die Summe der Beiträge der Spektralterme dar, die gestrichelte die Beiträge der einzelnen Terme. 
A. Darstellung der Ultraschallspektren
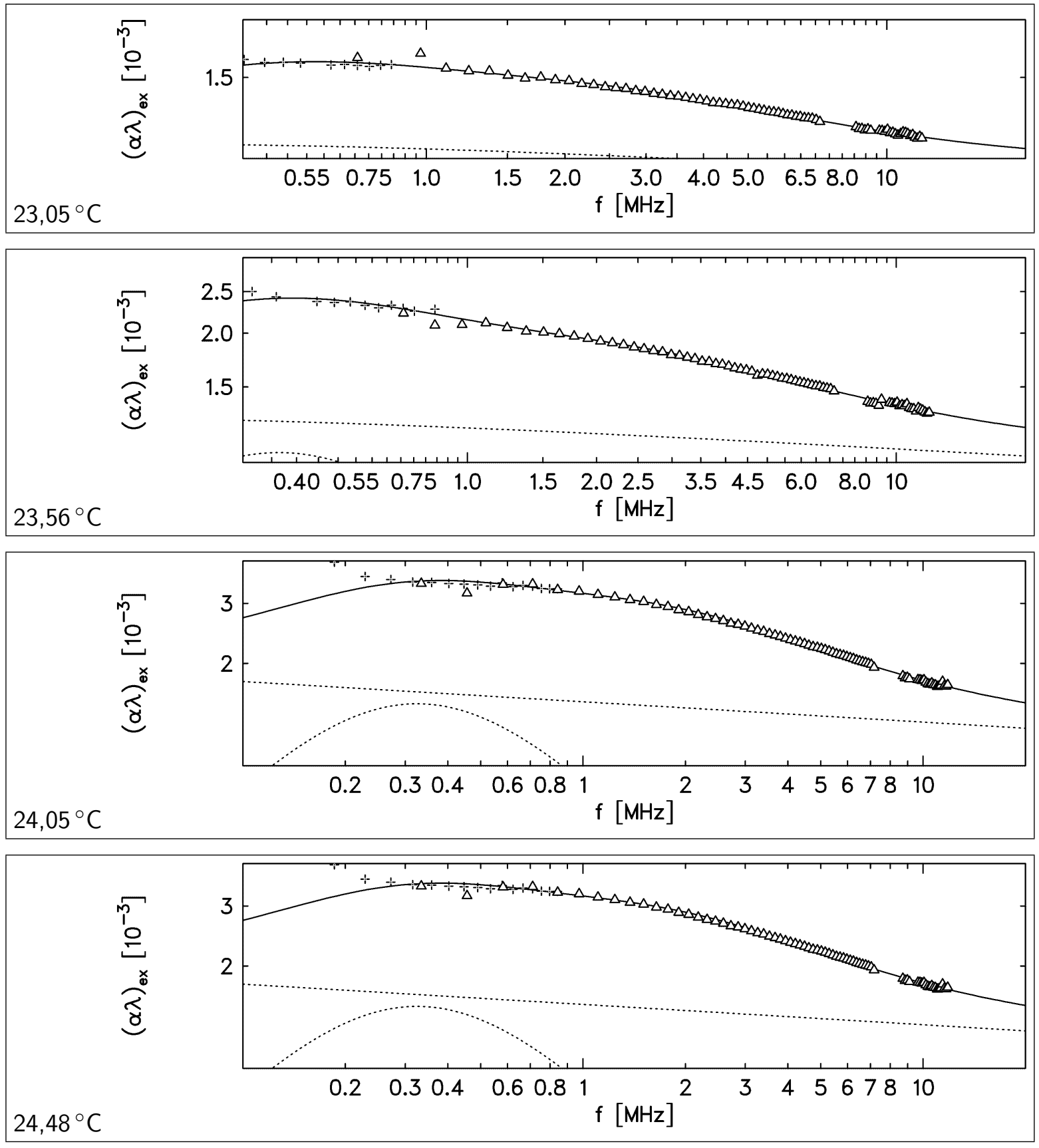

Abbildung A.2: Absorptionsspektren von DMPC:Alm 99,7:0,3 mol\%. Symbole für Apparaturen: +: 1-MHz-Reflektor-Resonator; $\triangle:$ 8-MHz-Resonator. Die durchgezogene Linie stellt die Summe der Beiträge der Spektralterme dar, die gestrichelte die Beiträge der einzelnen Terme. 

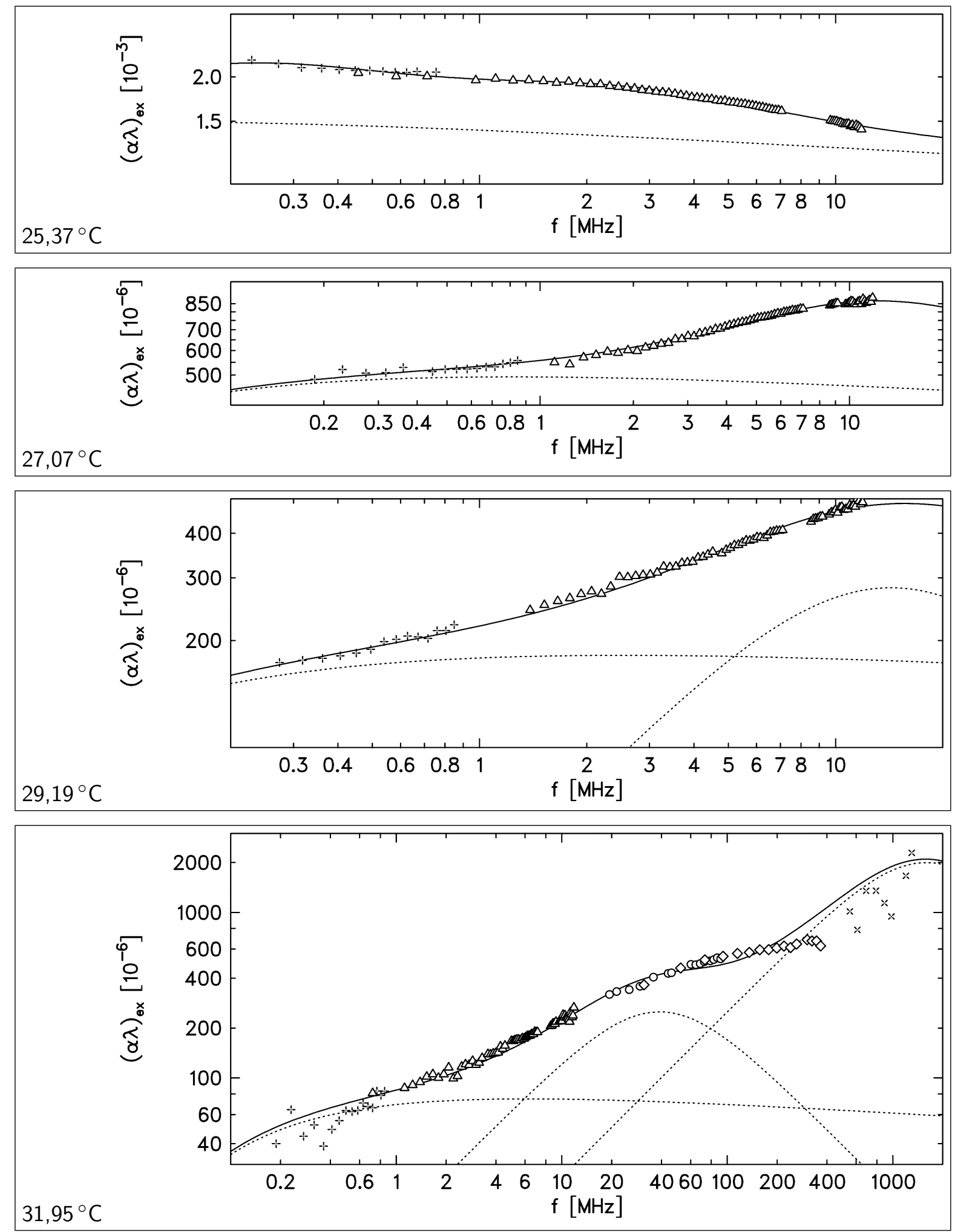

Abbildung A.3: Absorptionsspektren von DMPC:Alm 99,7:0,3 mol\%. Symbole für Apparaturen: +: 1-MHz-Reflektor-Resonator; $\triangle$ : 8-MHz-Resonator; $\circ$ : tieffrequente Pulszelle; $\diamond$ : hochfrequente Pulszelle; $x$ : tieffrequente Hyperschallzelle. Die durchgezogene Linie stellt die Summe der Beiträge der Spektralterme dar, die gestrichelte die Beiträge der einzelnen Terme. 
A. Darstellung der Ultraschallspektren

\section{A.2. DMPC:Alamethicin, 99,5:0,5 mol\%}
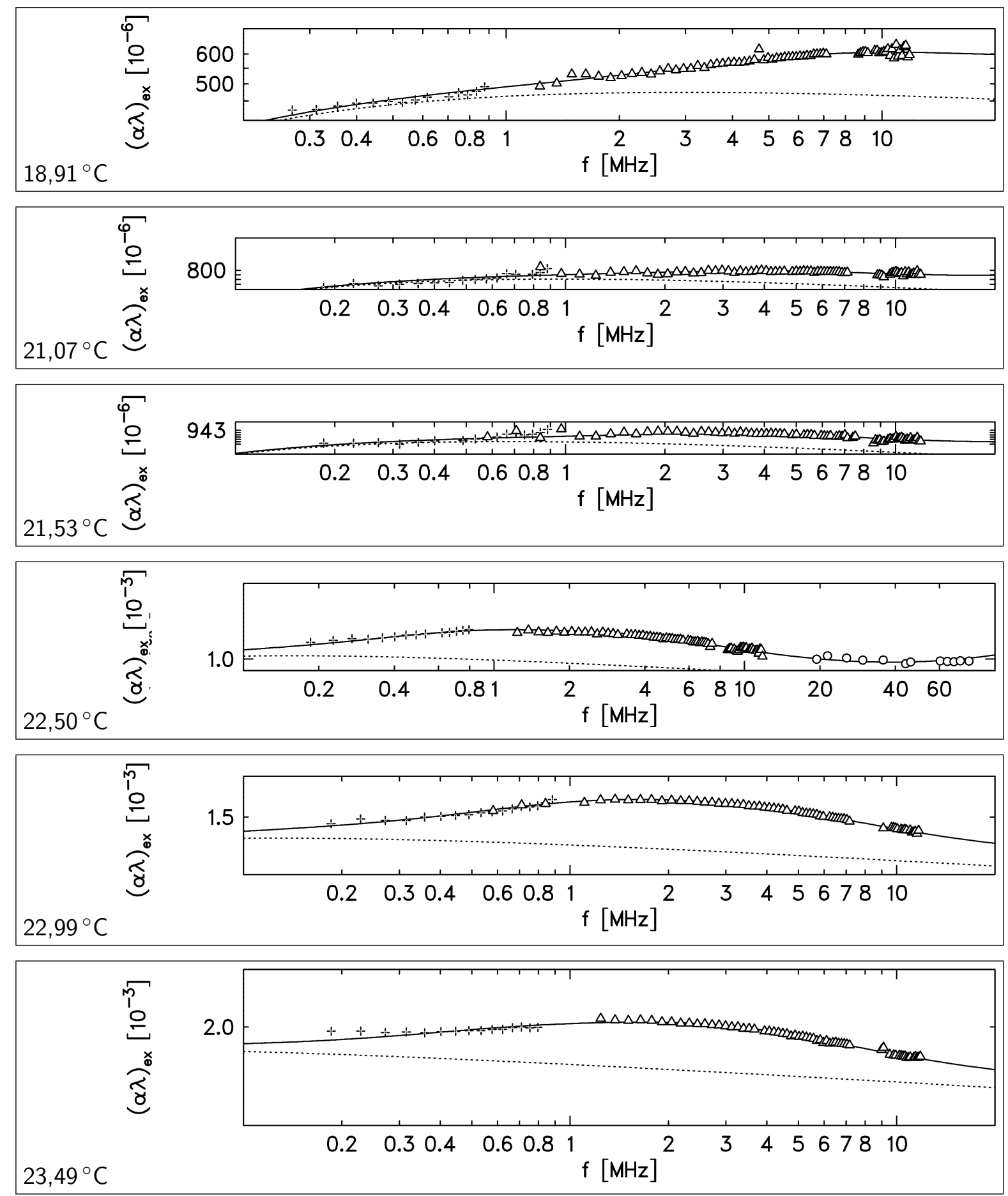

Abbildung A.4: Absorptionsspektren von DMPC:Alm 99,5:0,5 mol\%. Symbole für Apparaturen: +: 1-MHz-Reflektor-Resonator; $\triangle$ : 8-MHz-Resonator; o: tieffrequente Pulszelle. Die durchgezogene Linie stellt die Summe der Beiträge der Spektralterme dar, die gestrichelte die Beiträge der einzelnen Terme. 

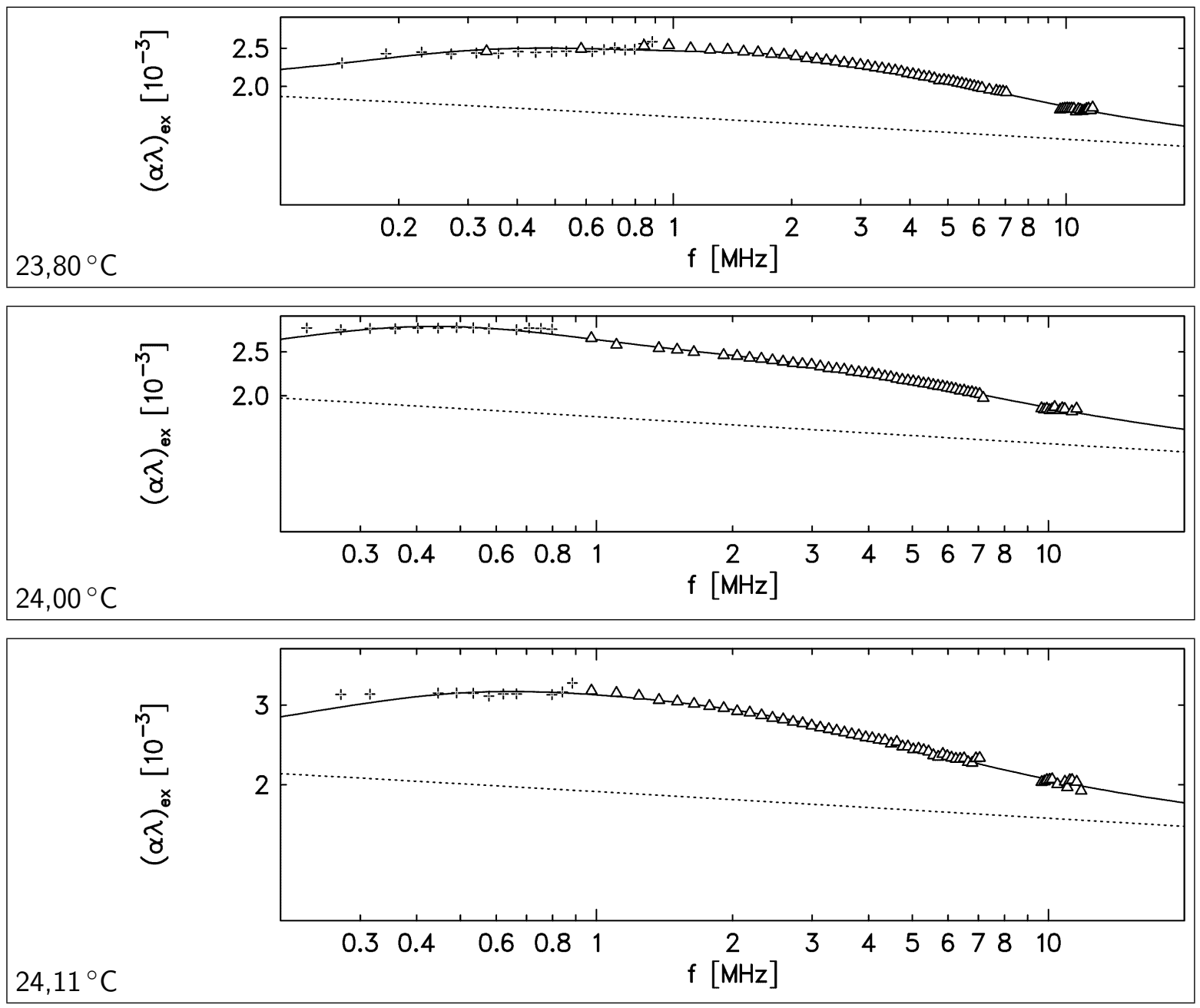

Abbildung A.5: Absorptionsspektren von DMPC:Alm 99,5:0,5 mol\%. Symbole für Apparaturen: +: 1-MHz-Reflektor-Resonator; $\triangle:$ 8-MHz-Resonator. Die durchgezogene Linie stellt die Summe der Beiträge der Spektralterme dar, die gestrichelte die Beiträge der einzelnen Terme. 


\section{A.3. DMPC:Alamethicin, 99,3:0,7 mol\%}
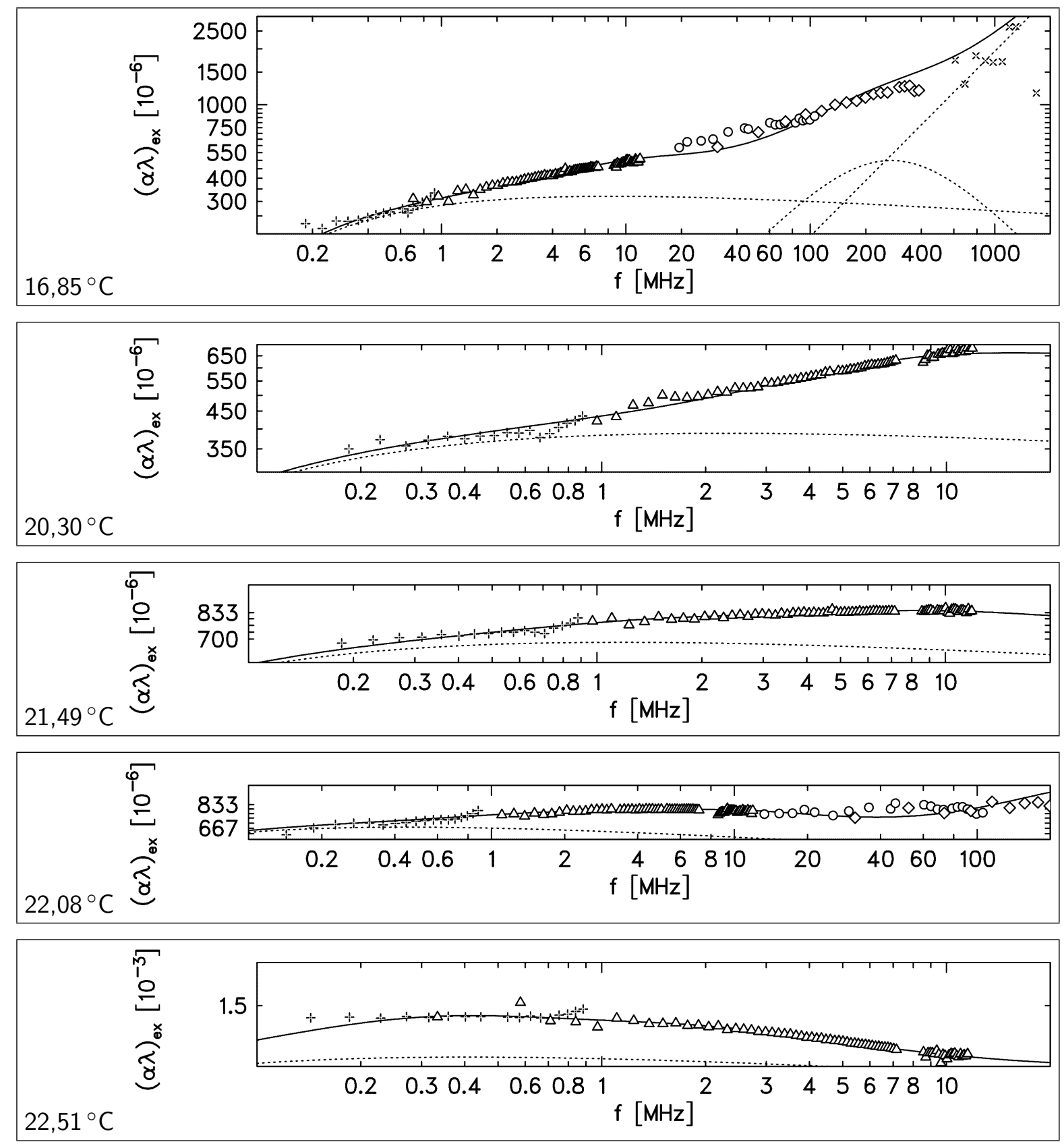

Abbildung A.6: Absorptionsspektren von DMPC:Alm 99,3:0,7 mol\%. Symbole für Apparaturen: +: 1-MHz-Reflektor-Resonator; $\triangle$ : 8-MHz-Resonator; 0 : tieffrequente Pulszelle; $\diamond$ : hochfrequente Pulszelle; $\times$ : tieffrequente Hyperschallzelle. Die durchgezogene Linie stellt die Summe der Beiträge der Spektralterme dar, die gestrichelte die Beiträge der einzelnen Terme. 

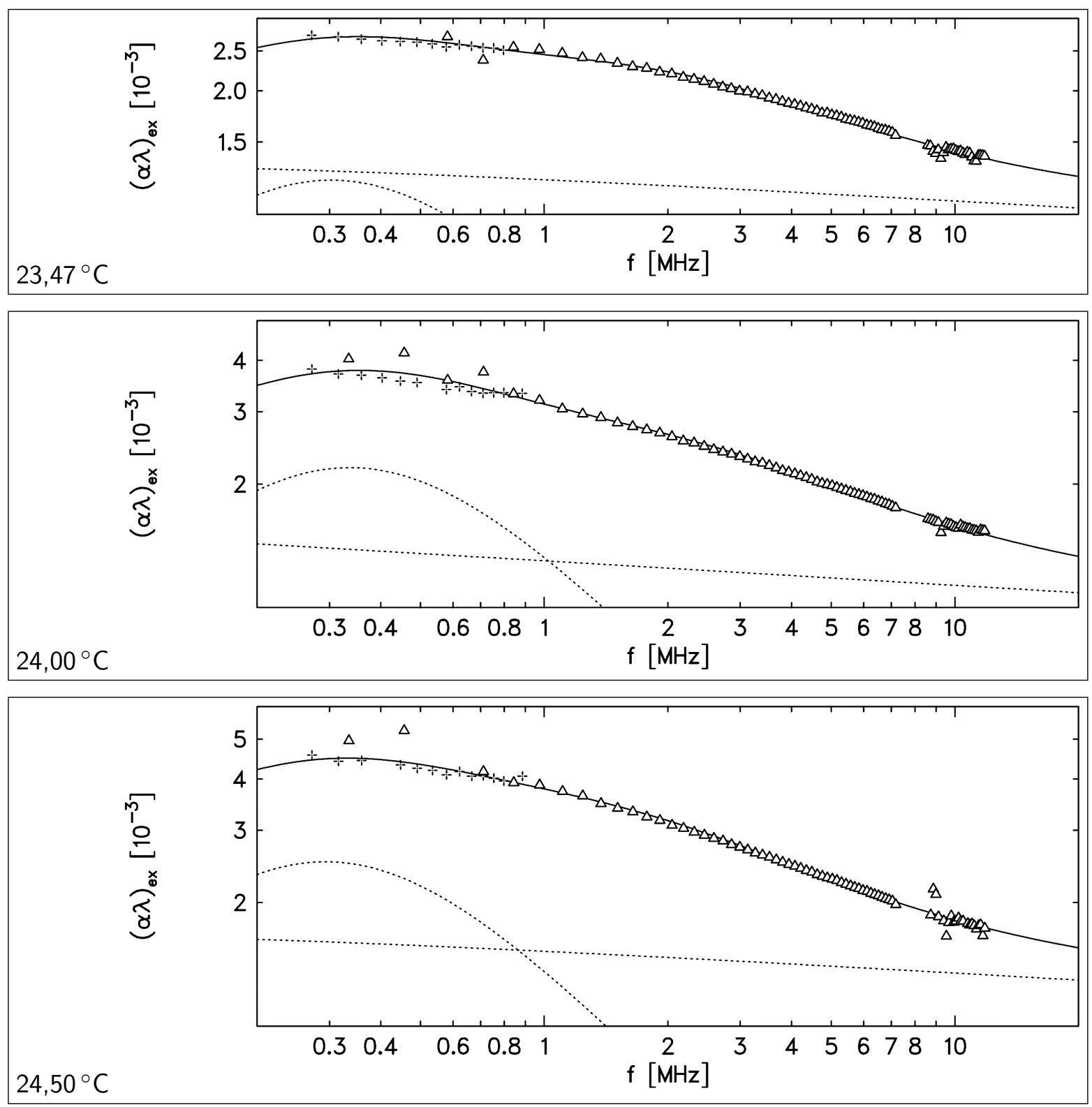

Abbildung A.7: Absorptionsspektren von DMPC:Alm 99,3:0,7 mol\%. Symbole für Apparaturen: +: 1-MHz-Reflektor-Resonator; $\triangle$ : 8-MHz-Resonator. Die durchgezogene Linie stellt die Summe der Beiträge der Spektralterme dar, die gestrichelte die Beiträge der einzelnen Terme. 
A. Darstellung der Ultraschallspektren
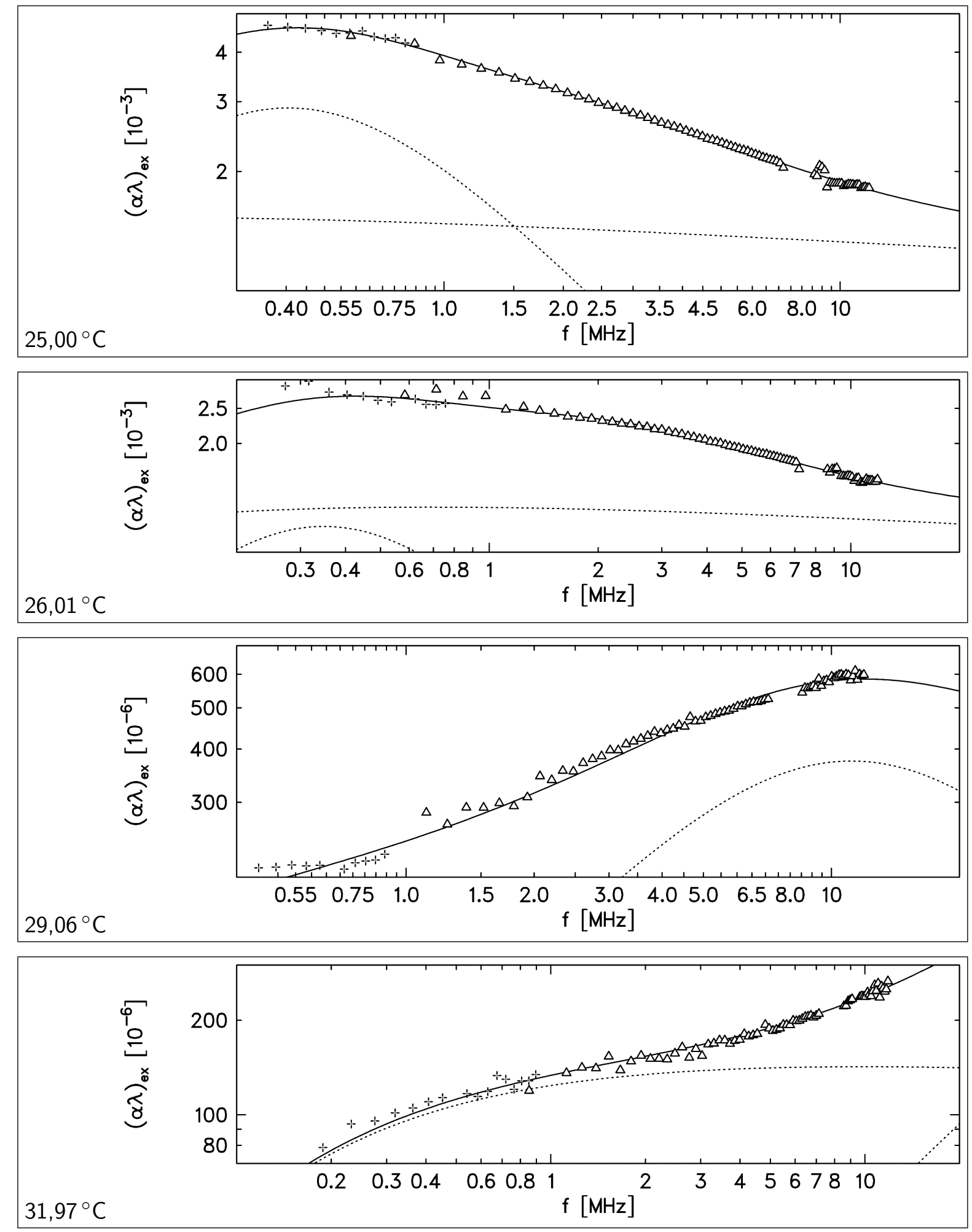

Abbildung A.8: Absorptionsspektren von DMPC:Alm 99,3:0,7 mol\%. Symbole für Apparaturen: +: 1-MHz-Reflektor-Resonator; $\triangle$ : 8-MHz-Resonator. Die durchgezogene Linie stellt die Summe der Beiträge der Spektralterme dar, die gestrichelte die Beiträge der einzelnen Terme. 


\section{A.4. DMPC:Alamethicin, 99:1 mol\%}
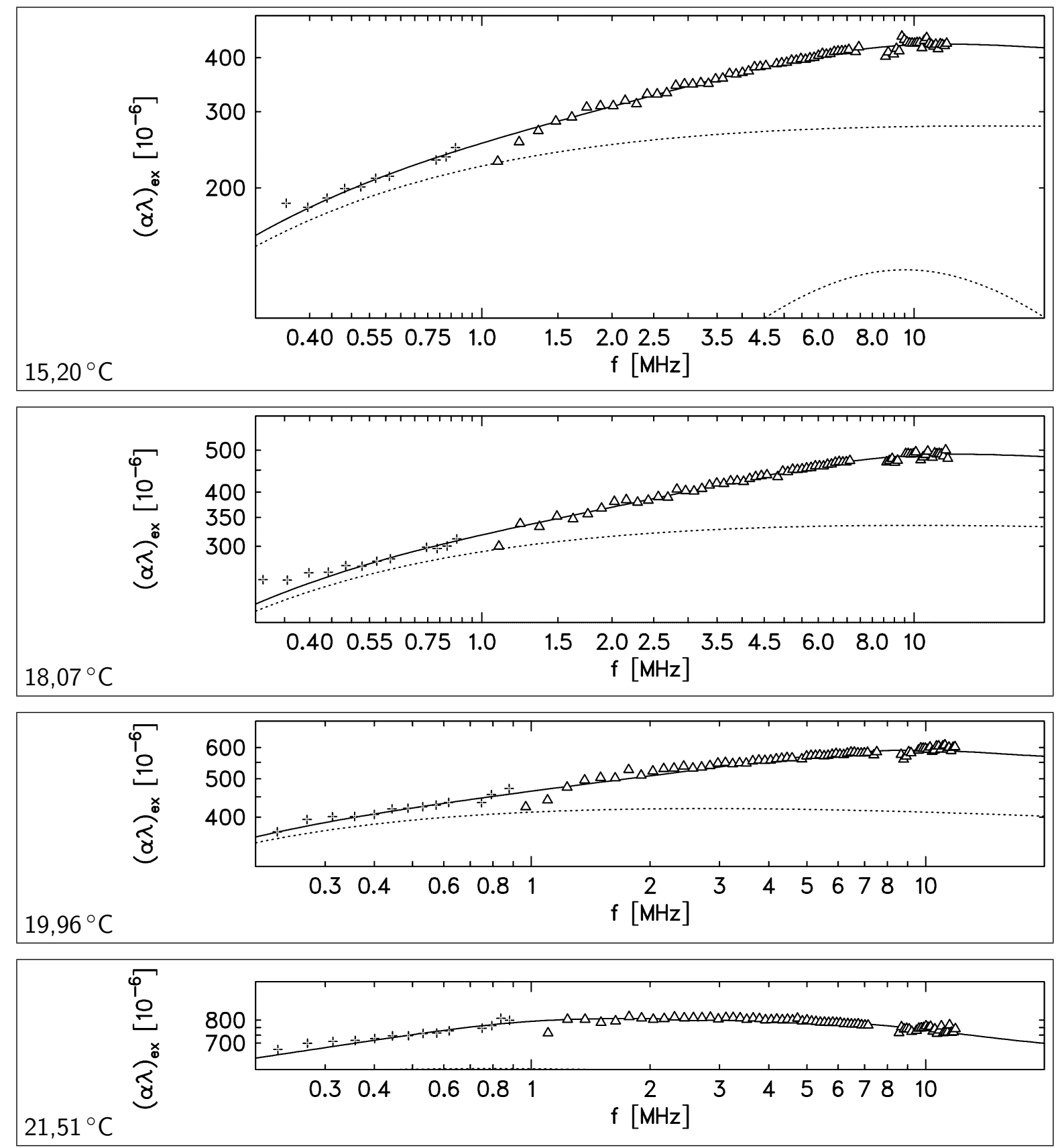

Abbildung A.9: Absorptionsspektren von DMPC:Alm 99:1 mol\%. Symbole für Apparaturen: +: 1-MHz-Reflektor-Resonator; $\triangle$ : 8-MHz-Resonator. Die durchgezogene Linie stellt die Summe der Beiträge der Spektralterme dar, die gestrichelte die Beiträge der einzelnen Terme. 

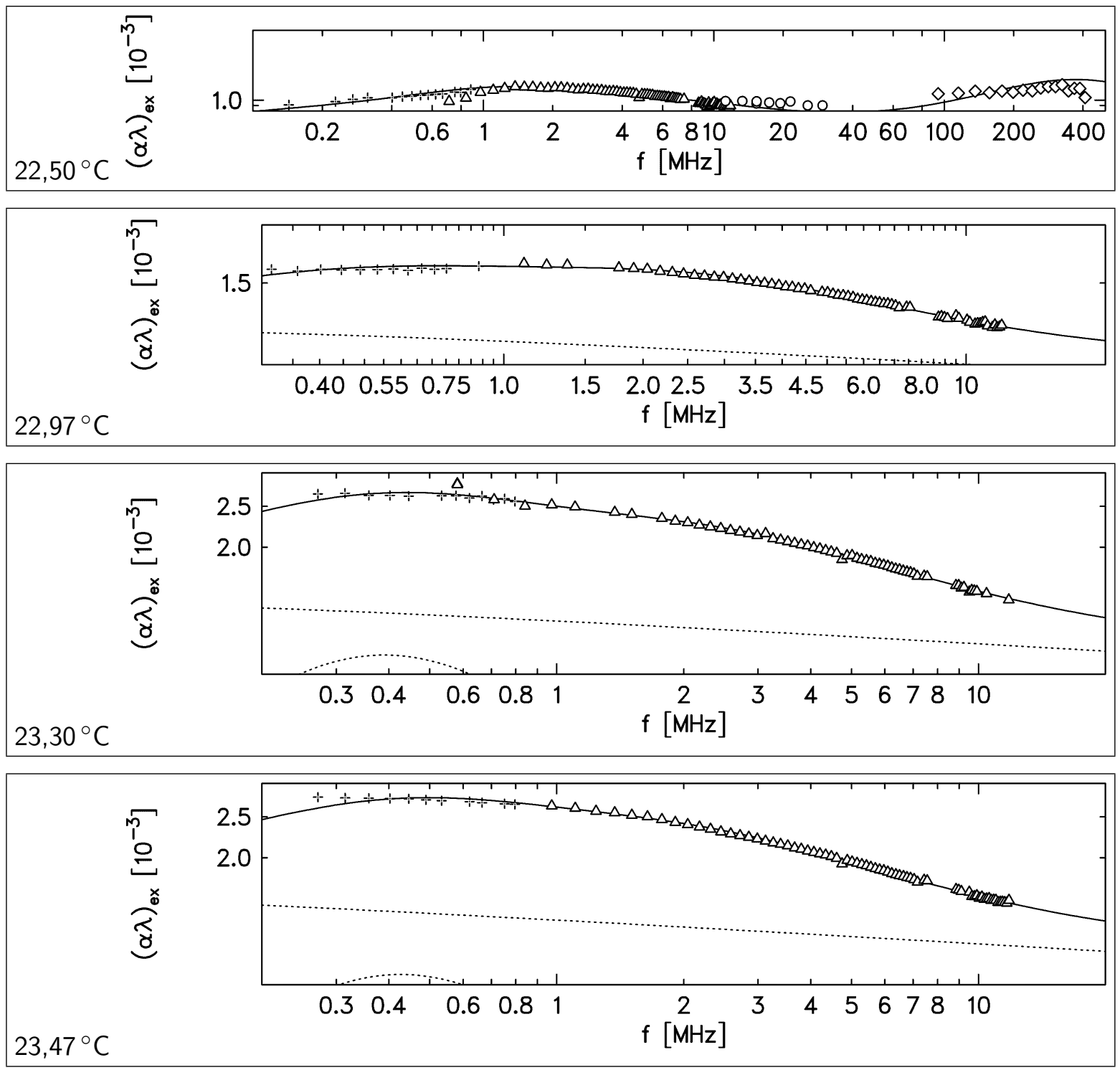

Abbildung A.10: Absorptionsspektren von DMPC:Alm 99:1 mol\%. Symbole für Apparaturen: +: 1-MHz-Reflektor-Resonator; $\triangle:$ : $\mathrm{MHz}$-Resonator; ०: tieffrequente Pulszelle; $\diamond$ : hochfrequente Pulszelle. Die durchgezogene Linie stellt die Summe der Beiträge der Spektralterme dar, die gestrichelte die Beiträge der einzelnen Terme. 


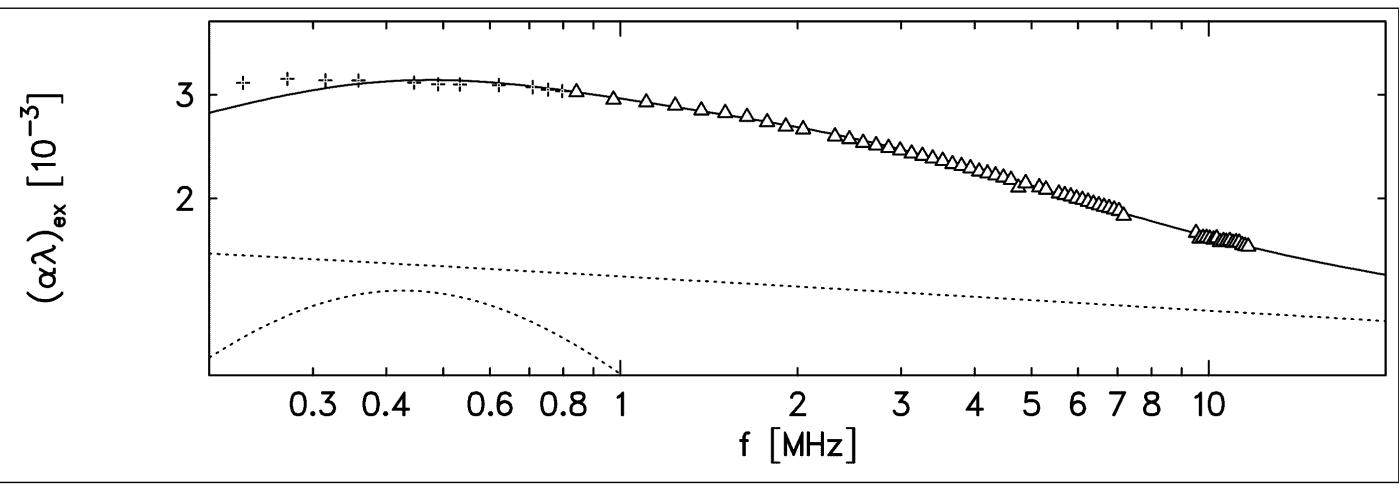

$23,60^{\circ} \mathrm{C}$

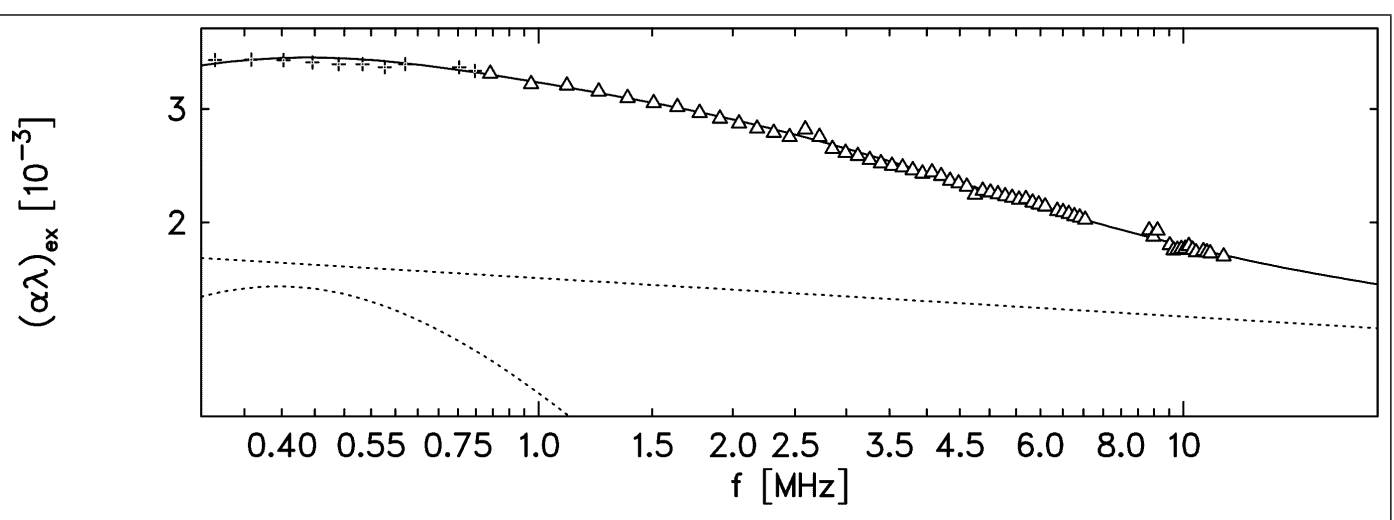

Abbildung A.11: Absorptionsspektren von DMPC:Alm 99:1 mol\%. Symbole für Apparaturen: +: 1-MHz-Reflektor-Resonator; $\triangle$ : 8-MHz-Resonator. Die durchgezogene Linie stellt die Summe der Beiträge der Spektralterme dar, die gestrichelte die Beiträge der einzelnen Terme. 
A. Darstellung der Ultraschallspektren 


\section{B. Bildschirmfotos von NWAControl}

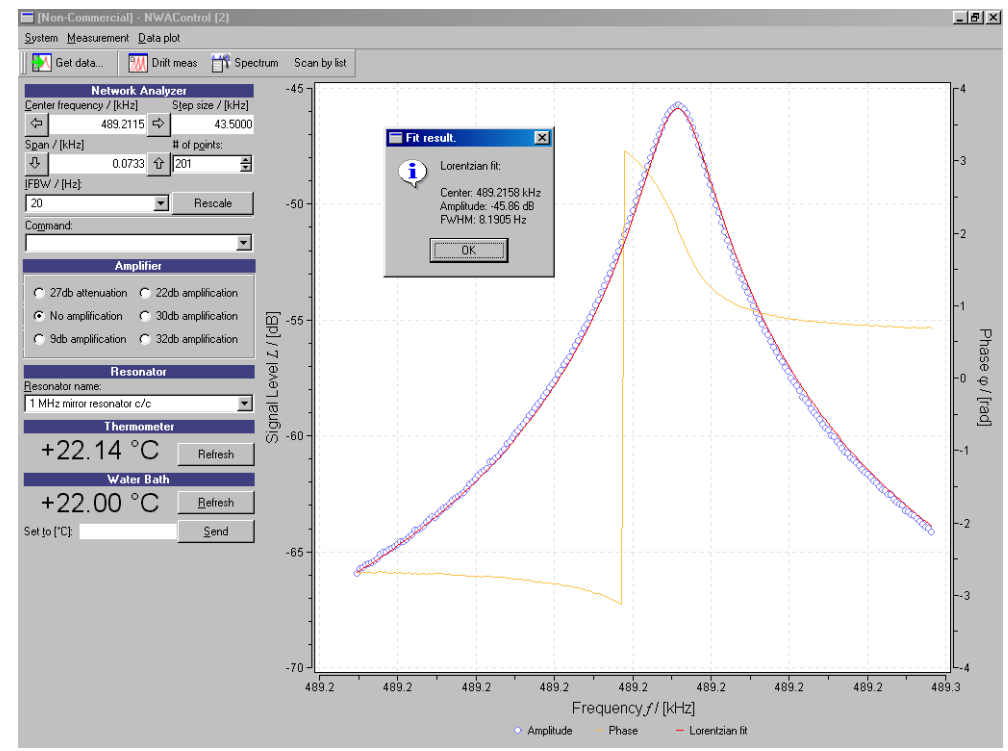

Abbildung B.1: Bildschirmfoto des Hauptfensters. Auf der linken Seite sind die Kontrollelemente für den Netzwerkanalysator, Verstärker, Thermometer und Wasserbad dargestellt. Das rechte Diagramm zeigt die Daten einer Resonanz überlagert mit einer Lorentzanpassung. Die hellblauen Kreise symbolisieren Datenpunkte der Amplitude, die orange Linie die Phaseninformation, die rote Kurve die Lorentzanpassung. Die Parameter der Lorentzanpassung werden in einem Popup-Fenster dargestellt.

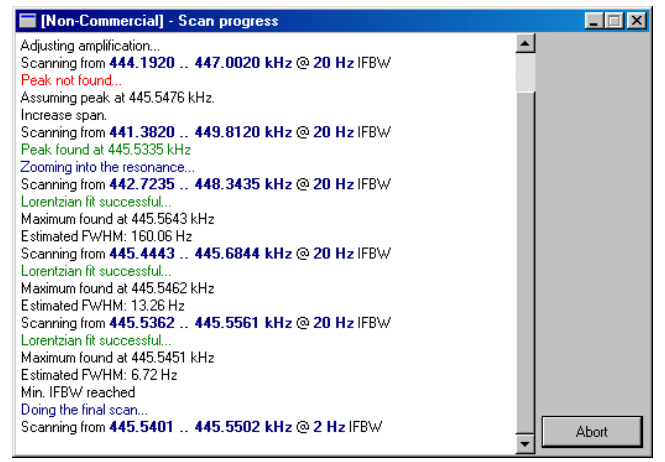

Abbildung B.2: Bildschirmfoto des Scan-Dialogs. Dieser Dialog gibt den Fortschritt des adaptiven Scan-Algorithmus an. Wie man sieht, wurde zuerst automatisch die Verstärkung und anschließend der Span angepasst, bevor schrittweise der Scan-Bereich um die Mittenfrequenz verkleinert wurde. Das Beispiel veranschaulicht weiterhin, dass sich mit reduziertem Span auch die Halbwertsbreite deutlich ändert. Vor dem "finalen" Scan wurde die kleinste IFBW erreicht, somit wurde ein Abbruchkriterium des Suchalgorithmus erfüllt. 


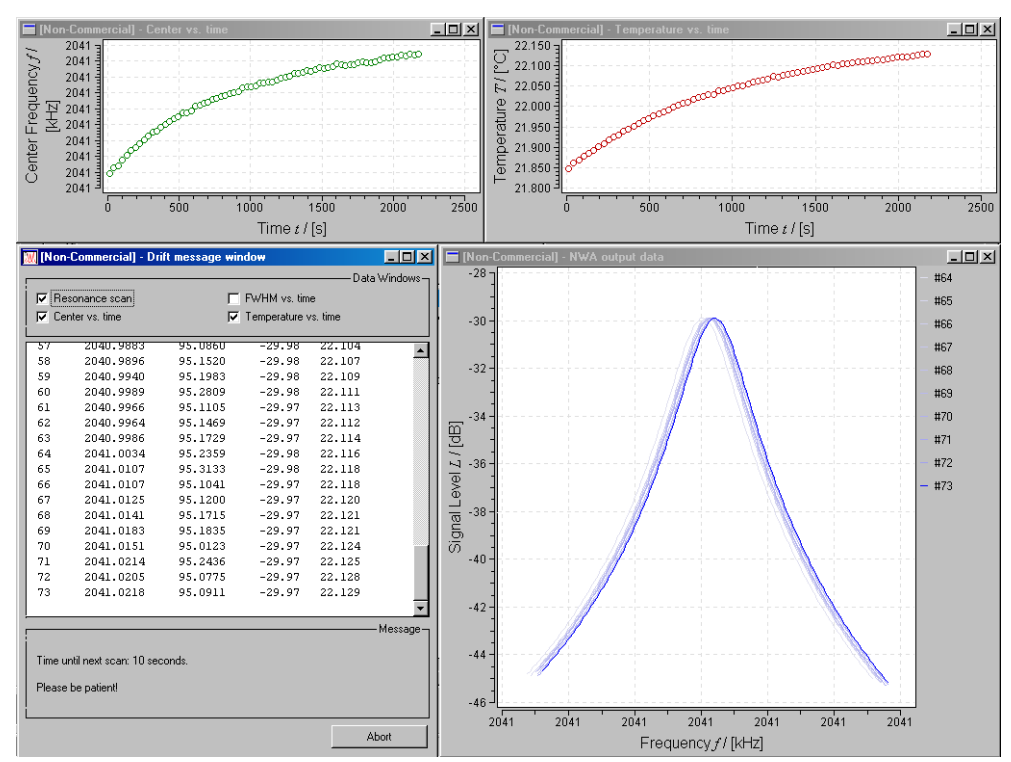

Abbildung B.3: Bildschirmfoto während einer Driftmessung. Dieses Bildschirmfoto zeigt Fenster während einer Driftmessung. Links oben ist der Verlauf der Mittenfrequenz zu erkennen, rechts oben ist der Temperaturverlauf gegenüber der Zeit aufgetragen. Man sieht, dass sich die Werte asymptotisch einer Horizontalen nähern. Das Fenster links unten enthält eine Tabelle mit den Messwerten über Mittenfrequenz, Halbwertsbreite und Temperatur. In dem rechten unteren Fenster werden die Messdaten der letzten zehn gemessenen Resonanzen dargestellt. Die Gesamtheit dieser Informationen bietet die Möglichkeit, die Stabilisierung von Mittenfrequenz und Temperatur gut abzuschätzen.

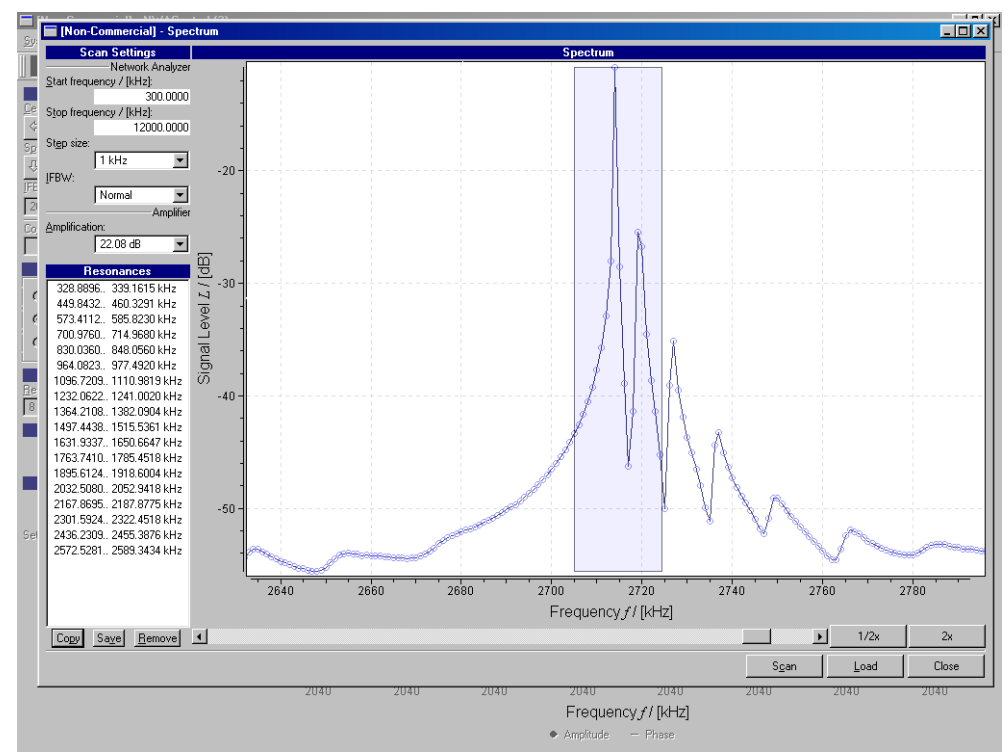

Abbildung B.4: Bildschirmfoto der Resonanzauswahl. Über diesen Dialog kann anhand der Einstellungen in der linken oberen Ecke des Fensters ein Spektrum aufgenommen werden, dessen Resonanzen anschließend einzeln manuell ausgewählt werden. Die Grenzen des blau markierten Bereichs werden in eine Tabelle übernommen, die anschließend während der Messung eingelesen wird. 


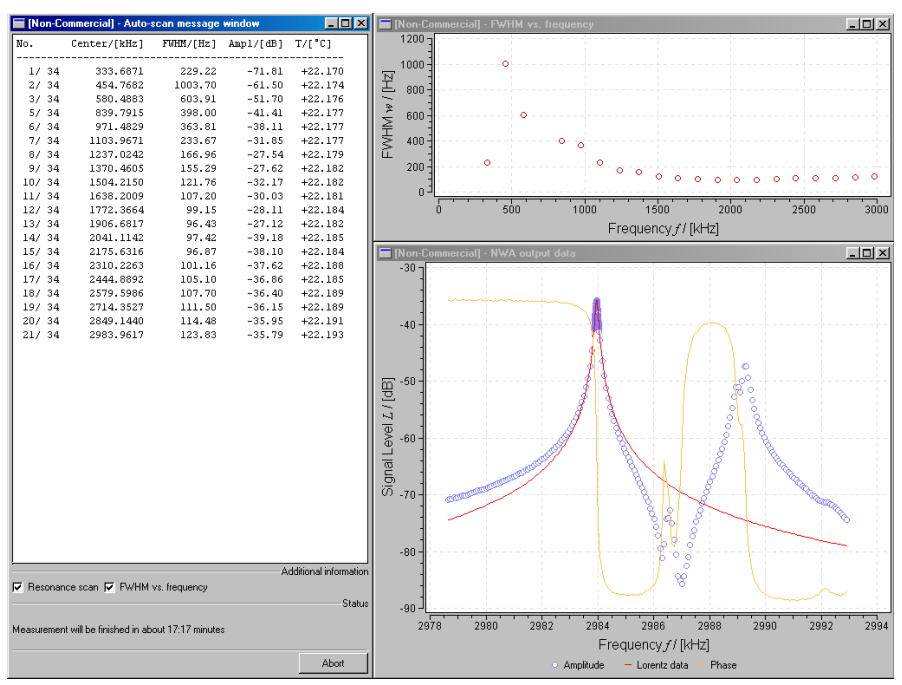

Abbildung B.5: Bildschirmfoto während der Resonanzmessung. Während der Messung der Zellresonanzen werden kontinuierlich die Messwerte graphisch dargestellt. Links befindet sich eine Tabelle mit gemessener Mittenfrequenz, Halbwertsbreite, Amplitude und Temperatur. Auf der rechten Seite ist im oberen Bilddrittel der Verlauf der Halbwertsbreiten über die Frequenz aufgetragen. Aus diesen Daten lässt sich abschätzen, ob während der Messung Probleme auftreten. Rechts unten ist der Resonanz-Scan dargestellt. ( $\circ)$ : Amplitudendaten, (-): Phaseninformation und $(-)$ : Lorentzanpassung. Deutlich ist die hohe Punktdichte in der Hauptresonanz durch den adaptiven Scan-Algorithmus zu erkennen. 
B. Bildschirmfotos von NWAControl 


\section{Velocimetrie-Skript}

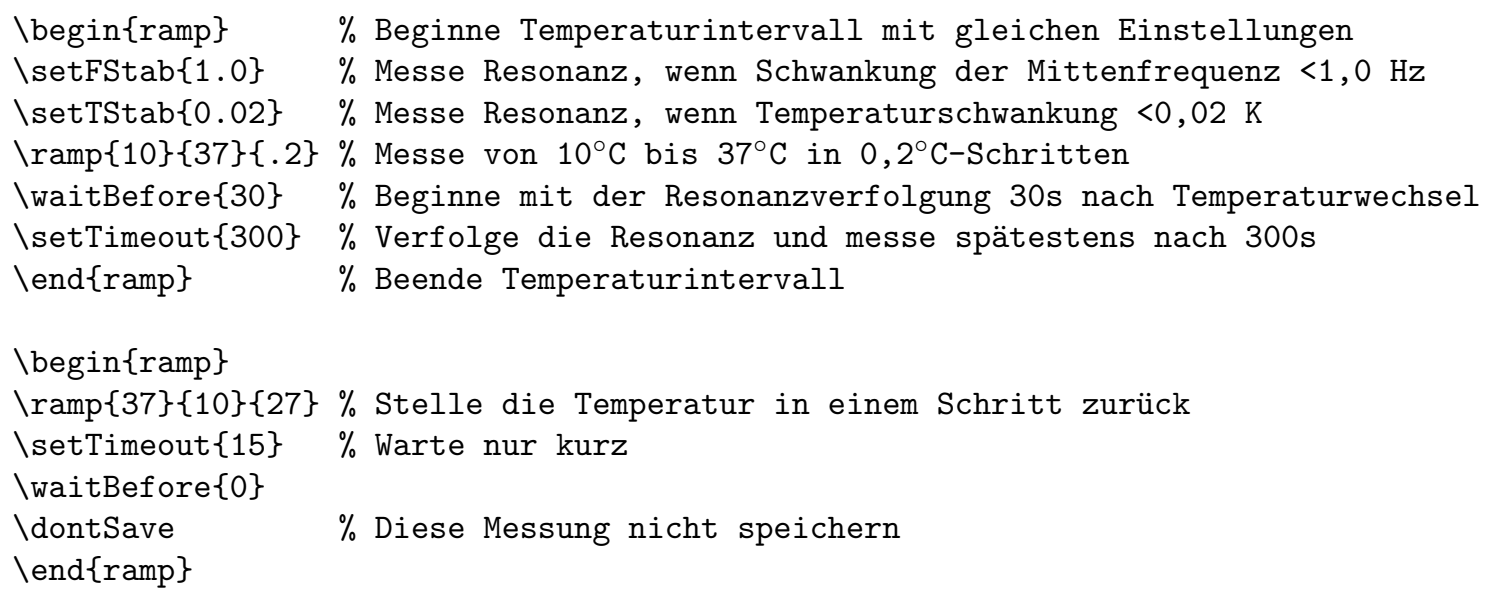

Abbildung C.1: Skript zur Ansteuerung eines Velocimetriemessplatzes. Die Bedeutung der einzelnen Befehle ist nach dem „\%" $\%$-Zeichen angegeben. 


\section{Literaturverzeichnis}

[1] Agilent Technologies: Agilent Spectrum Analysis Basics, Application Note 150; Agilent Tech., Inc. (2005).

[2] Anisimov MA, Pvodyrev AA, Kulikov VD, Sengers JV; Phys. Rev. Lett. 75 (1995) 3146.

[3] Anton Paar: DMA 4500/5000: Betriebsanleitung; Anton-Paar GmbH; Graz (2002).

[4] Avanti Polar Lipids Inc.; www.avantilipids.com; Alabaster, AL (2005).

[5] Baumann G, Müller P; J. Supramol. Struct. 2 (1974) 538.

[6] Behrends R, Halstenberg S, Hoeckel M, Schrader W; nicht publizierte Daten.

[7] Behrends R; Diplomarbeit; Math.-Nat. Fak. Univ. Göttingen (1994).

[8] Behrends R, Kaatze U; J. Phys. Chem. A 104 (2000) 3269.

[9] Behrends R, Iwanowski I, Kosmowska M, Szala A, Kaatze U; J. Chem. Phys. 121 (2004) 5929.

[10] Bergmann L: Der Ultraschall; Hirzel-Verlag; Stuttgart (1954).

[11] Berman HM, Westbrook J, Feng Z, Gilliland G, Bhat TN, Weissig H, Shindyalov IN, Bourne PE; Nucleic Acids Research 28 (2000) 1.

[12] Bernèche S, Nina M, Benoît R; Biophys. J; 75 (1998) 1603.

[13] Berg JM, Tymocz J Stryer L: Biochemistry; 5. Aufl.; W. H. Freeman, New York (2002).

[14] Bhatia AB: Ultrasonic Absorption; Oxford University Press; Oxford (1967).

[15] Bhattacharjee JK, Ferrell RA; Phys. Rev. A 24 (1981) 1643.

[16] Bhattacharjee JK, Ferrell RA; Phys. Rev. A 28 (1983) 2363.

[17] Bhattacharjee JK, Ferrell RA; Phys. Rev. E 56 (1997) 5549.

[18] Bilaniuk N, Wong GSK; J. Acoust. Soc. Am. 93 (1993) 2306.

[19] Bömmel HE, Dransfeld K; Phys. Rev. Lett. 1 (1958) 234. 
[20] Brüning B; Diplomarbeit; Math.-Nat. Fak. Univ. Göttingen (2004).

[21] Buckingham MJ, Fairbank WM; in Progress in Low Temperature Physics, ed. by C. J. Corter; Vol. III; Amsterdam (1961).

[22] Buckin VA; Biophys. Chem. 29 (1988) 283.

[23] Buckin VA, Kankiya BI, Bulichov NV, Lbedev AV, Gukovsky IY, Churprina VP, Sarvazyan AV, William AR; Nature 3401989.

[24] Buckin VA, Kankiya BI, Kazaryan RL; Biophys. Chem. 34 (1989) 211.

[25] Buckin V, Tran H, Morozov V, Marky LA; J. Am. Chem. Soc. 118 (1996) 7033.

[26] Buckin V, Smyth C; Sem Food Anal. 4 (1999) 113.

[27] Cafiso DS; Annu. Rev. Biophys. Biomol. Struct. 23 (1994) 141.

[28] Chen HM, Clayton AHA, Wang W, Sawyer WH; Eur. J. Biochem. 268 (2001) 1659.

[29] Chen FY, Lee MT, Huang HW; Biophys. J. 84 (2003) 3751.

[30] Eggers F; Acustica 76 (1992) 231.

[31] Eichler HJ; in Bergmann-Schäfer: Lehrbuch der Experimentalphysik; Bd. 3: Optik; Hrsg. H. Niedrig; Walter de Gruyter; Berlin (1993).

[32] Ferrell RA; Phys. Rev. Lett. 20 (1968) 665.

[33] Ferrell, RA, Bhattacharjee JK; Phys. Rev. A 31 (1985) 1788.

[34] Finean JB; Chem. Phys. Lipids; 54 (1990) 147.

[35] Fox RO, Richards FM; Nature 300 (1982) 325.

[36] Gerthsen C, Vogel V: Physik; 17. Aufl.; Springer-Verlag, Berlin (1993).

[37] Gennis RB: Biomembranes, molecular structure and function; Springer, New York, 1989.

[38] Guex N, Schwede T, Diemand A; Swiss PDB Viewer, Version 3.6b3; Glaxo Wellcome Experimental Research; Copyright 1995-1999.

[39] Hagen R; Dissertation; Math.-Nat. Fak. Univ. Göttingen (2003).

[40] Halstenberg S; Diplomarbeit; Math.-Nat. Fak. Univ. Göttingen (1997).

[41] Halstenberg S, Heimburg T, Hianik T, Kaatze U, Krivanek R; Biophys. J. 75 (1998) 264.

[42] Halstenberg S, Schrader W, Das P, Bhattacharjee JK, Kaatze U; J. Chem. Phys. 118 (2003) 5683.

[43] Hanke E; Staatsexamensarbeit; Math.-Nat. Fak. Univ. Göttingen (2001). 
[44] He K, Ludtke SJ, Worcester DL, Huang HW; Biochemistry 34 (1995) 16764.

[45] He K, Ludtke SJ, Worcester DL, Huang HW; Biophys. J. 70 (1996) 2659.

[46] He K, Ludtke SJ, Heller WT, Huang HW; Biophys. J. 71 (1996) 2669.

[47] Heimburg T, Würz U, Marsh D; Biophys. J. 63 (1992) 1369.

[48] Heimburg T, Biltonen RL; Biophys. J. 70 (1996) 84.

[49] Heimburg T; Biochim. Biophys. Acta 1415 (1998) 147.

[50] Heimburg T; Biophys. J. 78 (2000) 1154.

[51] Heller WT, He K, Ludtke SJ, Harroun TA, Huang HW; Biophys. J. 73 (1997) 239.

[52] Herzfeld KE, Rice FO; Phys. Rev. 31 (1928) 691.

[53] Hianik T, Buckin VA, Pixnova B; Gen. Physiol. Biophys. 13 (1994) 493.

[54] Hoeckel, M; Diplomarbeit; Math.-Nat. Fak. Univ. Göttingen (1999).

[55] Hohenberg PC, Halperin BI; Rev. Mod. Phys. 49 (1997) 435.

[56] Hope MJ, Bally, MB, Webb G, Cullis PR; Biochim. Biophys. Acta 812 (1985) 55.

[57] Huang HW, Chen FY, Lee MT; Phys. Rev. Lett. 92198304.

[58] Hung WC, Chen FY, Huang HW; Biochim. Biophys. Acta 1467 (2000) 198.

[59] Isambert H; Phys. Rev. Lett. 80 (1998) 3404.

[60] Janiak M, Small DM, Shipley GG; J. Biolog. Chem. 254 (1979) 6068.

[61] Jäger M, Kaatze U, Kudryashov E, O’Driscoll B, Buckin V; New Capabilities of HighResolution Ultrasonic Spectroscopy - Titration Analysis; Spec. Europe (eingereicht 2005).

[62] Jørgensen K, Mouritsen OG; Thermochimica Acta 328 (1999) 81.

[63] Kaatze U, Kühnel V, Menzel K, Schwerdtfeger S; Meas. Sci. Technol. 4 (1993) 1257.

[64] Kaatze U, Kühnel V, Weiss G; Ultrasonics 34 (1996) 51.

[65] Kaatze U, Lautscham K, Brai M; J. Phys. E: Sci. Instrum. 21 (1998) 98.

[66] Kawasaki K; Phys. Rev. A 1 (1970) 1750.

[67] Kohlrausch F: Praktische Physik: Band 3; Teubner-Verlag; Stuttgart (1996).

[68] Kononenko VS; Sov. Phys. Acoust. 31 (1985) 499.

[69] Kratky O, Leopold H, Stabinger H; Zeitschrift für Angewandte Physik 27 (1969) 273.

[70] Kroll DM, Ruhland JM; Phys. Rev. A 23 (1981) 371. 
[71] Kühnel V; Dissertation; Math.-Nat. Fak. Univ. Göttingen (1995).

[72] Labhardt A; Dissertation; Phil.-Nat. Fak. Univ. Basel (1975).

[73] Labhardt A, Schwarz G; Ber. Bunsenges. Phys. Chem. 80 (1976) 83.

[74] Laplace PS; Ann. Chim. Phys. Tome iii (1816) 238.

[75] Lautscham K, Wente F, Schrader W, Kaatze U; Meas. Sci. Techn. 11 (2000) 1432.

[76] Lee CH, Lin WC, Wang J; Phys. Rev. E 64 (2001) 020901.

[77] Lehninger AL: Prinzipien der Biochemie; 2.Aufl.; Spektrum Akademischer Verlag; Heidelberg (1994).

[78] Lipowsky R, Sackmann E; Structure and Dynamics of Membranes; Elsevier; Amsterdam (1995).

[79] Longo ML, Waring AJ, Gordon LM, Hammer DA; Langmuir 14 (1998) 2385.

[80] MacDonald RC, MacDonald RI, Menco BPM, Takeshita K, Subbarao NK, Hu L; Biochim. Biophys. Acta 1061 (1991) 297.

[81] Mayer LD, Hope MJ, Cullis PR; Biochim. Biophys. Acta 858 (1986) 161.

[82] Menzel K; Dissertation; Math.-Nat. Fak. Univ. Göttingen (1993).

[83] Meyer E, Neumann EG: Physikalische und technische Akustik; Vieweg; Braunschweig (1979).

[84] Michels B, Fazel N, Cerf R; Eur. Biophys. J. 17 (1989) 187.

[85] Millero FJ, Ward GK, Chetirkin PV; J. Acoust. Soci. Am. 61 (1977) 1492.

[86] Mortimer, CE: Chemie - Basiswissen der Chemie; 5. Aufl.; Georg-Thieme-Verlag; Stuttgart (1987).

[87] Mouritsen OG, Jørgensen K; Chem. Phys. Lipids 73 (1994) 3.

[88] Nolting W: Grundkurs Physik: Statistische Physik; Zimmermann-Neufang; Ulmen (1994).

[89] Oliynyk V; Dissertation; Math.-Nat. Fak. Univ. Göttingen (2005).

[90] Picker P, Trembley E, Jolicœur C; J. Sol. Chem. 3 (1974) 377.

[91] Pippard AB; Philos. Mag. 1 (1956) 483.

[92] Polacek R; Dissertation; Math.-Nat. Fak. Univ. Göttingen (2003).

[93] Polacek R; Kaatze U; Meas. Sci. Tech. 14 (2003) 1068.

[94] Press WH, Flannery BP, Teukolsky SA, Vetterling WT: Numerical Recipes in C; Cambridge University Press; Cambridge (1988). 
[95] Rubio RG, Ortega F; J. Phys.: Condens. Matter 12 (2000) A459.

[96] Rupprecht A; Diplomarbeit; Math.-Nat. Fak. Univ. Göttingen (1994).

[97] Sarvazyan AP; Ann. Rev. Biophy. Biophys. Chem. 20 (1991) 321.

[98] Schrader W; Dissertation; Math.-Nat. Fak. Göttingen (2001).

[99] Schrader W, Ebel H, Grabitz P, Hanke E, Heimburg T, Hoeckel M, Kahle M, Wente F, Kaatze U; J. Phys. Chem. B. 106 (2002) 6581.

[100] Schrader W, Halstenberg S, Behrends R, Kaatze U; J. Phys. Chem. B 107 (2003) 14457.

[101] Sens P, Isambert H; Phys. Rev. Lett. 88 (2002) 128102.

[102] Singer SJ, Nicolson GL; Science 175 (1972) 720.

[103] Smith RL, Oldfield E; Science 225 (1984) 280.

[104] Stanley HE: Introduction to Phase Transitions and Critical Phenomena; Oxford University Press; New York (1971).

[105] Strehlow H: Rapid Reactions in Solutions; VCH Verlagsgesellschaft; Weinheim (1992).

[106] Telgmann T; Diplomarbeit; Math.-Nat. Fak. Univ. Göttingen (1994).

[107] Telgmann T; Dissertation; Math.-Nat. Fak. Univ. Göttingen (1997).

[108] Tieleman PD, Berendsen HJC, Sansom MSP; Biophys. J. 76 (1999) 3186.

[109] Yang L, Harroun TA, Weiss TM, Ding L, Huang HW; Biophys. J. 81 (2001) 1475.

[110] Zemel A, Ben-Shaul A, May S; Biophys. J. 86 (2004) 3607.

[111] Zhang R, Sun W, Tristram-Nagle S, Headrick RL, Suter RM, Nagle JF; Phys. Rev. Lett. 74 (1995) 2832.

[112] Zuckermann MJ, Heimburg T; Biophys. J. 81 (2001) 2458. 


\section{Danksagung}

Herrn Prof. Dr. Dirk Ronneberger danke ich für die Betreuung dieser Arbeit.

Bei Herrn Prof. Dr. Klaus Bärner bedanke ich mich für die Übernahme des Korreferats.

Herrn Dr. Udo Kaatze gilt mein besonderer Dank für die interessante Aufgabenstellung und die Betreuung und ständige Gesprächsbereitschaft, aus der sich viele wertvolle Hinweise, Anregungen und Ratschläge ergaben.

Dank gilt auch Herrn Prof. Dr. Thomas Heimburg der ebenfalls immer für Ratschläge und Anregungen bei biophysikalischen Fragestellungen zur Verfügung stand.

Bei Dr. Ralph Behrends, Dr. Wilfried Schrader, Stefan Halstenberg und Michaela Hoeckel bedanke ich mich für die zur Verfügung gestellten Messdaten. Herrn Behrends danke ich zudem für seine Bereitschaft, mir bei Fragen zur Theorie der dynamischen Skalierungstheorie und zu Problemen der Ultraschallspektroskopie mit Rat und Tat zur Seite zu stehen.

Ein besonderer Dank gilt Breda O'Driscoll und Prof. Dr. Vitaly Buckin von Ultrasonic Scientific Ltd. in Dublin für die hervorragende Möglichkeit, dort Messungen durchzuführen.

Bei allen aktiven und vielen mittlerweile ehemaligen Mitarbeiterinnen und Mitarbeitern der Arbeitsgruppe möchte ich mich für die konstruktiven Diskussionen und die warme und herzliche Arbeitsatmosphäre bedanken. Es war eine schöne Zeit am DPI.

Bei Herrn Dieter Hille und den Mitarbeitern der Feinmechanischen Werkstatt sowie bei Herrn Dr. Karl Lautscham und den Mitarbeitern der Elektronischen Werkstatt bedanke ich mich für ihren unermüdlichen Einsatz bei der Entwicklung, Wartung und Reparatur der Messapparaturen. Insbesondere die Diskussionen mit Herrn Lautscham über Schallgeschwindigkeitsmessungen waren für diese Arbeit sehr Gewinn bringend.

Ulrike Schulz, Julian Haller und Irek Iwanowski sowie Kevin Brinkmann, Sebastian Dreyer und Erik Süske danke ich für das sorgfältige Korrekturlesen des Entwurfs zu dieser Arbeit.

Mein besonderer Dank gilt meinen Eltern für deren Unterstützung während meines gesamten Studiums. Ohne sie wäre die Anfertigung dieser Arbeit nicht möglich gewesen.

Abschließend möchte ich mich bei der Deutschen Forschungsgemeinschaft und insbesondere Prof. Dr. Dr. Detlev Schild, dem Direktor des Graduiertenkollegs Neuronal Signalling and Cellular Biophysics, für die finanzielle Unterstützung bedanken. 


\section{Lebenslauf}

Name:

Geburtsdatum:

Geburtsort:

Staatsangehörigkeit:

Familienstand:

$08 / 1981-07 / 1985$

$08 / 1985-07 / 1987$

08/1987 - 06/1994

$10 / 1994-10 / 1995$

10/1995

05/1998

$10 / 2000-03 / 2001$

$05 / 2001$

08/2001-11/2001

$11 / 2001-09 / 2004$

$10 / 2004-12 / 2004$

01/2005-05/2005

seit $11 / 2001$
Markus Jäger

3. September 1974

Holzminden

deutsch

ledig

Grundschule Bodenwerder

Orientierungsstufe Bodenwerder

Campe-Gymnasium Holzminden (vormals: Gymnasium Wilhelmstr. 13)

Grundwehrdienst als Stabsdienstsoldat in Stadtoldendorf

Immatrikulation an der Georg-August-Universität Göttingen im Fach Physik (Diplom)

Diplomvorprüfung im Fach Physik (Diplom)

Wissenschaftliche Hilfskraft zur Betreuung „Übungen zur Physik I: Mechanik und Wärmelehre"

Diplomprüfung im Fach Physik (Diplom). Das Thema der Diplomarbeit, die am Institut für Röntgenphysik, unter der Leitung von Prof. Dr. G. Schmahl angefertigt wurde, lautete: „Untersuchungen zur Darstellung von Kernproteinen mit Laser-Scan- und Röntgenmikroskopie“.

Wissenschaftlicher Angestellter am Max-Planck-Institut für Biophysikalische Chemie / Abteilung Membranbiophysik

Stipendiat des Graduiertenkollegs GRK 723: „Neuronal Signalling and Cellular Biophysics" gefördert durch die Deutsche Forschungsgemeinschaft

Wissenschaftlicher Angestellter am Institut für Physiologie / Abteilung Molekulare Neurophysiologie

Wissenschaftlicher Angestellter am III. Physikalischen Institut

Dissertation am III. Physikalischen Institut über „Ultraschallspektrometrie zum dynamischen Verhalten von Domänen in peptidhaltigen Membranen" 